\title{
Experiencing Sexual Victimisation in Childhood: Meaning and Impact - the Perspectives of Child Sexual Abusers
}

\author{
Roberts, Susan M.
}

How to cite:

Roberts, Susan M. (2017) Experiencing Sexual Victimisation in Childhood: Meaning and Impact - the Perspectives of Child Sexual Abusers. Doctoral thesis, Swansea University.

http://cronfa.swan.ac.uk/Record/cronfa40839

Use policy:

This item is brought to you by Swansea University. Any person downloading material is agreeing to abide by the terms of the repository licence: copies of full text items may be used or reproduced in any format or medium, without prior permission for personal research or study, educational or non-commercial purposes only. The copyright for any work remains with the original author unless otherwise specified. The full-text must not be sold in any format or medium without the formal permission of the copyright holder. Permission for multiple reproductions should be obtained from the original author.

Authors are personally responsible for adhering to copyright and publisher restrictions when uploading content to the repository.

Please link to the metadata record in the Swansea University repository, Cronfa (link given in the citation reference above.)

http://www.swansea.ac.uk/library/researchsupport/ris-support/ 


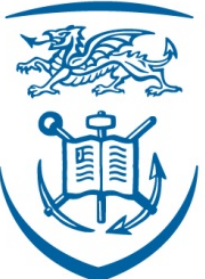

\title{
Swansea University Prifysgol Abertawe
}

\author{
Experiencing Sexual Victimisation in Childhood: \\ Meaning and Impact - the Perspectives \\ of Child Sexual Abusers
}

\section{Susan Roberts}

Submitted to Swansea University in fulfilment

of the requirements for the degree of Doctor of Philosophy 


\section{Summary}

In this small-scale, qualitative, study, the focus is on 40 convicted, imprisoned adult, male child sexual abusers who each reported, during individual interview, that they had been sexually abused in childhood. Thirty two of those men had been abused by males; 5 by females; and 3 by both males and females. Moreover, many had been abused by other children and young people - male and female - some of whom were their siblings; and also by those in positions of trust.

This study is retrospective in design, and grounded in offenders' accounts of the sexual abuse they experienced in childhood; their perceptions of that; its impact on them; and their views as to the extent to which it contributed to their 'becoming an abuser'. The emphasis throughout is on exploring difference within and between groups of offenders. This approach is in direct contrast to the previous tendency to: 'lump all perpetrators together, irrespective of their experiences as a victim’ (Glasser et al. 2001: 483).

Child sexual abusers are more likely to report sexual victimisation in childhood than other offenders and those within the general population. However, there is a dearth of research on their experiences of that abuse and its 'meaning' to them. This research has been undertaken to give voice to a population which is rarely heard; and subsequently to contribute to more effective safeguarding and intervention with both victims and offenders. The research findings highlight the sense of difference evident both within and between groups of offenders, in terms of their victimisation; and also the abuse they subsequently perpetrated. The thesis concludes with some reflection on the implications of this for policy and practice with victims and offenders; and a 'model' of vulnerability is proposed, based on the men's narratives. 


\section{DECLARATION}

This work has not previously been accepted in substance for any degree and is not being concurrently submitted in candidature for any degree.

Signed

(candidate)

Date

\section{STATEMENT 1}

This thesis is the result of my own investigations, except where otherwise stated. Where correction services have been used, the extent and nature of the correction is clearly marked in a footnote(s).

Other sources are acknowledged by footnotes giving explicit references. A bibliography is appended.

Signed (candidate)

Date

\section{STATEMENT 2}

I hereby give consent for my thesis, if accepted, to be available for photocopying and for inter-library loan, and for the title and summary to be made available to outside organisations.

Signed (candidate)

Date 


\section{CONTENTS}

Page no.

Chapter 1: Introduction

Chapter 2: Child sexual abuse

Part 1 - Placing child sexual abuse in context $\quad 15$

Part 2 - Female-perpetrated child sexual abuse 33

Part 3 - Disclosure and the silencing of victims 45

Chapter 3: The Impact of Child Sexual Abuse on Victims

Part 1 - Impact

Part 2 - From Victim to Victimiser 62

Chapter 4: Methodology

Part 1 - Doing research with child sexual abusers: the process $\quad 74$

Part 2 - Doing research with child sexual abusers: the challenges 97

$\begin{array}{ll}\text { Chapter 5: Theoretical underpinnings to the research } & 109\end{array}$

Chapter 6: Research Findings - Male-perpetrated child sexual abuse:

offenders' experiences and perceptions 142

$\begin{array}{ll}\text { Part } 1 \text { - Offenders' experiences of male-perpetrated child sexual abuse } & 148\end{array}$

Part 2 - Offenders' perceptions of male-perpetrated child sexual abuse 188

Chapter 7: Research Findings - Experiencing male-perpetrated

child sexual abuse

Becoming an abuser

Chapter 8: Research Findings - Experiencing female-perpetrated

child sexual abuse

Part 1 - Offenders' experiences and perceptions of female-perpetrated

child sexual abuse

$\begin{array}{ll}\text { Part } 2 \text {-Becoming an abuser } & 264\end{array}$

Chapter 9: Research Findings - Experiencing male and female-perpetrated

child sexual abuse

Part 1 - Offenders' experiences and perceptions of male and

female-perpetrated child sexual abuse

$\begin{array}{ll}\text { Part } 2 \text {-Becoming an abuser } & 290\end{array}$

$\begin{array}{ll}\text { Chapter 10: Discussion and Conclusion } & 297\end{array}$

Discussion - Part 1: Exploring difference 300

Discussion - Part 2: Developing a 'Model' of Vulnerability 313

Discussion - Part 3: The implications of the research 324

$\begin{array}{ll}\text { Conclusion } & 326\end{array}$ 
Page no.

Appendix:

Appendix A - Covering letter

Appendix B - List of questions asked at interview

Appendix C - Consent form for taped interview

Bibliography

335 


\section{Acknowledgements}

I would like to thank my supervisors, Professor Tracey Sagar and Professor

Peter Raynor for their support, guidance and encouragement during the course of the thesis.

I would also like to express my gratitude to Emeritus Professor Maurice Vanstone, and the late Professor Matthew Colton: I am indebted to you both. 


\section{LIST OF TABLES AND FIGURES}

\section{Offenders abused by males:}

Table 6.1: Demographic characteristics

Table 6.2: Offending behaviour

Table 6.3: Victims + duration of abuse

Table 6.4: Offenders’ relationship to victim/s

Table 6.5: Offenders' childhood sexual victimisation - relationship to perpetrator

Table 6.6: Abused by other children and young people

Table 6.7: Abused by those in a position of trust

Table 6.8: Experiencing re-victimisation in childhood

Table 6.9: Offenders' perceptions of intra-familial abuse + details of their subsequent offending

Table 6.10: Offenders' perceptions of extra-familial abuse + details of their subsequent offending

Table 6.11: Offenders' perceptions of extra-familial abuse + details of their subsequent offending

Table 6.12: Offenders' perceptions of extra-familial abuse (in care) + details of their subsequent offending

Table 7.1: Age sexually attracted to children?

Table 7.2: Core ITs evident in narrative?

Offenders abused by females:

Table 8.1: Demographic characteristics

Table 8.2: Offending behaviour

Table 8.3: Victims + duration of abuse

Table 8.4: Offence/s \& offenders’ relationship to victim/s

Table 8.5: Offenders' childhood sexual victimisation - relationship to perpetrator

Table 8.6: Categorizing abuse 
Table 8.7: Offenders' perceptions of their childhood sexual victimisation + details of their subsequent offending

Table 8.8: Core ITs evident in narrative?

\section{Offenders abused by males and females:}

Table 9.1: Demographic characteristics

Table 9.2: Offending behaviour

Table 9.3: Victims + duration of abuse

Table 9.4: Offence/s \& offenders’ relationship to victim/s

Table 9.5: Offenders’ childhood sexual victimisation - relationship to perpetrator

Table 9.6: Categorizing abuse

Table 9.6: Offenders' perceptions of their childhood sexual victimisation + details of their subsequent offending

Table 9.8: Core ITs evident in narrative?

\section{Figures}

Figure 10.1: Factors associated with vulnerability to become a victim of CSA, as evident in a sample of 40 adult male prisoners convicted of child sexual abuse

Figure 10.2: Factors associated with becoming a perpetrator of CSA, as evident in a sample of 40 adult male prisoners convicted of child sexual abuse 


\section{LIST OF ABBREVIATIONS}

CCE: Children's Commissioner for England

CEOP: Child Exploitation \& Online Protection Centre

CJS: $\quad$ Criminal Justice System

CSA: Child sexual abuse

CRB: $\quad$ Criminal Records Bureau

CSEW: $\quad$ Crime Survey for England and Wales

DBS: $\quad$ Disclosure and Barring Service

FSO: $\quad$ Female sexual offending

FSOs: $\quad$ Female sexual offenders

FOI: $\quad$ Freedom of Information (Request)

IIOC: $\quad$ Indecent images of children

ITs: Implicit Theories

MSO: $\quad$ Male sexual offending

MSOs: $\quad$ Male sexual offenders

NSPCC: $\quad$ National Society for the Prevention of Cruelty to Children

ONS: $\quad$ Office for National Statistics

SAB: $\quad$ Sexually abusive behaviour

SHB: $\quad$ Sexually harmful behaviour

SOTP: $\quad$ Sexual Offender Treatment Programme

WHO: $\quad$ World Health Organisation 


\section{Chapter 1: Introduction}

Child sexual abuse (CSA) is a: 'global phenomenon affecting the lives of millions of children' (Stoltenborgh et al. 2011: 90); and a crime which has the potential to devastate victims' lives (Saied-Tessier 2014). However, considerable challenges present in understanding and responding to this problem. In most cases, CSA is not reported, detected or prosecuted (Radford et al. 2011). Indeed, it is a crime that can remain wellhidden, known only to the abuser and victim, because so few victims disclose that they have been abused (Smallbone and Wortley 2001).

Much of the literature on sexual offending focuses on the victimisation of females (O'Leary 2009). Indeed: 'childhood sexual abuse and the sexual abuse of females are typically viewed synonymously' (Spiegel 2003: 11). Scant attention has been paid to males as victims (Easton 2013). As a result, they are a 'marginalized' population (ibid.: 344); and the sexual abuse they experience is often misunderstood, trivialised and denied (Spiegel 2003). The reluctance to focus on male victims and, indeed, even to identify this population, is attributed to a number of factors including: an emphasis on the male perpetrator/female victim model in research, prevention and treatment; a 'social aversion' to the concept that males might be vulnerable to CSA; social constructions of males as aggressive and females as passive; ingrained beliefs that males are self-reliant, even in the face of more powerful abusers; the 'social stigma' associated with homosexuality; the assumptions that female-perpetrated abuse is rare; and social constructions around young males’ sexual activity with older females as a positive ‘encounter’ (Spiegel 2003: 12).

In this small-scale, qualitative, descriptive study, the emphasis is on the voices of those who consistently report sexual victimisation in childhood but whose accounts of that are even less likely to be heard than those in the general male population: prisoners convicted of CSA. Here, their experiences of CSA; their perceptions of that, and its impact on them; and the extent to which, in their view, it played a part in their becoming an abuser are explored. As Plummer (1995: 118) notes, this is a population whose 'own story in their own voice is not heard', in part, because 'nobody will allow it to be told and nobody 
wishes to hear'. Giving sexual offenders a 'voice' requires the challenging of traditional assumptions that they are: 'are at best unreliable in relation to their self-report and are often manipulative, devious and oppressive in their dealings with others' (Hackett and Masson 2006: 184). As a consequence, in a professional sense, the emphasis has been upon the removal of power from those who sexually offend; and the introduction of control mechanisms as a means of reducing future risk. Thus, work with this population, is undertaken amidst a societal background of: ‘intolerance, anger and fear' (ibid.: 184).

When 'safely removed', however, sexual offenders 'have often been the object of great curiousity’ (Lanyon 1991: 35) Indeed, much research has focused on the characteristics of this population; their offending behaviour; the risk they present; and the 'sexually abused-sexual abuser hypothesis’ (Jespersen et al. 2009: 179). Scant attention, however, has been previously paid to the views of offenders' themselves on their childhood sexual victimization (Garrett 2010), particularly where the perpetrator was female. While some would argue against listening to the voices of offenders on their own victimisation, Craissati et al. (2002: 236) assert that there are good reasons to do so, for: 'unless the offender is heard as a victim in his own right, his capacity to develop appropriate victim empathy for his offence victims will be impaired'.

Those who have explored the views of this population have focused on areas such as: prevention (Conte et al. 1989; Elliott et al. 1995); incest and its meaning for fathers who abused their daughters (this research also included daughters’ perspectives on the abuse) (Phelan 1995); the 'thinking and motivation behind abusive behaviour', as demonstrated by those who abused from a position of trust (Colton and Vanstone 1996: 1); treatment (Day 1999; Colton et al. 2009); and treatment and management issues (Hudson 2003; 2005). Moreover, some have examined the views of children and young people who engage in sexually abusive behavior (SAB) on: what they and their parents want from professionals (Hackett et al. 2006); and issues relating to treatment (Lawson 2003). One study (Garrett 2010: 681) explored the childhood experiences of a small number (8) of incarcerated male child sexual abusers. While the focus was on childhood experiences generally as opposed to experiences of CSA, Garrett's work is of relevance to some of 
the men in this sample, whose experience of a 'home space' was characterised by abuse, punishment, violence, rejection, abandonment and a failure to protect.

The research reported in thesis was undertaken in a prison establishment in the UK. Participants included 40 adult, male prisoners convicted of CSA: each reported, during individual semi-structured interviews, that they had been sexually abused in childhood. Thirty two of those 40 men had been abused by males and 8 by females; 3 of the latter had been abused by both males and females. Their stories are told during the course of this thesis. As already noted, such accounts prove unpalatable to some, who experience discomfort and internal conflict at the notion that an offender might have previously occupied the status of victim. The two roles simply do not compute: how can one empathise with a victim who perpetrates sexual violence? To listen to them would surely mean that rather than finding their actions abhorrent, one condones abuse, and decries its impact. Yet, as Hackett and Masson (2006: 184) note, listening to those who have sexually abused: 'is not the same as accepting or colluding with sexual abuse'. For, sexual offenders themselves, many of whom are victims as well as abusers, can provide data which may enable more effective prevention and intervention with both victims and offenders (Elliott et al. 1995).

The emphasis here is on 'self-perceptions' relating to childhood sexual victimisation; and the 'meanings emanating from the abuse dynamics and effects' (Spiegel 2003: 65). The approach adopted throughout this thesis is one of exploring difference between groups of offenders. This approach is taken in direct contrast to the tendency to: 'lump all perpetrators together, irrespective of their experiences as a victim' (Glasser et al. 2001: 483); and to deal with them as one homogeneous mass. Given that the latter has the capacity to mask patterns and characteristics which might aid understanding, a sense of difference is consistently teased out both within and between offender groupings.

Before the aims and objectives of the research are presented, and the research questions listed, the background to the research reported here is set out, in particular, its position as ‘a study within a study’ (Williams 1995: 4). 


\section{Background to the study}

This research was undertaken as part of a wider study which was partially funded by the National Society for the Prevention of Cruelty to Children (NSPCC), and which examined the motivations and strategies of 101 adult, male prisoners who had been convicted of CSA. I was part of the research team involved in that study - which included the late Professor Matthew Colton and Emeritus Professor Maurice Vanstone - and I undertook all 101 interviews. This research focuses on one aspect of that wider study: the accounts of 40 of those 101 men who reported at interview that they had been sexually abused in childhood.

It is important to note at this point, that several peer-reviewed publications have already emerged from the wider study, for example, in relation to: offenders' views on treatment (Colton et al. 2009); sexual abuse by those who work with children (Colton et al. 2010; 2012); and sexual abuse by educators (Roberts and Vanstone 2014). However, the issues reported on here have not previously been published. I have presented some aspects of the work at conference: for example, male child sexual abusers' experiences of femaleperpetrated abuse at the Welsh Centre for Crime and Social Justice (WCCSJ) Conference; and the challenges encountered in undertaking research with child sexual abusers at an NSPCC conference for $\mathrm{PhD}$ students. Moreover, that conference presentation resulted in a publication in a Journal Special Issue (Child Abuse Review) focusing on the methodological issues encountered in doing research with sexual offenders (see Roberts 2011). Aside from this, the work remains 'untouched' and the data are presented here, in their entirety, for the first time.

The decision to pursue a $\mathrm{PhD}$ on this topic was made against a background of interest in one specific aspect of sexual offending: the links between victimization and offending. It was apparent that much attention had been paid to the 'sexually abused-sexual abuser hypothesis' and the proposition that: 'individuals who experience sexual abuse are significantly more likely to later engage in sexual offenses' (Jespersen et al. 2009: 179). It was also clear that such attention often took the form of quantitative research and 
included the use of a range of measures, checklists and tests. Missing from the knowledge base, however, were offenders' accounts of their childhood sexual victimisation. Given this deficiency, and as a means of contributing to the existing evidence, these issues were explored in detail as part of a $\mathrm{PhD}$. I am extremely grateful to the other two members of the research team - Professors Colton and Vanstone - for giving me the opportunity to work with them; to learn from them; and to engage in this research.

At the outset of the wider study, a semi-structured questionnaire was designed to collect as much data as possible from offenders. Those data are drawn on in this thesis as a means of placing the men's stories in context. Section 3 of the questionnaire relates specifically to my research and is entitled: 'The link between childhood and offences'. (A list of the questions asked is included in the Appendix). Further discussion around the questions asked of the men during this part of the interview is included in the Methodology.

Having made clear the background to this research, and its 'place' vis-a-vis the wider study, next, the aims and objectives of this research are outlined and the research questions listed.

\section{Aims and objectives}

The aims and objectives of the research were as follows:

1.To review the literature on the links between childhood sexual victimization and subsequent sexual offending and elicit gaps in understanding and knowledge.

2. To explore child sexual abusers' accounts of their own childhood sexual victimisation.

3. To explore their perceptions of that.

4. To explore their views on the impact of the abuse they experienced. 
5. To explore their views on the extent to which their childhood sexual victimisation played a contributory part in their 'becoming an abuser'.

\section{Research questions}

The following research questions provided direction for the study:

1.What are imprisoned, adult male child sexual abusers' experiences of sexual abuse in childhood?

(a)To what extent, if any, do the experiences of those abused differ according to the gender of the perpetrator? That is, to what extent do the experiences of those abused by males differ from those abused by females; and those abused by males and females?

(b) To what extent, if any, do the experiences of those abused on an intra-familial level differ from those abused by extra-familial offenders?

(c) To what extent, if any, do the experiences of those abused differ according to the age of the perpetrator?

2. What are their perceptions of the abuse they experienced?

(a)To what extent, if any, do the perceptions of those abused differ according to the gender of the perpetrator? That is, to what extent do the perceptions of those abused by males differ from those abused by females; and those abused by males and females?

(b) To what extent, if any, are there differences in perceptions according to the severity of the abuse?

(c) To what extent, if any, do the perceptions of those abused on an intra-familial level differ from those abused by extra-familial offenders? 
(d) To what extent, if any, do the perceptions of those abused differ according to the age of the perpetrator?

3. What, according to offenders, is the impact of the abuse they experienced in childhood?

(a)To what extent, if any, do views on impact differ according to the gender of the perpetrator? That is, to what extent do the views of those abused by males differ from those abused by females; and those abused by males and females?

(b) To what extent, if any, are there differences in views on impact according to the severity of the abuse?

(c) To what extent, if any, do views on impact differ between those abused on an intrafamilial level differ from those abused by extra-familial offenders?

(d) To what extent, if any, do views on impact differ according to the age of the perpetrator?

4. Did offenders disclose the abuse they experienced in childhood?

(a)To what extent, if any, are there differences in disclosure rates according to the gender of the perpetrator? That is, to what extent do disclosure rates differ between those abused by males; those abused by females; and those abused by males and females?

(b) To what extent, if any, are there differences in disclosure rates according to the severity of the abuse?

(c) To what extent, if any, do disclosure rates differ between those abused on an intrafamilial level differ from those abused by extra-familial offenders? 
(d) To what extent, if any, do disclosure rates differ according to the age of the perpetrator?

5. Are offenders' accounts consistent with the existing evidence on the link between childhood sexual victimization and sexual offending?

(a)To what extent, if any, do they see a link between their own childhood sexual victimization and childhood experiences and their subsequent offending?

(b) What, if any, similarities may be found between perpetrators' own experience of CSA and their subsequent offending behavior?

Responses to these questions may be found in the Research Findings Chapters.

\section{The problem of CSA}

Child sexual abuse is a: 'serious and pervasive public health problem, affecting millions of children worldwide' (Brown et al. 2011: 11). It is not a new phenomenon. Rather, sexual violence against children has been perpetrated across time and cultural settings (Smart 2000). In the past few years, however, CSA has consistently featured in the UK media, with high profile cases involving celebrities often dominating news space. The fact that CSA is now discussed more openly in the media has resulted in a positive outcome: victims appear to be more willing to come forward and report that they have been sexually abused (the so-called 'Yewtree effect', resulting from the Jimmy Savile investigation (NSPCC 2013a)). However, as Jutte et al. (2015: 7) note: 'Having the courage to speak out after abuse can be the beginning of a long journey'. Such a journey can be complex and painful, with some victims reporting negative consequences for themselves and their family following disclosure. Moreover, there appears to be little support available to help children recover from abuse if they do disclose (Allnock et al. 2009). 
Of course, what should be acknowledged at this point, is that while high profile cases dominate the headlines, children are actually most at risk from those closest to them: that is, those within their own family or social networks. Indeed, a recent Inquiry into CSA in the family environment by the Children's Commissioner for England (2015: 1) found that approximately two thirds of CSA is perpetrated 'in and around the family'; and in around $25 \%$ of cases, perpetrators are under 18 years of age: namely, brothers or cousins. It is likely that most of the children abused in this way never tell, with evidence suggesting that only 1 in 8 cases of CSA are reported to relevant agencies (ibid.). The issue of disclosure remains an enduring challenge for those agencies who seek to detect abusers and protect children. It is a central feature of this thesis, both because it is a key component in sexual victimisation; and because so many offenders in this sample reported that they had either not disclosed their abuse in childhood, or that they had received an inappropriate response to telling.

With so many children and young people experiencing sexual violence in childhood, attention now shifts, in the final stages of this chapter, to responses to this problem, in legislative and policy terms.

\section{Responses to CSA}

\section{Legislative and policy initiatives}

In the face of increasing concern as to the extent of the problem of CSA, a raft of policy and legislative initiatives have emerged in England and Wales in an attempt to: improve safeguarding; deflect and detect offenders; and manage them through the Criminal Justice System (CJS). It is not intended that an exhaustive account of these are provided here. Rather, some are briefly considered for contextual purposes, including:

Sex Offenders Act (1997) - which established notification arrangements for sexual offenders, with an emphasis on monitoring and reporting. 
Sexual Offences Act (2003) - which included new measures designed to afford increased protection to children and families, for example, in relation to children under the age of 13; and the monitoring of sexual offenders.

Criminal Justice Act (2003) - which included arrangements for assessing the risks posed by violent or sexual offenders; and led to the establishment of Multi-Agency Public Protection Arrangements (MAPPA)

Safeguarding Vulnerable Groups Act (2006) - which established vetting and barring arrangements. The Independent Safeguarding Authority (ISA) was formed to prevent unsuitable adults working with children and to make barring decisions. This body merged with the Criminal Records Bureau (CRB) in 2012, to become the Disclosure and Barring Service (DBS).

Working Together to Safeguard Children (HM Government 2015) - a guide to interagency working to safeguard and promote the welfare of children, with a focus on a child-centred and co-ordinated approach to safeguarding.

Such initiatives speak to a policy agenda of strengthening safeguarding and addressing risk. The Sexual Offences Act (2003), for example, includes:

'a series of measures specifically designed to give better protection to children and families and to put the needs of victims first' (Home Office 2004: 1)

In particular, issues relating to age, gender and the relationship between offenders and victims are addressed (Turton 2008). However, its success will be measured by the extent to which it will result in increased levels of protection for children or in increasing numbers of convictions (ibid.).

In the final section of this Chapter the structure of the thesis is outlined as a means of providing an indication of the intended direction of the work. 


\section{Structure of the thesis}

As already noted, in this thesis, the focus is on 'understanding' child sexual abusers' experiences of sexual victimisation in childhood; their perceptions of that; and its impact on them, in particular, the extent to which it contributed to their becoming an abuser. If we are to make sense of those experiences, it is necessary first to understand the dynamics and effects (Spiegel 2003) of CSA: the factors - both individual and environmental - which render some children and young people more vulnerable to abuse; and some more vulnerable to becoming a perpetrator following abuse; the shape and form of victims' interactions with abusers; and the impact of those interactions. The first task in this process of 'understanding' therefore, and before the men themselves 'speak', involves establishing a base-line and the posing of a number of questions in order to do that: namely, what does CSA 'mean'?; how is it defined and by whom?; what is the extent of the problem?; who experiences such abuse and why?: who perpetrates abuse?; what form does it take?; what is the impact of abuse?; and what is known of the links between victimization and later sexual offending? These questions were instrumental in the initial stages of the literature review process. The content of Chapters 2 and 3, reflect the outcome of that review; and place CSA in context with regard to offenders' childhood sexual victimisation and their later sexual offending.

In Chapter 2, the emphasis first is on the prevalence of CSA, both in the general and offending populations. Then, the characteristics of victims are examined, alongside the factors which might render some children more vulnerable to CSA. This task is undertaken with the intention of shedding some light on the factors which might have rendered offenders in this research vulnerable to experiencing CSA; and which factors might have drawn them to their victims. The characteristics of perpetrators - including female sexual offenders (FSOs) - are examined; and the Chapter concludes with an emphasis on the issue of disclosure of CSA, and the silencing of victims.

In Chapter 3, the focus is on the impact of CSA. The diverse nature of impact and variables associated with more negative consequences of CSA are examined at the outset. 
Then, attention shifts to: the concept of re-victimisation; impact and male victims; the extent to which some victims might report a positive impact; the impact of femaleperpetrated CSA, and positive perceptions of that; and criminal behaviour as a consequence of CSA. Discussion of the latter is then extended to include sexual offending; and issues relating to the pathway from victim to victimiser are examined.

In Chapter 4, the emphasis is on Methodology: on the methods employed in undertaking the research; and the rationale for adopting those methods. The Chapter opens with some reflection on the literature review process, and the development of research questions. The merits of adopting a qualitative as opposed to quantitative approach to the area of enquiry; and the deployment of grounded theory (Glaser and Strauss 1967) techniques are considered. Moreover, access and ethical issues are explored; and the first part of the Chapter concludes with some discussion around data analysis and validity.

In the second part of the Chapter, the focus is on the particular challenges encountered in undertaking research with imprisoned child sexual abusers, with reference to King's (2000: 297-308) 'ten nostrums for field research in prisons'. The concept of emotional literacy, and the 'need to be able to hear accounts of sexually explicit behaviour' (Knight and Modi 2014: 143) is highlighted; and the impact of gender on the interviewing process considered.

In Chapter 5, the focus is on the theoretical underpinnings for the research, in particular, the models/theories which were found to be most relevant, and which informed analysis of specific aspects of the data set. These are listed below:

- Summit's (1983) Child Sexual Abuse Accommodation Syndrome - drawn on in relation to the impact of childhood sexual victimisation; and responses to it

- Spiegel's (2003) Sexual Abuse of Males (SAM) model of dynamics and effects of CSA - drawn on for its specific focus on male victims; and in relation to the impact of childhood sexual victimisation; and victims' response to it 
- Finkelhor and Browne’s (1985) Traumagenic Dynamics Model - drawn on in relation to the impact of CSA

- Finkelhor's (1984) Four Pre-Conditions Model - drawn on in relation to the concept of becoming an abuser: 'the making of a child molester'

- Ward and Keenan’s (1999) Core Implicit Theories - drawn on in relation to the 'thinking' and belief systems which support offending behaviour

Each of these models/theories retain relevance today; and the outcome of their application to the data collected for this study may be found in the Research Findings Chapters. It is also important to note, at this point, that feminist explanations of sexual offending are also of some relevance to the research reported here. They are, however, restricted by the extent to which they can be applied to male victims and female perpetrators. Therefore, while their contribution to the field of sexual offending is acknowledged - and discussed in Chapter 5 - and they are drawn on to some extent during the course of the thesis, their applicability to the data collected is limited.

In Chapters 6, 7, 8 and 9, the research findings relating to the 3 groups of offenders are presented: namely, those men reporting male-perpetrated CSA; female-perpetrated CSA; and male and female-perpetrated CSA. Each of the findings Chapters follows the same structure; and the emphasis throughout, is on the men's accounts and their perceptions of the meaning of their childhood sexual victimisation. Chapter 6 includes the research findings relating to 32 offenders who reported experiencing male-perpetrated CSA. First, their experiences of that abuse are examined. This is followed by presentation of the findings relating to their perceptions of CSA, and its impact on them. Chapter 7 focuses once more on the 32 offenders who reported experiencing male-perpetrated CSA, in particular, on their accounts of 'becoming an abuser'. In Chapter 8, the emphasis is on the 5 offenders who reported experiencing female-perpetrated CSA. First, their experiences of that abuse; their perceptions of it, and its impact on them are explored. Then, attention shifts to their accounts of 'becoming an abuser'. Chapter 9 focuses on the 3 offenders 
who reported experiencing male and female-perpetrated abuse in childhood. Once more, their experiences of that abuse; their perceptions of it, and its impact on them are explored first. Their accounts of 'becoming an abuser' follow.

In Chapter 10 - the Discussion and Conclusion - the research findings are discussed with a particular emphasis on comparing and contrasting findings within and between the 3 groups of offenders. A 'model' of vulnerability is proposed, based on the men's accounts; and on what might be gleaned from those about the factors that might render some children more vulnerable to CSA, and some victims more vulnerable to becoming an abuser. The Chapter concludes with some reflections on the implications of the research for policy and practice with both victims and offenders; and the limitations of the work.

\section{Chapter summary}

In this Chapter, the focus has been on introducing the research reported in this thesis; and making clear its contribution and relevance to existing knowledge on sexual offending against children. The background to the study was outlined; and the study aims and objectives listed. This was followed by the research questions, which were designed to elicit difference within and between groups of offenders. The problem of CSA was then highlighted; and policy and legislative responses briefly considered. The Chapter ended with a focus on the structure of the thesis, as a means of indicating the direction of the work.

In the following two Chapters, the emphasis is on CSA and its impact. 


\section{Chapter 2: Child sexual abuse}

\section{Introduction}

In this Chapter, the problem of CSA is explored. The Chapter is presented in 3 parts. In Part 1 , the focus is on understanding the extent of CSA; and the characteristics of victims and male perpetrators. In Part 2, the emphasis shifts to female-perpetrated CSA; and in Part 3, issues relating to disclosure and the silencing of victims are examined.

As already noted, much of the literature on sexual offending refers to males as perpetrators and females as victims. Females who sexually offend against children are often subsumed in the literature on sexual offending generally. Indeed, there is a: 'presumption of maleness in discussions of sexual abusers' (Finkelhor 1984: 37). Thus, for sexual offender, read male. Such presumptions mask important differences between MSOs and FSOs and the abuse they perpetrate; and thus inhibit understanding. In an attempt to redress the balance somewhat, a decision was made to examine FSO separately, in Part 2 of this Chapter.

\section{Part 1: Placing CSA in context}

Child sexual abuse has been recognised as a 'major social problem' (Smallbone and Wortley 2001: 1). However, there are limitations to the evidence, research and otherwise, on CSA. Thus, only a partial account of the sexual victimisation of children is available. Attempts to understand the problem are compounded by: the hidden nature of sexual offending against children; the fact that there is not one single accepted definition of CSA; that victims - and others - might not define what happened to them as abusive and therefore, this impacts on the task of establishing the extent of abuse; that many victims do not disclose CSA; that data available represent only a fraction of the sexual offences committed; that offenders differ in terms of their characteristics and offending behaviour: this compounds detection; and that the impact of abuse is variable.

In the opening section of this Chapter, the focus is on the difficulty associated with defining CSA. This serves a number of functions: first, an understanding of the conflicting and contradictory nature of definitions is essential to the process of assessing the findings of available research on CSA. Second, an understanding of the complexity associated with 
defining CSA is necessary if the experiences and accounts of the men who participated in this research are to be 'understood'.

\section{The problem of definitions}

Johnson et al. (2006: 76) draw attention to the fact that: 'unfortunately, there is no consistent definition of CSA shared by all researchers'. Indeed, emphasis is placed on a disparate range of factors, including: the use of force; age differentials; sexual gratification; developmental factors; the part played by positions of power, trust and responsibility; and the issue of consent. The challenges encountered include those resulting from the adoption of differing 'parameters' (Browne and Finkelhor 1986: 66); namely: 'differences of definition and methods of study' (Glasser et al. 2001: 483). Moreover, some research focuses on abuse perpetrated by adults and excludes sexually harmful behaviour by children and young people (SHB). Other research focuses solely on one form of abuse, for example, intrafamilial abuse (Browne and Finkelhor 1986).

Differing methods of data collection, for example, interviews or surveys, and the use of a range of definitions, result in varying results which inhibit comparison across studies (Hanson and Slater 1988). Indeed, studies are limited by the fact that they either draw on wide-ranging definitions which include all forms of sexual violence, or on definitions restricted to physical sexual contact (ibid.). These issues have been raised at the outset of this thesis as a means of highlighting their significance to the field of inquiry. They are explored in further detail in the Methodology as part of the discussion as to how the question of sexual victimization in childhood was posed to the men in this sample; and the challenges encountered in that. The focus next, is on 'official' definitions of CSA. This is followed by some reflection on the extent of the problem as it emerges in specific populations: the general population; and the population of most relevance to this thesis: the sexual offending population.

In an 'official' sense, the most recent definition of CSA (applicable in England and introduced post-data collection for this thesis) proposes that CSA:

'Involves forcing or enticing a child or young person to take part in sexual activities, not necessarily involving a high level of violence, whether or not the child is aware of 
what is happening. The activities may involve physical contact, including assault by penetration (for example, rape or oral sex) or non-penetrative acts such as masturbation, kissing, rubbing and touching outside of clothing. They may also include non-contact activities, such as involving children in looking at, or in the production of, sexual images, watching sexual activities, encouraging children to behave in sexually inappropriate ways, or grooming a child in preparation for abuse (including via the internet). Sexual abuse is not solely perpetrated by adult males. Women can also commit acts of sexual abuse, as can other children.' (HM Government 2015)

This definition usefully acknowledges the fact that abuse might not be violent in nature; and that a child might not be aware that what they are experiencing is abuse. It also draws attention to the production of sexual images and Internet offending - both of which are proving increasingly difficult to detect and prosecute. Moreover, there is recognition here that females and children and young people may also be perpetrators. Missing, however, is reference to the concept of informed consent; the violation of laws and also social taboos; and the part played by positions of responsibility, trust and power in the process of abuse (WHO 2006).

\section{The extent of the problem of CSA}

Attempts to understand the extent of the problem of CSA draw on a range of sources, including official statistics. All prove problematic; and this section opens with some discussion around the problem with statistics.

The problem with statistics

Significant numbers of children and young people experience CSA; and in the main, victims are female (Pereda et al. 2009). Yet, data which are available represent only those cases which come to the attention of relevant authorities; are reported; and recorded as crimes. Much sexual offending against children remains hidden, particularly where the victim is male (O’Leary and Barber 2008). A number of factors come into play here, including a cultural reluctance to view males as victims; and males' perceptions of themselves as self-reliant and 
able to prevent abuse - thus disclosure is less likely to occur (Romano and DeLuca 2001; Spiegel 2003).

In 2013-2014, a total of 72,977 (MOJ 2015) sexual offences were recorded in England and Wales. Much of the statistical evidence on sexual offending relates to offences against both adults and children. However, data elicited by the NSPCC (National Society for the Prevention of Cruelty to Children) through a Freedom of Information Request (FOI) request indicate, that in the same period, a total of 36,429 sexual offences against children were recorded in the UK: the highest number of such offences in the last decade. This is likely to be a direct result of the Savile investigation and the so-called 'Yewtree effect' (Jutte et al. 2015); and also improvements in police recording following an investigation by HMIC which highlighted the issue of under-recording of sexual offences (HMIC 2014). In contrast, over the 2 year period 2012-2014, estimates of the 'dark figure' for CSA prevalence indicate that between 400,000 and 450,000 victims experienced CSA in England (CCE 2015: 3).

More recently, another FOI Request from the NSPCC (in March 2016) to all UK police forces has elicited some interesting, if concerning, data. The charity draws attention to the significant increase in reported CSA cases during 2014/2015. A total of 45,456 cases were reported to police forces across the UK in that year; 41,457 of those were in England and Wales (an increase of $32.7 \%$ on the previous year). This increase is attributed to: improvements in police recording; increased awareness on the part of the public resulting from media reporting of CSA; and increased levels of on-line grooming. During 2014/15, five child sexual offences were reported to police every hour: a total of 124 each day. Some 10,757 sexual offences related to children under the age of 10; 2,409 of those victims were very young children who had not yet reached school age. Moreover, there were almost 4 times as many cases involving girls as victims $(32,675)$ than boys $(8,387)$. For those who work with children, including organisations such as the NSPCC (2016), the increase in reported cases is 'deeply worrying'; and indicative of the 'widespread' nature of sexual offending against children.

The most recent recorded crime data in England and Wales (for year ending March 2016), indicate that there were 39,813 sexual offences against children under the age of 16 (ONS 2016). Of note, is that police recording has recently begun to focus on collecting data relating to the age of victims. This is a useful step forward in determining the extent of CSA. Of 
course, it will not provide a definitive account of the problem. Indeed, as already noted, a recent report from the Office of the Children's Commissioner in England (2015), focusing on CSA within the family, suggests that only 1 in 8 cases are brought to the attention of relevant agencies. In light of this, and in a further attempt to determine extent, attention now shifts to what is known of the prevalence of CSA in the general population.

\section{Prevalence of CSA in the general population}

Attempts to understand the extent of the problem of CSA often draw on incidence and prevalence rates. Incidence refers to the number of cases which occur over a specified period of time; and prevalence, to the number of children who experience sexual victimisation, either on one or more than one occasion (Fallon et al. 2010). The former relates to cases which are reported or detected, usually in the context of child protection services; the latter is associated with the self-reporting of abusive experiences.

Evidence as to the extent of the problem of CSA remains contradictory, with prevalence 'notoriously difficult to estimate' (Brown et al. 2011: 10).This is due to the methodological limitations of much of the work in this area. Of course, much abuse is never disclosed and so will not appear in any official account of the problem (Allnock et al. 2009). Moreover, as many studies focus on retrospective accounts from victims, research may be limited by: some victims' decisions not to disclose; poor memory recall; or the reconstruction of events in order to make sense of what happened (Williams 1994). Given this, it is likely that the available prevalence data represent a significant underestimation of the problem (Brown et al. 2011).

Prevalence rates for CSA in the general population vary, according to the research sample; 'the questions asked; and the definition of sexual abuse' (Craissati et al. 2002: 226). In the US, rates have been found to range from 19\% for females to 9\% for males (Finkelhor 1981); and $27 \%$ for females, and $16 \%$ for males (Finkelhor et al. 1990). In the UK, rates of $21 \%$ for females; and 11\% for males have been reported (Cawson et al. 2000). Some interesting variations in rates emerge in a global sense: ranging from $7 \%$ to $36 \%$ for women and from $3 \%$ to $29 \%$ for men (Finkelhor 1994). A more recent meta-analysis by Stoltenborgh et al. (2011) estimated global prevalence rates for females at 18\% and males, 7.6\%. Once more, methodological variations across studies account for such differences, for example, the use of 
random as opposed to convenience samples; and the use of varying instruments, including telephone and also face to face interviews.

In the midst of such divergence, there appears to be one constant: girls appear to be more likely to be sexually victimised than boys (Pereda et al. 2009). Indeed, Smallbone and Wortley (2001) found that females were 3 times more likely to be abused than males; and in cases of intra-familial abuse, females were 10 times more likely to be victimised (ibid.). According to Finkelhor et al. (2005), 1 in 12 children will experience CSA in any given year, with females 6 times more likely to be victims than males.

\section{A new and alternative data source}

Most recently, (August 2016), additional data have emerged in England in Wales on the extent to which adults have experienced CSA (ONS 2016). Data on physical, sexual and psychological abuse were collected for the first time from those aged 16 to 59 years, as part of the Crime Survey for England and Wales (CSEW) 2015-2016. These represent 'the first official statistics on prevalence of abuse during childhood' (ONS 2016: 4). An estimated 567,000 females aged 16 to 59 years reported experiencing sexual assault in childhood; the figure for males was 102,000. Females were 4 times as likely as males to report sexual assault in childhood (11\% as opposed to $3 \%)$. Females were also more likely than males to experience rape or penetration (including attempts): here, rates were $3 \%$ as opposed to $1 \%$.

Clearly, the move to include a focus on self-report of CSA in the most recent CSEW survey is a welcome initiative. However, there are some limitations to the work: in particular, the fact that questions on abusive experiences focused on adult perpetrators only. Sexual assault was defined as rape or penetration (including attempts); and other forms of sexual assault including indecent exposure or indecent touching/kissing of a sexual nature by an adult. While the category 'other family member' is described as including siblings, the extent of SHB by other children and young people is not obvious from the data collected. Given that evidence suggests that much CSA is perpetrated by other children and young people, this account of victims' experiences is therefore, once more, partial.

It appears that a preoccupation with numbers, takes us no further forward in dealing with the problem of CSA. Examination of the research evidence on prevalence, does highlight a 
number of issues, however: namely, the complexity associated with undertaking research on CSA; the difficulties inherent in drawing comparisons between studies; and the fact that wherever studies have been undertaken - and although rates are likely to be an underestimate - many children and young people report experiencing CSA. Of course, the challenge lies in addressing that; and protecting those who are vulnerable from CSA.

\section{Prevalence of CSA in sexual offending populations}

Glasser et al. (2001: 482) propose that given the contradictory nature of research: 'a cautious estimate of sexual victimisation in the male general population would be about $10 \%$.' The extent to which this estimate is applicable to the sexual offending population is explored next.

It appears that male child sexual abusers are more likely to report a history of CSA than males in the general population, and other offenders (Johnson et al. 2006). Indeed, evidence suggests that significant numbers of sexual offenders have themselves experienced CSA (Dhawan and Marshall 1996). Researchers focusing on incarcerated males have found rates ranging from 14.3\% (Weeks and Widom 1998); to 59\% (Johnson et al. 2006); and 75\% (Romano and De Luca 1997). Others, who have focused on sexual offenders in treatment, report rates of 46\% (Craissati et al. 2002); and 42\% ( Langevin et al. 1989). Yet others, have engaged in comparative studies, comparing the CSA histories of child sexual abusers and rapists. Of note, is the finding from much of this research, that child sexual abusers are more likely to report CSA than rapists (Lee et al. 2002; Simons et al. 2002; 2008). Of interest, however, is that in Connolly and Woollons' study (2008: 119), a higher percentage of rapists (71\%) reported CSA than child sexual abusers (45\%). This finding is not consistent with other research referred to above.

Evidence of childhood sexual victimisation is consistently found in populations of adult sex offenders and also in another population of relevance to this thesis: children and young people who engage in SHB (Hackett et al. 2013). Harmful sexual behaviour is defined as sexual behaviour between children, which is characterised by coercion or a threat of violence (NSPCC 2013). It can include sexually explicit discussion, or acts which are age or developmentally inappropriate, including penetrative sexual activity (Rich 2011). In the UK, between one fifth and one third of CSA is perpetrated by children and young people (Hackett 2004). There is a growing body of evidence on the latter, and in recent years, researchers 
have explored a range of issues including: the impact on practitioners of working with these children and young people (Almond 2014); their successful transition back into the community (Lambie and Price 2015); risk assessment (Yates et al. 2012); and assessment and treatment issues (Worling 2012). Moreover, some have focused on practice and policy developments and their effectiveness (Smith et al. 2014); on the evaluation of treatment programmes (Edwards et al. 2012); and on the outcomes of interagency training for those who work with these children and young people (Hackett et al. 2012).

Research on the backgrounds of these young people is contradictory, (Finkelhor et al. 2009); and data limited, in the sense that they have usually been drawn from small samples in one geographical location or site (Hackett et al. 2013). They are a 'complex' (Smith et al. 2014: 277) and heterogeneous population (Edwards et al. 2012; Hackett et al. 2013; Vizard et al. 2007): the majority are adolescent males whose backgrounds are often characterised by histories of sexual, physical and/or emotional abuse (ibid.). Some have mental health issues, and exhibit conduct disorder, and post-traumatic stress disorder (PTSD) (Epps and Fisher 2004). In addition, they tend to present with anxiety; low self-esteem, and social and interpersonal skills; and lack sexual knowledge (Hackett 2014). Such factors can lead to problems in developing relationships, and subsequently to SHB (NSPCC 2013c).

The majority of their victims are family members (Yates et al. 2012), and younger children (Hackett et al. 2013); and the abuse they perpetrate is thought to be a response to their own experience and an attempt to regain control (Watkins and Bentovim 1992). Some research evidence points to rates of CSA in this population of around 40\% (Cooper et al. 1996; Worling 1995); this is considerably higher than the $10 \%$ rate for the general male population (Watkins and Bentovim 1992). Others cite rates ranging from 19\% to 55\% (Worling 1995); 31\% (Hackett et al. 2013); and 71\% (Vizard et. al. 2007).

Having established the extent of the problem of CSA to a certain degree, attention now shifts to the characteristics of those who experience CSA.

\section{Victims}

The actual number of CSA victims is not known (Saied-Tessier 2014); and the divergence in evidence on this has already been highlighted. It appears that, where there is physical 
violence in the family, it is more likely that CSA is also perpetrated (Cawson 2000). Evidence relating to the age of victims is often conflicting. Howitt (1995) and Finkelhor (1986), suggest that the peak age of victimisation is 7 to 12 years; and between 8 and 12 years respectively. Others (HM Government 2007), suggest that CSA is most prevalent in the age range 5-14yrs. Some posit that boys are more likely to be older than girls at the point of victimisation (see, for example, Finkelhor 1979; 1984; Baker and Duncan 1985; Russell 1989b). Others, however, have found male victims to be younger than females (Fischer and McDonald 1998) when first abused. It appears that older boys are less likely to be abused than older girls. Perhaps of relevance here, is that offenders do not find older boys as 'desirable sexually' as pre-pubescent boys (ibid.: 926). Such contradictions may arise once more, from the fact that: data sources differ, with some research reliant on self-reports of victims disclosing their age at onset of abuse; other research relies on medical records or other case records which indicate the age at which abuse is detected by professionals (Plummer and Cossins 2016; Finkelhor 1984). Moreover, one cannot discount the gender, and other, differences evident in terms of disclosing abuse.

Much of the research on victimisation has focused on females or has included small samples of males (Finkelhor 1984). Mendel (1995: 1) observes that: 'Male survivors of child sexual abuse constitute an extremely under-identified, underserved, and, all too often, misunderstood population.' This, is attributed to 'societal myths' which influence understanding of the meaning of male and female: in particular, the sense in which males have the strength and capacity to protect themselves from harm; and are more likely to victimise others than become victims themselves. Mendel also draws attention to another factor which influences under-reporting and under-recognition of male victimisation, that is, the belief that males' sexual appetite renders them 'willing and eager' to engage in sexual activity; the corollary of this, is that any form of such activity is not abusive, but rather, is 'welcomed' by the male (ibid.: 18).

Some research indicates that boys are: most likely to be abused by males (McGuffey 2008); more likely to be abused by a non-family member (Maikovich-Fong and Jaffee 2010; Ogloff et al. 2012); and 'in conjunction with other children'; are more likely to live in poverty as part of single-parent households; and to also experience physical abuse (Finkelhor 1984). To this, Spiegel (2003) adds that boys are more likely to experience: abuse which is more severe in nature; physical violence in childhood, either as part of their sexual abuse or external to that; 
exposure to pornography; abuse characterised by coercion; penetrative sexual activity - with a penis or object; genital mutilation or violence; to be forced to perform fellatio or cunnilingus on their abuser; or to be coerced into penetrating their abuser with either their penis or an object. Plummer and Cossins (2016: 14) draw attention to the diverse nature of CSA experienced by boys, when compared with girls. They suggest - from their review of the literature - that boys are less likely to experience CSA which is persistent or protracted; are more likely to be abused by family members of a similar age, their peers, professional perpetrators, females or strangers; are more likely to experience 'less serious' abuse; and to have positive or mixed perceptions of their abuse, regardless of the gender of their abuser.

Moreover, others suggest that in terms of public perception, male victims are more likely than females to be deemed responsible for their own abuse; to be viewed negatively if they disclose; and to be regarded as: 'gay, deviant, and a potential perpetrator' (Spiegel 2003: 100). Males are less likely to perceive the sexual activity with their perpetrator as abuse; to disclose it; to be protected by their family if they do disclose; or encounter 'parental concern' about the impact of abuse, aside from anxieties about sexual orientation (ibid.). Given the latter, it appears that, as Mendel (1995: 20) notes: 'it is easier to recognise a male perpetrator than a male victim'.

\section{Vulnerability}

All children are vulnerable to abuse, by virtue of their status and position in society. Children's powerlessness is demonstrated on a number of levels: namely, as a result of their immaturity; lack of physical strength; and knowledge of all things social and sexual (La Fontaine 1990). Their relationships with adults are characterised by inequality and vulnerability: both ensure that victims experience confusion and a sense of guilt at their own victimisation (ibid.). Indeed, it appears that victims are easily convinced they are to blame for the sexual abuse they experience, and this works to abusers' advantage (Roberts and Vanstone 2014). Such powerlessness is effective in controlling and silencing victims (Turton 2013), and warrants some attention. For, as Kitzinger (1988: 83) observes: 'If we are to tackle the roots of child sexual abuse we have to think about the position of children in society'.

Some children are even more vulnerable to CSA than others, for example, children with disabilities (Brown et al. 2016; Miller and Brown 2014). Indeed, some (Jones et al. 2012; 
Sullivan and Knutson 2000) have found that children with disabilities are up to 3 times more likely to be abused than children within the general population. A number of factors increase the risk of abuse in the former (NSPCC 2014). These include: their dependence on others for personal care and support; difficulties with communication and understanding, and in particular, the ability to seek help; the isolation often experienced by families with children with disabilities; and societal attitudes about disability which often compound that sense of isolation - these attitudes take the form of a reluctance to acknowledge that children with disabilities are abused; and minimise the impact of such abuse (ibid.).

Children with behaviour or conduct disorders; those with hearing impairments; difficulties with speech and language; and health issues, appear to be at greatest risk of abuse (NSPCC 2014; Save the Children 2011). Children who have been placed in local authority care are at particular risk of abuse (Brown et al. 2016), both whilst in the care system and on leaving it (Coy 2008); and children with disabilities who are placed in residential care are at increased risk (Sullivan and Knutson 2000). Also at heightened risk, are children who have previously experienced CSA; are isolated from their peers (Elliott et al. 1995); and live in a household characterised by physical violence (Fleming et al. 1997); domestic violence; and parental substance abuse (Goodyear-Brown 2012).

Other factors of note, include: parental illness or disability, and a lack of 'physical affection' from a father (Finkelhor 1984: 26); the presence within the household of a father who is not biologically related to the child (Finkelhor et al. 1990); poor relationships with caregivers, in particular, lack of affection from mothers; social isolation (Finkelhor 1984); and poor parental attachment (Fleming et al. 1999). On an individual level, the following also feature: diminutive physical stature; low self-esteem; and fearfulness. In an environmental sense, living in a single-parent household, and in an unsafe neighbourhood are also associated with increased vulnerability (Boney-McCoy and Finkelhor 1995). 'Indirect victimization' (where a child is affected by the victimisation experienced by a family member, and feels vulnerable and powerless as a result) also increases the risk of later CSA (ibid.). This is of particular relevance to this thesis as some offenders abused female victims whose mothers - and other family members - had also previously experienced CSA.

While some children are clearly vulnerable to CSA, they are not responsible for their own victimization (Black et al. 2001). Rather, responsibility lies wholly with perpetrators. 
Offenders will not abuse all those children whom they have access to: they are more likely to select victims based on their vulnerability; and are skilled at recognising such vulnerability and capitalising on it (Roberts and Vanstone 2014). The extent to which offenders in this sample demonstrated vulnerability; and the traits which proved 'attractive' in their victims are considered in the Research Findings Chapters. The emphasis shifts now to those who perpetrate CSA.

\section{Perpetrators}

Child sexual abusers are a heterogeneous population. They have 'unique personal and criminal histories' (Robertiello and Terry 2007: 509); and demonstrate variations in the attitudes and beliefs which support their deviant behaviour. Evidence suggests that they do, however, share some characteristics: for example, poor social skills; low self-esteem; feelings of inadequacy; loneliness; worthlessness; a sense of vulnerability; and in some cases, an inability to develop and maintain appropriate relationships with others (ibid.). Most abusers are adults, usually aged between 20 and 60 years (Fondacaro et al. 1999). Those who abuse males are usually between 11 and 20 years older than them (ibid.). Most abuse is not perpetrated once, as an isolated incident (Feiring et al. 1999). The majority of offenders abuse one victim at a time; however, in some cases they may offend against several victims at the same time, both on an individual and group level; and many report that stress is often a precursor to offending, for example, work related stress; and difficulties with sexual relationships (Elliott et al. 1995). These issues are explored in further detail during the course of the thesis. This section begins with some reflection on the 'male monopoly' in the commission of CSA.

\section{The 'male monopoly'}

There appears to be a 'male monopoly' (Freel 2003: 482) in the perpetration of CSA; and the language used in the literature on sexual offending reinforces this, with perpetrators consistently referred to as 'men'; 'males'; and 'he'. Evidence suggests that males perpetrate between $90 \%-98 \%$ of sexual abuse against girls, and $80 \%-86 \%$ of sexual abuse against boys (Finkelhor 1990; Finkelhor et al. 1986). The fact that CSA is 'committed primarily by men' (Finkelhor 1984: 11) also appears to be supported by data available on the sexual offender prison population. On $31^{\text {st }}$ December 2015, the prison population in England and Wales stood 
at 84,968 . Some 82,036 of that population were male. At that point, there were 11,975 sentenced sexual offenders in prison in England and Wales - this was a 9\% increase on the previous 12 months. Of those, once more, the vast majority $(11,866)$ were male. Some 11,583 were adult males; 248 were aged 18-20 years; and 35 were aged 15-17years. In contrast, there were only 109 females in prison for sexual offending; 106 of those were adults and 3 were aged 18-20 years (MOJ 2016). As is so often the case with data on sexual offending, it is not clear how many of these offenders had sexually abused children.

\section{The Paedophile}

In any discussion of sexual offending against children - in a popular or media context -, the issue of the paedophile often predominates; and the perception of the sexual offender as distinctly different to others and out of the ordinary, persists. With children regarded as: 'the quintessential innocent victims', those who sexually abuse them are viewed with contempt (Miller 2013: 506); and perceived as existing outside the bounds of normality. The media have a 'profound' (Weatherred 2015: 16) influence on public perception and understanding of CSA; and are the means by which most gain knowledge of the problem (Kitzinger 2004). Yet, media representations can be misleading as they often focus on the most sensational but rare - cases (ibid.), in which perpetrators are unknown males (Turton 2013). These include cases of child abduction by strangers (Weatherred 2015). Intra-familial CSA either remains 'absent from media reporting' or is 'spectacularised' in a way which makes it appear abnormal (Turton 2013: 78).

In the UK, the tragic case of Sarah Payne is a useful illustration of the media's preoccupation with the 'stranger danger' element of sexual offending (BBC News 2001; The Telegraph 2001). Such offenders are often perceived as almost demonic in shape and form and thus easily recognisable. The images of Roy Whiting - the sex offender who murdered Sarah present in numerous media sources serve to reinforce misconceptions of child sexual abusers and paedophiles as representative of all things evil and every parent's nightmare; and 'as something other and not like us' (Turton 2008: 6). Unfortunately, until such stereotypical images - and misunderstandings - are addressed, children will remain vulnerable. For, they are most at risk from those closest to them (ibid.). 
The terms paedophile and paedophilia serve to deflect attention away from: 'recognition of abusers as “ordinary men” - fathers, brothers, uncles, colleagues'; and also from the part played by power and control in sexual offending against children (Itzin 2000: 90). Paedophilia is viewed - according to psychiatric classification - as: 'one of the sexual paraphilias alongside fetishes and sexual activity with animals' (Howitt 1995: 14). Those who sexually abuse children are often referred to by the all-encompassing term paedophile, even though the APA (2000) definition specifies that paedophilic sexual activity involves pre-pubescent children. Indeed, paedophilia is defined as:

'recurrent and intense sexual urges and fantasies involving pre-pubescent childrelated sexual activity of at least six months duration, that have been acted upon or cause significant distress to the person experiencing them'. (DSM IV-TR American Psychological Association, APA, 2000)

To clarify then, paedophilia refers to a sexual interest in children - who have the body shape of a child under 11 years of age - which persists over time (Freund et al. 1984). The term used to describe those who are sexually aroused by children who are post-pubertal - that is, between the age of 11 and 14 years (Blanchard et al. 2009) - is hebephilia (Wyre 2000: 49). This term, however, has not: 'become widely used' (Blanchard et al. 2009: 335) even by those who work in a professional sense with sexual offenders. A third term - ephebophilia relates to those who have a sexual interest in children in the age range mid-to-late adolescence (Miller 2013). In spite of these distinctions in terms of age preference, however, paedophilia appears to have become an accepted label for child molestation, whatever the age of the victim/s. Of course, as noted above, these terms are psychiatric classifications - a crime is committed only if the individual moves beyond the world of fantasy to commit a sexual offence (Miller 2013).

The term paedophile is not used during the course of this thesis to refer to offenders in this sample (unless, of course, the men, in speaking of their offending, refer to themselves as such). Given that a psychiatric assessment did not form part of the research, the men are referred to as prisoners who had been convicted of CSA. The frequently used term in much of the literature on sexual offending against children - child sexual abusers - is also used during the course of the thesis. Notwithstanding such debates about the use of language, some attempts are made in analysing the data collected, to differentiate between offenders in terms 
of their preference as to the age of their victims; and also the extent to which they might be classed as fixated or regressed offenders.

\section{Classifying sexual offenders: fixated or regressed?}

Much effort has been expended in attempting to classify male sexual offenders (MSOs) as a means of understanding their offending behaviour and assessing risk (Wyre 2000). One of the most often cited classifications is that of the fixated or preferential, versus the regressed or situational offender (Bogaerts et al. 2005; Groth and Birnbaum 1978; Miller 2013). Regressed - or non-fixated - offenders' sexual interest in children usually emerges in adulthood. They exhibit an age-appropriate sexual preference, usually heterosexual. However, in stressful times they seek out children as a means of satisfying their emotional needs (Sgroi 1982).

Fixated offenders are defined as those who have: 'from adolescence been sexually attracted primarily or exclusively to significantly younger persons' (Groth and Birnbaum 1978: 176). As already noted, during the course of the research reported here, an attempt was made to determine whether the 40 men in this sample could be classified as fixated or regressed offenders; and whether that would assist in understanding their offending. The outcome of that exercise is reported later in the thesis.

\section{Forms of abuse}

Child sexual abuse is usually categorised as either intra-familial or extra-familial; and either as contact or non-contact abuse (NSPCC 2013b). Here, the distinctions between the former are examined, beginning with intra-familial abuse. Each of the forms of abuse referred to in the section which follows are of relevance, as they are characteristic of the abuse experienced by - and also later perpetrated by - offenders in this research study.

\section{Intra-familial abuse}

Intra-familial abuse is defined as abuse perpetrated by those who are either related to their victim/s, or live with them (Smallbone and Wortley 2001). Much CSA is intra-familial. It occurs within the family home and is often perpetrated by males who are related to their 
victims. Cawson et al. (2000), for example, found that perpetrators in their research were in the main male family members. In over a third of cases (38\%), penetrative/oral acts were perpetrated by a brother or stepbrother. This is in contrast with research that suggests that female victims, in particular, are most at risk from fathers and stepfathers (Finkelhor 1984). In Cawson et al.'s study, just under a quarter (23\%) of the abuse was perpetrated by the former; $14 \%$ by an uncle; $13 \%$ by a stepfather; and $6 \%$ by a grandfather. Abuse was perpetrated by females in only a small number of cases ( $4 \%$ by a mother).

Evidence suggests that females are more likely to be victims of intra-familial abuse (Finkelhor 1994; Fischer and McDonald 1998). This form of abuse is likely to last longer than extra-familial abuse due to the accessibility of victims; and the fact that victims are less likely to disclose (ibid.). It is also more likely to involve more serious forms of sexual activity (Russell 1983); and to progress more quickly to those activities than extra-familial abuse (op.cit.).

\section{Extra-familial abuse}

Extra-familial abuse is defined as that perpetrated 'outside family settings' (Smallbone and Wortley 2001: 2). Abusers include friends of the family; neighbours; those in positions of trust; and strangers (Fischer and McDonald 1998). Extra-familial abusers are more likely than intra-familial offenders to abuse boys (Finkelhor 1984); and to use force - either physical or verbal (Fischer and McDonald 1998). Older children are more likely to experience this form of abuse (ibid.); this may be due to the fact that they are less well-supervised than younger children and thus, are more readily accessed by abusers outside their own family.

Spiegel (2003: 142), highlights the sense in which an extra-familial abuser, in an attempt to build trust and enable access to their victims, will provide support with everyday activities such as babysitting or, for example, teaching a child to play football. Indeed, as part of their strategy: 'They stalk for an empty niche and seek to fill it'. Glasser et al. (2001: 492) make some useful observations regarding the characteristics and impact of extra-familial abuse, comparing it to intra-familial abuse, and these are worth noting here. They argue that cases of extra-familial abuse involve only the victim and abuser and thus, this form of abuse is 'less inclusive than incest'. The authors further posit that the victim may be groomed and 
'nurtured' by an extra-familial abuser; and that the abuse: 'is more likely to occur as a series of isolated events, unlike the more pervasive atmosphere of an incestuous family’.

They also shed some light on the vulnerability of victims to extra-familial abuse noting that some:

'are likely to suffer from deprivation, rejection or physical abuse within the family and to be looking for their social needs to be met outside the family. They are, thereby, easily drawn into intimate relationships with strangers or friends of the family who are seen as gratifying emotional needs that have gone unmet within the family. Such victims of abuse outside the family often feel helpless, unprotected, rejected or abandoned by the family'

The authors argue that this heightens the isolation experienced by the victim; increases their dependence on the abuser; and results in more intense shock and feelings of betrayal at the point of abuse. The victim is likely to feel 'more unprepared and betrayed than someone who has been abused within the family'. Indeed, there is a likelihood that the victim will have a 'greater need...to defend against the consequences of a more severe early trauma by identifying with the aggressor...and, in turn, becoming an abuser.' (ibid.)

Extending the discussion around difference between these forms of abuse, Spiegel (2003: 29) points out that extra-familial abusers who demonstrate a preference for male victims are more likely to 'align with the boy at his developmental level', thus 'fixating' on the 'role of a child'. Intra-familial abusers on the other hand, are more likely to 'elevate the child into the role of an adult'. The extent to which this might be the case for offenders in this sample, is explored in the Research Findings Chapters.

\section{Summary: Part 1}

In Part 1 of this Chapter, the problem of CSA has been placed in context. The challenges associated with defining CSA have been highlighted. Moreover, its prevalence within the general population and in sexual offending populations has been examined. In spite of an abundance of data, it remains the case that whatever form the evidence takes, only a partial account of CSA is provided. Official statistics are lacking in that much CSA is never reported. Moreover, it is clear from the research evidence, that attempts to determine the extent of the problem and to compare findings across studies are compounded by a range of methodological issues, including those related to definitions and variations in: samples; 
research locations; and the measures employed by researchers. Taken together, the result is conflict and contradiction on a number of levels. At the heart of this, is the prevailing nature of sexual offending against children as a well-hidden crime (Smallbone and Wortley 2001), which to some extent may remain secret because victims may not recognise an experience as abusive.

Clearly, the available evidence is characterised by a divergence which is unhelpful. There appear to be some consistent messages, however: namely, that females are more likely to experience CSA than males - the caveat here, of course, is that males are less likely to report abuse; and that sexual offenders are more likely to have experienced CSA than other offenders and those in the general population. Indeed, notwithstanding methodological issues, childhood sexual victimisation appears to be a: 'common experience for adult sex offenders' (Faller 1989: 543). Moreover, children and adolescents who engage in SHB also appear to commonly present with sexually abusive childhoods.

In the latter stages of Part 1, victims' characteristics were explored and the issue of vulnerability discussed. Then, the emphasis shifted to perpetrators and forms of CSA. Moreover, distinctions between intra-and extra-familial abuse were examined as a means of setting in context the abuse experienced - and perpetrated - by the 40 men in this research study. In Part 2 of this Chapter, attention shifts to female-perpetrated CSA. 


\section{Part 2: Female-perpetrated CSA}

\section{Introduction}

Five of the 40 men who participated in this research study reported at interview that they had been sexually abused in childhood by a female. A further 3 men had been abused at different points in their childhood by both males and females. The men's accounts of that abuse are presented in the Research Findings Chapters. Here, in an attempt to 'understand' the sexual violence experienced by those men, the focus is on evidence relating to female-perpetrated CSA.

Mendel (1995: 206) draws attention to the 'societal equation of males with oppressors'. Much of the research on sexual offending has been on male perpetrators and female victims, with CSA perceived as a 'male problem' (Bunting 2007: 253). However, an increasing body of evidence has emerged on FSOs in recent decades (Gannon and Rose 2008). Some researchers have focused on FSOs' characteristics (Csorba et al. 2005; Vandiver and Kercher 2004); others, on their sexual histories (Kaplan and Green 1995); differences between solo and cooffenders (Vandiver 2006), and child and juvenile FSOs (Tardif et al. 2005). Some have examined professional responses to FSO (Bunting 2007; Hetherton and Beardsall 1988); and the role played by the idealisation of women in minimising CSA perpetrated by females (Hetherton 1999).

A number of other studies have focused on maternal sexual abuse, for example, mother-son CSA (Banning 1989; Lawson 1993); adult male reporting of CSA by mothers (Krug 1989); and mother-daughter CSA (Peter 2006). Others have explored issues relating to victims' disclosure to professionals and the impact of professional response (Denov 2003); prevention (Oliver 2007); criminal justice intervention (Bader et al. 2008); and risk assessment (Turton 2010). In the midst of this emerging evidence, however, 'disagreement' persists as to how this form of abuse is defined; its frequency; and the 'characteristics' of FSOs (Tsopelas et al. 2011: 122). 


\section{The extent of female-perpetrated CSA}

\section{The problem of under-reporting}

Professionals' experience of female abusers is limited, and the policy and practice emphasis is on males as perpetrators (Turton 2008). McLeod (2015: 97) asserts, that as a result of societal failure to identify women 'as offenders', they are allowed 'to avoid detection, prosecution, and interventions'. Thus, only the most serious cases of abuse by women are brought to the attention of the CJS. These are most likely to include women as coperpetrators; therefore, a 'selection bias' results (Tardif et al. 2005). Official data indicate that the FSO population is small. Yet, difficulties encountered in determining the extent of this problem may arise from the gap evident between official statistics and victim-based information (Wijkman et al. 2010).

Some suggest, that as women are more likely than males to spend time with children with no other adult present, the small number of detected cases of FSO may be due in part, to the 'absence of adults who might report' (Faller 1995: 28). The care-taking role proves central to the masking of offending (Ferguson and Meehan 2005), as abuse 'can be concealed within a loving, caring, protective role' within which, access to children 'is easy and 'normal', (Etherington 1997: 110). According to Denov (2004:18) discourse around the extent of this problem is characterized by: 'under-recognition, uncertainty, and controversy.' Traditional sexual scripts have proved influential to thinking around FSO on a number of levels, evidenced by: the response of the CJS; professional responses; and the extent to which victims feel able to disclose. The result is a ‘culture of denial' (Denov 2003a; 2003b; 2004).

\section{Prevalence and incidence}

Some incidence studies (Kendall-Tackett and Simon 1987; Cupoli and Sewell 1988) point to between $1 \%$ and $4 \%$ of CSA as being perpetrated by females. Others, (Finkelhor and Russell 1984), estimate that $5 \%$ of the abuse of girls and $20 \%$ of the abuse of boys is perpetrated by women; and that female-perpetrated CSA accounts for between $1 \%$ and $20 \%$ of all sexual offences (Moulden et al., 2007; Grayston and DeLuca 1999). As is so often the case in research on sexual offending, variations emerge depending on whether data were collected from official sources, or were retrospective studies of victims involving self-report surveys 
(Peter 2008). Of course, variations can also emerge due to environmental differences. Finkelhor et al. (1988), for example, found a higher incidence of female-perpetrated CSA (40\%) in day care settings in the US. More recent data indicate that females are responsible for 4-5\% of sexual offences, with a 1:20 ratio of female to MSOs (Gannon and Cortoni 2010; Sandler and Freeman 2007).

Alternative data sources provide a useful insight into the scale of this problem. Data recorded by the UK organization ChildLine, for example, indicate that the number of children reporting sexual abuse by women has more than doubled in recent years. Of 6,763 calls made to ChildLine between 2005 and 2006, 5\% of female victims and 44\% of male victims specified that the gender of their perpetrator was female. Moreover, $16 \%$ of males and 3\% of females who called about sexual abuse cited their mother as the abuser (NSPCC 2007). In 2009, ChildLine saw an increase of 132\% in complaints of female sexual assaults, compared to a $27 \%$ increase in reports of abuse by males. Some 2,142 children reported they had been sexually abused by a woman, with 1,311 reporting sexual abuse perpetrated by their own mother (NSPCC 2009). Given that some evidence suggests that male victims are more reluctant to report abuse by a female (Krug 1989), it is interesting to note that boys were more likely to say they had been abused by a woman (1,722 cases) than by a man $(1,651)$.

Elliott (1993: 2) places emphasis on survivors' accounts of abuse. Following a KIDSCAPE National Conference on Female Sexual Abuse in London, a 'hotline' was opened by the television programme 'This Morning'. Over 1,000 calls were received from victims of female-perpetrated CSA, with some $90 \%$ of callers stating that they had never told anyone of the abuse. As Elliott noted at the time: 'Finding survivors willing to talk was no problem, finding people willing to listen was'. She cautions against an over-reliance on official statistics, and of reaching the conclusion that if it is not reported 'then it must not happen' (Elliott 1993: 2). Donald Findlater, of the Lucy Faithfull Foundation - a child protection charity specialising in sexually harmful behaviours - also cautions that the problem of FSO is likely to far exceed conviction rates. Indeed, in his view, it is possible that there may be between 48,000 and 64,000 FSOs (The Guardian 2009a). As Turton (2008: 13) notes, CSA perpetrated by females 'is hardly an insignificant matter'; yet, responses often take the form of denial, excuses, and minimisation. 


\section{Gendered constructions of sexual offending}

Etherington (1997) posits that fixed views of gender and sex roles have hampered our understanding of FSO. An inability to associate male with the status of victim or female with perpetrator (Watkins and Bentovim 1992) is in part, due to the more readily accepted view of maleness as characterised by domination and power, as opposed to weakness and vulnerability (Finkelhor 1984; Lawson 1993). The preferred emphasis on females as victims of crime emanates from their role as caregivers within society (Pritchard and Hughes 1997). Thus, academic attention has centred 'almost exclusively' on MSO (Wijkman et al. 2010: 135). Where women are recognized as sexual offenders, they are viewed 'either as monsters or victims' (Nathan and Ward 2001: 45); 'mad,' or 'bad' (Grattagliano et al. 2012: 181). Given this, it is essential that any discourse on FSO should include some reflection on the extent to which it is understood through a gendered lens (Denov 2004). In order to further understand the reluctance to position women as sexual offenders, the contribution of myth is explored next.

\section{The myths}

Glasser et al. (2001) argue that being female is one of the characteristics which serves to protect victims from becoming abusers. Traditionally, women are perceived as carers and nurturers and as sexually safe with small children. The myths associated with FSO serve to perpetuate the failure to recognize women's capacity to harm, namely, that: females do not sexually abuse; they only abuse if coerced by a male; such abuse is 'harmless', gentle, loving, or misguided 'motherly' love; and females only abuse boys or adolescents (Longdon 1993). Against a background of myth, research evidence affords an alternative account. The mean age of victims in Faller's (1987) study, for example, was 6.4.years; and the majority of victims were female. More recently, Mcleod (2015: 109) found that FSOs 'showed a distinct preference for female victims'. Moreover, in Bader et al.’s (2008) research, approximately $75 \%$ of the women were lone perpetrators, a finding consistent with some other studies (Nathan and Ward 2002). On harm, Bunting (2007: 264) highlights the 'very serious' nature of offences perpetrated by women, which: 'commonly involve violence and have a wide range of negative and far-reaching consequences for victims'. 
Media responses to sexual offending serve only to further confound understanding. Frei (2008: 495), for example, notes that newspapers in the US typically locate FSO within the teacher-lover dimension, with the female depicted as an: 'emotionally fragile or misguided woman who made a mistake with an under-age partner.' Victims in these cases are more likely to be referred to as 'willing participants'. This is also true of much media representation in the UK, with the abuse often described as an 'affair', as in the case of the female teacher imprisoned for sexual activity with a pupil (Daily Mail 2006). More recently in the UK, the case of a nursery worker who abused young children in her care, making and distributing indecent images through her mobile phone (The Guardian 2009b), has focused attention on the problem of FSO. However, the sensational manner in which this case was sometimes reported in the media has done little to aid understanding, with headlines denoting the woman's fall from grace; an emphasis often on the female abuser's attempts to please the male involved; her lack of agency; and the control exerted by a dominant male. Thus, it appears that attention is steered towards MSOs even where females are present at, and active in, the abuse (Turton 2010).

Misunderstandings as to the nature of FSO have grave consequences for victims, potentially placing them at considerable risk. The myths which abound inhibit understanding. Indeed, their very existence together with societal acceptance of them as reality, works to abusers' advantage by: 'silencing victims and encouraging public denial about the true nature of sexual assault.’(McAlinden 2006: 354).

\section{Categorising female-perpetrated CSA}

Child sexual abuse perpetrated by women ranges from: genital fondling; to oral sex; and sexual penetration (Vandiver and Kercher 2004; Vandiver and Walker 2002). It can include digital penetration and penetration with objects (Elliott 1993); touching or kissing genitalia; exhibitionism; and coercing a child to watch sexual activity (Denov 2003); voyeurism, rape and sadistic sexual abuse (Bunting 2005). Turton (2008: 28) makes the point that:

'The expectation of a close, loving bond between mother and child gives women a licence to be intimate; it also means that their motives are generally interpreted as benign'. 
Whereas close, adult, physical relationships often have a sexual element, mothers are defined as 'asexual' (ibid.). Lawson (1993), in research on mother-son CSA, distinguishes between 5 forms of maternal abuse: subtle; seductive; perversive; overt; and sadistic (these could, however, also apply to abuse perpetrated by other females).

Subtle sexual abuse is characterised by a lack of coercion; does not necessarily involve genital contact; and may result from the 'mother's own unconscious need for sexual gratification' (p265). Subtle abuse involves the inappropriate sexual stimulation of the child which is motivated by parental 'sexual needs', but does not involve force. Saradjian (1996) provides some examples of this form of abuse which may include: continuing to bathe a child beyond an appropriate age; excessive washing/cleaning of the child's genitals; sharing a bed with the child; and administering unnecessary enemas.

Seductive abuse is defined as inappropriate sexual stimulation of a child in order that the mother's sexual needs are met. Behaviour may include seductive and sexual posing; nudity; and introducing the child to pornographic material. Perversive abuse includes behaviour which is 'intended to emasculate and humiliate the child's sexuality' (p266), for example, being critical of a child's sexual development, or forcing a male child to wear female clothing (Saradjian 1996). Overt abuse is characterised by 'overtly sexualised contact between mother and son' (p.266), coercion and in some cases 'threats to discourage disclosure'. This form of abuse may range from intercourse; to fondling of the child's genitals; coercing the child to fondle the mother's breasts or genitals; cunnilingus; fellatio; digital penetration - or penetration with objects - of the child's vagina or anus; and sexualized touching and kissing (Saradjian 1996). Sadistic sexual abuse is perpetrated with the intention of hurting the child, for example by inserting objects into the child's vagina or anus; or beating, rubbing or cutting the child's genitals.

More recently, Denov (2003), classified the abuse - based on its severity - as follows:

Severe sexual abuse - for example, sexual intercourse; penetration (anal or vaginal) with objects; or digital penetration; fellatio; and cunnilingus

Moderate sexual abuse - for example, genital fondling (without penetration); and simulated intercourse 
Mild sexual abuse - for example, sexualized kissing

While such attempts to categorise this form of abuse are useful, our understanding as to what constitutes sexually abusive behaviour by females is compounded by the fact that women are permitted a much greater range of physical contact with children than men (Oliver 2007; Saradjian 1996). For example, many women bathe and dress children while others sleep in the same bed as a child. These behaviours are all viewed as 'normal' activities for women and perceived as more acceptable for women than for men. They are therefore much less likely to be seen as warning signs of abuse even though they can all lead to abuse (Oliver 2007). Thus, abuse is 'less easily detectable’ (Wijkman et al. 2010: 136). Elliott and Ashfield (2011: 93) draw attention to the benefits of these 'recognition barriers' to those who seek to abuse children: namely, 'easier access to victims and the ability to disguise abuse as child care'.

The categories proposed by Lawson (1993) and Denov (2003) are considered further in Chapters 8 and 9, where some thought is given to the extent to which they may be applied to offenders' accounts of their experiences of female-perpetrated CSA.

\section{Typologies}

While much emphasis has been placed on developing theoretical frameworks; and tools to aid in the assessment and treatment of MSOs, the same does not apply to FSOs (Gannon and Rose 2008). Notwithstanding this, some attempts have been made to classify FSOs through the development of typologies, (Vandiver and Kercher 2004), albeit at the expense of exploring ‘treatment requirements' (Gannon and Rose 2008: 442). These are briefly explored here.

The work of Matthews et al. (1989) is most often cited in relation to typologies. Three categories of offenders emerged from the work: namely, teacher/lover - where the emphasis is on teaching about sexuality, and offenders believe the behaviour is consensual, and a love affair; predisposed (intergenerational) - women who have been sexually abused frequently as children by multiple perpetrators; and male-coerced - women who are dominated by a male and who have themselves been subjected to verbal/physical/sexual abuse. The authors also 
proposed a fourth category: psychologically disturbed - women characterised by psychoses/psychological problems

Other researchers have further refined and expanded these categories (see Nathan and Ward 2001; Syed and Williams 1996; Vandiver and Kercher 2004; Wijkman et al. 2010). While typologies shed some light on FSO, they also have their limitations, namely the provision of only a 'broad' understanding of female perpetrators (Gannon and Rose 2008: 448). In light of this, typologies are not referred to outside this Chapter.

\section{Characteristics of FSOs}

As is the case with other populations of sexual offenders, FSOs are a 'highly heterogeneous group' (Oliver 2007: 21), who differ in terms of their characteristics; their choice of victim; and the context within which they offend (Sandler and Freeman 2007). They tend to be caregivers to victims: often they are mothers, other relatives or babysitters (Vandiver and Walker 2002; Wijkman et al. 2010); the majority are white (Vandiver and Kercher 2004); have few qualifications (Nathan and Ward 2001); and fall into the low to middle range in terms of socio-economic status (Lewis and Stanley 2000).

Some (Faller 1995) have found that over a third of FSOs were married. Others, however, report lower marital rates (Lewis and Stanley 2000). They often offend with one or more accomplices; those accomplices are usually, but not always, male (Nathan and Ward 2001; Vandiver 2006). Some argue that it is not likely that females will initiate abuse in the absence of a male co-offender (Grayston and DeLuca 1999); others posit that female offenders do abuse independently of others (Deering and Mellor 2011; Elliott 1993). In a comparison of solo offenders and co-offenders, Vandiver (2006) found co-offending rates to range from 22\% to 96\%. More recently, Wijkman et al. (2010: 135) found that two thirds of the women (63\%) in their study had offended with a co-perpetrator; in $75 \%$ of cases this was a husband or partner.

Some females target adolescents, others young children; some target boys, others girls; and others both sexes. In some cases, victims report the use of force or violence as part of the abuse (Deering and Mellor 2011). Notwithstanding this divergence, however, there are some characteristics which appear to be common to female abusers. It appears that they are more 
likely to be young women (Bourke et al. 2014); most range in age between 16 and 36 years (Vandiver and Kercher 2004), with a mean age of 26 years (Faller 1987).

The childhoods of many FSOs are marked out by physical and emotional abuse (Travin et al. 1990); and sexual victimization (Elliott et al. 2010; Strickland 2008). In some cases the latter has been found to be severe; to have begun at a very early age; and to have involved multiple perpetrators (Lewis and Stanley 2000; Wijkman et al. 2010). Many FSOs come from 'broken and dysfunctional families' (Wijkman et al. 2010: 138), and their adverse experiences impact on their capacity to form secure attachments in childhood (Nathan and Ward 2001). Many experience difficulty with relationships (Elliott et al. 2010); and are re-victimised as adults (Lewis and Stanley 2000).

Much of the research on SHB by children and young people has emerged in recent decades (Veneziano and Veneziano 2002); and focuses on adolescent males (Hickey et al. 2008; McCartan et al. 2011). Where research has been undertaken on females, it is often limited by small sample size; and an emphasis on a population often engaged in treatment (ibid.). Notwithstanding such limitations, many young females have been found to have experienced severe sexual and physical violence in childhood (see, for example, Fehrenbach and Monastersky 1988; Hunter et al 1993; Masson et al. 2015; Matthews et al. 1997). Adolescent females usually offend against young children, who are often under 5 years, and will typically have more than one victim. Victims are often known and will usually be relatives (Kubik et al. 2003; McCartan et al. 2011). There are both similarities and differences in relation to 'characteristics, backgrounds and behaviours', between young females and males who engage in SHB (Masson et al. 2015: 28). The former differ from adolescent MSOs in that the vast majority will have in their background, like adult FSOs, evidence of family dysfunction; and as already noted, a history of childhood sexual victimization which is often severe and protracted (Hackett 2014; Hickey et al. 2008; Masson et al. 2015; McCartan et al. 2011).

Some have found that these females are more likely to present with learning difficulties (McCartan et al. 2011). Others, point to the inadequacy of 'sexual boundaries' within the family home, and resulting inappropriately early sexualisation (Hickey et al. 2008: 241); and a 'significantly earlier onset' of SAB when compared with males (ibid.: 249). Unlike adult FSOs, adolescent females do not usually sexually abuse with a co-offender (Fehrenbach and Monastersky 1988; Kubik et al. 2003). It appears that they are unlikely to have problems with 
schooling (ibid.); and to engage in aggressive and violent behaviour, and other anti-social activities such as criminal damage; and substance misuse and self-harm (McCartan et al. 2011). Of note, is that the distinct and different nature of this population - when compared with adolescent MSOs and adult FSOS - warrants further more detailed attention, given the implications of such difference for assessment and intervention (ibid.).

\section{Distinctions between male and female child sexual abusers}

Clearly, FSOs share many characteristics with MSOs: they are known to their victims and select those they have easy access to; they may take up employment in settings involving children; or offend because opportunity presents (Vandiver and Kercher 2004; Vandiver and Walker 2002). Moreover, some evidence suggests that like MSOs, female abusers exhibit empathy deficits; poor social skills; low self-esteem; and emotional loneliness (Saradjian 1996). Research evidence also suggests that FSOs frequently experience intimacy deficits with adults, finding safety, intimacy and love only through sexual activity with a child. Some, however, report perpetrating abuse in order to achieve a sense of closeness with their cooffender, rather than with the child victim (ibid.).

There are also marked differences between these two offending populations (McLeod 2015). FSOs are likely to be younger than MSOs (Bourke et al. 2014); more likely to abuse with a co-perpetrator who is usually - but not always - male (Vandiver 2006); and unlikely to force others into co-offending (Matthews 1993). They are also less likely to misuse substances or alcohol at the time of the abuse (Lewis and Stanley 2000) or when compared to other female offenders (Kubik et al. 2002). Some evidence points to FSOs being less likely to use force during the abuse than MSOs (Grayston and DeLuca 1999). However, others have found there to be no difference between male and female abusers in terms of their use of force (Mathews et al. 1997).

The backgrounds of FSOs - like those of MSOs - appear to be 'abusive and chaotic' in nature (Gannon and Rose 2008: 445). However, of note, is that the CSA experienced by FSOs appears to be more frequent and severe than that experienced by MSOs (Oliver 2007). Indeed, Strickland (2008) suggests that the severity of that abuse may prove significant in the development of a sexual interest in children. Others, too, purport that these experiences may provide a partial explanation for the low self-esteem, and passive, dependent nature of FSOs; 
and their propensity to engage in abusive relationships as adults (Gannon and Rose 2008). Given such distinctions, it is imperative that attempts are made to understand: 'the gendered patterns in child sexual offending behaviors' (McLeod 2015: 99). In an attempt to do so, distinctions are made in this thesis between the male and female-perpetrated abuse experienced by the men who participated in this research.

\section{Victims}

Victims of female perpetrated CSA are most likely to be known to the abuser, or related to them (Vandiver and Walker 2002; Wijkman et al. 2010); and young, on average less than 12 years old (Lewis and Stanley 2000). Some research points to a higher proportion of female victims (Peter 2009); in other studies, all, or the majority of victims have been male (Lewis and Stanley 2000). While some research indicates that lone perpetrators are more likely to abuse males (Bourke et al. 2014), others have found that they have abused both male and female victims (Vandiver and Kercher 2004). Vandiver (2006) suggests that solo offenders are more likely to abuse males; and co-offenders, female victims. More recent research findings from a study undertaken in the Netherlands by Muskens et al. (2011) - albeit with a smaller sample - are consistent with Vandiver's (2006). Tardif et al. (2005) report some interesting distinctions in terms of victim gender in their sample of adult and juvenile female abusers. Here, the majority (76.9\%) of adults abused female victims; the majority of juveniles (60\%) abused male victims. Johnson (1989) also found that the young females in her sample were more likely to select male victims: abusing twice as many boys as girls. More recently, Masson et al. (2015: 27) reported that young females were more likely to have: 'fewer victims drawn from a more narrow age range'.

\section{Summary: Part 2}

Child sexual abuse is an emotive topic. This is compounded where the abuser is female, for acts of sexual violence are assigned to males (Turton 2008). Indeed, female abusers are often consigned to a status of having been coerced by a male; of colluding in the abuse; or alternatively, they are perceived as ‘disturbed' (ibid.: 119). Understanding - and locating female-perpetrated CSA presents many challenges, not least those associated with prevailing stereotypical images of masculinity and femininity. It has been explored here, as a means of placing in context offenders' experiences of this form of abuse. Five of the 40 men who 
participated in this research had experienced female-perpetrated CSA; a further 3 reported abuse by both males and females in childhood. The extent to which the findings of previous research on this topic apply to these 8 men is considered in the Research Findings Chapters. Here, the myths associated with this form of offending have been examined, alongside the gendered nature of constructions of offending; evidence as to the extent of the problem; and the challenges encountered in defining FSO. The characteristics of female abusers were considered, and developments in classifying FSOs highlighted. The emphasis then shifted to victims.

In Part 3 of this Chapter, issues relating to disclosure and the silencing of victims are examined. 


\section{Part 3: Disclosure and the silencing of victims}

\section{Introduction}

Difficulties encountered in understanding the extent of the problem of CSA stem, in part, from the fact that many adults report never having disclosed abuse during childhood (Alaggia 2004), and it is suspected that many never disclose at all (O’Leary et al. 2010). Some, particularly children with disabilities, are even less likely to disclose than those in the general population (CCE 2015). Of course, what should be acknowledged at the outset of any discussion on this topic, is that if victims are to disclose, they first need to recognise that they have been sexually abused (Schonbucher 2012). Attention has been drawn to the benefits of disclosure in childhood; and child protection agencies place emphasis on the need to tell as soon as possible (Alaggia 2004). Yet, the potential for responses to disclosure to impact negatively on the victim must also be acknowledged, for a hostile or non-supportive response can have consequences for victims' long-term mental health (Feiring et al. 2002); and 'longterm recovery from the effects of abuse’ (Fontes and Plummer 2010: 491).

The concept of disclosure is of particular relevance to this thesis because: first, it is such a central component in the victimisation process; second, because so many of the offenders in this study chose not to tell about the abuse they experienced in childhood; and third, because so many others reported experiencing negative responses when they did tell. There is a dearth of research on the concept of disclosure as it relates to child sexual abusers who experienced sexual victimisation in childhood. Given the power of appropriate response to disclosure to aid recovery from abuse, it is crucial that some attempt is made to understand abusers' experiences.

\section{Disclosing abuse}

The first thing to note is that CSA: 'is largely a silent and witness-free crime, often leaving no physical signs and actively hidden by perpetrators' (Allnock 2010: 1). Thus, detection proves extremely difficult: 'with increasing importance placed on the victims' disclosure of abuse for investigative and treatment purposes’ (ibid.:1). However, as Gilligan and Akhtar (2006) note, the impact and meaning of CSA will differ, according to victims' gender; education; 
class; age; religious beliefs; and origins. Each of these will in turn impact on the likelihood that a victim will, or will not, disclose.

Radford et al. (2011) found that more than one third (34\%) of children aged 11-17 years who had experienced contact sexual abuse by an adult perpetrator did not disclose that abuse. In addition, 4 out of 5 (82.7\%) children aged 11-17 years who had experienced contact sexual abuse perpetrated by a peer did not disclose. The most recent data available from the CSEW (2016), indicate that 3 out of 4 victims of sexual assault by rape or penetration during childhood did not disclose the abuse. Amongst the most often cited reasons for this were: humiliation; embarrassment; and a fear of not being believed. Moreover, in a recent survey of adult survivors of CSA (Smith et al. 2015), 70\% of those who participated in the study had not reported the abuse. Where disclosure does occur in childhood, close friends of the victim - and in some cases, mothers - rather than professionals or other adults, are often privy to that information (Schonbucher 2012).

Disclosure of CSA is a 'complex process' (Priebe and Svedin 2008: 1095). Yet, such complexity is rarely understood or acknowledged. Disclosure is not a singular event (although it is often portrayed as such). Rather, it is a process which can be prolonged and is characterised by diversity; guilt; relief; anger; and pain (Durham 2003: 317). For many victims, much of that pain stems from the shift of information which was private into the public arena, as professional attention is drawn to: 'intense personal conflict and turmoil' (ibid.: 316). A further complication arises for victims as part of the process of telling: for, in doing so, they are labelled as a victim (Lamb 1999), a status not welcomed by many.

Secrecy permeates CSA (Leonard 1996). The isolating nature of abuse contributes to the child's lack of 'reference standards with which to judge the experience'; the perpetrator is therefore free to 'assign meaning' to the abuse and create a 'reality' which serves to maintain offending (ibid: 110). Sgroi (1982), identified two forms of disclosure: accidental and purposeful. The former is characterised by 'chance' rather than a deliberate attempt on the victim's part to tell; the latter involves a conscious decision by the victim to disclose abuse. Alaggia (2004) points to another form of disclosure, namely: prompted or elicited disclosure; this occurs when parents, carers, professionals or other adults encourage a child who is reluctant to tell, to disclose abuse. The author also draws attention to the potential for children's behaviour and indirect verbal communication to act as a form of disclosure. 
However, the potential for the latter - indirect telling - to be misconstrued by those in a position to protect has recently been highlighted (CCE 2015: 10); in particular, a lack of understanding when children express 'discomfort or unhappiness' at the thought of spending time with an individual.

Others have focused on the stages children pass through during the process of disclosure, including: denial and accidental disclosure (Sorensen and Snow 1991); self - where children reach an understanding that they have been victimised and how they feel about that; confidant selection-reaction, and consequences (Staller and Nelson-Gardell 2005). While such attempts to determine the shape of disclosure provide us with some useful reference points, of course, the process is: ‘unique to each victim' (Lovett 2004: 355).

In recent decades, there has been an increased research emphasis on the issue of disclosure (Staller and Nelson-Gardell, 2005), albeit as it relates to female victims (Easton 2013). Researchers have focused on: factors which influence children to self-disclose (Paine and Hansen 2002); and variables associated with delay of disclosure (Goodman-Brown et al. 2003; Smith et al. 2000). Others have examined children's perspectives on disclosure and the context within which it takes place (Jensen et al. 2005); health professionals' responses to disclosure (McGregor et al. 2010); adolescent victims' perceptions of impediments to disclosure (Bascelli et al. 2004); and how mothers ‘discover’ abuse (Plummer 2006).

A number of factors have been identified as motivating victims to disclose during childhood and adolescence: namely, anger towards the perpetrator; peer support; educational programmes which highlight inappropriate behaviour (Sorensen and Snow 1991); and the provision of sex education by individuals who are comfortable talking about sex (Fontes and Plummer 2010). Disclosure is also facilitated if: victims are older; the abuse is extra-familial; and there is a positive relationship with parents (Priebe and Svedin 2008). Goodman-Brown et al. (2003), propose that two factors are central to children's decisions to disclose abuse: first, the extent to which they believe others will tolerate disclosure; and second, the extent to which they perceive they are responsible for the abuse. Issues relating to a sense of shame and potential claims of 'complicity' (ibid. p.537) in the abuse feature in perceptions of responsibility, and serve to inhibit disclosure. Moreover, intra-familial abuse is likely to result in delayed disclosure due to the close relationship between abuser and victim 
(Goodman-Brown et al. 2003; Smith et al. 2000); and victims' fears as to the consequences for the family of disclosing abuse (Allnock 2010).

Some (Gilligan and Akhtar 2006: 1363) have explored cultural barriers to disclosure and found that fears about professional responses are exacerbated by cultural obligations to family relating to izzat (honour/respect); haya (modesty); and sharam (shame/embarrassment). The emphasis here is on the collective as opposed to the individual; and on protecting the community or family from the harm that might result from disclosure (ibid.). While all the offenders whose accounts are presented in this thesis were white and British, some parallels may be drawn between their accounts and Gilligan and Akhtar's (2006) concept of the collective need to protect family from the harm which might result from disclosure. This issue is discussed further in the Research Findings Chapters.

Children abused by educators (Roberts and Vanstone 2014); and those in positions of trust (Colton et al. 2012) are less likely to disclose. Issues of power and status feature whenever CSA is perpetrated. Where an abuser is a respected member of the community - for example, a teacher - the child's sense of powerlessness is further compounded (Moulden et al. 2007). Victims' gender also impacts on the extent to which they are likely to disclose (Alaggia 2004). Some evidence (Maikovich-Fong and Jafee 2010; Paine and Hansen 2002) suggests that females are more likely to report CSA and to have those reports validated. In male victims, however, 'non-reporting is particularly severe' (Finkelhor 1989: 233). Given that the focus of this thesis is upon males as victims - as well as perpetrators - of CSA, this issue is of particular interest. Societal myths around what is means to be male have contributed to victims' silence (CCE 2015; Finkelhor et al. 1990). The 'male ethic', of self-reliance appears to play a part in minimising victimisation and under-reporting of CSA of boys (Finkelhor 1984: 152). Moreover, the 'stigma of homosexuality' (ibid.) which surrounds CSA also features. These issues are further explored in the Research Findings Chapters, where offenders' accounts of disclosing their own childhood sexual victimisation - and their reasons for not doing so - are examined in detail. Next - and in the spirit of exploring difference - the focus is on the challenges victims face in disclosing female perpetrated CSA. 


\section{Disclosing female-perpetrated CSA}

There is a prevailing assumption that women do not engage in CSA; and, in particular, that they do not do so of their own free will (Turton 2008). While disclosure proves difficult for all victims, where abuse is perpetrated by a female there is: 'an added complexity given that these cases transgress the norm' (Denov 2003: 311). Indeed, the taboo associated with FSO (Denov 2004) inhibits disclosure; and for victims of maternal abuse, the situation is compounded (Turton 2013). According to Elliott (1993: 1): 'secrecy, distress, anger, controversy and fear surround the issue of female sexual abuse'; and victims' accounts illustrate how difficult they have found the process of disclosure (Deering and Mellor 2011; Denov 2004). Participants in Denov’s (2004: 167) study, for example, reported experiencing 'intense anger as a result of not having their story believed by professionals'; self-blame; questioning or denial of their experience; silencing and secrecy; and a lack of faith in professionals. Taken together, this results in 'secondary victimisation' (p.171). This is of concern, given that unsupportive responses have the capacity to 'exacerbate the negative effects’ of abuse and also contribute to under-reporting (Denov 2004: 187).

The victims who participated in Turton's (2008: 124) research - like so many others - also experienced difficulty disclosing abuse. Some believed that even those adults they trusted and loved would find their accounts of their victimisation 'too abusive'; and others thought that no-one would believe they had been abused by their mother. For Turton (2008: 39), stereotypical views of the 'ideal mother' as nurturer and carer confound understanding, and serve to 'silence' the voices of victims. Moreover, societal norms which endorse and even glorify older woman/younger boy relationships and fantasies (Hunter 1990) may also inhibit disclosure. Cases involving adult females and adolescent boys, for example, may be unreported because of the tendency, on the part of society and the victim, to view them as an ‘initiation' (Elliott 1993) into the realms of sexual activity, or a rite of passage (Mellor and Deering 2010).

\section{Summary: Part 3}

In this section of the thesis, the emphasis has been on disclosure and the silencing of victims: a key feature of CSA. Children make conscious decisions around telling, interpreting - and reinterpreting - their position in relation to others, in their search for those who may provide 
an appropriate response. Where the perpetrator is female, the child's task is compounded by: 'gendered social structures that obscure women who sexually abuse'; and a resulting sense of 'disbelief' (Turton 2013: 86). Jensen et al. (2005: 1395) refer to disclosure as a 'fundamentally dialogical process' and suggest that there are 3 central tenets to the disclosure process - opportunity, purpose and connection. Here, disclosure:

'becomes less difficult if the children perceive that there is an opportunity to talk, and a purpose for speaking, and a connection has been established to what they are talking about.' (p.1395)

Of course, part of the problem here, is that children often have difficulty in finding situations where there is enough privacy or prompts to enable them to share experiences. Moreover, children are extremely sensitive to others' reactions, and fearful that their disclosure might be misinterpreted (ibid.). There is also another point to note on this: for victims of maternal abuse, opportunities to talk may be further limited by an inability to 'trust'; thus, disclosure becomes 'a risk too far' (Turton 2013: 88).

Durham (2003: 317) asserts that the consequences of telling can have 'fundamentally farreaching consequences', because all is made public and subject to scrutiny. Unfortunately, the impact of this on victims is not always acknowledged. Indeed, often, particularly when high-profile cases such as the Jimmy Savile case in the UK emerge in the media, one of the first questions asked is: why did victims take so long to come forward with allegations? Posing this question demonstrates a lack of understanding and knowledge of the process of sexual victimisation and its capacity to silence victims; and is a disservice to victims who might not feel able to tell, might not know who to tell, and who might - for a variety of reasons - take the decision not to tell.

Some victims who have the courage to tell are not believed - the impact of this is considerable (Staller and Nelson-Gardell 2005). Others, are under extreme pressure to remain silent about the abuse they experience (Goodman-Brown 2003). Indeed, instilling fears around telling are a central feature of abusers' strategies, with abusive relationships carefully manipulated in order to silence victims (Durham 2003; Roberts and Vanstone 2014). Clearly, children find the process of telling difficult, and in not telling they place themselves - and possibly others - in situations where victimisation: is likely to continue; may increase in severity; and cause further harm. If such harm is to be prevented, it is crucial that there is a 
greater understanding of children's needs in terms of disclosure: in particular, an awareness of the processes children move through in their journey towards disclosure; and recognition of the importance of the concepts: opportunity, purpose and connection (Jensen et al. 2005).

In the next Chapter, the focus shifts to the impact of CSA. 


\section{Chapter 3: The impact of CSA on victims}

\section{Introduction}

Child sexual abuse: 'has a significant impact on the lives of victims and survivors' (CCE 2015: 12). Over many years now, there has been much research emphasis on understanding impact (Wilcox et al. 2004). Yet, little attention has been paid to the impact of childhood sexual victimisation on those who are the focus of this research: child sexual abusers. Indeed, much of the research on impact has focused on female survivors of CSA (Fleming et al. 1999); college (Briere and Runtz 1988); and clinical samples (Bromberg and Johnson 2001); and made use of standardised measures and checklists (Fleming et al. 1999). There are limitations to such an approach, not least, the exclusion of the individual experiences of others, including males who have been subjected to CSA. Moreover, a reliance on student samples limits the extent to which findings might be generalizable, as these populations might be more resilient and well-adjusted than other survivors (Oaksford and Frude 2003). Clinical samples, on the other hand, contain individuals who may have experienced more severe forms of abuse; and who may have also experienced some difficulties prior to abuse (Bromberg and Johnson 2001).

This Chapter is presented in 2 parts. In Part 1, the focus is on the diverse nature of impact; variables associated with more negative consequences of CSA; re-victimisation; impact and male victims; a positive impact of CSA; the impact of female-perpetrated CSA, and positive perceptions of that; and criminal behaviour as a consequence of CSA. In Part 2, discussion of the latter is extended to include sexual offending; and the pathway from victim to victimiser is examined.

\section{Impact: Part 1}

An increasing emphasis on the consequences of CSA for victims, has indicated that a 'wide range of problems' are evident (Roberts et al. 2004: 526): including PTSD; suicide; depression; anxiety; low self-esteem; substance abuse; difficulties with relationships; and sexual problems (Allnock et al. 2009; Browne and Finkelhor 1986; Colton et al. 2002). Other researchers have pointed to: poor physical health resulting in increased levels of contact with health services; high levels of sexual risk-taking behaviour; low levels of life satisfaction; and 
dependence on welfare (Fergusson et al. 2013); guilt, sexual promiscuity, running away, nightmares, and self-harming (Conte 1987); suicidal ideation (Calder et al. 2010); and eating disorders (Romans et al. 2001; Smolak and Murnen 2002). Kendall-Tackett et al. (1993: 164) highlight the diverse nature of impact; cautioning that it is not possible to identify a 'specific syndrome' related to experiencing CSA, or a 'single traumatizing process'.

Much of the research on impact has focused on female, adult survivors and has adopted a cross-sectional approach (Fleming et al. 1999). Female victims of CSA are reported to subsequently experience a fear of men (Jehu 1988); low self-esteem, and over-dependency (Burkett 1991). In a recent review of the literature, de Jong et al. (2015: 185) point to the negative outcomes of CSA which persist into adult life: namely, lower educational attainment; lower incomes; increased likelihood of engaging in criminality; negative experiences of parenting; and an increased likelihood of using violence towards their children; or alternatively, adopting a 'permissive' parenting approach. Sexualised behaviour appears to be more often evident in abused as opposed to non-abused children (Bromberg and Johnson 2001), with some suggesting that it occurs in between a quarter and a third of all sexually victimised children (Hall et al. 2002). It is important to note, however, that the majority of victims do not exhibit this form of behaviour; and that it has also been evidenced in children who have been not been sexually abused (Kendall-Tackett et al. 1993).

Mediating factors in the victim's life compound the task of establishing the long-term consequences of CSA (Craissati et al. 2002). Some children are ‘traumatized' by CSA; others experience 'milder' effects which are short-lived, and; and some present with little or no effects. Walsh et al. (2010) found that between 10\% and 25\% of victims did not experience psychological difficulties as children; and between $20 \%$ and $40 \%$ of victims did not exhibit symptomology in adulthood. There are, however, a number of factors which might play a part in this, including the characteristics of the abuse and the perception that it was not severe; denial by the victim; the child's ability to cope with experiences others would find traumatic; or environmental factors such as the presence of a support network (Widom and Ames 1994). Moreover, Lev-Wiesel (2000) posits, that children who place responsibility for the abuse on the perpetrator as opposed to blaming themselves, experience less negative consequences.

Methodological issues might also feature here: for example, researchers might not measure a comprehensive range of effects; thus victims might be symptomatic on those not measured. 
Second, victims might not exhibit symptoms at the time of the research - perhaps because they were able to suppress those symptoms, or because they did not understand their experience as being abusive at that point. It is possible that at a later stage in their development children might recognise that they have been victimised and might then exhibit symptoms. Third, it is possible that some children are asymptomatic and 'truly less affected' (Kendall-Tackett et al. 1993: 170). They might have experienced a less damaging form of abuse; and may also be more resilient, having a greater range of support to draw on which enables them to recover from the abuse, for example, social and/or psychological support. On the latter, some (Lambie et al. 2002; Wilcox et al. 2004) have argued that children may be protected from the most severe consequences of abuse, for example, by a supportive and stable family background which enables them to develop resilience.

\section{Variables affecting consequences}

All forms of child abuse pose considerable 'threats to child health and well-being' (Currie and Widom 2010: 111). For Fergusson et al. (2013: 664), CSA 'is a traumatic childhood life event in which the negative consequences increase with increasing severity of abuse'. Evidence suggests that abuse is most harmful where: the victim is younger; has a close relationship with the abuser; the abuse involves penetration; persists over time; and is characterised by force (Browne and Finkelhor 1986; Finkelhor et al. 1990; Fleming et al. 1999). Others have pointed to the most damaging consequences resulting from the betrayal of trust which characterises the abuse; and the failure to listen to victims (Beitchman et al. 1991).

\section{Re-victimisation}

Increasing attention has been paid to the issue of re-victimisation in recent years. Yet, again, the focus has been on female CSA victims (Widom et al. 2008); there is a dearth of research on the re-victimisation of male victims. The fomer are more likely to experience revictimisation (Arata 2000; Barnes et al. 2009; Filipas and Ullman 2006; Jankowski et al. 2005), particularly domestic violence, and sexual assault (DiLillo 2001) in later life. Much research on impact points to victimization in childhood resulting in adult victimization. However, research undertaken by Boney-McCoy and Finkelhor (1995), expands understanding of the extent to which an episode of victimization in childhood might prove a 
risk factor for subsequent sexual victimisation in childhood. This issue is of particular relevance to this thesis, as so many offenders reported having been re-victimised during childhood. According to Boney-McCoy and Finkelhor (1995) the sexualisation of a child which occurs during CSA, may result in behaviour which places the child at further risk. This issue is further explored during the Research Findings Chapters.

\section{Impact and male victims}

The impact of CSA on males: 'remains relatively under researched in comparison to women' (O’Leary 2009: 471). Some argue that the lack of attention paid to males emanates from the view that such abuse is rare; and that where it does occur, it has little or no effect on male victims (Watkins and Bentovim 1992). Under-reporting of CSA perpetrated against boys also features here (Mendel 1995). Hunter (1990) suggests, that CSA of males is both unrecognised and under-reported, because males tend not to associate what happened to them as abuse. Other researchers (Finkelhor 1984; Pierce and Pierce 1985) have highlighted boys' vulnerability to CSA, and their reluctance to report it as resulting from: the sexualised nature of peer groups; the existence of male sex roles; a sense of shame; fear of stigma; little encouragement to report and seek out treatment; and homophobia. According to Johnson et al. (2006: 76) this proves unhelpful and ensures that CSA: 'of boys remains in the shadows and is not discussed openly, underscoring its stigmatization'.

Watkins and Bentovim (1992) reported males experiencing difficulties with - and anxiety over - sexual identity; and inappropriately attempting to demonstrate masculinity. Other research, points to higher levels of suicidal ideation in men who have experienced CSA (O’Leary and Gould 2009). Some evidence suggests that males exhibit more negative effects across time (Boney-McCoy and Finkelhor 1995). This may be due to developmental factors, as, for example, adolescents attempt to manage the process of 'growing up' and all its associated complexities, alongside dealing with the impact of CSA and its meaning for them (Spiegel 2003). Amongst the effects on males cited by Spiegel (2003) are: anogenital injuries; depression; emasculation; aggression; academic problems; substance misuse; fear of rejection; relationship difficulties; running away; and a desperate need for love and affection.

Mendel's (1995: xiii) research is of particular relevance to this thesis. His emphasis was on male survivors and the impact of CSA, as evidenced by data collected from questionnaires 
completed by 124 men, and 'semi-structured clinical interviews' with 9 of those men. Finkelhor and Browne's (1985) four traumagenic dynamics - stigmatization, betrayal, traumatic sexualisation, and powerlessness - were each apparent in Mendel's data. Participants felt stigmatized - and different - as a result of the abuse they experienced. Betrayal was a central theme in the interviews undertaken: survivors felt confused, and were left with an inability to trust others and engage in intimate relationships, particularly where the abuse was intrafamilial. Traumatic sexualisation was also 'prevalent' (ibid.: 202) in participants' accounts, and for some, this resulted in a decline in 'sexual drive'. A sense of powerlessness too, was 'pervasive'. Factors associated with the most negative outcomes for victims included the use of physical as well as sexual violence; and abuse perpetrated by a sibling. Of note, is that the men whose abuse took these forms were more likely to attempt suicide.

Almost all of Mendel's (1995: 205) participants experienced difficulty 'integrating their sense of themselves as men with their childhood experiences of victimization'. The sense of what it means to be male does not include feeling helpless and being passive, and his participants experienced conflict in coming to terms with having been 'acted upon', and as a result, left helpless. Self-blame was also evident in participants, who recognised that they had been unable to protect themselves. This concept is not unique to male victims as it is also experienced by females. However, Mendel argues that: 'male socialization and societal notions of masculinity make men even more vulnerable to self-blame’ (ibid.: 206).

Participants in Mendel's (1995: 210) research also expressed a sense of shame; and many reported considerable substance abuse, and other addictive behaviour, including 'sexual compulsivity'. The latter resulted from learning which associated sexual activity with the means to achieve intimacy and demonstrate affection. Moreover, almost all expressed concern that having experienced CSA, they might subsequently become a perpetrator. For some of those men, that concern was so pronounced that they made the decision never to have children of their own.

Durham's (2003: 309) work is of particular methodological relevance to this thesis as he places emphasis on: 'the importance of survivors' perspectives and of allowing [them] to speak for themselves and theorize their own experiences.' He drew on a small sample of males aged 15 to 24 years to examine the experience and impact of CSA. The following 
effects are evident in those young men's accounts: anxiety and fear; a sense of anger; petty offending; difficulties with family relationships; feelings of isolation; the use of substances drugs and alcohol - to erase memories of the abuse; 'and unimaginable depths of deep private sadness'.

\section{CSA: A positive impact?}

Victims' assessment of their childhood sexual victimisation appears to influence their perceptions of it (Bromberg and Johnson 2001). There are a number of features associated with abuse which result in more negative perceptions: for example, self-blame and guilt on the part of the victim; and the use of force in the abuse (Coffey et. al 1996). While the majority of those who have experienced CSA report negative consequences, some victims report a positive impact. Some 7\% of female students in Finkelhor's (1979) study, for example, found the sexual experience positive. However, of note, is that only half of those experiences were with adults; the remainder were with other children who were at least 5 years older than the respondent.

Others have found that victims who believe the sexual activity is reciprocal and mutual, as opposed to coercive; and experience 'physical pleasure or sexual arousal' are more likely to hold positive perceptions of the abuse ((Durham 2003: 322). Briggs and Hawkins (1996: 221) focused on 84 imprisoned males - from 7 prisons in Australia - who had experienced CSA and subsequently became child abusers, comparing them to 95 males who had been sexually victimised but were non-offenders. There were some interesting distinctions between the 2 groups. The child abusers were more likely to have been abused by a female (50\%), often acting alone; and a larger number of people. They were also more likely to perceive the abuse as normal (88\%); to have liked some aspect of the abuse (69\%); and to report little negative impact in spite of the presence of a range of psychological problems, including low selfesteem, chronic ill-health, problems with adult relationships, and poor educational attainment. Of note, is that they: 'discounted the effects of abuse on their own lives [and] also discounted the damage they did to others'.

Some (Widom and Morris 1997) suggest that boys may be more likely to perceive sexual activity with adults more positively, as a rite of passage; and girls, more negatively, as an invasion of their body. Bromberg and Johnson (2001) point out that once more, differences 
across studies emerge, according to the location and nature of the research undertaken. Data collected in clinical settings are often characterised by negative perceptions of children's sexual activity with adults; data collected from non-clinical samples are marked out by a range of perceptions, including many which are positive or neutral.

\section{Impact and female-perpetrated CSA}

In both a popular and professional sense, CSA perpetrated by women is perceived as 'less serious' (Gannon and Rose 2008: 444), and relatively 'harmless', when compared with sexual abuse by males (Denov 2001). Saradjian and Hanks (1996) argue that because women are not expected to perpetrate sexual violence against children, victims experience more prolonged abuse; greater stigmatization; an increased sense of difference; and a greater sense of betrayal. Consequently, victims are unable to engage in healthy relationships in adulthood, and some may become abusers themselves.

Denov (2004) found that both males and females experienced a range of problems following abuse, namely: substance abuse; self-harm; suicidal tendencies; depression; anger, and fantasizing about harming the perpetrator; difficulties with relationships with women; difficulties with self-concept, a sense of identity, sexuality, and sexual intimacy; and a fear of abusing their own children or replicating their sexual victimization with others. Of note, is that the majority of respondents who had experienced 'severe sexual abuse' by mothers (p.1137) reported it as 'more harmful and detrimental' (p.1152) than similar abuse by men' (p.1153). Both male and female victims in Deering and Mellor's (2011: 58) research reported 'persistent and distressing effects' post abuse, in particular, emotional, psychological and social difficulties both in childhood and as adults. Others, too, place emphasis on the 'more damaging' nature of maternal sexual abuse, which is characterised by a shattered trust 'that is not easily restored' (Peter 2008: 1046; 2006).

\section{Positive Perceptions}

Some research points to positive perceptions of this form of abuse by some victims. Two of the men in Etherington's (1997: 113) sample, for example, experienced maternal abuse. They 'directly acted out their abuse' as children or adolescents; and both viewed the abuse they had experienced positively, as 'normal' and 'loving'. In Kelly et al.'s research (2002: 425), 
maternal abuse was characterised by 'subtle' behaviours not easily distinguished from caregiving activities. Of interest, is that half of the men who had been abused by their mother had positive or mixed views of the abuse they had experienced. However, there was evidence of increased levels of interpersonal problems in this population. Thus, the authors conclude that mother-son incest is 'particularly damaging' (ibid. 435).

\section{Impact: from victimisation to offending}

A growing body of evidence is emerging on the issue of whether CSA is an antecedent for deviant and criminal behaviour in both adolescence and adulthood (Spano et al. 2010; Swanston et al. 2003). Of course, most victims of CSA do not subsequently engage in criminal behaviour (Widom 1995). Factors such as the victims' relationship with the perpetrator, and the nature and severity of the abuse experienced appear to play a part in this (Glasser et al. 2001). Moreover, as CSA is often perpetrated within the context of family life characterised by multiple problems, it proves difficult to distinguish the effects of unemployment; poverty; parental substance misuse and other forms of family dysfunction, from CSA (Widom 1995).

Research evidence indicates that those who have been sexually abused in childhood are at increased risk of perpetrating intimate partner violence as adults; and also violence against their own children ((Widom 1989). However: 'the intergenerational transmission of violence is not inevitable' (ibid.: 164). Females are less likely to exhibit violent or aggressive behaviour and are more likely to develop psychological problems. Of interest, is that some have found that males who were sexually abused in childhood and who were also part of a household characterised by domestic violence - which they witnessed - have been found to perpetrate the most severe forms of violence (Downs et al. 1996). Victims of any form of abuse or neglect are more likely to engage in criminal behaviour as juveniles and adults, than those who have not been victimised (Widom 1995). Some (Widom and Ames 1994; Widom 1995), report that those who experience physical abuse in childhood are more likely to perpetrate violent sexual offences such as sodomy or rape as juveniles.

Much research points to the part played by CSA in later sexual offending against children (Burton 2003; Johnson and Knight 2000). Beitchman et al. (1992), for example, note that male victims exhibit confusion in relation to their sexual identity, and a tendency to repeat 
their own abusive experience. Some argue that experiencing CSA can increase the risk of engaging in SHB in adolescence (Bromberg and Johnson 2001; CCE 2015). Watkins and Bentovim (2002), for example, found that 1 in 5 boys sexually abused in childhood later - in adolescence - sexually abused other children. Other research evidence, too, suggests that those who experience CSA are more likely to perpetrate violent and sexual offences as adolescents (Burton et al. 2002); and adults (Watkins and Bentovim 1992). Notwithstanding this, the majority of victims do not become adult offenders (Widom 1989).

The extent to which CSA might play a part in an individual 'becoming an abuser' is central to this thesis and is examined in some detail in Part 2 of this Chapter.

\section{Summary: Part 1}

In this section of the thesis, the issue of impact in all its complexity and diversity has been examined. It is clear that for some victims the impact of CSA is considerable. They can experience a range of deleterious consequences, including psychological and emotional difficulties and re-victimisation. Yet, some children - even where they have experienced severe forms of abuse - remain asymptomatic or recover well. Browne and Finkelhor (1986) note the controversy which persists in relation to impact, and others (Fleming et al. 1999: 157) also draw attention to the problems encountered in researching this topic positing that:

'the influence of CSA on adverse long-term effects is mediated and influenced both by the severity of the abuse experiences and by a range of family and social background factors. This makes the interpretation of the direct effects of abuse per se difficult'.

It appears that debate as to the outcome of CSA is fraught with complexity, with factors such as the characteristics of the abuse; the victim's perceptions of it; and the support provided to the victim post abuse all evident (Bromberg and Johnson 2001). Perhaps, in the midst of such complexity and debate as to why some victims appear to be adversely affected and others are more resilient, what should be more readily acknowledged is that there will be many 'silent' victims of CSA: 'who, despite their psychological symptoms, never seek professional help, appear well-adjusted, and are capable of coping’ (Oaksford and Frude 2003: 65-66). Their reasons for remaining 'silent' will be individual to them and beyond the reach of academic 
research and inquiry. Thus, our understanding of impact - like so many other aspects of CSA - can only ever be partial. 


\section{Impact: Part 2 - From Victim to Victimiser}

\section{Introduction}

Much energy has been expended on establishing the prevalence of CSA, particularly in sexual offending populations. While these data are useful to some extent, they are limited in that they: 'do not indicate etiology or causality' (Weeks and Widom 1998: 348). Unfortunately, such limitations inhibit the capacity of policy makers and those working directly with offenders and victims to make informed decisions about appropriate treatment interventions. In this thesis, the emphasis is upon moving beyond prevalence, and contributing to the knowledge base by focusing on, and understanding, offenders' individual experiences of CSA; their perceptions of that; its impact on them; and their views on the extent to which it played a part in their later offending. However, before the men themselves articulate their understanding of the extent to which there might be a link between their victimisation and their later offending, the evidence on the factors which might play a part in that process is explored.

\section{Making the link between victimisation and offending}

In attempts to explain sexual offending, commentators have noted that: 'there are no simple answers' (Ryan 2002: 891). Indeed, a range of factors, including 'biological, psychological, and interpersonal factors', have been identified as leading to sexual offending against children (Whitaker et al. 2008: 530). Bandura’s (1977) theory of social learning has prevailed in its explanation of the initiation and acquisition of much human behaviour; and it is viewed as: 'the key learning and behavioural approach used to explain the cycle' of CSA (Cossins and Plummer 2016: 6). The notion that abusive behaviours are learned through observation or experience has dominated hypotheses regarding the question 'Why do they do it?' (Ryan 2002: 891): one of the most often asked questions about sexual offending, particularly where offences are perpetrated against children. According to Bandura (1977), individuals model the behaviour of those with influence or authority, especially where that behaviour results in a favourable outcome. It is possible that children who are subjected to violence - or who witness it - may normalise such behaviour and endorse the use of violence to manage particular situations in adulthood. Researchers have consistently drawn on social learning theory in attempting to explain the cycle of CSA (Christopher et al. 2007), in particular, the 
similarities between the abuse perpetrated by offenders and that which they experienced themselves in childhood (Burton 2003; Simons et al. 2008). Laws and Marshall (1990) note, that some male child victims model the behaviour of their abuser. Moreover, others too, (see, for example, Felson and Lane 2009; Burton et al. 2002) posit that those who are sexually abused in childhood learn and justify abusive behaviour; and replicate their own abuse, becoming perpetrators in adulthood.

Cossins and Plummer (2016: 6) draw attention to the strengths of social learning theory by highlighting its capacity to demonstrate the 'influence' of childhood sexual victimisation on subsequent offending; and the extent to which it confronts the emphasis on abnormal and deviant behaviour by locating CSA within a 'broader social context'. However, the theory is limited in that it does not explain why all those who have been abused in childhood do not subsequently offend, having been exposed to, and having learnt from, abusive behaviour. Neither does it explain why those who have not experienced CSA, later offend in this way (ibid.).

Buchanan's (1996: 4) work focuses on 'intergenerational cycles of child maltreatment', and although there is a 'limited focus' on CSA, it warrants some attention at this point, given that there may be some similarity in the 'mechanisms of transmission'. Buchanan proposes that 4 cycles contribute - either directly or indirectly - to intergenerational child abuse: sociopolitical factors; cultural factors; psychological and biological factors. The first two cycles have an extra-familial dimension and a societal focus; the remaining two are intra-familial in character, and individually focused. Socio-political factors can have a considerable effect on parenting capacity, and the sense in which children remain safe. Indeed, both politics and policies can - in every geographical location - impact on that. According to Buchanan (1996) issues relating to discrimination; poverty; and the status of women are key here. On cultural factors, Buchanan recognises children's vulnerability to abuse on a global level, cultural differences in parenting, and the protective factors evident in those societies marked out by equality, sharing and co-operation. The author calls for greater understanding of cultural difference and a consensus as to what constitutes child abuse, in order that ethnocentric understanding of the 'meaning and origin of practices' (ibid.: 86) does not result in abuse which is more damaging than that inflicted by parents. 
On psychological factors, Buchanan (1996) suggests that there are a number of familial variables which are predictive of child abuse: for example, psychological problems; substance misuse; and low self-esteem resulting from previous abuse. Finally, it is proposed that some parents are more vulnerable in a biological sense - due to ill-health, for example to perpetrating abuse; and some children may be more vulnerable to abuse, due to the ineffective parenting which results from that.

It appears that a range of childhood adversities contribute to later sexual offending. Marshall and Barbaree (1990), for example, place emphasis on poor socialisation, and the part played by parental violence in the development of intense feelings of hostility and resentment: key elements in sexual offending. Others, including Marshall (1989) and Ward et al. (1995), focus on insecure attachment styles and intimacy deficits to explain sexual offending. Moreover, Lee et al. (2002: 75) highlight the following developmental risk factors: 'poor socialisation, problematic parent-child relationships, parental violence and child abuse.' The problem, according to Ward and Hudson (1998: 47), is the: 'ad hoc proliferation of theories that often overlap, and essentially neglect each others' existence'.

As already noted, the impact of CSA is variable. Clearly, in some cases, CSA can result in: 'debilitating mental and emotional distress that prevails throughout adulthood.' (Simons et al. 2002). Indeed, Craissati et al. (2002: 236) note that if 'unresolved', CSA: 'together with a range of other childhood difficulties may lead to difficulties in emotional well-being in adulthood and problems with intimate relationships.' Other researchers (Browne 1994; Finkelhor 1986) argue, that where this is the case, there is a risk that CSA is perpetrated, particularly against males. This may be due to inappropriate patterns of arousal, or a means of repeating and resolving the offender's own childhood victimization experience.

Evidence as to the existence of a 'cycle' of abuse, whereby the victim becomes the offender is limited and conflicting (Plummer and Cossins 2016). Such conflict stems from prevalence data which indicate that females are more likely than males to be victims of CSA; yet males are more likely to perpetrate abuse (Cossins 2000; Simons et al. 2008). Craissati et al. (2002: 226) suggest that: 'approximately 1 in 10 victims are at risk of being convicted of a sexual offence in adulthood'. More recently, Ogloff et al. (2012) found that 5\% of male victims of CSA were later convicted of a sexual offence; for those men abused at 12 years of age or older, the conviction rate was 9.2\%. Hanson and Slater (1988: 486) caution, that the 
relationship between CSA and subsequent sexual offending is not 'specific'. Indeed, all forms of childhood abuse can result in 'behavioral and psychological problems in adulthood' (ibid.). Others concur, noting that attention should be paid to the fact that CSA: 'often occurs in the context of multiproblem homes, and sexual victimization of children may be only one of these problems' (Widom and Ames 1994: 305). Given this, it is crucial that the contribution of other factors associated with the family and the environment within which childhood is experienced are considered in relation to the impact of CSA: namely, dysfunction; parental substance abuse; and poverty (ibid.). For, negative childhood experiences, whatever their shape and form, render individuals vulnerable to an increased risk of sexual offending (Jespersen et al. 2009; Ryan 1998).

While some research links sexual offending in adulthood with childhood sexual victimisation (Simons et al. 2008), others argue that individuals who have experienced CSA are not more likely to perpetrate CSA as adults (Widom 1996). Thomas and Fremouw (2009: 386) posit, that while it is only one of the damaging effects of CSA: 'the victim to offender cycle is of major importance. It not only affects the original and future CSA victims, but is also a serious social concern.' Such concern is evident on a number of levels to a range of individuals and organisations: those who work in a therapeutic context with sexual offenders - either in the community or within the prison environment - and whose efforts are focused on effective treatment and rehabilitation; policy makers, whose concerns centre on the prevention of abuse, and a protection and safeguarding agenda; academics in their search for further understanding of this problem; the public, who are fearful for their own and their children's safety; and above all, victims themselves, who often express concern following childhood victimisation about their propensity to perpetrate abuse as adults.

The extent to which there is a link between childhood sexual victimisation and the later perpetration of CSA has been the focus of much research for some time now (see, for example, Bagley et al. 1994; Briggs 1995; Briggs and Hawkins 1996; Falshaw et al. 1996; Hanson and Slater 1988; Rabalais 2003). However, much of the research on this topic has included the use of checklists, which are not sensitive to detailed, individual experiences (Garrett 2010). Little emphasis has been placed on the views of prisoners convicted of CSA on the sexual abuse they experienced in childhood; their perceptions of that; its impact; and the extent to which it might contribute to later sexual offending: the focus of this thesis. Moreover, little effort has been made to explore the differences evident within - and between 
- groups of these offenders in terms of their previous victimisation and subsequent sexual offending. This represents a considerable gap in knowledge; and therein lies the justification for this research.

If the optimum levels of protection and intervention are to be provided for children; and effective intervention and treatment is to be delivered to offenders, it is crucial that abusers' accounts are heard; and any such differences explored. Here, an attempt is made to do that by exploring the narratives of 40 imprisoned, convicted child sexual abusers, and focusing on difference where that exists. As Spiegel (2003: 99) notes, on the differential effects of CSA on victims: 'highlighting similarities and differences validates the authentic experiences of childhood sexual abuse - its dynamics and effects'.

Child sexual abusers are more likely to live in a dysfunctional family environment in childhood than those who do not abuse children (Messman-Moore and Brown 2004). As already noted, higher rates of reported childhood sexual victimisation are found within sexual offender samples than in the general, and offending populations (Jespersen et al. 2009; Whitaker et al. 2008). Childhood sexual victimisation is a 'common experience for adult sex offenders' (Faller 1989: 543), with some clinical findings further suggesting that the age group of victims accords with the age at which the offender was himself abused as a child (Glasser et al. 2001). However, the issue is complex and the subject of much debate. First, due to concerns that abusers might report CSA and link that to their behaviour to explain their offending, minimise their guilt, access therapy, or evoke a sympathetic response which might work to their advantage on sentencing (Craissati et al. 2002; Glasser et al. 2001; Hanson and Slater 1988; Simons et al. 2002). On that, however, Lee et al. (2002: 75) remind us while there is a possibility that sex offenders might 'over-report' childhood sexual victimisation, 'the evidence is equivocal'. Second, because reporting previous abuse does not necessarily demonstrate a causal link with later offending (Weeks and Widom 1998). Indeed, CSA is often experienced in conjunction with other abusive behaviours, for example, neglect, and violence within the family. Thus: 'experiencing sexual abuse may be part of a more generally adverse early environment that is related to multiple problems later in life.' (Jespersen et al. 2009: 180). Third, because - as already noted - the majority of victims are female, yet the majority of perpetrators are male (Hilton and Mezey 1996; Glasser et al. 2001). Fourth, because clinical evidence suggests that individuals often repress, and subsequently do not 
recall, traumatic experiences (Hanson and Slater 1988). Finally, individuals may choose to deny that abuse was perpetrated (Faller 1989).

There appear to be many challenges to overcome then, in attempting to understand the problem of the link between victimisation and later sexual offending. Much of the problem emanates from the often conflicting nature of the available research evidence (Leach et al. 2016). Against a backdrop of obvious concern, a quantitative approach has prevailed with research focusing on sexual offenders, who are usually male, in a range of settings. A number of characteristics have been found to be associated with the risk of becoming a perpetrator following CSA. Included amongst these are: physical abuse; insecure attachment; parental substance abuse; and parental criminality (Nalavany et al. 2009; Simons et al. 2008). These and other factors are explored next. (Some of this research has already been referred to in Chapter 2, when the prevalence of CSA in sexual offending populations was explored).

Craissati et al. (2002: 229), found that a number of factors 'contributed to a predictive model' of sexual offending: namely, emotional abuse and/or physical neglect as a child; and 'having homosexual contacts in adulthood'. The childhoods of sexual offenders reporting CSA in Langevin et al.’s (1989) study, were marked out by: sexualisation relating to contact with adults, peers and also siblings; running away; dysfunctional family backgrounds, particularly relationships between father and son; parental abuse, and alcohol use; more frequent aggressive behaviour within the family; and parental criminality.

Others, too, have found a range of factors to be of significance: namely, again, a childhood marked by 'heightened sexuality' (Simons et al. 2008: 549; Simons 2002); exposure to pornography at an early age (before 10 years old) (Simons et al. 2008; Simons 2002); sexual activity with animals; masturbation from an early age (before 11); 'frequent exposure to violent media' during childhood; and 'insecure parental attachment bonds' (Simons et al. 2008: 549). Some researchers (Briere 1984; Connolly and Woollons 2008; Freeman-Longo 1986) have identified multiple episodes of victimisation by more than one abuser, and repeated abuse over time as risk factors in relation to adult sexual offending. Interestingly, however, most of those in the non-offender group in Briggs and Hawkins' study had experienced 'multiple abuse by multiple offenders' (p.225). Thus, the authors note that: 'sexual history frequency data are not sufficient to help us explain the transformation of victims into victimizers.' They further propose that it would be unsurprising if prisoners had 
not replicated the abuse they experienced, as they perceived such behaviour as normal and had been abused by many of those close to them whom they trusted. Indeed: 'the commission of sexual abuse on children was an accepted part of their world' (p.230). Briggs and Hawkins assert the point that: 'men who normalize their own experience of sexual abuse may be more likely than other victims to perpetuate the abuse.' (p.231)

Plummer and Cossins (2016), in a systematic review of existing evidence, identified four key factors which increase the likelihood that male victims of CSA will become perpetrators; namely, being abused at the age of 12 years or older; experiencing frequent sexual abuse; experiencing serious abuse; and having been abused by a perpetrator with whom the victim has a close or dependent relationship, for example, a father. The authors argue that the extent to which abuse at the age of 12 years or older represents a particular risk of subsequent offending, is grounded in the powerlessness (Cossins 2000) which young people experience during adolescence, in their relationships with adult males and male peers. Frequent and more serious CSA also engenders powerlessness; as does a close relationship with an abuser, due to issues relating to betrayal and trust. Plummer and Cossins (2016: 15) propose that: 'For male CSA victims, the development of a sexuality based on their abusive experiences may represent a cultural resource for expressing power and control'. Thus, a cycle of abuse may result, where abusive experiences are normalised and reproduced in the victimisation of others.

\section{Female-perpetrated CSA as a risk factor}

Some evidence has emerged that experiencing CSA by a female is a risk factor for subsequent perpetration of abuse in both adult and adolescent offenders (Duncan and Williams 1996; Glasser et al. 2001; Mathews et al. 1997; Salter et al. 2003). Glasser et al. (2001: 482), for example, found that out of a total of 24 males who had been abused by a female relative in childhood, 19 (79\%) became abusers. Indeed, 'the highest likelihood of a male victim becoming a perpetrator was associated with being abused by a sister or mother.' (p.488). The authors conclude that:

'abuse of males by female relatives may be more likely to contribute to the male victim becoming an abuser than abuse by male relatives or persons outside the family'. (Glasser et al. 2001: 487) 
Duncan and Williams (1996) explored the relationship between the gender of the perpetrator, and the use of force during abuse. Their sample was split into 3 groups: those reporting forced sexual contact with a male; forced sexual contact with a female; and sexual contact with a female which did not include the use of force. Significant differences were found between the 3 groups, with the group which had experienced forced sexual contact with a female most likely to sexually offend in adulthood.

Salter and colleagues (2003) followed up a total of 224 boys who had been sexually abused over a 7 to 19 year period. Twenty six (12\%), of those boys subsequently engaged in sexual offending, mostly against children, shortly after they had themselves experienced CSA. A number of characteristics set the offending population apart from the non-offending boys: namely, they were more likely to have been neglected; to have had poor parental supervision; to have observed severe episodes of violence within their own family; and to have been abused by a female.

\section{Children and young people as victims and victimisers}

Little attention was paid to 'juveniles' who sexually victimised others until the 1980s (Veneziano and Veneziano 2002: 247). Such behaviour was defined as 'normal experimentation or developmental curiousity'; and the emphasis was very much on adults who sexually offended. In recent decades, however, a growing body of evidence has emerged in relation to the population of children and young people who engage in SHB, and their prior experience of sexual victimisation. Hackett (2014: 1) draws attention to the fact that: 'in many cases, children and young people occupy dual identities as perpetrator of abuse and victim of harm'. The majority of these young people are adolescent males (ibid.); their victims are usually siblings, family members, friends or neighbours; and the abuse is characterised by age inappropriate sexual activity and coercion (Lovell 2002). Like adult sexual offenders they may: have poor social skills; be isolated from their peers; lack sexual knowledge; have low self-esteem; experience emotional loneliness; have poor educational attainment; and high levels of anxiety (Hackett 2014). Their backgrounds are often characterised by histories of sexual, physical and/ or emotional abuse (Veneziano and Veneziano 2002); and the abuse they perpetrate is thought to be a response to their own experience, and an attempt to regain control (Watkins and Bentovim 1992). Indeed, Bacon and Richardson (2000: 255) suggest that some children: 'try to integrate or master their 
experiences of traumatic victimisation by compulsively "playing them out”'. As part of this process, the child moves from 'a passive to an active role' - from powerless victim to powerful abuser - and in doing so experiences some 'relief'.

While it remains a hidden and somewhat taboo subject, a significant number of adult sexual offenders initially engage in SHB in adolescence (Edwards et al. 2012; Veneziano and Veneziano 2002; Veneziano et al. 2000). Skuse et al. (1997) point to 4 risk factors present in adolescent perpetration of CSA, regardless of whether perpetrators have themselves been victimised in childhood: experiencing physical violence; witnessing physical violence; the provision of intermittent care; and experiencing rejection by family. Others (Bentovim and Williams 1998: 104), too, have found that both witnessing and experiencing physical violence was a common feature in the backgrounds of abusers; in addition to 'discontinuity in care’.

Veneziano et al. (2000: 366) focused on the relationship between adolescent sex offender behaviors and victim characteristics with prior victimization, in a sample of 74 adolescent males engaged in 'residential treatment'. The majority (68; 92\%) had experienced CSA; and also demonstrated emotional, behaviour and psychological problems. It appears that for some adolescents in this sample, their behaviour was a 'reenactment of their own experience of sexual abuse' (Veneziano et al. 2000; 370). Indeed, they were 'more likely to select victims and sexual behaviors that were reflective' of that victimization' (ibid.: 372). Of note, is that those who had experienced the most severe form of abuse - anal penetration - were more likely to abuse others. It is hypothesised that, as this form of abuse is more traumatic, and victims may have experienced protracted abuse, it is more likely that the victim will subsequently abuse others.

Burton et al. (2002: 893) explored the childhood sexual victimisation of male, adolescent sexual offenders, comparing them with victimised, non-sexual offending male delinquents. The former: had closer relationships with their abusers; were more likely to have been abused by males over a longer period of time; and were more likely to have experienced a more 'forceful' form of victimisation, and to have been penetrated. Here, two factors best predicted sexual offending: abuse by both male and female perpetrators; and the level of force exercised during the abuse. 
Vizard et al. (2007: 69) identified a number of 'vulnerability factors' in their sample: including, a dysfunctional family environment; aggressive socialisation, where children are 'directly or indirectly exposed to models of aggressive behaviour' (p.70); inappropriate sexualisation; 'cognitive deficits', for example, a learning disability; and mental health problems such as self-harming. Moreover, Hackett et al. (2013) more recently found that two thirds of the young people in their study who had sexual convictions, had 'experienced at least one form of abuse or trauma' (p.237), including, for example, sexual or physical abuse; neglect; or domestic violence.

\section{The missing piece: Offenders' views of their childhood sexual victimization}

Simons et al. (2002: 1293) note that: 'From the research predictors of sexual offending, it appears that several factors can contribute to the development of a deviant sexual interest pattern.' Included amongst these are: childhood experience of sexual and/or physical abuse; and 'early exposure to sexually explicit material' (ibid). However, not all sexual offenders have experienced such abuse or were exposed to pornography from an early age. 'Thus, these factors appear to be neither necessary nor sufficient conditions for becoming a sexual offender.' (ibid.: 1294).

Much clinical and research energy continues to be expended on identifying those factors which may prove influential in the commission of sexual offences (Simons et al 2002). Yet, in spite of this attention, a plethora of 'unanswered questions' persist (Craissati et al. 2002: 225). Burton et al. (2002: 896), for example, note that little attention has been paid to: 'the relationship between the characteristics of sexual victimization and characteristics of sexual offending'. Thus, it remains 'an unexplored line of inquiry'. Moreover, Whitaker et al. (2008: 542) highlight the paucity of: 'work on the description and distinction among offender types, victim types, and situational aspects of child sex offending'.

Little emphasis has been placed on the distinction between those who offend against boys, as opposed to those who offend against girls; or on offending on an intra-familial as opposed to extra-familial level (Jespersen et al. 2009). Moreover, to date, there has been a tendency to 'lump all perpetrators together, irrespective of their experiences as a victim' (Glasser et al. 2001: 483). In this thesis, an attempt is made to address these gaps in the existing knowledge base by: exploring in detail the characteristics of 40 male child sexual abusers, including their 
childhood experiences and offending behaviour patterns. Data collected with regard to victims, including their age, gender and relationship to the abuser are also subject to detailed examination.

Childhood sexual victimisation is: 'one of the most frequently discussed events in explanations of adolescent and adult sexual offending' (Jespersen et al. 2009: 180). Yet, the perspectives of offenders on their childhood sexual victimization, are notably absent from the knowledge base. The emphasis has clearly been on establishing rates of CSA and exploring risk factors. While data on prevalence and risk are of significance, they fail to provide a detailed and individual account of sexual victimization; in that sense they are limited in terms of their contribution to future safeguarding and effective intervention. Indeed, in the midst of a battery of measures, the 'traumatized child is often lost' (Wilcox et al. 2004); and too many questions remain unanswered. What form did the offender's childhood sexual victimisation take? What age was the offender at the time they were abused (there is little data on this aspect of offenders' victimization (Jespersen et al. 2009))? What were offenders' perceptions of the abuse? Did offenders disclose the abuse they experienced in childhood, or attempt to do so? If so, what happened as a result? If not, why not? What was offenders' experience of intervention, if any? Who did they later abuse? What age and gender were their victims, and how, if at all, were they related to offenders? What attracted them to their victims? Did the abuse perpetrated replicate abusers' own childhood sexual victimization? These gaps in the evidence base warrant further attention; they may only be filled by a qualitative examination of this problem, characterised by the detailed narratives of offenders themselves. The research reported in this thesis aims to address such gaps in knowledge.

\section{Summary: Part 2}

In Part 2 of this Chapter, the emphasis has been on those factors which appear to place victims at increased risk of later perpetrating CSA. The risk factors highlighted included: inappropriate sexualisation; physical and domestic violence; exposure to pornography; female-perpetrated CSA; and insecure attachment. Part 2 concluded with some reflection on 'the missing piece', that is, offenders' perspectives on their childhood sexual victimisation and its aftermath. 
In this, and the previous Chapter, the focus has been on placing the problem of CSA in context, in order that the data collected from the 40 men who participated in this study may be better understood. The extent to which the findings of previous research on CSA including the risk factors highlighted here - are of relevance to those 40 men is explored during the Research Findings Chapters. In the next Chapter, the focus shifts to Methodology, and the research which lies at the heart of this thesis. 


\section{Chapter 4: Methodology (Part 1) Doing research with imprisoned child sexual abusers - The process}

\section{Introduction}

Here, the focus is on the methods employed in undertaking the research; and the rationale for adopting those methods. This Chapter is presented in 2 parts. In Part 1, the emphasis is on the process of doing research with child sexual abusers; and in Part 2, on the challenges inherent in such research. The Chapter is permeated by a sense of the personal: the process of self-reflection which persisted prior to, and during the fieldwork period and beyond, into data analysis and writing up findings; and the impact and 'cost' of doing research with this population.

Much of this chapter relates to the experience of undertaking research as part of the wider study referred to in the Introduction. As the research for the PhD was undertaken during the course of that wider study, it is not possible to distinguish between some of the challenges encountered. For, they apply to both the larger group of 101 offenders and also the sub-sample of 40 men who are the focus of this research. However, specific issues emerged as part of the process of asking offenders whether they had been sexually abused in childhood; these are considered - along with the wider methodological issues associated with doing research with this population - during the course of this Chapter.

Howitt (1995: 1) notes that being 'face to face' with a child sexual abuser:

'is not a comfortable situation. Most of us harbour a catalogue of beliefs and emotions about such people, which structures our perceptions of what to say, think and do'.

In doing research with abusers, those beliefs and emotions which are so personal, have first, to be acknowledged and then, managed. One learns a lot about oneself when doing research with this population; and one certainly needs to ask oneself some very searching questions at the outset, above all: do I have the capacity to listen to - and tolerate - 
explicit accounts of CSA on a daily basis, across a significant period of time? Moreover, do I want, or need, to listen to those accounts? Even though I clearly understood the benefits of listening to offenders, and the value of the research, these were recurring questions throughout the fieldwork for this study. They were questions I asked myself each day as I travelled to the prison to undertake interviews; and again when I left. Indeed, my final thought as I left the prison was often: how much more of this can I listen to? As Conte et al. (1989: 299) posit, no matter how often one is exposed to such explicit interview material, or to what extent one becomes de-sensitised to abusers' descriptions of the abuse they perpetrated: 'the impact of their words are not lessened'. This is because, at the heart of research 'conversations' with child sexual abusers lies: 'a deliberate process which inflicts pain and other consequences on young children' (ibid.). This is what proved most difficult to deal with.

Such internal conflict was compounded by two other factors: first, the sense in which I was unprepared for so many offenders to be such affable and ordinary men: fathers, grandfathers, and brothers. Yet, such ordinariness masked the severity of the abuse they had perpetrated, which had so often persisted across time. Second, because doing research with child sexual abusers, elicits such discomfort and unease in others, including those in academic circles. Indeed, as Howitt (pp.1-2) notes, it often: 'causes hardened research colleagues to shudder "how could you?" or to question the point of doing so'. One needs to be very sure of the value of engaging in such research to be in a position to defend it on both an internal and - in the face of such reactions - on an external level.

As already noted, the research reported here was undertaken alongside a larger study which examined the motivations and strategies of child sexual abusers. In this smallscale, qualitative, 'study within a study' (Williams 1995: 4), a phenomenological approach (Bryman 2008) was adopted, to provide a detailed account of the lived experiences (Garrett 2010) of 40 men who had been convicted of, and imprisoned for, a range of sexual offences against children. Much of the research on CSA focuses on adults' retrospective accounts of their childhood victimization (Finkelhor et al. 1997). In spite of the limitations of this approach - considered in some detail later in this chapter - 
which may include some distortion of memory and time (ibid.), this study is retrospective in design, and based on offenders' self-reports of CSA.

The approach adopted here is inductive; this allows: 'codes and themes to be derived from the content of the data themselves', with the aim of giving voice to offenders' experiences (Lambie and Johnston 2016: 902). Fondacaro et al. (1999) make the point that so often victims' perceptions of the abuse they experienced are overlooked in favour of the standardised measures and criteria used by professionals. Indeed, as noted in Chapter 3, the latter appear to take precedence over the 'reality' of victims' perspectives (ibid.: 368). As is the case in Durham's (2003: 311) research, which examined the experience and impact of CSA on a small sample of young men, the emphasis here is on a qualitative approach; and in particular, on: 'diversity and the uniqueness of individual experiences.' Durham (p.312) reminds us that individual experience is: 'subject to and created by interactions with others'. Moreover: 'Each level of interaction constitutes a site of learning and influence'. In the case of the men who are the subject of this research, interactions with others - those who abused them - resulted in a negative outcome. Of interest here, is the 'learning and influence' which characterized those relationships.

\section{The first task: reviewing the literature}

The aim, in undertaking the literature review, was to provide a starting point (Strauss and Corbin 1990), or 'framework for establishing the importance of the study' (Denney and Tewksbury 2013: 218). An extensive review of available literature on CSA and, in particular, of the links between victimization and offending, was undertaken at the outset. The review began with a search through the iFind Research system, using selected search terms related to the area of enquiry.

Sources identified included peer-reviewed journals, for example: Journal of Child Sexual Abuse; Sexual Abuse: A Journal of Research and Treatment; Child Abuse \& Neglect; Child Abuse Review; British Journal of Criminology; Criminal Justice and Behavior; Aggression and Violent Behavior; International Journal of Offender Therapy and 
Comparative Criminology. Other searches were undertaken of a range of relevant organizations, including, for example: NSPCC; Barnardo's; Child Exploitation \& Online Protection Centre (CEOP); Home Office; Ministry of Justice (MOJ); Office for National Statistics (ONS); and the National Offender Management Service (NOMS).

In undertaking the review, emphasis was placed on the direction which previous research had taken; and on establishing what was known and not known about the topic. The review enabled gaps in current knowledge to be identified; and informed the research questions for the study.

\section{Developing research questions}

'Empirical research is driven by research questions' (Punch 2005: 33). They are central to the research process for a number of reasons, they: have an organisational role, providing direction and a sense of coherence; emphasise the boundaries of the research; enable the researcher to remain focused on the task in hand; provide a structure which facilitates the writing up of the research; and provide an indication as to what data need to be collected and which methods should be employed (ibid.).

\section{The research questions: moving from the general to the specific}

In the first instance, the following 'general' (Punch 2005: 33) research questions provided direction for the study:

1.To what extent does childhood sexual victimization feature in the backgrounds of those who sexually offend against children?

2. What factors play a contributory part in the link between childhood sexual victimization and later sexual offending against children? 
A detailed review of the literature revealed substantive gaps in knowledge around the views of offenders' themselves on their sexual victimization; this led to the formulation of further literature-informed research questions which were designed to elicit difference between groups. These questions are listed in the Introduction.

\section{Quantitative or qualitative research?}

Much of the research on the topic of the links between victimisation and sexual offending against children is characterised by a quantitative approach. Of course, there are many benefits to this, not least the employment of large samples; and the use of statistical measures to analyse data (Punch 2005). A quantitative approach was not deemed appropriate for the wider study, as the aim was to elicit as much individual and detailed data as possible from offenders. However, the inclusion of closed-ended questions within the questionnaire designed for the study, allowed for the collection and analysis of a substantive amount of quantitative contextual data, relating to offenders' demographic details, for example. These data are drawn on during the course of the thesis.

Qualitative methods differ substantively from positivistic approaches, primarily because: 'the researcher's self plays a significant role in the production and interpretation' of data (Denscombe 2003: 268). Indeed, it is not possible for the researcher's beliefs, values and identity to be excluded from the research process. This issue is of particular relevance here and will be considered in further detail during the course of this chapter. As was the case in Dodsworth's (2014: 188) research on sexual exploitation, in this study: 'In-depth semi-structured interviews charted a chronological route through the participants' lives to consider experiences in their narratives'.

Qualitative research is often the subject of criticism due to small sample size which limits the extent to which findings may be generalised (Oaksford and Frude 2003). However, the aim of such research is not to formulate generalisable findings. Rather, it is to tease out individual perceptions of experiences (Coyle and Wright 1996). This research is characterised by offenders' retrospective accounts of childhood sexual victimisation and 
an interpretive approach to analysing those. On the 'interpretive tradition', (Gilbert 1993: 7) notes that it: 'seeks not so much explanations and predictions of social events as understanding what meaning and what significance the social world has for the people who live in it.' This sense of 'meaning' is a central feature of this study.

\section{Grounded theory}

This research is characterised by a grounded theory approach (Glaser and Strauss 1967): 'a research strategy whose purpose is to generate theory from data' (Punch 2005: 155). The emphasis is on social patterns (Glaser and Strauss 1967), and the following are implicit in the research design:

- Constant comparison - the creation of categories to explain data; and the reformulation of those categories as new forms of data emerge during the process of analysis

- Theoretical sampling - the employment of a purposive sample

- Systematic coding - the use of categories or codes to understand data

- Theoretical saturation - the point at which further interviews and data analysis serve no useful purpose in knowledge generation (Strauss and Corbin 1990)

- Multiple perspectives - the interpretation of the perspectives of research participants; an awareness of difference between groups, based, for example, on gender, class or race; and an awareness of one's own perspective, and the sense in which that might influence interpretation of data (op. cit.). 


\section{The questionnaire}

At the outset of the wider study, much emphasis was placed on designing a questionnaire that was characterised by clarity; where the questions were unambiguous and would be interpreted in the same way by all participants. This was particularly important for this population, who often have: difficulties with literacy; few qualifications; and poor social skills. Given that so much of the evidence on sexual offending provides a partial account only, the intention from the outset was that as much data as possible would be captured on these men. Questions 25(a) to 29(a) in Section 3 of the questionnaire were designed to elicit as much information as possible about offenders' experience of CSA. Of course, prior to interview, it was not possible to estimate how many of the men would report sexual victimization in childhood. The research findings on this topic are contradictory. Prevalence rates for CSA in sexual offending populations differ according to the method employed; the context within which the research is located; and the questions asked. In this research, much emphasis was placed on the language used in asking questions relating to childhood sexual victimisation. These were framed in such a way that offenders were given several opportunities to reflect on their childhood experiences in a range of contexts, thus allowing them the time and space to make their decision as to whether to report CSA.

Given the sensitive nature of this research and the need for a detailed approach, openended questions were included in the questionnaire alongside closed-ended questions. As was the case in Oaksford and Frude's (2003: 68) research on survivors' accounts of coping following CSA, open-ended questions allowed offenders to 'speak freely' about their own childhood sexual victimization and the impact of that on them. They also enabled the collection of data - in the form of 'rich narratives' (p.69) - which would not have been available had a purely quantitative approach been undertaken. The latter, on the other hand, allowed a considerable amount of demographic information to be collected about offenders; this enabled comparison between groups, for example, on the basis of age; marital status; educational and employment history; and offending behaviour. Moreover, the semi-structured questionnaire allowed offenders the opportunity to provide detailed responses where appropriate; and the researcher to probe 
and follow up on the information provided, to ensure that: stories which were 'individual' to the men emerged and that: 'the meanings ascribed to those experiences' were understood (Dodsworth 2014: 189).

In this study, the extent to which gender might play a part in inhibiting reporting could not be dismissed - that is, the extent to which some men might feel that to report experiencing CSA to a female researcher would, in some way, diminish their standing as males. Notwithstanding this, the majority of offenders were extremely open about their own childhoods and also their offending behavior during interview. Of course, there were some who preferred not to reflect on the former. In spite of this, data collected during often lengthy individual interviews, highlighted some issues not previously addressed in the literature on sexual offending; therein lies the strength and contribution of this research.

\section{Defining abuse}

One of the major challenges encountered by researchers in this field is the task of defining CSA in a way that is relevant to the population under study. At the outset of the wider study, much thought was given by the research team as to how CSA would be defined. Of course, all 101 men in that study had been convicted of, and imprisoned for, sexual offences against children; thus, they were aware of the 'meaning' of CSA in a legislative sense. However, at interview, several of the 40 men who are the subjects of this research, did not define the sexual activity they were exposed to in childhood as abusive, even though in definitional terms it clearly was. This was particularly the case where that activity involved other children and young people; and adult females. This is further explored during the course of the thesis. Definitional issues are considered here, as a means of placing in context such contrasting perspectives.

In the absence of one consistent definition of CSA (Brown et al. 2016), researchers have employed a range of questions when seeking to establish whether participants have experienced CSA. Afifi et al. (2009) for example, focused on inappropriate touching against the victims' will; and Kim et al. (2010) refer to having being touched in a sexual 
way as a child. Moreover, Alexander et al. (2000) and Testa et al. (2011) defined CSA as sexual contact which is unwanted. Both focus on age, albeit with differing emphasis. The former focus on the perpetrator being 5 years older than the victim and the use of threat or force is included; the latter, on contact abuse experienced before the age of 14 . In each, there are issues with specificity; what is meant, for example, by inappropriate touching; touched in a sexual way; and unwanted sexual contact? Each could be interpreted in any number of ways by those asking the question; and those being asked.

Thomas and Fremouw (2009) posit, that with so much research based on vague definitions of CSA, a lack of specificity results in the inability to draw: 'meaningful conclusions, compare results across studies, and replicate findings' (p.386). The solution, they suggest, is to employ 'a standard definition' (p.386) such as that used by Finkelhor (1984), where sexual victimisation is defined as sexual encounters involving children under 13 with persons at least 5 years older than themselves; and children of 13-16 years old with persons at least 10 years older. Sexual encounters are defined as: intercourse, oral-genital contact, fondling, or an encounter with an exhibitionist.

While age differentials are useful in determining abuse - and are now employed by many researchers - the prevailing issue, of course, is that abuse perpetrated by children and young people of a similar age to their victim may be excluded. Sexual abuse is often defined as sexual activity which 'occurs between adults and children' (Russell 1983: 133). Thus, sexually harmful behaviour by children and young people - who are peers or less than 5 years older than their victims - is often overlooked. In recognition of this, some researchers now place emphasis on a 2-5 year difference in age (Carlson et al. 2006). Even that can prove misleading, however, as the relationship between children of similar ages could also be coercive - for example, a child who is physically more mature might draw on that to their advantage.

Finkelhor (1994: 413) suggests, that in screening for CSA, the optimal approach is to: 
'ask multiple questions with very specific language about a variety of contexts in which abuse could have occurred, as opposed to a single screening question that asks about sexual abuse'.

In the research reported here, as was the case in Craissati et al.’s (2002: 229) study, the task of eliciting the prevalence of CSA in offenders' backgrounds was approached 'from a number of angles' (ibid.). Thus, CSA was defined as sexual activity - either contact or non-contact - before the age of 16 with an individual at least 5 years older than the child (Finkelhor 1984). During interview, all the men who participated in this research were asked whether they had been sexually abused in childhood based on this definition. However, they were also asked a number of other questions which were designed to incorporate any abusive or negative experiences in childhood, both within and outside the family; and also to enable a focus on abusive or negative experiences with children and young people. This was undertaken as a means of eliciting as much information as possible about offenders' childhoods; and establishing to what extent, if any, they perceived a link between their childhood experiences and their later sexual offending. Those questions were:

- To what extent do you see a link between your own family experiences as a child and your offences against children?

- To what extent do you see a link between your relationship with childhood friends, inside and outside school, and your offences against children?

- To what extent do you see a link between your relationship with any significant adult(s) outside your immediate childhood family and your offences against children?

The adoption of this approach resulted in the collection of data which might not otherwise have been forthcoming. For, while some men might have been reluctant to respond to a question which asked them directly about their experience of CSA, they did 
respond to the questions above; and in doing so, they sometimes referred to their own childhood sexual victimisation.

\section{Gaining access}

Access is key to any empirical research study (Bryman 2008). When the research involves imprisoned child sexual abusers, it can prove even more problematic. However, fortuitously, two members of the research team for the wider study had previously undertaken research with a small number (7) of male child sexual abusers within a prison establishment in the UK (see Colton and Vanstone, 1996; 1998). This usefully facilitated further access to the same establishment.

In the early stages of the wider study, a series of meetings were held with the prison governor and lead psychologist. During those meetings, which all 3 members of the research team attended, they were informed of: the nature of the research; its aims and objectives; and the proposed structure and format of the questionnaire. Following this, access to the prison population was granted; and - as I was to undertake all the interviews for the wider study - arrangements were put in place for my induction into the establishment and its working practices; in particular, safety and security measures. In the first instance, this included an individual security check. Further discussion on issues related to this process may be found in Part 2 of this Chapter.

\section{The research sample}

A total of 101 men were interviewed for the wider study over a one year period. In line with a grounded theory approach, the sample size for that study was not pre-determined. Rather, it was decided as the research progressed, and interviews continued until theoretical saturation (Strauss and Corbin 1990) was reached: at 101. The 40 men who are the focus of this research were included in that sample of $101 \mathrm{men}$. The prison database, which included information on offences committed, was used as a sampling frame. It usefully provided a complete and current list of prisoners; and was therefore 'an 
objective list of the population' from which participants could be selected (Denscombe 2003: 17). Purposive sampling methods were employed (Bryman 2008: 333) as: 'such sampling is essentially strategic and entails an attempt to establish a good correspondence between research questions and sampling'.

All those who had been imprisoned for sexual offences against children were included in an initial list of participants. Eighty-three offenders were excluded from the list because they did not fit the criteria for selection: 40 denied their offences, and 43 had been imprisoned for fraud, deception or sexual offences against adults. Prisoners were selected for interview according to their release dates; those whose release was imminent were interviewed first. Prison staff ensured that an updated version of the prison database was made available to me on a weekly basis. This enabled new prisoners to be included in the list of possible participants on arrival at the establishment.

The database included information on the men's offending; and also included details of their victims. It therefore, proved a useful aid in triangulating data (Bryman 2008). Following interview, the men's accounts of their offending were cross-checked with those contained in the data-base; all details in the former were consistent with those held on the database. This was unsurprising, given that the men presumably assumed that I had access to information about them; therefore there would have been no gain in misleading me at interview.

\section{The impact of the Sexual Offender Treatment Programme (SOTP) on the sample}

Fifteen out of 40 of the men in this sample had either completed the SOTP, or were undertaking it at the time of interview. The SOTP was established in the UK in 1991, as part of the prison service strategy for sexual offenders. It is a cognitive-behavioural model of treatment which has proved influential on a policy level (Friendship et al. 2003). It focuses on social and psychological explanations of offending (McGuire 1995), with an emphasis on group-based delivery (Doel and Sawdon 1999). Amongst the elements of the Programme delivered to offenders are those which focus on attitudes and 
beliefs; victim empathy; and relapse prevention (Hudson 2003). It is not the intention to provide an exhaustive account of the SOTP in this thesis, rather, the aim in highlighting it here, is to point out its relevance to some of the men who participated in this study: in particular, the possible impact on their narratives of participation in the Programme. This issue is revisited in later sections of the thesis, in particular, the Research Findings Chapters.

\section{Contacting participants}

With the task of questionnaire design complete, attention shifted to deciding how best to contact participants and introduce the research to them. Prison staff helpfully assisted with this. A covering letter including information on the research aims and objectives was distributed to potential participants, in staged phases, on a week by week basis, through staff on the prison wings (see Appendix for a copy of the letter). A total of 155 prisoners were contacted in this way, and their participation in the research requested. A considerable amount of time was spent with prisoners on the wings following distribution of the letter. This investment of time enabled responding to queries from prisoners about: the purpose of the research; the extent to which confidentiality and anonymity could be assured; how and where the data would be held; who would have access to it; and how data would be used. Understandably, offenders were extremely concerned about each of these issues; and the process of clarification, and reassurance, on each count, proved central to the success of the study. This is considered in further detail later in this Chapter.

A total of 54 men out of the 155 contacted, refused to be interviewed. Several expressed concern, in spite of much reassurance, that information elicited at interview might be passed on to the media. Indeed, some believed that I was a journalist. Others were undertaking the Sex Offender Treatment Programme (SOTP) and felt unable to participate as they were in the process of confronting their offending. A third group did not believe that the research would benefit them in any way. They explained that they had admitted their offending; were 'doing their time'; and did not wish to talk about their 
offences any further. Those who participated in the research did not differ, with regard to characteristics or offence profiles, from those who decided not to take part.

\section{Ethical issues}

\section{Gaining ethical approval}

Ethical approval for the wider study was granted by the Centre for Criminal Justice and Criminology Ethics Committee at Swansea University.

\section{Ethical issues in undertaking research with prisoners who have been convicted of CSA}

Ethical issues abound in undertaking research with prisoners who have been convicted of CSA and these are considered next. Above all, when collecting and analysing data, and disseminating findings, researchers are expected to refrain from questionable practices (Robson 2002) such as:

- Involving participants without their knowledge or consent

- Exposing them to harm

- Using coercion

- Withholding information about the research and deceiving participants

- Not treating participants fairly or with respect

It is further expected that they abide by 'data protection principles' as laid out in the Data Protection Act (1998) (GOV.UK 2014). In particular, researchers are expected to ensure that the data they collect are:

- Used fairly and lawfully

- Used for limited, specifically stated purposes

- Used in a way that is adequate, relevant and not excessive

- Accurate 
- Kept for no longer than is absolutely necessary

- Handled according to people's data protection rights

- Kept safe and secure

- Not transferred outside the UK without adequate protection

Of course, when undertaking research in a prison environment, there are additional challenges relating to that specific environment. These include handling data supplied by the establishment with particular care, given its nature; and within prison boundaries only. In this study, information relating to offenders was accessible only within the prison and was not to be removed under any circumstances.

Researchers are also required to adhere to relevant codes of conduct, in this case, those of the British Society of Criminology. The Society’s most recent Statement of Ethics (2015: 2) highlights the 'general principle' that:

'researchers should ensure that research is undertaken to the highest possible methodological standard and the highest quality in order that maximum possible knowledge and benefits accrue to society’

In terms of participants, the emphasis is upon minimizing harm and stress resulting from the research; ensuring their dignity and autonomy is preserved; protecting their rights and privacy; ensuring that they are fully informed about the research and that their participation is voluntary and based on informed consent principles; and assuring confidentiality, while making clear to participants the limitations of that assurance. Some of these issues - for example, confidentiality and anonymity - warrant further consideration, given the nature of the population in this research study.

Confidentiality and anonymity: the issues as they relate to a prison sample

Confidentiality and anonymity are central features of empirical research (Bryman 2008). However, when participants are imprisoned child sexual abusers, limitations to confidentiality take on a particular dimension. As already noted, a considerable amount of time was spent prior to each interview discussing confidentiality - and, of course, its 
limitations - and anonymity. While all those who participated in this research were offered confidentiality and anonymity, they were also informed of the limitations to that confidentiality. Should information be disclosed at interview that might result in harm to an individual; or in relation to additional offences, then, it was made clear from the outset that information would be passed on to prison staff.

Offenders were also informed that while the research findings would be widely disseminated, and their verbatim responses included in a range of publications arising from the research, their identity would not be disclosed. Rather, they would be allocated a study identification number; and a pseudonym, which would serve to anonymise their contribution to the research. A further complication arises, however, when offenders are asked specifically about their offending. During interviews, many of the men provided a considerable amount of personal details about their victims, including their names and where they lived. This information, unless carefully managed, has the potential to cause serious harm and distress to both victims and their families. Given this, much care has been taken in the handling of data to ensure that details which might identify victims have either been changed beyond recognition, or omitted.

\section{Informed consent}

All participants were fully informed of the nature of the research at the outset. In line with the protocol for informed consent, they were provided with information on:

- The identity of the researcher

- The purpose/aims of the research

- Expectations about participants’ contributions

- The right to withdraw consent 
- Confidentiality/anonymity and security of data (Denscombe 2003)

All were asked to sign a consent form indicating that they had been fully informed about the research; and that they were prepared for their interview to be taped. The consent form contained the signature of the participant and was dated; it was also signed and dated by the researcher (see Appendix for copy of covering letter and consent form).

\section{The interviews}

With one exception, offenders were interviewed in a designated room located in the prison psychology department; one prisoner's mobility problems resulted in his interview being undertaken in his prison cell. The semi-structured questionnaire was administered on an individual basis to all participants. The use of pilot interviews assists with issues of clarity and understanding in relation to the questions to be posed (Dodsworth 2014). Thus, the first 12 of the 101 interviews undertaken for the wider study were used for piloting purposes, with detailed notes being made of responses. In terms of this study, 3 out of 40 interviews were part of that piloting process. No amendments were made to the questionnaire following piloting.

Prisoners' consent to audio tape interviews was sought in the remaining 89 cases; only 3 men refused, due to concerns as to who might have access to the audio tape. In these cases, detailed notes were again taken of responses. In total, 86 interviews were taped and fully transcribed. The tapes were held securely at the University; and in line with data protection principles, identifying information was held separately to the tapes.

Interviews lasted between one and three hours. Prisoners were available for interview during a specific three-hour period each morning and afternoon. These timescales had to be strictly adhered to, and prisoners had to be collected from, and returned to the wing in accordance with them. Failure to have adhered to this requirement would have seriously compromised security, and the daily regime of the establishment; and thus the viability of the research. As a result of these timing constraints, however, several interviews ended abruptly and had to 
be resumed the following day. In those cases, a number of challenges were encountered, including the need to deal with the frustration experienced at the interruption to the 'flow' of the interview, particularly where some time had been spent in encouraging prisoners who at first were reluctant to talk, to respond; and the need to 'reset' the interview context, reestablish a sense of trust, and re-create a ‘safe' space in which offenders could participate.

\section{Asking the question about CSA}

When asking whether or not offenders had been sexually abused in childhood, adopting a sensitive, and careful, approach was crucial. The research requirement to elicit specific information had to be balanced with an awareness of the unpredictable nature of participants' response to the question and the need to manage that (see Roberts 2011 for further discussion on this). While some of the men provided a detailed response to the question of previous sexual victimization, others - like Hugh, for example, who had been abused by both male and female perpetrators - were more reticent. The need for a careful and cautious - approach was brought sharply into focus during the interview with another man: Gareth. At first, he refused to consent to a taped interview. However, following reassurance about confidentiality and anonymity, he later consented. He was at first extremely reluctant to talk about his childhood; and initial attempts to establish whether he had been sexually victimized as a child were unsuccessful. Gareth’s manner was aggressive and confrontational from the outset of the interview; some time was therefore spent in reminding him that he was under no obligation to respond to any of the questions; and in making clear - once more - the purpose of the research and my role as interviewer, that is, to listen to his account without challenging him about, or judging him on, his offending. Some time later, reassured, Gareth began to talk about his childhood. This issue is considered in further detail in Part 2.

The impact on all 101 men of participating in the research was given much thought prior to the commencement of interviews, as it was expected that some would reflect on emotive issues relating to their childhood and sexual victimisation. Opening up the personal to public gaze has the capacity to impact negatively on research participants 
(Durham 2003). Thus, all those who were interviewed were advised to contact prison staff if they required support following interview. I was not made aware of any issues associated with the interview process by prison staff; and assume that the support mechanisms available to prisoners, met their needs in this regard.

\section{Data analysis: Qualitative data}

As a consequence of the open-ended nature of the questions posed to offenders during interview - and in line with a grounded theory approach - a considerable amount of qualitative data were collected during the study. These data were managed using constant comparison of themes (Strauss and Corbin 1990). Interviews were fully transcribed, and the computer package WINMAX aided thematic analysis and coding. Data analysis involved 3 stages: open coding; axial coding; and selective coding (Strauss 1987). Of course, notwithstanding efforts to ensure rigour, this process is subjective in nature (Oaksford and Frude 2003), and the resulting limitations cannot be ignored. At the outset, open coding allowed for the identification of broad themes in the interview transcripts. More detailed axial coding enabled key themes to be identified. Finally, selective coding focused attention on those codes of most relevance to the study.

During interviews with the 40 men who participated in his study, it became clear that a number of consistent themes were emerging from their narratives: this enabled further probing during subsequent interviews (Strauss and Corbin 1990). The key concepts (ibid.) which emerged from data analysis related to offenders' experiences and perceptions of CSA; its impact on them; and the extent to which CSA contributed to later offending.

The following models/theories informed analysis of specific aspects of the data set. All retain relevance today. They are summarised in the following Chapter, as a point of reference for the research findings:

- Summit's (1983) Child Sexual Abuse Accommodation Syndrome - drawn on in relation to the impact of childhood sexual victimisation; and responses to it 
- Spiegel's (2003) Sexual Abuse of Males (SAM) model of dynamics and effects of CSA - drawn on for its specific focus on male victims; and in relation to the impact of childhood sexual victimisation; and victims' response to it

- Finkelhor and Browne’s (1985) Traumagenic Dynamics Model - drawn on in relation to the impact of CSA

- Finkelhor's (1984) Four Pre-Conditions Model - drawn on in relation to the concept of becoming an abuser: 'the making of a child molester'

- Ward and Keenan's (1999) Core Implicit Theories - drawn on in relation to the 'thinking' and belief systems which support offending behaviour

The men's accounts in the Research Findings Chapters, are ordered according to the 3 main themes below:

- Offenders' experiences of CSA

- Offenders' perceptions of CSA

- Becoming an abuser

\section{Data analysis: Quantitative data}

A substantive amount of quantitative data were collected during the course of the research. The questionnaire was coded at the outset to allow for quantitative data analysis. Data relating to the wider study - and also the study reported in this thesis - were analysed by means of the Statistical Package for the Social Sciences (SPSS); the latter by means of SPSS (Statistics Version 22).

Forty out of a total of 101 cases were of relevance to this research; data relating to these men were extracted from the wider data set. They were then analysed according to the following groupings: 
- 40 men who reported CSA

- 32 men who reported male-perpetrated CSA only

- 5 men who reported female-perpetrated CSA only

- 3 men who reported male and female-perpetrated CSA

The process of analysis began with the 40 men who reported CSA. Distinctions were then made within and between 3 groups of offenders - as indicated above - in order to examine difference where that might exist. As the sample was small, exploratory analysis was undertaken; descriptive statistics were elicited; and frequency tables produced. These data are drawn on during the course of the Research Findings Chapters; in some cases they are presented in table form for ease of viewing.

\section{Validity of data}

Research on CSA is fraught with challenges and characterized by methodological limitations (Oaksford and Frude 2003). The validity of data obtained through retrospective reports of CSA has proved a contentious issue (Briere and Conte 1993; Widom 1989); this warrants some reflection here. First, with the passing of time, information may be lost or distorted (Burton et al. 2002; Dodsworth 2014; Oaksford and Frude 2003; O’Leary 2009). Second, the issue of social desirability might feature (Weeks and Widom 1998) and respondents might deny that they have been sexually abused due to a number of factors including: embarrassment; wanting to forget the past or protect parents or caregivers; and a belief that they deserved the abuse. Of course, as already noted, male victims are less likely to disclose abuse and seek help so it is unlikely that research will capture the full picture in terms of male victimization.

Further concerns about the validity of interview data are brought even more sharply into focus when respondents are sexual offenders. Although research evidence indicates that adults who have experienced sexual victimisation in childhood are not likely to exaggerate their experience or its impact (Edwards et al. 2001), there appears to be some reluctance to believe that the same is true of sexual offenders. As already noted - and as 
evident in other research studies (Gannon et al., 2008) - confidential information about offences held at the prison establishment was cross-checked with information provided by offenders at interview. In each case, participants provided a consistent account of their offending.

Some argue that because childhood sexual victimisation is accepted as an explanation for sexual offending (Hansen and Slater 1993), it is possible that abusers 'learn to provide such accounts during therapy’ (Worling 1995). Others assert that offenders are more likely to over-report such experiences with the aim of moderating responsibility for their offending; or positively influencing parole boards (Weeks and Widom 1998). However, this is not supported by the research evidence. Indeed, it appears that under-reporting is more likely to occur (Widom and Morris 1997). Within the general male population, evidence suggests that males are more likely to under-report CSA due to issues relating to masculinity, and what it means to be a male; if they are abused by males, they are even less likely to report as they may have concerns about their sexuality, and fear that they may be perceived as homosexuals by others (Hansen and Slater 1988). It is likely that offenders share these concerns; and it appears that some deny their sexual victimization in childhood due to feelings of shame and embarrassment (Dhawan and Marshall 1996).

In the research reported here - as was the case in Craissati et al.’s (2002: 234) study offenders did not appear to over-report childhood victimization or 'fabricate' accounts. On the contrary, some of the men were reticent to report CSA and only did so some time after they were asked the question about childhood sexual victimization. Having avoided responding initially, they returned to the subject of their own victimization when they felt comfortable doing so. Moreover, when they did respond, there was a consistency to their accounts, which were often detailed; and reflected: 'different degrees of severity and trauma' (ibid.). 


\section{Summary: Part 1}

In Part 1 of this Chapter, the emphasis has been on methodology: namely, the process of doing research with child sexual abusers; and the rationale for adopting a method characterised by a qualitative, phenomenological approach. The Chapter opened with some reflection on the literature review process, and the development of research questions. This was followed by discussion around the merits of adopting a qualitative as opposed to quantitative approach to the area of enquiry; and the deployment of grounded theory techniques. The challenges encountered in designing a questionnaire which would capture a detailed account of offenders' childhood sexual victimisation were highlighted; and issues related to gaining access to participants in a prison environment considered.

Ethical issues abound in doing research with sexual offenders; and these were explored in some detail: in particular, those relating to confidentiality, anonymity and informed consent. The interview process was outlined; and issues encountered in asking the question about childhood sexual victimisation were discussed. Part 1 ended with an emphasis on data analysis and validity. In Part 2 of this Chapter, attention shifts to the challenges encountered in undertaking research with child sexual abusers in a prison environment. 


\section{Methodology (Part 2) Doing research with imprisoned child sexual abusers - The challenges}

\section{Introduction}

Here, attention shifts to the challenges encountered in undertaking research with child sexual abusers in a prison environment. The first thing to note is that this is very much a personal - and reflective - account of those challenges and my response to them. Undertaking this research required the development of a distinctive skill-set; and an understanding of self that I have not encountered in any other research I have been involved in. These are issues that are given scant attention in the research methods literature; as such, they are considered in some detail here.

Doing research in prisons presents a number of challenges unique to that setting. Included amongst these are the difficulties associated with gaining access to offenders; their physical environment; and, of course, security issues. In undertaking interviews with imprisoned child sexual abusers, these difficulties are further compounded by: the sensitive and emotive nature of CSA; and the often explicit detail provided about offending (Petrillo 2007).

Much research has been undertaken with sexual offenders in the prison environment (King 2000). The focus has often been on risk management (Ward 2014); and issues relating to assessment and treatment (Abracen and Looman 2005; Frost 2004). However, increasing attention has focused on the impact of working with sex offenders (see, for example, Amen 2002; Crabtree 2005; Petry 2005; Shelby et al. 2001). Some attention has also been paid to the gender issues which emerge when working with sex offenders (Cowburn 1998; Genders and Player 1995; Petrillo 2007). Notwithstanding this, there are: 'too few detailed discussions on the doing of research in prisons' (King 2000: 309), with scant attention paid to the process of undertaking research with imprisoned male child sexual abusers, and the part played by gender in that process. Here, an attempt is made to address this deficiency in the research knowledge base. 


\section{Undertaking research with prisoners who have been convicted of CSA: the challenges}

In overcoming the challenges encountered in doing research with prisoners who have been convicted of CSA, some of King's (2000: 297-308) 'ten nostrums for field research in prisons' prove helpful: that you have to be there to understand the environment; you have to do your time in order to establish a role; you should not work alone unless you have to - especially pertinent for researchers focusing on CSA, where a supportive network outside the prison is crucial; you have to know why you are there, and be in a position to fully inform staff and prisoners of the research aims and objectives; you must always remember that research has costs for staff and prisoners and that the research exercise should cause as little disruption as possible to the daily life of the establishment; you must know when to open your mouth and when to keep it closed, maintaining an awareness that 'prisons are dangerous places and it would be foolish not to monitor what one gives out'(p.304); you must do whatever you have to do to observe but do not go native, that is, ensure that boundaries are maintained between the researcher, prison staff and prisoners; and finally, you should triangulate data where possible.

What form do these challenges take? First, of course, is the overarching need to take account of security and personal safety issues during the fieldwork period. Moreover, access, the physical location of respondents, and the limitations of confidentiality all feature. Documents that are available on-site only, together with restricted daily access to prisoners serve to compound the research task. As does the fact that the needs of the establishment outweigh those of the researcher. Thus, a flexible approach in the face of a limited timescale within which to undertake the fieldwork is crucial, as one can often find oneself in a situation of having to reschedule interviews unexpectedly.

On a personal level, previous experience of research within a prison environment proved invaluable. Notwithstanding this, a considerable amount of energy was required in the face of competing needs: to quickly become accustomed to the daily routine of the establishment; to 'fit in' and remain as unobtrusive as possible, causing little disruption to the daily life of the prison; and to remain focused all the while on the research task. In the 
initial stages of the research, spending time being there to understand the environment; and doing your time in order to establish a role (King 2000) was essential. As already noted, security issues were central to the research, and an intensive induction period was undertaken at the outset. Having successfully navigated that, I was issued with my own set of keys. This did not sit comfortably with me because it impacted so visibly on identity: was I an independent researcher or one of the prison staff? Yet, I was aware that without accepting that responsibility, the research could not have been undertaken. Given their other commitments, it would have proved impossible for staff to assist with bringing prisoners to the designated interview room, and returning them to the wings following interview.

\section{The issue of trust}

Colton and Vanstone (1998: 516) draw attention to the 'highly secretive' nature of child sexual abusers; and the extent to which their: 'thinking, strategies and abuse itself are [always] hidden within the abuser's world'. Engaging them in the research process requires much effort. Waldram (2007: 963), argues that where research participants are imprisoned sexual offenders: 'the establishment of trust in the research relationship is essential to creating safe places' in order that stories can be told. Those stories involve the sharing of intensely personal and sensitive information. This sharing process represents a risk for all those who open up their lives to others (Mearns and Thorne 2008) whether as part of therapeutic intervention, or as research participants. For sexual offenders, that risk is amplified because the information they disclose is sometimes shameful (Ward et al. 2006); and because they may already have been vilified and shamed as a result of their offending (Knight and Modi 2014).

The process of establishing trust, with both staff and prisoners, proved time consuming. However, it was an essential precursor to data collection. Both were concerned about security issues, albeit for different reasons. Staff concerns centred on the physical security of prisoners within the establishment, and the potential for a researcher to seriously compromise that. Prisoners were concerned that information imparted at 
interview would not remain secure and confidential, and may be used against them, perhaps in a media campaign. Such concerns were allayed by frequent assurances about the handling and management of data. Regular communication with staff was absolutely vital; as was the understanding and acknowledgement that maintaining a secure environment was crucial, and the responsibility of all those working with offenders, including researchers. Thus, all prison staff were fully informed about the study at the outset. During the course of the project, appropriate members of staff were informed in advance of the schedule for interviews: for security purposes, and in order to minimise disruption.

Considerable time and effort was spent on the prison wings in the initial stages of the research introducing the study to prisoners. The research aims of further protecting children and preventing abuse were made explicit at the outset (and reinforced throughout); and assurances were given with regard to confidentiality, while of course, also making clear the limitations of that and the potential for information to be passed on if necessary. It is expected that researchers will ensure that their participants are not harmed during the research process (BSC 2015). Indeed, assurances given about confidentiality and anonymity serve as a means of reinforcing their safety to some extent. However, when the participants are convicted and imprisoned sexual offenders, there are a number of 'ethical tensions' (Cowburn 2005: 49) which emerge, not least with regards to the limits of confidentiality. For, in such cases, the concept of harm stretches beyond the participant - and the research process - to undiscovered victims who may have been harmed in the past; and forward to those who may remain at risk from offenders who have not been detected. While research in this field aims to obtain as much data as possible about offenders and their offending behaviour, during the course of an interview, information may be forthcoming about previous harm and ongoing risk. Thus, tensions are evident between the search for 'knowledge' and the need to respond ethically in order to protect others from harm. Implicit here, is the requirement to manage 'the boundary between confidentiality and public protection' (ibid.: 49). 
The BSC Statement of Ethics (2015) sets out the duty placed on researchers to act on information received about the abuse or neglect of a child. It draws attention to the fact that, in the UK, researchers 'have no special legal protection that requires them to uphold confidentiality', as in the case of lawyers and medical professionals. Indeed, they and the data they collect 'can be subject to subpoena where they may have evidence relating to a case' (ibid.: 11). This, it is argued, should be carefully considered by researchers when confidentiality is assured; and efforts should be made from the outset of the research to make clear the limits of confidentiality to participants, as opposed to offering 'absolute confidentiality'. In the research reported in this thesis - in line with BSC standards - it was made clear to offenders from the outset that while every effort would be made to ensure confidentiality and anonymity, there were limits to those assurances. Indeed, the boundaries to confidentiality were made explicit: namely, that information would be passed on to prison staff if, at interview, participants disclosed offences that had previously not been detected. Moreover, if information was provided about children who were currently at risk of CSA, the same protocol would be adopted.

None of the 40 men who participated in this research study disclosed offences which had previously not been detected. This is perhaps unsurprising, given the conversations which took place with participants around confidentiality limits prior to interview. Considering ongoing risk: many of those who had sexually abused participants in childhood had passed away by the time of interview; several men did not disclose the identity of their abuser; some provided very little detail of their abuse; and some had disclosed to prison staff. In some other cases, abusers had been identified and convicted of their offences. One man made clear that having previously not disclosed abuse by his mother, he had no appetite now - some years later - to report that abuse to the relevant authorities, given the likely consequences of doing so. The extent to which this might represent a risk to others was, of course, a concern. However, such concern has to be weighed against the likelihood that, unless participants are comfortable with those consequences, a situation may arise where 'second-hand information' is passed on, which may later be denied; and where there is resistance to co-operate with any future investigation (Cowburn 2005: 60). 
Feminists draw attention to the place of power within the research process, and the hierarchical relationship evident between researcher and respondent (Punch 2005). Indeed, Oakley (1981) has placed much emphasis on the need for a reciprocal exchange of information with research participants in order to address these issues and to achieve optimum results from individual interviews. Within this research, however, there was no room for such reciprocity; and much care was taken to ensure that no personal information was divulged to prisoners. Indeed, while attempting to build trust I remained alert to the risks of resorting to the personal, maintaining an awareness that 'prisons are dangerous places' (King 2000: 304). Emphasis was placed on the fact that I was not employed by the prison service, or any other criminal justice agency, rather, that I was independent and had a research role within a university setting. While the potential for the power dynamics evident within the research process to impact on data collection was acknowledged, this was accompanied on another level by an understanding that 'prison is about power relations’ (Waldram 2007: 964). Placing myself outside the system in which those power relations function was crucial, in order that the research role was not compromised. Maintaining clear boundaries between myself, prison staff and prisoners, and not 'going native' (King 2000) enabled this placing outside, and setting apart from others within the establishment.

\section{Asking questions: listening to responses}

Interviews allow us to access individual perceptions and understanding of situations, and are 'one of the most powerful ways we have of understanding others' (Punch 2005: 168). Kvale’s (1996) qualification criteria for interviewers prove a useful starting point when reflecting on the characteristics of a successful interview. Kvale proposes that an interviewer should: be knowledgeable with regard to the focus of their interview; provide structure and purpose to the interview; use questions which are clear and jargon-free; be gentle and tolerant of pauses, allowing participants an opportunity to think and respond; demonstrate sensitivity through attentive listening and an empathetic approach; be open and flexible in terms of response; steer the interview along an appropriate path in order that the research questions are answered; be critical, in the sense that inconsistencies in 
responses are identified and followed up; remember what is reported in order that connections can be made throughout; and interpret responses without prescribing meaning. Bryman (2008) adds two further criteria to this list: interviewers should demonstrate a balanced approach by neither talking too much nor too little; and also an awareness of the ethical issues which are integral to the research process, including confidentiality.

While the above prove helpful, additional skills are required when undertaking individual interviews with child sexual abusers. In the first instance, as Marshall et al. (2000) note, telling their story can prove extremely difficult for sexual offenders. For, those stories often involve the recounting of abusive experiences in childhood: experiences which may result in feelings of fear, shame and humiliation. Moreover, many offenders: 'are unable to conceptualise, let alone articulate, their stories, both of their life experiences and their current offending' (Knight and Modi 2014: 133). Thus, much effort is required to encourage offenders to 'open up' and reflect on their life histories, including their offending. The extent to which 'emotional literacy' (Knight and Modi 2014) might feature here warrants some attention. Emotional literacy is a skill associated with probation practice (ibid.). However, it also appears to be of some relevance to the task of undertaking social science research with sexual offenders, due to its focus on:

'knowledge of our own emotions and the ability to recognise and respond empathically to the emotions of others. It includes an awareness of the causes, triggers and expression of emotions in ourselves and in others, and requires an underpinning value base of respect, positive regard and a non-judgemental approach towards offenders' (ibid.: 194)

Understanding of self (Vanstone 2007), or self-awareness, is central to emotional literacy. Knowing myself and understanding my emotional threshold was crucial in undertaking this research. While the telling of their story proves difficult for sexual offenders, listening to those stories also proves difficult. Knight and Modi (2014: 143) note that probation officers who work with sexual offenders: 'need to be comfortable with their own sexuality and in talking about sexual matters. They need to be able to hear accounts of sexually explicit behaviour'. This also applies to those who undertake research with 
sexual offenders. My first task lay in acknowledging my perspective on CSA, and accepting that, for me, it is a heinous crime which has a significant impact on victims. However, if I was to achieve the research aims, and collect my data I would need to present a non-judgemental attitude to abusers. Had I not engaged in a period of reflection - prior to undertaking the fieldwork - in which I reached an understanding about how that could be achieved; and also the nature of my 'relationship’ with offenders in my sample, I doubt whether those men would have so readily told their stories. I too, had to 'open up' to some extent; not in terms of communicating with offenders about my personal life and who I was, in an attempt to develop rapport: that would have been foolhardy. Rather, I needed to re-consider my perspective on CSA; reflect on my beliefs and value base; and reach a compromise which allowed me to connect with offenders and 'recognise and respond' to their emotions (ibid.). That connection was achieved through frank and honest discussion with the men about the research objectives; my interest in safeguarding and prevention; and through a consistent interviewing approach, where offenders were not confronted about their responses, but were listened to.

That listening process represented one of the key challenges encountered in interviewing child sexual abusers. The act of self-regulation (Howe 2008) - controlling one's emotions - enabled some detachment. Notwithstanding this, one cannot discount the 'narrative risk' (Waldram 2007: 966) involved in hearing the stories of sexual offenders; the details of the abuse they have sometimes experienced in childhood, and the abuse they perpetrated as adults; and the potential for exposure to such stories to 'haunt, rattle, and challenge one's belief in a moral world and the inherent goodness of humankind.' (ibid.: 966). The task of disconnecting from accounts detailing the nature of offending required considerable energy; and retaining control of the interview when confronted with such detail proved extremely difficult, requiring a form of disciplined empathy (Medlicott 2004).

Knight and Modi (2014: 134) argue that emotional literacy enables an individual to demonstrate to offenders that they have the capacity to 'hear and contain the sometimes very powerful and disturbing aspects' of their narratives. This is an issue which has 
received scant attention in the research methods literature and warrants further examination. For, the sense in which a researcher is emotionally resilient (ibid.) and recognises the potential impact of offenders' discourse on them - and the research process - and devises a strategy for dealing with both is crucial to the outcome of a study.

Many of the questions asked of offenders at interview were extremely sensitive. Amongst the most difficult to ask was the question about childhood sexual victimisation. For many of the men, the discomfort they experienced at hearing that question and formulating their response was tangible. In some cases, as the men described their own CSA, their vulnerability was evident (Knight and Modi 2014). That vulnerability took various forms: in some cases, the pain of abuse - both physically and psychologically - came through in the discourse. In other cases, offenders' self-report of sexual victimisation was couched in a more aggressive response as they demonstrated anger at their abuser/s. Other men were clearly anxious not to portray themselves as victims and in these cases there was some sense of bravado evident. In the face of such diversity, the over-riding question for me was: how should I respond? These men were sexual offenders but they were also victims - a status which does not sit comfortably with attempts to make sense of their offending. Remaining task-focused assisted with the process of maintaining some sense of detachment. However, it was impossible to remain completely detached during this aspect of their narrative. One could not help but be affected by the men's accounts of the sexual abuse they experienced in childhood. Indeed, these data have had the most profound effect on me as a researcher, as I have attempted to reach an understanding of offenders' status as both victim and victimiser. In many cases, their accounts of their childhood sexual victimisation were extremely detailed, providing a graphic illustration of the highly sexualised nature of their childhoods. No matter how well practiced one is in the art of detachment, such accounts have the capacity to pervade one's personal space at the point at which they are first heard; and also to persist across time.

Remaining alert to the potential for the questions asked to elicit a hostile response was vital. Indeed, throughout each interview, it was essential that a heightened awareness of environment, respondent and the nature of the questions was maintained. All respondents 
were asked whether they had experienced CSA. Of course, one cannot ignore the concerns expressed by many with regard to offenders' self-reporting: namely, that abusers might report childhood abuse and link that to their behaviour in order to explain their offending, minimise their guilt, access therapy, or evoke a sympathetic response which might work to their advantage on sentencing (Craissati et al. 2002; Glasser et al. 2001; Simons et al. 2002). Yet, others (Weeks and Widom 1998: 358) argue that underreporting of CSA is more likely, and that:

'incarcerated individuals may fear the appearance of vulnerability within the prison setting more than any potential benefit they might receive by admitting to childhood victimisation experiences.'

None of the men in the wider study were offered incentives to participate in the research, and it did not appear that any of the 40 who disclosed CSA, did so in order to elicit sympathy or favour. Interestingly, as already noted, in some cases disclosure occurred during the course of the interview - at a point where the offender felt 'safe' - as opposed to in direct response to specific questions about childhood experiences.

\section{The impact of gender}

As already noted, the overwhelming majority of individuals imprisoned in the UK are male. While many of the psychology and probation staff who work with sex offenders are now female, prison culture is dominated by a 'hegemonic form of masculinity' which is 'heterosexual, misogynistic and violent' (Cowburn 1998: 236). With much CSA perpetrated by males, and victims female (Finkelhor and Korbin 1988), it proved impossible to ignore the duality of my position: as a researcher and a woman. Those who perpetrate sexual crimes often perceive themselves as dominant in relation to women (Connell and Messerschmidt 2005). Gender issues proved central to the research experience reported here and represented a considerable challenge. In considering these, Petrillo's (2007) work on the extent to which gender features in female probation officers' work with high risk offenders provides a useful reference point. 
The need to avoid colluding; manage inhibitions and feelings of revulsion; and deal with attempts to intimidate (Petrillo 2007) is crucial when undertaking interviews with child sexual abusers. Developing a strategy for overcoming 'feelings of revulsion or anger about the offences'; and dealing with 'techniques of manipulation and control employed by the offenders' (Petrillo 2007: 397) was an essential prerequisite to interview. Indeed, as one of Petrillo's probation officers noted:

'[you] have to not allow themselves to be visibly shocked in their [sexual offenders'] presence... You have to be just consciously aware all the time that you're a woman...' (p.399-400).

These were issues I was keenly aware of during the research. Like the officers in Petrillo's study I found the 'very explicit sexual language' and graphic detail about offences extremely difficult to listen to. Often, such language was used as a 'shock' tactic, to test out my response, and the extent to which I could be 'controlled'. At times, I sensed that attempts were being made to intimidate me and I, too, was very aware that displaying signs of being uncomfortable, would demonstrate vulnerability. Retaining objectivity and control of the interview in the face of that, proved a challenge which took considerable effort to overcome. A number of things enabled me to remain 'unharmed' by the interviews. First, having a supportive network of experienced researchers at the University; and the opportunity to de-brief with them; second, a timetable for the fieldwork which allowed time away from the prison; third, the devising of a personal strategy to consign accounts of abuse to the prison environment, until they were revisited, for the purposes of data analysis, in a 'safe' place: research base.

\section{Summary: Part 2}

In the first part of this Chapter, the focus was on the process of doing research with imprisoned child sexual abusers. In Part 2, the focus has been on the challenges encountered in undertaking research with that population. King (2000: 285) refers to 'the craft of doing research in prisons', and some of King's (pp.297-308) 'ten nostrums for 
field research in prisons' have been drawn on in an attempt to tease out the issues which emerge in interviewing those who have perpetrated CSA.

The issue of trust was explored, alongside the concept of emotional literacy and the 'need to be able to hear accounts of sexually explicit behaviour' (Knight and Modi 2014: 143); and the impact of gender on the interviewing process. Doing research with imprisoned child sexual abusers is a research voyage unlike any other, in which a range of methodological and personal challenges present. How might these be overcome? By understanding the prison environment, and establishing a role within it; never feeling isolated due to the presence of a supportive network back at research 'base'; knowing why you're there; and above all, always maintaining boundaries between both staff and prisoners (King 2000).

Waldram (2007: 963) questions whether there are: 'some people whose stories ought not to be heard.' There is a paucity of research on the views of child sexual abusers (Colton et al. 2009). Yet, their stories - unpalatable to many - provide a 'unique' source of data (Elliott et al. 1995: 591). Listening to those stories can impact negatively on researchers. However, if we are to work towards protecting children; preventing abuse; and more effective intervention with offenders, we have a responsibility to make every attempt to listen: 'however difficult that may be’ (Waldram 2007: 969). 


\section{Chapter 5: Theoretical underpinnings to the research}

\section{Introduction}

There has been much emphasis on theoretical, causal explanations of sexual offending (Marshall and Marshall 2002; Starzyk and Marshall 2003). Yet, the issue of the link between victimisation and offending, and how some victims become abusers, is fraught with contradiction and conflicting evidence. In this thesis, offenders' narratives are drawn on in an attempt to understand their 'lived experiences' (Garrett 2010: 680), in particular, their childhood sexual victimisation; their perceptions of that; its impact on them; and the extent to which it contributed to their becoming an abuser. There is a dearth of research on the 'experiences of childhood for child sexual abusers' (ibid.); and on the complexity of those experiences as described by offenders themselves. It is hoped that the narratives of the 40 men who participated in this research will shed at least some light on that complexity.

\section{Understanding sexual offending - the theoretical context}

In this Chapter, the emphasis is on the theoretical context to sexual offending against children. It is not intended that an exhaustive account of all existing theories are provided here. Rather, the merits and limitations of some of those theories are considered at the outset. Then, the focus shifts to those theories which were found to be of particular relevance to this thesis, and which have been drawn on in the analysis of offenders' narratives. In the section which follows, the emphasis first, is on Marshall and Barbaree's (1990) integrated theory; and then on Ward and Siegert's (2002) Pathways Model.

\section{Marshall and Barbaree's (1990) integrated theory}

Marshall and Barbaree's (1990) integrated theory and Finkelhor's (1984) multi-factor analysis continue to underpin much work with sexual offenders. (Finkelhor's preconditions model is considered later in this Chapter as one of the theories of relevance to the data collected for this study). Marshall and Barbaree's (1990) multi-factorial theory 
focuses on sexual offending generally and the developmental, onset and maintenance factors associated with both rape and CSA. Here, 'early development is of critical importance in creating offence-related vulnerabilities' (Ward et al. 2006: 34). One of the central features of this theory is its emphasis on the need for adolescent males to learn to differentiate between aggression and sexually driven impulses; and to control such aggression during sexual activity. It is proposed that achieving such an understanding is compounded where individuals demonstrate insecure attachment; poor self-esteem, and interpersonal skills; and an inadequate coping style. While some men demonstrate resilience and will not offend - due to the presence of characteristics including a set of values, beliefs and attitudes which enable resistance to sexual offending - in others, there is evidence of vulnerability to offend. Such vulnerability takes the form of adverse developmental experiences; biological influences, including hormonal changes during puberty; and prevailing attitudes and cultural norms about sexual activity and gender roles.

Marshall and Barbaree posit that puberty is a time when sexual preferences are formed and sexual scripts acquired. Individuals who are unable to cope with the biological and hormonal changes and accompanying sexual feelings which emerge at this point, are more likely to meet their needs through deviant sexual behaviour. In summary, a sexual offence results from the interaction of vulnerability with situational factors such as rejection or the breakdown of a relationship. Key here, are factors including individual vulnerability stemming from adverse childhood experiences; the biological and social processes associated with puberty; their interaction with disinhibitors such as substance abuse; victim availability; and the opportunity to offend. Whereas masturbation may have been used to manage negative states in adolescence, this later progresses to sexual offending which is maintained through the use of sex as a coping mechanism; and cognitive distortions (CDs) which function to justify sexually abusive behaviour. Adolescents' inability to distinguish between sexual and aggressive forces may result in their coalescence, and the propensity to demonstrate aggression in sexual situations. The tendency towards abuse is influenced by attitudes to children, norms of sexual behaviour, the availability of child abuse images, and the degree of societal tolerance to abuse 
(Lanyon 1991). Many will deliberately create opportunities to offend; others may react to situations or stimuli, for example, stress, anger, or alcohol (ibid.).

Lanyon (1991: 40) refers to Marshall and Barbaree's (1990) work as: 'perhaps the most comprehensive attempt to present a global theory of deviant sexual behaviour'. Ward et al. (2006: 39) too, extol Marshall and Barbaree's work, highlighting its emphasis on early development and attachment issues, and the work remains of relevance. Indeed, the emphasis on individual deficiencies as causal factors in sexual offending has proved influential in the evolution of therapeutic intervention which focuses on addressing intimacy deficits in offenders (Marshall 1999). However, there are a number of limitations to the work and these warrant some attention. In the first instance, the theory purports to explain all sexual offending; this, of course, is problematic given the diverse nature of offending and offenders. Moreover, its emphasis on those who begin offending as adolescents is also problematic, for it fails to explain offending which begins in adulthood; as is its focus solely on sexual offending which involves aggression.

\section{Ward and Siegert's (2002) Pathways Model}

This model is multi-factorial in nature and builds on Marshall and Barbaree's integrated theory, and Finkelhor's precondition model. The proposal here is that there are a number of causal factors or pathways that result in CSA. Each pathway comprises a range of components which engender problems commonly found in adult child sexual abusers, and play a contributory part in offending. These problems include: difficulties in emotional regulation; loneliness and social isolation resulting from poor social skills and intimacy deficits; cognitive distortions; and sexual deviance characterised by fantasy and arousal, or distortions in sexual scripts. There are a number of strengths associated with this model, including its recognition of the diverse nature of the sexual offending population; the reasons for offending; and the extent to which individuals may vary with regard to the nature and severity of presenting problems. Under the Pathways model, such distinctions may be located in traits such as vulnerability, with situational triggers interacting with individual traits to result in CSA (Ward et al. 2006). The emphasis is on 
'a number of distinct, and interacting' (ibid.: 74) Pathways to the perpetration of CSA, each of which comprises social, cultural and psychological factors. The model is, however, limited on an explanatory level to adult sexual offending: it does not provide an explanation of SHB by children and young people (ibid.).

The 5 Pathways leading to the commission of CSA are:

1. Multiple dysfunctional mechanisms - here, distorted ideas about child sexuality prove influential, as does deviant sexual arousal and distortions in sexual scripts.

2. Deviant sexual scripts - these are characterised by perceptions of relationships as purely sexual, sometimes as a result of sexual violence in childhood, and the inappropriate sexualisation which results. Sex is perceived as a physical act, removed from any sense of the personal: from emotion or intimacy. Those who follow this Pathway use others as mechanisms for achieving sexual gratification.

3. Intimacy deficits - here, there is evidence of insecure attachment resulting in difficulties with adult relationships. The loneliness which ensues is a factor in the substitution of a child as a partner, as opposed to another adult. There is a sense here in which there is emotional safety in the use of children as sexual partners, given that they will more readily be accepting of the offender.

4. Emotional dysregulation - Ward and Siegert propose that two forms of emotional dysfunction are evident in sexual offenders: first, those who have problems with anger management may abuse a child as a means of punishing their partner, or because they lack control. Second, at times of stress, and in the absence of support mechanisms, some might use sex as an emotional coping strategy (Cortoni and Marshall 2001), including sexual activity with children.

5. Antisocial cognitions - sexual offenders who follow this Pathway are distinguished by attitudes which are supportive of offending behaviour generally. They are defined as 
generalists as opposed to specialists, and it is likely that they will have committed a range of offences aside from sexual offences. Here, antisocial attitudes result in the discounting of norms or taboos which prohibit sexual activity with children, and there is an emphasis on meeting individual sexual need. There is also often evidence of patriarchal attitudes and a sense of superiority in relation to others.

There is some resonance between some aspects of the Pathways model and the data collected for this study, for example, the extent to which offenders might exhibit difficulties with adult relationships; may abuse a child as a means of punishment; use sex as a coping strategy; and demonstrate patriarchal attitudes. However, its emphasis is on adult sexual offending; clinical phenomena (Ward et al. 2006); and 'dysfunctional psychological mechanisms' or processes (Ward and Beech 2006: 60). Moreover, its focus is on the Pathways which lead to the perpetration of CSA; it does not explain why such abuse might persist (ibid.). Given this, it does not represent the best 'fit' with the research reported in this thesis.

Having reflected on some of the explanations put forward for sexual offending, attention now shifts to those theories/models which were found to be most relevant to this research study, and which, it was felt, would offer the optimum opportunity to explore the data collected and interpret the findings in as meaningful a way as possible.

\section{Understanding offenders' experiences and offending behaviour}

During interviews with offenders, a number of consistent themes emerged from their narratives, relating to: the experience of being sexually abused in childhood; their perceptions of that; its impact; and the extent to which, in their view, CSA contributed to later offending. It became clear with each interview undertaken, that a number of models/theories were of relevance to the men's accounts; and that their application to the data collected would assist in understanding their experiences. This was reinforced when the process of data analysis began and further connections were made. Thus, the justification for drawing on the models/theories listed below during analysis and 
presentation of findings, is grounded in their 'fit' with the men's experiences. Of course, each have their limitations - and these are recognised below - however, taken together, they have enabled the data to be explored with reference to frameworks which were found to be of greatest relevance to the topic of enquiry.

\section{The models/theories of relevance to this study}

Three of the models/theories listed below focus on the impact of CSA for victims; and two on the perpetration of CSA. The aim, in drawing on these models/theories in conjunction with others was to provide as comprehensive an account as possible of the victimisation and offending of the 40 men who participated in this study.

- Summit’s (1983) Child Sexual Abuse Accommodation Syndrome - drawn on in relation to the impact of CSA on victims and their response to it

- Spiegel's (2003) Sexual Abuse of Males (SAM) model of dynamics and effects of CSA - drawn on for its specific focus on male victims; and in relation to the impact of CSA; and victims' response to it

- Finkelhor and Browne’s (1985) Traumagenic Dynamics Model - drawn on in relation to the impact of CSA

- Finkelhor's (1984) Four Pre-Conditions Model - drawn on in relation to the concept of becoming an abuser: 'the making of a child molester'

- Ward and Keenan's (1999) Core Implicit Theories - drawn on in relation to the 'thinking' and belief systems which support offending behaviour

Each of these models/theories is summarised below. (A more detailed account of their content may be found in the original sources. It is intended that the summaries serve as a reference point for the data presented in the Research Findings Chapters). At this stage, it 
is also important to note that feminist explanations of sexual offending are also of some relevance to the research reported here. However, they are restricted by the extent to which they can be applied to male victims and female perpetrators. Therefore, while their contribution to the field of sexual offending is acknowledged, and they are discussed later in this Chapter - and drawn on to some extent during the course of the thesis - their applicability to the data collected is limited.

\section{Understanding the impact of CSA}

\section{The Child Sexual Abuse Accommodation Syndrome (Summit 1983)}

Summit (1983: 177) describes the Child Sexual Abuse Accommodation Syndrome as: 'a simple and logical model' which aims to:

'improve understanding and acceptance of the child's position in the complex and controversial dynamics of sexual victimization.'

As is so often the case in much research on CSA, the Syndrome's focus is on 'the most typical female victim' (p.180). Nevertheless, there is some explicit reference to male victims, particularly with regard to the category 'accommodation'; and each aspect of the Syndrome is applicable to males - and therefore of relevance to this study - who, it is argued are more likely to experience 'greater isolation' and exclusion if they do tell. This, according to Summit is based on the fact that:

'While there is some public capacity to believe that girls may be helpless victims of sexual abuse, there is almost universal repudiation of the boy victim' (p.180).

The Syndrome focuses on children's reactions to abuse and comprises 5 categories:

- Secrecy

- Helplessness

- Entrapment and Accommodation

- Delayed, Conflicted and Unconvincing disclosure 


\section{- Retraction}

During the course of this thesis, the extent to which each of the categories listed above feature in offenders' narratives is explored in some detail. As might be expected in sexual offending populations - given their diversity - each of the categories are of relevance to varying degrees. Secrecy, for example, was an over-arching feature of many of the men's experience of childhood sexual victimisation, with many holding the secret of that victimisation through childhood to adulthood. Many of the men had experienced multiple episodes of abuse which persisted, and the sense of helplessness and entrapment emanating from that was palpable in many of their accounts. Moreover, accommodation also featured, particularly in those who had been abused while in institutional care. Disclosure too, was a central feature of the men's narratives, in the sense that it was often delayed, and responses were inappropriate. In the section which follows, each of Summit’s 5 categories are summarised for contextual purposes.

Secrecy: Summit notes that whichever form CSA takes, the secrecy associated with it: 'makes it clear to the child that this is something bad and dangerous' (p.181). Indeed, the 'pervasive secrecy' (p.181) which accompanies the abuse results in the stigmatization of the victim who will be fearful of the outcome; and will experience a 'sense of badness and danger'.

Helplessness: On this, Summit notes that children are subordinate and helpless 'within authoritarian relationships' (p.182). There is an expectation that they will obey adults; and as most abuse is perpetrated by those known or related to the victim, the 'imbalance of power' is increased, further reinforcing 'the helplessness of the child' (p.183). Wherever abuse happens, and in whatever context, the victim therefore has: 'no choice but to submit quietly and to keep the secret’ (p.183).

Entrapment and Accommodation: CSA is not usually perpetrated on one occasion only. If the child does not seek out or receive protection immediately the abuse begins, there 'is no further option to stop the abuse' (p.184). Indeed: 
'the only healthy option left for the child is to learn to accept the situation and survive. There is no way out, no place to run. The healthy, normal, emotionally resilient child will learn to accommodate to the reality of continuing sexual abuse'.

Accommodation is evident not only in terms of the abuse experienced, but also in relation to an awareness of betrayal by someone who may be in a caring role and ostensibly should be a protector. If the 'outrage' at the abuse is not resolved, the child will experience an increasing sense of helplessness and rage. In females, this manifests itself in self-hate; self-harm; promiscuity; running away; suicidality; substance abuse; and in some cases, in later years, harmful behaviour towards their own children. In male victims, the sense of rage is likely to turn: 'outward in aggressive and antisocial behaviour' (p.185). Substance abuse; depression; violence; CSA; and rape: 'seem to be part of the legacy of rage endowed in the sexually abused boy'. (p.185). All these 'accommodation mechanisms... are part of the survival skills of the child' (p.186).

Delayed, Conflicted and Unconvincing Disclosure: Most abuse is never disclosed. Disclosure usually results from conflict with the family; accidental discovery of the abuse; or an increased knowledge on the part of the victim as to the nature of appropriate sexual activity. Family conflict may serve as a trigger to disclosure. This usually occurs some years after the abuse began and when there is: 'an eventual breakdown of accommodation mechanisms' (p.186). This often occurs during adolescence as the child victim seeks to develop a more independent life; and begins to challenge parental authority. Summit notes that such victims seek 'understanding and intervention' at the very point they are ‘least likely to find them’ (p186). Adults and professionals find the child's behaviour - and anger - challenging and are more likely to: 'identify with the problems of the parents in trying to cope with a rebellious teenager' than with the victim's plight. They fail to understand why the victim did not complain at the point at which the abuse began; and assume that the claim of alleged abuse is a fabrication in response to some form of control or punishment. 
Retraction: On this, Summit notes that: 'Whatever a child says about sexual abuse, she is likely to reverse it' (p.188). This results from the guilt experienced at the destruction of family relationships; and the desire to: 'preserve the family'. Thus: 'The children learn not to complain. The adults learn not to listen.'

While, as already noted, Summit's model is limited by its emphasis on the female victim, its strength lies in its description of the: 'pattern and sequence of interaction among the victim, the abuser, and the potential caretakers' (Leonard 1996: 108). The Model also usefully sheds some light on the apparent 'contradictory behaviour' of victims during the process of disclosure (ibid.).

\section{The Sexual Abuse of Males (SAM) Model (Spiegel 2003)}

Unlike Summit, Spiegel focuses specifically on males - and this model is therefore of particular relevance to this study - and on the dynamics and effects of CSA. It is important to note that there are some parallels here with Summit's work. However, Spiegel's (2003) work is, of course, more recent than Summit's. Amongst its strengths are, of course, its focus on males as victims; and its emphasis on the social construction of victimisation. However, it pays little attention to the effect of victims becoming victimisers. In this thesis, Spiegel's SAM Model is drawn in conjunction with Summit's (1983) model, as a means of further understanding victims' experience of abuse, in relation to some of the categories listed below: namely, concealment; invalidation; reconciliation; and compensation.

The Model comprises 7 categories which focus on: 'precipitating events, abusive episodes, their aftermath, and the sociocultural context in which these events occur' (p.137). The categories included in this Model are:

- Subjection

- Sexual abuse

- Concealment 
- Invalidation

- Reconciliation

- Compensation

- The cycle continues

Subjection: Here, a boy is prepared for CSA by 'subtle or blatant interactions' which result in 'boundary diffusion and role confusion'. This can sometimes involve grooming. As a consequence of the way in which sexual offenders: 'distort reality, impose roles, and lie to boys about love, safety, and trust', boundaries become dispersed and roles confused.

Sexual abuse: CSA involves progression from non-contact to contact activity. In some instances, offenders move from using sexualised language; to touching during the course of play; to more invasive touch; and then perhaps to penetration. However, recognition is paid to the fact that some cases of abuse are not characterised by progression and are more 'acute' in nature.

Concealment: This relates to the 'pervasive secrecy' inherent in CSA, which functions on both a personal level and in a social sense. It is argued that the abuse is: 'typically enforced by the perpetrator, reinforced by social mythology, and complied with by the boy' (p.167). Spiegel (2003: 175) argues that the 'sociocultural disregard for sexually abused boys' functions to inhibit disclosure. This is based on assumptions, or myths that:

- Boys are not sexually abused

- If they are, it happened because it was something they wanted

- If abused by a male, the victim must be gay

- If abused by a female, it was not really abuse

- Boys who are sexually abused are not able to take care of themselves (Spiegel 1995) 
Spiegel further notes, that for male victims of CSA: 'enforcers of concealment reach far beyond the sexually abusive relationship' (p.180). They are grounded in misunderstanding and an emphasis on the male perpetrator/female victim model of CSA; stereotypical images of gender - of males as aggressors and females as typifying passivity; and cultural misconceptions of motherhood and disbelief that mothers - and other females - can be abusers. All serve to 'create barriers around the sexually abusive relationship' (p.180), reinforcing a male victim's sense of responsibility for his own abuse and prohibiting telling.

Invalidation: Here, the emphasis is on the distortion of reality through victim 'defense strategies', including numbing and denial. Young males aspire to develop a number of traits that define them as masculine: namely, independence; power; dominance; aggression; and courage. Those who experience CSA, however, lose power; become dependent; are fearful; submissive; and subservient. For a male, the latter represents: 'the antithesis of all he is striving to be' (p.181).

In the face of the socially constructed argument that males are not victims, those males who do experience CSA move - in an attempt to survive and to preserve some safety and stability in their lives - towards accepting and believing abuse myths and doubting their own experiences. Thus, the distortion of reality through concealing, minimizing and denying abuse becomes the norm. As do feelings of stigmatisation resulting from the abuse. For some victims, numbing takes the form of alcohol or drug misuse.

Reconciliation: This refers to the process whereby the victim submits to the sexual abuse and takes responsibility for it in order to survive. There appear to be some parallels here with Summit’s (1983) category: ‘accommodation’.

Compensation: This includes victims' attempts to counteract or neutralize the 'internal conflict' resulting from CSA, through the restructuring of events. In order to undo the effects of CSA, and of 'feeling flawed' (p.242), and to distance themselves from it, some 
victims engage in compensatory activity. This may include behaviour associated with overtly male traits, such as aggression.

The cycle continues: CSA persists until the point of disclosure; the authentication of the victim's account of abuse; and the child is protected from further victimisation.

\section{The Traumagenic Dynamics Model (Finkelhor and Browne 1985)}

Finkelhor and Browne’s (1985) Traumagenic Dynamics Model was developed to aid understanding of the way in which CSA impacts on victims. Again, the focus is predominantly on the female victim, however, with the factors which generate trauma 'based on the subjective reactions of the female child' (Spiegel 2003: 22). Nevertheless, the Model is also applicable to male victims, and is of particular relevance to the narratives of the men who participated in this research study.

The Model relates impact to 4 dynamics:

- Traumatic sexualisation

- Betrayal

- Stigmatisation

- Powerlessness

Each of these were found to be of relevance - to differing degrees - to offenders' accounts of the impact of the CSA they experienced.

\section{Traumatic sexualisation}

This 'refers to the conditions in sexual abuse under which a child's sexuality is shaped in developmentally inappropriate and interpersonally dysfunctional ways' (p.355). The following ‘distinct processes combine to contribute to traumatic sexualisation': 
1. the rewarding of victims: 'for sexual behaviour that is inappropriate to their level of development'

2. due to the rewards, victims 'learn to use sexual behaviour...as a strategy for manipulating others to get their needs met'

3. due to the attention received from abusers, parts of victims' 'anatomy become fetishized and given distorted importance and meaning'

4. offenders' behaviour leads to confusion and 'outright misconceptions about sexual behaviour and sexual morality’ on the part of victims

5. 'a child's sexuality can become traumatized when frightening and unpleasant memories become associated in the child's mind with sexual activity' (p.355-356)

\section{Betrayal:}

Here: 'children discover that someone on whom they were vitally dependent has caused them or wishes to cause them harm' (p.356). Betrayal is evident to some extent in most cases of abuse and functions in differing ways. The child might experience betrayal at the point they are first abused when someone they trusted causes them harm. Alternatively, betrayal might occur later when victims realize they were lied to and manipulated into doing something 'bad’ (p.356).

Of interest, is Finkelhor's (1987: 356) point that:

'The dynamic of betrayal in sexual abuse encompasses not only the child's experience with the offender but also with non-offending family members'.

Indeed, for many victims, the sense of betrayal is greatest when they discover that mothers or others within their social network disbelieve them and fail to protect them.

\section{Stigmatization:}

Here, the focus is on: 'the negative messages about the self - evilness, worthlessness, shamefulness, guilt - that are communicated to the child around the experience' (p.357). Such messages emanate from abusers who blame the victim, and also from others' 
negative attitudes to victims who are often labeled 'spoiled goods' in the case of female victims; and 'queer' when victims are male. Indeed, it appears that: 'Because so many negative attributions exist concerning molested children, it is hard for sexually abused children to escape the dynamic of stigma' (p.357).

\section{Powerlessness:}

There are 2 elements inherent in powerlessness: '(1) a child's will, wishes, and sense of efficacy are repeatedly overruled and frustrated and (2) a child experiences the threat of injury or annihilation' (p.357). Finkelhor argues that many features of sexual victimization can result in a feeling of powerlessness. However:

'the most basic form of powerlessness, and one central to sexual abuse itself, is the experience of having one's body space repeatedly invaded against one's wishes’ (p.358).

This, it is argued: 'is a unique element to the sexual abuse trauma'.

The second aspect of powerlessness results from the coercion and violence which some victims experience. Children can also experience powerlessness after the abuse has ended 'when they find themselves unable to control the decisions of the adult world' (p.358), during the course of an investigation.

Finkelhor notes that the impact of CSA can be located within one or two of the traumagenic dynamics. Traumatic sexualisation, for example, has been noted in the behaviour of young victims who often demonstrate sexual knowledge and behaviour beyond their years. Several of the other effects evident in victims may be associated with the betrayal they experience, for example, grief; depression; extreme dependency; and susceptibility to re-victimisation. Some (see, for example, Boney-McCoy and Finkelhor 1995: 1415) argue that perceptions of oneself as 'spoiled goods' following sexual victimisation in childhood might: 'create vulnerability for later sexual abuse’. 
For some, the stigmatization experienced results in a sense of isolation, self-harming and suicidality. The feeling of powerlessness victims experience can result in a range of problems including: anxiety and fear; running away; difficulties with schooling and later with employment; and depression. Of particular relevance to this thesis, is the argument that for some victims, particularly males, CSA results in a need to dominate or control; and sexually abusive behaviour. The latter is seen to stem from 'the desire to be powerful and even fearsome to compensate for past powerlessness' (p.362).

Having outlined those models/theories of relevance to the impact of CSA on victims, attention now shifts to the two models/theories which were drawn on in relation to the concept of becoming an abuser: 'the making of a child molester' (Finkelhor 1984); and the 'thinking' and belief systems which are supportive of offending behaviour (Ward and Keenan 1999).

\section{Understanding sexual offending}

\section{Finkelhor's Four Pre-Conditions Model: 'the making of a child molester'(1984)}

Finkelhor's (1984: 53-54) Four Preconditions Model of Sexual Abuse is one of the most often cited theories of CSA, and represents the 'first multifactorial explanation' of this form of offending (Ward et al. 2006: 19). It has remained influential in a clinical sense, and its use is evident in a range of professional settings including probation, and social work (Ward and Hudson 2001). Its appeal lies in its ordered linking of causal factors to elements of the offence process (ibid.). Indeed, Ward and Hudson (2001: 291) argue that it provides a 'clear framework' for studying males who have sexually abused children. Others (Ward et al. 2006: 20), too, applaud its recognition of the complexity of CSA and the contribution of a range of factors in its perpetration, namely:

'the psychological needs and motives of the offender, situational and contextual variables, parenting practices, and social attitudes toward children and sex’. 
The model was developed as a means of building on the existing theoretical knowledge on sexual offending against children, which Finkelhor argues had previously ignored 'sociological factors'. It draws on data relating to offenders, victims and their families, and represents a combination of explanations, both psychological and sociological. It includes 4 factors which, when combined, may lead to the perpetration of CSA: emotional congruence or attachment to children; sexual arousal to children; blockage from normal sexual relationships; and disinhibition. These 4 factors, it is argued, contribute to the: 'making of a child molester' (Finkelhor 1984: 37). The first 3 explain the development of a sexual interest in children; the fourth, 'why this interest manifests as sexual deviance' (Ward and Beech (2006: 58).

Finkelhor presents these factors in the form of questions:

1. 'Why does a person find relating sexually to a child emotionally gratifying and congruent?

2. Why is a person capable of being sexually aroused by a child?

3. Why is a person blocked in efforts to obtain sexual and emotional gratification from more normatively approved sources?

4. Why is a person not deterred by conventional social inhibitions from having sexual relationships with a child?'

These factors are perceived as 'complementary processes', each of which may contribute to the 'making of a particular abuser'. Under the Model, before CSA can occur, 4 preconditions need to be met:

1. There should be motivation to sexually abuse a child

2. Internal inhibitors to offending need to be overcome

3. External inhibitors to offending need to be overcome

4. The child's resistance needs to be 'undermined' or overcome 
In terms of motivation - pre-condition I - 3 elements are present. These are: emotional congruence - where emotional needs are satisfied through sexually relating to a child; sexual arousal - where the child is a source of sexual gratification; and blockage - here, alternative sources of sexual gratification are either not available or are found to be less satisfying. It is not necessarily the case that each of these elements has to be present in order for abuse to be perpetrated; although often components of each are evident. An offender may abuse a child, for example, without experiencing sexual arousal. Rather, the abuse may be more a means of satisfying an emotional need. Moreover, 'blockage' may not necessarily result in abuse - an offender may abuse a child 'for the sake of variety' while other sources of sexual gratification are also available. Finkelhor (1984: 36) argues that sexual abuse: 'needs to be seen within a social and cultural framework' (1984: 5657); and locates explanations of sexual offending within individual and social-cultural levels. Several of the individual and social/cultural levels of explanation prove pertinent to the offenders in this research and are considered during the course of the thesis.

Emotional congruence is defined as the 'fit' between the adult's emotional needs and the child's characteristics' (p.38). On an individual level, emotional congruence can be explained: by delayed emotional development; by the need to exert power and control; as the 're-enactment of childhood trauma to undo the hurt'; and as 'narcissistic identification with self as a young child'. On a social/cultural level, emotional congruence is explained by the: 'masculine requirement to be dominant and powerful in sexual relationships'.

Sexual arousal - a 'physiological response' to children's presence or to sexual fantasies involving children (p.39) - is explained on an individual level by a: 'childhood sexual experience that was traumatic or strongly conditioning; and on a social/cultural level by child pornography, and the 'male tendency to sexualise all emotional needs'.

On an individual level, blockage is explained by: a 'fear of adult females'; a 'traumatic sexual experience' with an adult; poor social skills; and marital difficulties. It is attributed to 'repressive norms about masturbation and extra-marital sex' on a social/cultural level. Finkelhor suggests that blockage may be sub-divided into developmental and situational 
blockage. In the former, the individual is 'prevented from moving into the adult sexual stage of development' (p.44); in the latter: 'a person with apparent adult sexual interests is blocked from normal sexual outlets because of the loss of a relationship or some other transitory crisis'.

Disinhibition 'is a requirement' for CSA (Finkelhor 1984: 58). In addition to being motivated to abuse, an offender needs to overcome both internal and external inhibitors. On an individual level, internal inhibitors may be overcome by the use of alcohol or the failure of the 'incest inhibition mechanism in family dynamics'. On a social/cultural level several factors prove germane, including:

'social toleration of sexual interest in children; weak criminal sanctions against offenders; ideology of patriarchal prerogatives for fathers; social tolerance for deviance committed while intoxicated; child pornography; and 'male inability to identify with needs of children'.

'Situational' and 'personality' factors are relevant to 'disinhibition-type explanations to account for child molesting' (p.45). In terms of the former, 'stressors' including relationship difficulties; bereavement; and unemployment all appear relevant to the lowering of inhibitions. With regard to the latter, alcoholism, for example, plays a part. Finkelhor (1984: 45) also draws attention to the relevance of disinhibition to incest theories. Here, offenders abuse females in the family either because they are stepdaughters or because they were separated from the child during their early life. Thus, in either case, inhibitions that would be evident in the relationship between natural father and daughter are lacking.

Whereas the first 2 pre-conditions cited above provide some explanation of offenders' behaviour, Finkelhor also draws attention to factors which are external to the offender and the child victim (pre-condition III); which determine whether or not abuse is perpetrated; and if so, against whom. The level of supervision which a child receives from others is the 'most important' external factor (p.58) or inhibitor. Indeed, attention is drawn to the power of supervision to deflect abuse: 
'Supervision does not mean simply being present with the child at all times. It also includes knowing what is going on for a child, knowing when a child is troubled, and being someone to whom the child can readily turn for help'.

Amongst those exerting a 'restraining influence on the actions of a potential abuser' are the child's family; peers; and neighbours, for example. Of interest, is that 'the influence of third parties appears as an important factor in creating a vulnerability to abuse' (p.58). Finkelhor argues that 'when mothers are incapacitated in some way, children are more vulnerable to abuse' (p.58-59). Mothers may be unable to protect because of absence from the family, for example, due to divorce or ill-health. They may be 'psychologically absent' (p.59) due to relationship difficulties with their husband or children. They may also be vulnerable to abuse themselves from a violent male which renders them incapable of protecting others. The sense in which some mothers were 'absent' on a number of levels, proved of relevance to several offenders in this study.

The following prove central to the overcoming of external inhibitors on an individual level. First, a mother who is ill, absent, abused or dominated by a male, and detached from, or not protective towards their child; second, a socially isolated family; third, poor supervision and 'unusual opportunities to be alone with a child' (p.57); and finally, 'unusual sleeping or rooming conditions'. On a social/cultural level, the following feature: inadequate social support for mothers; women's inequality; deterioration of social networks; and the 'ideology of family sanctity’. Also key are: ‘factors predisposing to overcoming child's resistance' (p.57). The following feature on an individual level here: a deprived or emotionally insecure child who has no knowledge of sexual abuse; a 'situation of unusual trust between child and offender'; and 'coercion'. On a social/cultural level, explanations centre on the paucity of sex education for children; and also on children's 'social powerlessness'.

It appears that many factors: 'overcome the ability of children to avoid or resist becoming victims of abuse, and usually one or more of these things are present in every abuse situation'. (p.60). Children who are needy, emotionally insecure or unsupported, for example, will - due to their vulnerability - be at increased risk of abuse. Indeed, these 
amongst other factors including being socially isolated: 'all erode a child's ability to resist'. (p.61). Additional factors such as the child's age, level of understanding and relationship with the abuser also feature. Requests to comply with a sexual activity, for example, are more likely to be agreed to if the victim is in a close relationship with, and trusts the offender. Of course, in some cases - where force is used - the child's response would have no impact on outcome.

It is clear that Finkelhor's model has many strengths, not least, its emphasis on 'situational and contextual factors' (Ward and Hudson 1998: 50); and its ability to link 'motives to the different phases of offending' (Ward et al. 2006: 27). If there is resistance to considering the factors which preceded and maintained offending, the result is a lack of understanding that it 'emerges from a unique combination of social and psychological factors'; and in a 'patterned way' (ibid.: 30). One of the strengths of Finkelhor's model, is its ability - on a clinical level - to furnish abusers with a 'framework' which enables reflection on offending in a more ordered way. By enabling offenders to develop an understanding of the individual nature of their behaviour, it fosters some recognition that they are individuals who have been convicted of CSA, as opposed to 'sex offenders' who are not amenable to change. In that sense, the model emits a sense of optimism and hopefulness for the future (ibid.).

However, the model is also beset by a number of weaknesses, namely: its failure to explain first, why nonsexual emotional needs (emotional congruence) are met through sexual offending; and second, to provide a detailed account of the origins of that offending. There are two further points to note: first, the overcoming of internal inhibitors is relevant only to a small group of offenders: many are not conflicted by their desire to engage in sexual activity with a child (Ward and Hudson 2000). Second, it fails to:

'provide a comprehensive description of the processes associated with each factor or an understanding of how they interact' (Ward and Hudson 1998: 50). 
In particular, the model is weakened by its general approach and its lack of detail, for example, in relation to 'disinhibition and overcoming external constraints' (ibid.). Notwithstanding such limitations, its application to the data collected for this study - in conjunction with Ward and Keenan's Core Implicit Theories (ITs), outlined below sheds some light on the factors which played a part in the CSA perpetrated by offenders in this sample; and therefore contributes to some understanding of those.

\section{Ward and Keenan's (1999) Core Implicit Theories}

As noted earlier in this Chapter, the focus in Ward and Siegert's (2002) Pathways model is on: 'dysfunctional psychological mechanisms' or processes (Ward and Beech 2006: 60) which lead to the perpetration of CSA. In contrast, Ward and Keenan's (1999) work on Core ITs is characterized by an emphasis on: 'the content of offenders' beliefs' rather than the processes generating them' (Ward et al. 2006: 67). Given this, it proved of greater relevance - as a framework for analysis - to the data collected for this study. In the section which follows, the 5 Core ITs are summarized for contextual purposes, in preparation for their application to offenders' narratives in the Research Findings Chapters of the thesis.

Cognitive distortions (CDs) have been the subject of much debate in the sexual offending field. O’Ciardha and Ward (2013: 5) define these as:

'specific or general beliefs/attitudes that violate commonly accepted norms of rationality that have been shown to be associated with the onset and maintenance of sexual offending'.

Ward and Keenan (1999: 835) drew on existing research on CDs to formulate five Core ITs relevant to child molesters' thinking. The authors note that: 'individuals construct theories about aspects of their world to understand, explain, and control it' (p.825). They purport that 'sexual offenders' implicit theories about their victims are structured around two core sets of mental constructs, 'beliefs and desires'. Beliefs include opinions, ideas, 
convictions, and knowledge. Desires include wishes; wants, goals, and values. These theories, they argue: 'are used to explain, understand, and predict the behaviour of victims and aid in the planning and execution of sexual offenses'(ibid.: 825).

Sexual offenders' maladaptive ITs stem from: 'early developmental experiences' which impact on the way in which they understand their world (Ward and Keenan 1999: 835). Offenders' childhoods are often characterised by sexual and physical violence, and early and inappropriate exposure to sexual activity: this could result in the belief that sex between adults and children is normal. Distorted thinking is evidenced by perceptions of children as 'sexually provocative' (p.821), and as benefiting from sexual abuse. Such beliefs serve to: 'legitimise sexual involvement with children and function to maintain offending' (p.821). Offenders do not perceive themselves as responsible for harming children; children are viewed as sexual; as wanting sex; and as not being harmed by sexual activity with an adult.

The five Core ITs reflect such beliefs and are:

'children as sexual objects; entitlement, dangerous world, uncontrollability, and nature of harm' (p.827).

These are summarised below; and in each case, examples provided by Ward and Keenan are cited for illustrative purposes. It is important to note that child sexual abusers may: ‘hold many or just one or two' of these ITs (p.833).

\section{Children as Sexual Objects}

The belief that people are: 'sexual beings... motivated by a desire for pleasure’ (p.827) is central to this IT. Children are believed to share these characteristics; and to enjoy and desire sexual activity. They are conceptualised as having knowledge which enables them to make informed decisions about engaging in sexual activity with adults. Sexual desire is 
perceived to be natural and intrinsic to children. Thus, sexual activity with an adult is deemed harmless and beneficial.

Examples of distorted statements (CDs) which might be generated by this theory include: 'the child wanted sex'; 'the child seduced me'; 'the child was not harmed'; 'she didn’t say no or tell, so it must be okay'; 'she said yes to my requests, so it must be okay' (p.828).

\section{Entitlement}

The emphasis here, is on the fact that:

'some people are superior to and more important than others. Because of their superior status such individuals have the right to assert their needs above others and to expect that this will be acknowledged and agreed to by those who are judged to be less important' (p.828).

Ward and Keenan (1999: 828) note that:

'the source of this legitimacy might be based on gender, class, or some other factor. For example, men might be viewed as more powerful and important than children and women and, therefore, have the right to have their sexual needs met when they want and with whom they want'.

Here, the offender's beliefs and desires are of paramount importance; the victim's beliefs and desires are either ignored or seen as subordinate.

Examples: 'children are supposed to do what I want and serve my needs'; 'I'm just providing sex education'; 'if I don’t do it someone else will, so it might as well be me’; 'I'm the boss in this family'; 'people do what I tell them and that includes sex' (p.829). 


\section{Dangerous World}

Here, the emphasis is on: 'the world as a dangerous place' (p.829), where others pursue their own interests, exhibiting behaviour which is rejecting and abusive as they do so. Ward and Keenan (1999: 829) draw attention to the two strands evident within this theory. First, the need to:

'fight back and achieve dominance and control over other people. This involves punishing individuals who appear to inflict harm on the offender and. especially, to ensure that his own position is strengthened. Therefore, if children and/or women are perceived as threats and in need of retribution, they may become victims of sexual abuse'.

Examples: 'I had to teach her a lesson'; 'she had no right to question my authority'; 'it was my way of punishing and controlling her' (p.829).

The second 'strand' relates to the: 'unreliability of adults and the dependability of children' (pp.829-830). Here, emphasis is placed on adults as rejecting and untrustworthy; and children as accepting, trustworthy, reliable, loving and caring. Whereas in the first strand, the focus is on achieving dominance and control, here, offenders' believe they are incapable of dominating adults or retaliating against them. Offenders' emotional needs can be satisfied by children who will neither reject nor exploit them.

Examples: 'children can give adults more acceptance and love than other adults'; 'you can’t trust adults'; 'kids really know how to love you'; 'sex between children and adults is very loving' (p.830).

\section{Uncontrollability}

Here: 'uncontrollable factors are thought to leave the offender with deviant preferences that he is unable to suppress or manage'. Factors of relevance here, are: early experiences, such as CSA; other traumatic experiences, for example, parental loss; stress 
and substance abuse. The offender may perceive himself as: 'a victim of factors beyond his control' (p.830).

Examples: 'I did it because I was sexually abused as a child'; I can't control myself, so I'm not responsible'; 'I was high on drugs and alcohol at the time' (p.831).

\section{Nature of Harm}

Ward and Keenan (1999) draw attention to the 'secondary' nature of this IT which, they suggest, is utilised alongside one of the other 4 theories noted above. Two sets of beliefs permeate this IT: '(a) there are degrees of harm and (b) sexual activity in itself is beneficial and unlikely to harm a person’ (p.831).

The first places emphasis on the notion that: 'harm spans a dimension ranging from little or no distressing consequences... to extreme damage...'. Thus, if an offence could have caused more harm: 'any lesser act is viewed as more acceptable and the offender less culpable'.

The second highlights the beneficial nature of sexual activity. Children are not harmed by sex with an adult; any suffering which does result, is likely to have been caused not by the sexual abuse, but by the physical violence which accompanied it, or others' responses to the child's victimisation.

Examples: 'She is asleep so she will never know what I'm doing'; 'she is too young to remember this or know what I am doing'; 'we are only touching, this isn't really sex'; 'just fondling a child is not as bad as penetrating a child’ (p.832).

The Core IT theory is rooted in the notion that some engage in thinking about themselves, their world and others, in a way that renders them vulnerable to sexually offend (Ward et al. 2006). Commentators have noted the strengths of the IT theory - in particular, its 'rigour' when compared with other theories (Sullivan and Sheehan 2016: 77). O’Ciardha 
and Ward (2013: 7), in their review of theories of CDs - including those of Abel and colleagues (1984; 1989), recognised as the first to employ the term CDs in relation to sexual offending (Ward et al. 2006) - found that ITs emerged as 'the strongest overall'. The authors posit that the strengths of the theory lie in its 'strong fertility and heuristic value', as demonstrated by ongoing efforts to measure ITs, and the development of corresponding theories focusing on cognition in other offenders (see, for example, O’Ciardha and Gannon's (2012) work on firesetters). Ward and Keenan (1999: 834), however, have previously acknowledged the limitations of their work on ITs, in particular, noting that it does not provide: 'a comprehensive account of sexual offending'. Rather, other factors might also prove central to the commission of CSA, for example, attachment issues and poor social skills. However, they further assert that adopting an IT perspective to investigate: 'sexual offenders' beliefs about their victims', others, and the world they inhabit, can provide 'a useful framework within which to approach the complex problems of child sexual abuse' (ibid.: 836). The 'fit' between such a framework and the data collected for this study was evident both at interview, and later, during data analysis. Therefore, that framework was utilised as a means of further understanding the complexity of CSA.

\section{Feminist Explanations}

Feminist explanations of sexual offending against children have much to contribute to understanding of CSA, given their emphasis on: 'factors that socially advantage or disadvantage women and children' (Ward et al. 2006: 168). Indeed, in recent decades, they have: contributed to our understanding of sexual offending, male offenders and female victims (Turton 2008); proved key to efforts to end the 'silence' which surrounds sexual violence against women and children; and informed social work practice (Harrison 2006: 376). Moreover, they have been central features of the anti-rape movement and have contributed to: research and theory on sexual offending; prevention; training; and also to the establishment of rape crisis centres (McPhail 2016). They have also proved useful, for example, in understanding how - with the onset of more sophisticated technologies - cyberspace, and the production of child abuse images, has proved 
influential in sexual offending (Harrison 2006). However, while the focus on gender, power and control in such explanations can undoubtedly aid understanding to some extent, these factors do not 'wholly account for the etiology of sexual assault' (McPhail 2016: 314); and such an approach represents an over-simplification of a complex issue (Walklate 2001). Such limitations are explored here.

As already noted, feminist explanations of sexual offending are of some relevance to the research reported in this thesis, for example, in relation to the CSA perpetrated by some offenders; and the extent to which patriarchal power appeared to characterise that. Such explanations are, however, limited by the extent to which they can be applied to male victims; and female perpetrators (Mendel 1995; Turton 2008). They are, however, as outlined, drawn on to some extent during the course of the thesis.

Sociological explanations of CSA are grounded in feminist analysis. The radical feminist approach has proved dominant since the 1980s (Turton 2010), and takes as its starting point the notion that women's inequality and oppression stems from issues related to gender, as opposed to economics and capitalism, as evident in the Marxist feminist approach (Seymour 1998). For radical feminists, the focus is on social and political factors: in particular, the development of rape myths and a culture which condones and excuses male violence (McPhail 2016); the patriarchal nature of society, in which males assume control and power over females; and a State which seeks to maintain the patriarchal nature of family relationships where gender-based social inequality may flourish (Ward et al. 2006: 168). Central here, are the concepts of 'patriarchy, power and masculinity' (Turton 2010: 281). Sexual abuse is deemed to be the: 'concrete expression of the power men have over women'; and 'gender socialisation' results in the development of behaviours and attitudes in males which enable sexual offending. Here, 'gender is the primary factor' in CSA; and patriarchal family relationships, the cause of that abuse (Ward et al. 2006: 169).

Brownmiller's (1975: 14-15) argument that all men exercise social power through sexualised violence permeates the radical feminist perspective. Of course, all men do not 
abuse, and Ward et al. (2006: 170) draw attention to the overly simplistic notion that all men possess power over all women. Rather, they argue that:

'Power is relational, complex and shifting: it has an unstable existence...Power is something that is experienced as a result of autonomy, circumstances, opportunities, knowledge and relationships; it is not possessed or owned. Power, therefore, should be understood in terms of relationships, and analysed according to who has power over whom, why, and how this changes over circumstances and time' (ibid.: 170-171)

Power cannot be attributed to men 'simply because they are men' (p.171). Rather, power emanates from interaction between individuals; and also between individuals and their environment. Wallis (1995: 4), too, makes an interesting point, noting that while there is a fit between CSA and the abuse of power, 'the majority of perpetrators perceive themselves as powerless' (ibid.). Indeed, they often view themselves as failures, both as males and in their ability to engage in appropriate relationships. Thus, they adopt a victim status; children are utilised as 'substitutes for adult partners' because offenders view children as less powerful than themselves (ibid.).

According to Seymour (1998: 415), the feminist perspective provides the: 'best explanation for the sexual abuse of children'. It allows for the examination of the problem 'within its wider social context'; and provides an explanation for extra-familial abuse, while other approaches have failed to do so. For Seymour (1998: 415): 'Patriarchy grants males the power to dominate females in all aspects of their lives' (p.416). Men's social learning focuses on the fulfilment of their needs by others, usually females. Moreover, male socialisation places emphasis on 'a predatory approach to sexuality'; and the use of sex as a means of asserting power over females (Seymour 1998: 416). Women on the other hand: 'learn from an early age that their position is one of dependency and responsibility to submit to the will of males'. As children, females are particularly vulnerable due to their dependency and poor social status. Thus, there is scant possibility that they will be in a position to protect themselves from males wishing to exploit them. 
Indeed, CSA operates 'as a mechanism of social control that trains young females for their oppressed position as adults'; and is: 'a logical extension of the unequal power relationships between males and females' (ibid. 418). While this standpoint is of some value in understanding some forms of CSA, as already noted, it does not account for either female perpetrators, or male victims (Turton 2010).

Another strand of feminist theory (postmodern feminism) is also lacking in its explanation of CSA. Here - as is the case with radical feminism - the emphasis in the main, is on sexual violence against women (Ward et al. 2006). The approach, does however, have some strengths. There is some rejection here, of the generalisations espoused about men's power; women's passivity and virtuousness, and children's powerlessness; and some recognition that gender and power are neither fixed entities, nor inevitable. Moreover, there is some acceptance of the 'variability' and diversity evident among men (ibid.: 173). Radical feminism's simplistic account of CSA is rejected; power and gender are deemed to be 'relational'; and their complexity is acknowledged. Notwithstanding this, postmodern theorists are: 'unable to account for child sexual abuse clearly and succinctly’ (ibid.: 174).

Cossins' (2000) power/powerlessness theory provides an interesting illustration of sociological feminist theory. Cossins proposes that some men allay their sense of powerlessness and demonstrate power and masculinity - in relation to other men - by perpetrating CSA. While useful for its capacity to locate CSA within a 'social context' (Ward et al. 2006: 176), there are also some weaknesses with the theory, namely in its reference to traits associated with 'normative masculine sexuality' (ibid.), including dominance, predatory behaviour, and self-focus. The sexual offending literature indicates that offenders perpetrate CSA for a number of reasons (Ward and Siegert 2002). Some (Ward et al. 1995) have found evidence of 3 focal points in offenders' sexual activity with children - they may: be focused on their own sexual needs (self-focus); perceive the abuse as being of benefit to the child (victim-focus); or perceive the abuse as reciprocal, and a component of a loving relationship (mutual-focus). Cossins' emphasis appears to be on the first focal point (self-focus), and while some offenders may be seeking power 
through the perpetration of CSA, other needs may also be evident including the need for affection and intimacy. It is unlikely, however, that perpetrating CSA will result in the attribution of power in relation to other men. Rather, in Western society, it is more likely that vilification might result (Ward et al. 2006).

It is clear that feminist theories have contributed much to understanding of gender-based violence and CSA. Harrison (2006: 365), for example, extols their capacity to aid understanding of the increasing problem of IIOC by providing:

'an essential framework for understanding how new information and communication technologies are reinforcing attitudes that degrade and dehumanize women and children’.

Male-perpetrated sexual violence, characterized by power and control, is deemed to be as relevant in cyberspace as it is on an interpersonal, familial level. Indeed, it is argued that such violence is: 'embedded in everyday, rather than deviant, patriarchal and masculine practices’ (ibid.:366). While there are some merits in such explanations, they provide only a partial account, with their emphasis on power, control and male socialization to the exclusion of other offence-related factors (Ward et al. 2006), including psychological, environmental and developmental factors, which are integral to alternative explanations (McPhail 2016). Above all, as Turton (2008: 35) notes:

'It is the very strong emphasis on masculinity and power, rooted in the patriarchal discourse, that limits the scope for explanations of female perpetrators'.

Some women's groups have opposed discussion around female sexual offending as 'antifeminist' (ibid.: 36); and as serving the purpose of deflecting attention from male perpetrators (Russell 1984a). Indeed, with regard to CSA, feminist explanations have: 'prioritised heterosexuality so that male perpetrators have become normalised' (Turton 2008: 36). On the other hand, constructions of motherhood and female sexuality displace CSA perpetrated by women 'outside the continuum of expected female behaviour' (ibid.: 
36). Unless such constructions are challenged, female abusers will continue to be labelled as lacking agency; as male-coerced, and suffering from psychoses (ibid.).

\section{Chapter summary}

In this Chapter, the theoretical underpinnings to the thesis have been highlighted; and their relevance to the data collected have been made clear. The following models/theories, were drawn on during analysis of offenders’ narratives:

- Summit's (1983) Child Sexual Abuse Accommodation Syndrome - drawn on in relation to the impact of CSA on victims and their response

- Spiegel's (2003) Sexual Abuse of Males (SAM) model of dynamics and effects of CSA - drawn on for its specific focus on male victims; and in relation to the impact of CSA; and victims' response to it

- Finkelhor and Browne’s (1985) Traumagenic Dynamics Model - drawn on in relation to the impact of CSA

- Finkelhor's (1984) Four Pre-Conditions Model - drawn on in relation to the concept of becoming an abuser: 'the making of a child molester'

- Ward and Keenan's (1999) Core Implicit Theories - drawn on in relation to the 'thinking' and belief systems which support offending behaviour

In addition, feminist explanations were also drawn on to some extent, during the course of the thesis. Each of the models/theories listed above demonstrated some 'fit' with the data collected from offenders at interview, and were thus selected - above other possible explanatory models - for their capacity to interpret the data in as meaningful a way as possible. Of course, each has their strengths and limitations and these have been discussed. In taking the decision to draw on these models/theories during data analysis, 
the aim was not that one should take precedence, but rather, that each should complement the other, in a building-block approach to ‘understanding' offenders' narratives.

Given the enduring nature of sexual violence, Simons et al. (2008: 550) draw attention to the: 'urgent challenge to identify the etiological factors underlying sexual offending'. It is hoped that in examining the narratives of offenders in this sample, with reference to the theories/models cited above, at least some light will be shed on those. The outcome of data analysis may be found in the Research Findings Chapters which follow; and also in the Discussion and Conclusion Chapter, where differences within and between groups of offenders are explored in some detail. 


\section{Chapter 6: Research Findings - Male-perpetrated CSA: Offenders' experiences and perceptions}

\section{Introduction}

In this Chapter, the emphasis is on the accounts of 32 of the 40 men in this sample who reported at interview that they had been sexually abused in childhood by males. The Chapter is presented in 2 parts. In Part1, offenders' experiences of CSA are explored. In Part 2, their perceptions of that abuse; and its impact on them are considered.

Child sexual abuse 'is one of the most hated crimes in the criminal system’ (Briggs 1995: xiii). That the voices of prisoners convicted of CSA should be heard is unpalatable to many. Yet, if we do not take account of how offenders view their own victimisation we will fail victims and provide ineffective interventions. In an attempt to: 'illustrate much of the complexity' of child sexual abusers' lives (Howitt 1995: 2), the emphasis in this Chapter and each of the other Research Findings Chapters, is on individuality; on the men's individual backgrounds and stories as a means of teasing out that complexity.

In the first instance, and before the men's accounts of their experiences of CSA are presented, the focus is on descriptive data relating to their characteristics; their offending behaviour; the characteristics of their victims; and the abuse they perpetrated. 
The 32 offenders

Table 6.1: Demographic characteristics

\begin{tabular}{|c|c|c|}
\hline$(\mathrm{N}=32)$ & & No. offenders \\
\hline \multirow[t]{5}{*}{ Age: } & $18-30$ & 3 \\
\hline & $31-45$ & 18 \\
\hline & $46-55$ & 3 \\
\hline & $56-65$ & 6 \\
\hline & $65+$ & 2 \\
\hline \multirow[t]{5}{*}{ Marital status: } & single & 12 \\
\hline & married & 8 \\
\hline & divorced & 8 \\
\hline & in process divorce & 2 \\
\hline & living with partner & 2 \\
\hline \multirow[t]{8}{*}{ Children: } & 0 & 12 \\
\hline & 1 & 2 \\
\hline & 2 & 5 \\
\hline & 3 & 3 \\
\hline & 4 & 4 \\
\hline & 5 & 1 \\
\hline & 6 & 4 \\
\hline & 9 & 1 \\
\hline \multirow[t]{5}{*}{ Qualifications: } & none & 16 \\
\hline & GCSEs & 3 \\
\hline & ALevels & 2 \\
\hline & Degree & 2 \\
\hline & Other & 9 \\
\hline
\end{tabular}

Robertiello and Terry (2007: 513) note that: 'Like all sexual offenders, child molesters constitute a heterogeneous population of individuals'. Offenders in this sub-sample ranged in age from 23 to 80 years. Most (18) were in the age range 31 to 45 years, with 2 men aged over 65. Just over a third were single (12); 8 were married; 8 were divorced; and 2 men were in the process of divorcing following their conviction and imprisonment. Many (20) of the men had children of their own. 
Evidence suggests that male victims of CSA are more likely to experience educational difficulties than children of either gender who have not been abused (Boney-McCoy and Finkelhor 1995). It is unsurprising therefore, that half (16) of the men in this sub-sample had no qualifications. Five men had either GCSES or A Levels which they obtained at school. Two were qualified to degree level and the remaining men had work-based qualifications. Males who have experienced CSA are also more likely to earn less when in employment; find it difficult to maintain employment; and are often unemployed (Gill and Tutty 1999). Many (19) of the men in this study who were employed prior to imprisonment had been engaged in unskilled, manual work.

Details of the men's offending behaviour follow.

\section{Offending behaviour}

Table 6.2: Offending behaviour

\begin{tabular}{|c|c|c|}
\hline$(\mathrm{N}=32)$ & & No. offenders \\
\hline \multirow{3}{*}{ Previous offending: } & none & 8 \\
\hline & sexual offences (children) & 10 \\
\hline & burglary/theft/fraud & 14 \\
\hline \multirow[t]{3}{*}{ Current sentence: } & $<18$ months & 1 \\
\hline & 18 months to 4 years & 10 \\
\hline & $>4$ years & 21 \\
\hline
\end{tabular}

Eight of the 32 men had no previous convictions. However, 10 had previously sexually offended against children; and 6 of those had served a prison term for those offences. Seven of those 10 men may be defined as 'specialist' (Harris et al. 2009: 1051) offenders, with convictions exclusively for sexual offending against children. The 3 remaining men had also committed a range of other non-sexual offences. In all, 15 men had previously served a prison sentence. Twelve of those had served between 1 and 3 prison sentences; one man had been imprisoned 13 times (not for sexual offences, however). 
Kendall-Tackett and Simon (1987: 243) note: 'the idea that molestation usually involves intercourse' has been sustained by media emphasis on especially violent or unusual cases. This emphasis is in place today (Weatherred 2015). Yet, as Finkelhor (1984: 182) highlights, most child sexual abusers gain gratification: 'not from intercourse but from either manipulating the child's genitals or having the child manipulate their own'. While 9 of the men in this sub-sample had been convicted of indecent assault against their victims - usually involving masturbation - others perpetrated more severe forms of abuse. These included rape; usi; buggery; and incest. Of course, this emphasis on penetrative sexual activity may be due to the fact that, as Finkelhor (1984: 35) points out: 'convicted sex offenders are those who are the most compulsive, repetitive, blatant, and extreme in their offending'.

Many of the men (21) were serving sentences over 4 years in length. One man was serving a 14 year sentence; and 2 others, 10 year terms. Two men had been convicted of offences involving indecent images which were accessed through the Internet; one of those was convicted of possessing and distributing those images and was the only man in this sub-sample to have engaged in non-contact abuse. The other man had also engaged in contact abuse and had been charged with indecent assault. A third man had been convicted of indecent assault and also of filming his victim and making indecent images.

\section{Victims}

In the section which follows, attention turns to the men's victims. Information relating to the number, age and gender of victims is included in Table 6.3, along with details as to the duration of abuse. As noted previously, this information has not routinely been collected in research on CSA. Given this gap in knowledge, as detailed a picture as possible of the men's victims is provided here. 


\begin{tabular}{|c|c|c|}
\hline$(\mathrm{N}=32)$ & & No. offenders \\
\hline \multirow[t]{7}{*}{ No. victims: } & 1 & 17 \\
\hline & 2 & 5 \\
\hline & 3 & 6 \\
\hline & 4 & 1 \\
\hline & 5 & 1 \\
\hline & 6 & 1 \\
\hline & *Info missing & 1 \\
\hline \multirow[t]{6}{*}{ Age of victims: } & 1-5 years & 1 \\
\hline & $6-10$ years & 7 \\
\hline & 11-15 years & 16 \\
\hline & 0-11 months $+1-5$ years & 1 \\
\hline & $1-5$ years $+6-10$ years & 1 \\
\hline & $6-10$ years $+11-15$ years & 6 \\
\hline \multirow[t]{3}{*}{ Gender of victims: } & female & 14 \\
\hline & male & 14 \\
\hline & $\mathrm{m}+\mathrm{f}$ & 4 \\
\hline \multirow[t]{7}{*}{ Duration of abuse: } & 0-11 months & 13 \\
\hline & $1-5$ years & 11 \\
\hline & $6-10$ years & 4 \\
\hline & 11-15 years & 1 \\
\hline & 16-20 years & 1 \\
\hline & 21-24 years & 1 \\
\hline & *Info missing & 1 \\
\hline \multicolumn{3}{|c|}{$\begin{array}{l}\text { *Information missing: in some cases, offenders chose not to provide some details about } \\
\text { their offending }\end{array}$} \\
\hline
\end{tabular}

The majority of men (28) abused between 1 and 3 victims, with just over half (17) offending against one victim. Three men, however, abused between 4 and 6 victims. Half (16) of the offenders in this sub-sample demonstrated a preference for victims in the age range 11-15 years; and 8 abused victims under the age of 10. Six men, however, crossed age boundaries, abusing both pre- and post -pubescent children aged 6 to 10 years and 11-15 years. It is interesting to note, that although evidence suggests that females are more likely to experience CSA than males, 14 men abused female victims and 14, male 
victims. Some men crossed gender boundaries, with 4 abusing both male and female victims.

In 13 cases, the abuse perpetrated by offenders lasted for up to one year. However, 11 other men abused over a period spanning 1 to 5 years; and a further 4 abused victims over a 6 to 10 year period. There were also a number of other men (3) whose abuse spanned many years. One of those men abused a male victim who had a disability, from childhood to adulthood. In the other cases, the abuse perpetrated was tri-generational in character. Here, offenders abused their children first, then their grandchildren.

In the majority (25) of cases, victims were abused in the offenders' - and often their own - home. In the remaining cases, victims were abused in offenders' cars; or in the homes of friends and relatives.

In the section which follows, attention shifts to offenders' relationship to their victims.

Table 6.4: Offenders' relationship to victim/s

$\begin{array}{lll}(\mathrm{N}=32) & & \text { No. offenders } \\ \text { Relationship to victim: } & \text { Intra-familial } & 12 \\ & \text { Extra-familial } & 12 \\ & \text { Unknown } & 3 \\ & \text { Mixed } & 5\end{array}$

Twelve of the men in this sub-sample offended on an intra-familial, and 12 on an extrafamilial level. A further 5 men abused victims across those relationship boundaries: for example, as fathers, grandfathers, uncles and family friends. As might be expected, only 3 of the 32 men abused children who were unknown to them.

Attention now shifts to the CSA offenders reported experiencing: namely, their accounts of that abuse; their perceptions of it; and its impact on them. In order to preserve anonymity, a limited amount of information is provided on each of the 32 offenders; and each man has been allocated a pseudonym. Their accounts are presented verbatim. 
However, details which may serve to identify either victims or perpetrators have been changed or omitted.

The accounts are ordered according to the 3 themes below, which emerged from analysis of the interview data:

- Offenders' experiences of male-perpetrated child sexual abuse

- Offenders’ perceptions of male-perpetrated child sexual abuse

- Becoming an abuser

Data relating to the men's experiences of CSA; their perceptions of that abuse; and its impact on them, are presented in this Chapter. In each case, their account of their experience of CSA is preceded by some contextual information on their age; marital status; and offending. This approach has been adopted in order to highlight consistencies and differences between the men on each of those variables. Moreover, a number of distinctions are made here within and between groups: for example, in relation to those men reporting intra-familial and extra-familial CSA; between those who were abused by other children and young people and those abused by adults; and those abused by males in a position of trust. This is not an approach that has predominated in the literature of sexual offending. Indeed, much of the research on CSA does not distinguish between forms of abuse experienced, for example, intra- and extra-familial abuse; rather, these are often combined within studies (Black et al. 2001). In direct contrast, the approach adopted in this thesis is one of teasing out difference where that may exist; and is a defining characteristic of this research study.

\section{Part 1: Offenders' experiences of male-perpetrated CSA}

Some have pointed to the possibility that abusers' 'claim of victimisation is a defence strategy’ (Wijkman et al. 2010: 144). As noted in the Methodology, others too, draw attention to the possibility of over-reporting due to social desirability in sexual offenders' accounts of their childhood sexual victimisation - a finding not supported by the work of 
Lee et al. (2002). Many of the men in this sub-sample, however, did not disclose, when first asked, that they had been sexually abused; when they did, many were then reluctant to discuss their childhood sexual victimisation in any detail. The accounts of the men who were prepared to discuss the abuse they experienced are presented in their own words. Of course, these accounts may differ from the experiences of the men who were not prepared to talk in any detail about their sexual victimisation; therein lies one of the limitations of this research.

Finkelhor (1984: 166) suggests that:

- like girls, boys are most likely to be abused by males

- boys are more likely to be abused by a non-family member, and 'in conjunction with other children'

- boys are more likely to live in poverty as part of single-parent households and to also experience physical abuse.

All 32 men in this sub-sample had been abused by males, most on an extra-familial level; and in several cases, 'in conjunction with other children' (ibid.). All apart from 3 had been abused by someone they either knew, or were related to.

Table 6.5 provides information on offenders’ childhood sexual victimisation.

Table 6.5: Offenders' childhood sexual victimisation - relationship to perpetrator

$(\mathrm{N}=32)$

No. offenders

Relationship to perpetrator: Intra-familial 5

Extra-familial 19

Mixed

Unknown

3

*Information missing

1

*One man did not wish to disclose who had abused him 
Only 5 of the 32 men had been abused solely on an intra-familial level. The majority (22) had been abused by those outside their own family or household; in 3 cases, by an unknown perpetrator. Eight of the 32 men were abused by neighbours and family friends; and 10 by those in positions of trust. Just under a third (10) had been abused by other children and young people; in 3 cases, by their brothers. Four men experienced both intra- and extra-familial CSA.

Male victims of CSA are more likely than females to experience physical violence in childhood: sometimes this emanates from their abuser and at other times, from those who do not abuse them sexually (Spiegel 2003). It appears that witnessing or experiencing domestic violence may be a risk factor in the later perpetration of violence (Skuse et al. 1998); and may also be a mediator in the process of moving from victim to sexual abuser (Salter et al. 2003). In addition to sexual victimisation, the childhoods of many of the men in this research were also characterised by physical and domestic violence. Some 20 of the 32 men were also victims of physical abuse in childhood. In some cases, this accompanied the CSA they experienced, particularly for those who were abused in residential care; in other cases it was meted out by those who did not abuse them sexually. While the men were more likely to be sexually abused by extra-familial offenders, it appears that they were most at risk of physical violence from those within their own family. Indeed, some 15 of the 20 men experienced this. The childhoods of 3 men were marked out by domestic violence.

Of course, not all victims of CSA become perpetrators. As Finkelhor (1984: 177) posits: 'those who do probably had victimisation experiences that were different in some way, more severe, more unusual, more disturbing'. In an attempt to explore Finkelhor's proposition, in the section which follows, offenders' accounts of their experience of intrafamilial abuse are considered first, followed by their experience of extra-familial abuse. Variations within and between groups are drawn out; and an attempt is made to identify the extent to which their experiences might have been 'severe', 'unusual' and 'disturbing' (ibid.). 
For some, sexual victimisation involved those closest to them; for others, there were multiple perpetrators, some of whom were in positions of trust. What is clear, is that many of these men's lives were characterised by sexual, and often physical, violence from an early age. Indeed, their narratives reveal childhoods which were deficient on a number of levels.

\section{Experiencing intra-familial CSA}

Five of the 32 men experienced intra-familial abuse solely in childhood: namely, Ken, Donald, Ellis, Luke, and Neil. Two of those men (Ken and Donald) had been abused by their fathers; two (Ellis and Neil) by their uncles; and one (Luke) by a male family member whom the offender did not wish to identify. All those men, except for Ken and Donald, subsequently abused children who were biologically related to them. The latter abused on both an intra-and extra-familial level. Interestingly, Ken abused both male and female victims. The childhoods of 2 of the 5 men (Donald and Ellis) were characterised by physical violence. Moreover, Donald's life was also distinguished by domestic violence.

This section of the thesis opens with Ken’s account of his childhood sexual victimisation:

\section{Abused by father}

Ken was 45 and single at the time of interview. He had been convicted of indecent assault against 3 victims: two girls aged 6 and 7 respectively, and a boy aged 8 . The youngest child was related to Ken and the other 2 victims were the children of friends. The offences were committed over a 4 year period at Ken's home; and also at various other locations when he took the children away on trips.

Ken was sexually abused by his father for several years from the age of 6 . He was aware that his father had also 'targeted' and abused some of his friends: 
'I was abused myself by my own father from an early age. The abuse was regular up until I was about 12 or 13 . [It started] from about 6 . He then used to target some of my friends as well. After he stopped on me, I was aware that it had happened to some of my friends as well'.

While at first, Ken was confused as to why the abuse perpetrated by his father ended, he soon realised that as he became older his attractiveness waned, and he became less 'desirable sexually’ (Fischer and McDonald 1998: 926). Glasser et al. (2001) note the trauma which may result for male victims when abuse ends. Ken's sense of rejection was palpable in his narrative, particularly as he recalled his father's strategic approach to victim selection and the fact that he was discarded when no longer of interest:

'I was going through puberty at the time and my father was only interested in prepubescent children'.

Wallis (1995: 11) points out the relevance of this: that abusers often end sexual 'relationships' with their male victims 'abruptly' when the latter reaches maturity and is 'no longer wanted'. This rejection leaves the victim with:

'A distrust of all adults, an undermined morality, a knowledge that he has been abused (damaged goods syndrome) and, in addition, he has been taught to sexualise emotions'.

Donald was 44 and divorced when interviewed. He had previously been imprisoned for indecent assault and gross indecency against 2 male victims aged 6 or 7. Both were unknown to Donald. His most recent offences were attempted buggery, gross indecency and indecent assault against his 3 sons aged 10,12 and 15. All the offences were committed at the family home and the abuse perpetrated against his eldest son lasted 'a few years'.

Like Ken, Donald had been sexually abused by his father from a young age: his abuse had begun at the age of 5 . His father had also physically abused him and he described his childhood as 'very abusive'. His father had engaged in alcohol abuse and was sexually violent to his mother. Donald did not wish to discuss his abuse in any detail, noting that it was 'too personal' for him. 
A number of factors contribute to the overcoming of external inhibitors and the facilitation of abuse (Finkelhor 1984). Amongst these are: a mother who is ill, absent, abused or dominated by a male, and detached from, or not protective towards their child. Donald provided some insight into his family life, which was also characterised by domestic violence, and the fact that his mother provided no support. The sense of betrayal he felt at his mother's failure to protect him came through clearly as he spoke:

'[She] wasn't there when I needed her. My mother didn't know what was going on - not that I hadn't questioned the fact that she didn't want to know. The marriage was very unstable; it was violent, physically and mentally violent. My father was a heavy drinker so the relationship was poor. And from what I gather now at the time my mother did conceive they were mostly forced upon her. My father raped my mother'.

Finkelhor (1984: 59) notes also that: 'Children who live in isolated settings, or who have few friends and few social contacts are at greater risk to abuse'. This observation appears to apply to Donald, who described how isolated he felt as a child; and how, because home was not a place of safety, he constantly ran away:

'I did not have many friends as a child. We were very isolated. I was constantly running away from home’.

\section{Abused by uncle}

Ellis was 23 and single when interviewed. He had been convicted of indecent assault and making indecent images. His victim was his 3 year old niece. The offences were committed at his parents' home while he was babysitting:

'I took her underwear off to take photos of her twice. I took video camera and still photos of my niece’.

As was the case with Donald, Ellis' childhood was characterised by both sexual and physical violence. He had been sexually and physically abused over a 3 year period by his uncle and recalled:

'I was raped by my uncle from the age of 7 to 10 '. 
Neil was 63 and divorced at the time of interview. He had been convicted of indecent assault against his granddaughter aged 9. The offences were committed over a one year period at Neil's home. Neil had been sexually abused by his uncle in childhood and there is some evidence of invalidation (Spiegel 2003) here, in his attempts to minimise the nature of that abuse:

'My uncle used to come and play around with me. He used to play with my privates - that's all. I was about 7 or 8.'

\section{Abused by male family member: identity not disclosed}

Luke was 57 and divorced when interviewed. He had been convicted of incest against one of his daughters. The offences were committed over a 4 year period, beginning when his daughter was 12 years old. Luke had been sexually abused in childhood by a male member of his family. His loyalty was evident in his determination to protect the identity of his abuser, however, and he did not wish to discuss his abuse in any detail:

'I was sexually abused myself when I was about 8 or 9. I don't wish to name the party because it is a member of my family. It happened during one of the summer holidays. We was away from school and down the allotment shed. He was working on the allotment when it happened'.

Having explored 5 of the men's experiences of intra-familial CSA, attention now shifts to the form of abuse experienced by the majority of men in this sub-sample: extra-familial CSA.

\section{Experiencing extra-familial CSA}

The majority (22) of the men in this sub-sample had experienced extra-familial CSA in childhood; with 3 of those 22 being abused by perpetrators who were unknown to them. Over half (12) of the men whose accounts are presented in this section of the thesis had also experienced physical abuse. Moreover, the childhoods of some (Tom and Oliver) were also characterised by domestic violence. Research evidence indicates that sex offenders who have been sexually abused in childhood will have been part of a 
dysfunctional family, in which little support is provided; and where relationships with fathers are poor (Messman-Moore and Brown 2004). Of note, is that several of the men in this sample referred to the latter.

Two men (Alan and Edward) had been abused by family friends and their accounts follow.

\section{Abused by family friend}

Alan was 36 at the time of interview and single. He had been convicted of USI against a 15 year old girl who was known to him. Alan's father was also a convicted sex offender and his childhood was characterised by violence. Alan was one of 8 men in this subsample who had spent time in institutional/residential care. (The remaining 7 men reported that they had been abused in that environment; their accounts of that abuse are presented later in this section of the thesis).

Alan had been 'put into care at aged 4' and his account is marked out by the anger and hatred he felt towards his violent father; and his contempt for his mother who left the family home when he was very young:

'My dad was very violent. Mum left when I was 2 . He beat us for the slightest thing and we were scared to go home sometimes. I have nothing to do with my mother. My brother still sees her but I don't want to know her. I went into care at 4 because of the first offence my father did - sexually molesting young children (girls). As I grew up I started to hate him.

He did it [sexually offended] all his life. He got caught because he attacked a friend's daughters - aged 5, gross indecency. He's in prison. He got a 10 year sentence. I've got a lot of resentment for him because he always put a front on in front of his friends, putting his arm around me and I knew it wasn't true'.

Alan had been sexually abused in childhood by his father's friend and recalled:

'He used to visit us every weekend. They'd been friends for years'. 
Alan did not wish to provide any further detail of the sexual abuse he experienced as a child. He did, however, reflect on his attempts to avoid being abused:

'I told him to stop and I started to cry. I made sure I was out when he came round'.

Of note, is that Alan was one of the few men in this study who demonstrated some 'resistance' to abuse (Turton 2008: 91), by removing himself from the environment within which it was perpetrated.

Edward was 33 and, like Alan, single at the time of interview. He had been convicted of indecent assault against 2 male victims; and indecent assault and buggery against a third. Two of his victims were aged 12 and the third was 9 years old. The offences had been committed at Edward's home and also at his victims' homes.

Edward had been sexually abused in childhood for a number of years by a family friend:

'[I was abused] over a 2 year period when I was about 10 to 12. This man, he wanted me to go to work with him which I did. [It] started off doing bits and pieces and helping. Instead, he was trying to take me elsewhere to assault and bugger me. It went on over a couple of years'.

\section{Abused by lodger}

William was 67 and married at the time of interview. He had been convicted of buggery against his own son and 2 stepsons. His victims were aged 7, 10 and 14 years when the abuse began. He abused his own son from the age of 7 to 14 years. He remarried, and one year later began to abuse his stepsons: one for 6 years from the age of 10; the other over a one year period when he was 14 .

William had been sexually abused by a male 'lodger' from the age of 8 until he was 'about 11 ', and recalled:

'I was buggered by him when I was about 8'. 


\section{Abused by neighbours}

Two men (Mark and Vince) had been abused by neighbours.

Mark was 49 and single when interviewed. He was one of several men who had previous convictions for sexual offending. He had 9 previous convictions for indecent assault against male victims aged between 8 and 11 years; and had previously served 4 prison sentences. Interestingly, his most recent conviction was for indecent assault against 3 female victims aged 7, 8 and 11 years. They were the daughters of a family he had moved in with. As a child, aged 6 or 7, he had been sexually abused by a neighbour and reflected on the forceful nature of the abuse; and at being unable to prevent it. He also distinguished between his abuser's modus operandi (Leclerc et al. 2005) and his own:

'I got abused - I was buggered. I didn't want to but he forced it on me. All my victims, I wouldn't treat them like that'.

Vince was 62 and married at the time of interview. He had been convicted of buggery against his 2 sons. He too, had been sexually abused in childhood by a neighbour, who had also abused his own son and 7 other children in the neighbourhood. 


\section{Abused by other children and young people}

Table 6.6: Abused by other children and young people

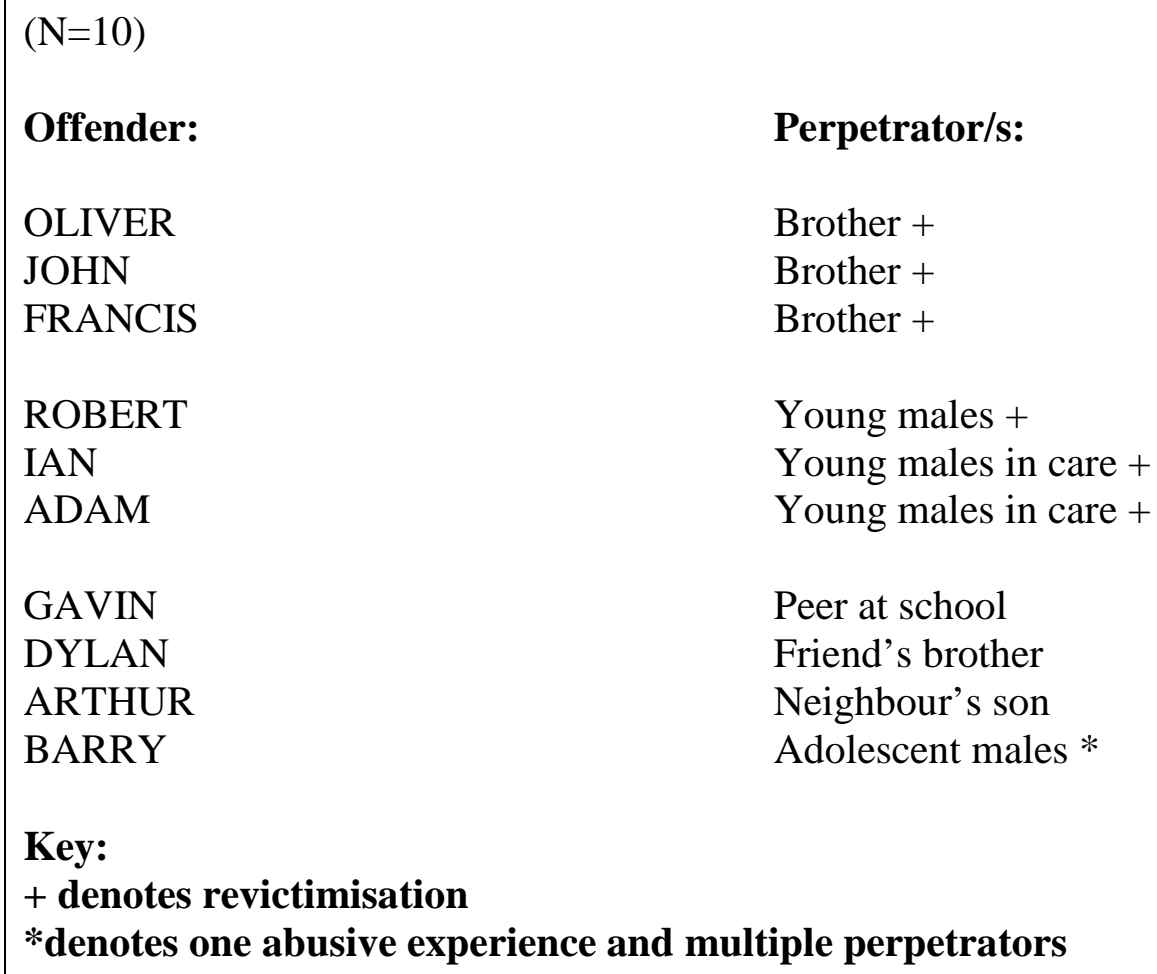

Offender:

OLIVER

JOHN

FRANCIS

ROBERT

IAN

ADAM

GAVIN

DYLAN

ARTHUR

BARRY

\section{Perpetrator/s:}

Brother +

Brother +

Brother +

Young males +

Young males in care +

Young males in care +

Peer at school

Friend's brother

Neighbour's son

Adolescent males *

\section{Key:}

+ denotes revictimisation

*denotes one abusive experience and multiple perpetrators

Although the 'predominant image of sex abusers is of predatory adults' (Hackett et al. 2013: 232), sexually abusive behaviour (SAB) by young people is a significant problem. Indeed, Radford et al. (2011), found that in two thirds (65.9\%) of cases of contact CSA experienced by those aged 0-17 years, the perpetrator was under the age of 18. Finkelhor et al. (2009) highlight some interesting distinctions between adolescent and adult sexual offenders. The former are more likely to offend: against young victims; against males; and as part of a group. They are less likely to rape and more likely to engage in sodomy. Although many - like adult abusers - offend in their own home, they are more likely to offend in a school environment or residential care setting (ibid.; Hackett et al. 2003). In terms of future risk, a minority of those who engage in SAB will subsequently be convicted of sexual offending in adulthood (Hackett 2014; Vizard et. al. 2007). Notwithstanding this, many others have been found to commit violent and non-violent offences as adults (ibid.). The extent to which there is some consistency between 
previous research on SAB and the men's accounts, is explored during the course of this and the following Chapters.

Just under a third (10) of the 32 men in this sub-sample had been abused by other children and young people (in 3 cases by their brothers). Of interest, is that 6 were revictimised in childhood. In addition to being abused by their brothers, Oliver, John and Francis were re-victimised in childhood; all also experienced abuse perpetrated by those in positions of trust. Francis was abused by a youth worker. The 2 other men, Oliver and John, were also abused by care staff. In addition, Oliver was abused by a friend of the family. Robert, Ian and Adam were also re-victimised in childhood. The accounts of those 6 men may be found later in this Chapter, in the section on re-victimisation.

When young people engage in SHB, their victims are usually known to them or related to them (Hackett et al. 2013); and are often their peers or younger children (Boyd 2006). Most perpetrators are aged 12 or over (Finkelhor et al. 2009); and evidence suggests that many abuse children under 11 years of age (Hackett et al. 2013). Four of the 10 men had been abused on an extra-familial level by other children and young people - Dylan, by his friend's brother; Arthur, by a neighbour's son; Gavin, by a male pupil in the same school year; and Barry by adolescent males. While there are distinct differences in age between abusers and their victims in some cases, in others, the abuse perpetrated is peer on peer in nature. None of these men had previous convictions for sexual offences against children.

Arthur was 28 and married when interviewed. He had been convicted of indecency and gross indecency against his niece aged 13. The abuse was perpetrated over a 6 month period at his mother's and sister's homes. Arthur had been sexually abused in childhood:

'by a neighbour's son who was 14 or 15 - I was about 6 or 7 at the time.

Arthur had also been exposed to pornography at a young age by his abuser:

'He showed me a dirty magazine and asked me to show him my willy, and he touched me'. 
Dylan was 35 and single when interviewed. He had been convicted of gross indecency against 1 male victim and indecent assault against 2 other males. Two victims were aged 11 and the other was 12 when the abuse began.

Dylan was sexually abused on a number of occasions by his friend's brother. His only comment on that was:

'He made me masturbate him'.

Gavin was 52, well educated, and divorced when interviewed. He was the only man to have been convicted of non-contact sexual offences: namely, possessing and distributing indecent images of children (IIOC). Research undertaken by CEOP (2012) indicates that these offenders are most often in the age range 19-45 years; white; male; and living with a spouse or partner. Over $50 \%$ have children of their own; and most are either not in work; working in schools or care settings; or in manual or manufacturing jobs. Other evidence (Burke et al. 2002), suggests that these offenders are more likely to be in employment than other child sexual abusers; and to be well-educated (McCarthy 2010). Much (85\%) of the offending is committed at home and the size of collections varies from a handful of images to 2.5million (CEOP 2012). Gavin fits this profile on a number of levels, as illustrated later in the thesis.

Gavin was abused at the age of 12 by a male pupil in the same school year:

'I was forced to expose myself to him and then he was going through all sorts of nasty things: he was manipulating my penis; he got me manipulating his; oral sex; and he wanted to play around with the backside - that was extremely traumatic'.

Barry was 41 and married at the time of interview. He had been convicted of raping his stepdaughter aged 15 . The abuse was perpetrated over a 4 year period, lasting until she reached the age of 19 .

Barry had been the victim of multiple perpetrator rape; he was raped by 3 young males while in youth custody. Barry recalled the violent nature of the abuse; and the physical evidence which resulted. However, the 'pervasive secrecy’ (Summit 1983: 181) and level 
of concealment (Spiegel 2003) inherent in CSA, is illustrated by the fact that Barry did not want to discuss the rape in any further detail, as he had not previously disclosed it:

'I’m not going into detail. They don't know in here [prison]'.

He did, however, provide some detail on his childhood, which had not been happy. He was part of a large family and his childhood was characterised by poverty and physical violence:

'I didn't have a happy childhood. I was beaten by family members: including my mother. I was always blamed for things because I was the oldest'.

Clearly, the impact of the abuse young people perpetrate is significant and should not be under-estimated, given that - as in Barry's case - it can often be severe in nature and may involve force or violence.

\section{Abused by those in a position of trust}

Table 6.7: Abused by those in a position of trust

$(\mathrm{N}=10)$

Offender:

FERGUS

FRANCIS

BOB

STEVEN

CLIVE

HARRY

IAN

CHRIS

JOHN

OLIVER

Key:

+ denotes revictimisation

\section{Perpetrator/s:}

Doctor

Youth worker +

Youth worker +

Professional working in CJS +

Care workers +

Care workers +

Care workers +

Care workers +

Care worker +

Care workers + 
Just under a third (10) of the men were abused by a male in a position of trust. Fergus was one of those 10: he was abused by a medical professional. Two of the other 10 men (Francis and Bob) were abused by youth workers; one (Steven) by a professional engaged in public protection; and the remaining 6 men were abused by staff in institutional/residential care. Interestingly, all except Fergus had experienced revictimisation in childhood. Thus, those 9 men's accounts are presented later in this section of the thesis.

Fergus was 39 and married at the time of interview. He had been convicted of indecent assault against 5 male victims aged between 4 and 8 years; some were related to Fergus and others not. They were his son; a nephew; his godson and his younger brother; and his son's friend. All the offences were committed in Fergus' home, with some abuse against his nephew - lasting over a period of 3 years. His son was aged 6 when the abuse began; his nephew was abused from the age of 7 to 10 years; his godson had been 8 when first abused, and his younger brother 4; his son's school friend was aged 6.

Fergus, like Donald, talked of the sense of isolation he experienced as a child. He reported having been abused by a doctor when aged 7 or 8 . Although he described the abuse as a 'mild incident', it stayed with him and was the basis of his fantasies in later years:

'I was very isolated as a child. As a child I was never socialised...I was never playing with other children. When I went to school I was very isolated, I didn't know how to interact socially. I was abused when I was 7 or 8 years old during a medical examination by a doctor [at school]. It was a very mild incident but it was something that just stuck in my brain. It was something that, in later years, started fantasising about. All the boys from the class were in another classroom dressed in pants and vest and there was a screen halfway across the classroom. Doctor sitting behind it and one by one we went behind the screen and were told to lie down, and he pulled my pants down and fondled me'.

\section{Abused by unknown perpetrators}

Three men (Douglas, Tom and Dan) reported being abused by unknown males. Interestingly, all lived childhoods which were characterised by violence. Dan had 
experienced physical as well as sexual violence from his abusers; Douglas was physically abused by his father; and Tom's family life was marked out by domestic violence.

Douglas was 52 and single when interviewed. He had been convicted of USI against a 12 year old female who was known to him. He had been abused by an unknown male and provided no detail on that.

Tom was 26 and single when interviewed. He had previous convictions - for indecent assault against an 8 year old boy; and child abduction. He had received a prison sentence for the latter. Most recently, he had been convicted of indecent assault and gross indecency against a 10 year old male. Tom was a friend of his victim's family; and the offences were committed at his victim's home. Tom's childhood was characterised by domestic violence:

'I grew up seeing my father beating my mother. I have done the rounds, been to the women's refuges'.

He reported that he had been sexually abused by an unknown male at the age of 8 .

Dan was 33 and single at the time of interview. He had been convicted of indecent assault against his girlfriend's six year old daughter. The offences were committed over a 6 month period at the victim's home. Dan's childhood was characterised by sexual activity from a young age. He talked of being 'sexually active' at the age of 6 with a female friend of the same age: activities which were directed by his friend's older sister; and also of being raped and physically abused by a group of unknown males. His perception of the former contrasted sharply with that of the latter and this is considered in further detail later in this Chapter. Dan did not define the influence of his friend's sister as abusive; and talked positively about the sexual activity which she appears to have directed. He was not, however, prepared to talk in any detail about the male-perpetrated CSA he experienced. There appear to be some parallels here with Barry's experience of multiple perpetrator rape, and his reluctance to discuss that:

'I'll give you a brief insight into the rape. I don't want to go too deeply. I was up on a wall playing with my friends. Somebody threw a stone which went over the 
wall, hit the windscreen [of an expensive car] which happened to be the motor belonging to $X$. Smashed his screen. His men saw me up on the wall, grabbed me and I got taken away. And basically that's it. That's as far as I'm going to say'.

\section{Abuser's identity not disclosed}

Carl was 36 when interviewed and separated from his wife. He had been convicted of indecent assault against his daughter. The abuse was perpetrated over a 6 year period, from the age of 7 to 13 years; and was accompanied by physical violence. Of note, is that there was evidence in Carl's account of 'compensation' or the demonstration of 'overtly male traits' such as aggression (Spiegel 2003); both in his abuse of his victim and in his interaction with other family members.

Carl did not want to discuss his childhood sexual victimisation. He did, however, talk about other aspects of his childhood. He explained that his father was: 'very, very gentle and soft'. However, his childhood was characterised by the physical violence meted out by his mother, and the fear of that:

'My mum was quite an aggressive person. She used to hit out. She used to hit quite hard with different things. I can remember the fear [of that]. The fear of my mother standing at the door shouting at me and knowing what is coming. I got to my teenage years and I had got quite aggressive. When I was aggressive to my children, I used to see my mum in myself'.

Carl referred to his mother as the 'village bike' and believed her behaviour had influenced his view of women:

'There was all sorts of stuff, like, my mum had abortions because she had been made pregnant by other men. That kind of stuff does mess you up and you end up having a very negative attitude towards women, I suppose’.

\section{Experiencing re-victimisation}

Much research on re-victimisation has focused on female CSA victims (Widom et al. 2008). Where research has been undertaken on male victims, re-victimisation rates (relating to childhood re-victimisation) range from 25\%-65\% (Spiegel 2003; 1995). In this study, 13 of the 32 men who had been abused solely by males experienced re- 
victimisation in childhood. Some men were abused within their own family first and then, also on an extra-familial level; others had been abused by more than one abuser who was known to them; yet others had been abused by those in a position of trust, in particular, residential care staff. Indeed, more than half (7) of the 13 men were re-victimised in the latter environment. Table 6.8 provides information on the men's abusers. Their accounts of that abuse follow; and an attempt is made, where possible, to determine from their narratives which factors proved salient in their re-victimisation, as a means of contributing to knowledge on this topic.

Table 6.8: Experiencing re-victimisation in childhood

$(\mathrm{N}=13)$

Offender:

FRANCIS

PAUL

ROBERT

BRIAN

BOB

STEVEN

CLIVE

HARRY

IAN

CHRIS

JOHN

OLIVER

ADAM

\section{Perpetrators:}

Brother + youth worker

Father + neighbour

Neighbours $x 2$

Neighbour + hotel staff

Neighbour + youth worker

Multiple perpetrators, including

male in position of trust

Care workers

Care workers

Care workers + male in care

Care workers

Care worker + 'ex pupil' + brother

Family friend + care workers + brother

Other males in residential care

\section{Re-victimisation: Intra-familial and extra-familial abuse}

Two men (Francis and Paul) had experienced intra-familial abuse, followed by extrafamilial abuse. Francis had first been abused by his brother and then by a youth worker. Paul was first abused by his father - who was also physically violent - and then by a neighbour. A further 2 men (John and Oliver) had also experienced both intra-and extra- 
familial abuse; the latter while in residential care. Their accounts are included later in this section of the thesis.

Francis was 35 and divorced when interviewed. He had been convicted of indecent assault against his son, aged 11; and his daughter, aged 13. The offences had been committed at the family home over a 5 month period. Francis had been sexually abused by 2 males in childhood; first, by his brother and then by a youth worker. The abuse perpetrated by his brother began when he was 12 and lasted until he was 15 . His brother had been 'around 19' when the abuse began. The abuse he experienced from the youth worker began when he was 15. Francis was not prepared to provide any further detail of his childhood sexual victimisation.

Paul was 35 and living with his partner at the time of interview. He had been convicted of indecent assault and abduction of an unknown female victim aged 9, 'in the woods' near Paul's home. Paul's childhood was highly sexualised, and characterised by both sexual and physical violence. He had been sexually and physically abused by his father since the age of 2. His sisters had also experienced CSA, and the anger and frustration he felt that 'nothing was done about' the abuse was clear:

'Fifteen years of abuse. Physical, sexual and mental. Not just on me, he raped both my sisters by the time they were 10 and nothing was getting done about it'.

He talked of how he and one of his sisters used to engage in sexual activity as children. This is unsurprising given the highly sexualised nature of their home environment. He did not define this activity as abusive, however, referring to it as 'messing about', even though his sister clearly took the lead:

'When I was a kid, me and my sister used to mess about. It was more down to her - she seemed to know what she was doing'.

Paul also reported being abused: 'by an old bloke in the village - he was no different to my old man'. 
Paul was extremely aggressive throughout the interview and provided very little detail of his own abuse, or the offences he had subsequently committed.

\section{Re-victimisation: Extra-familial abuse}

Several men had been sexually abused by multiple perpetrators in childhood. Some of those had been abused on an extra-familial level while in residential care and others not. Robert, Brian, Bob and Steven fell into the latter category. Neighbours featured as perpetrators in Robert, Brian and Bob’s case. Robert and Steven's childhoods were also characterised by physical violence.

Robert was 61 and married when interviewed. He had been convicted of USI against 2 of his daughters, and indecent assault against a third; and buggery against his son. The abuse had been sequential, with Robert moving on from one child to abuse another. His daughters were each aged 13 when he began to abuse them, and his son 10 . The abuse against his daughters lasted for 2 years in each case. He had abused his son over a one year period.

Robert had been physically abused by his father in childhood. He had also been sexually abused by 2 different neighbours; first at the age of 'about 4 or 5', and then again at 8 years old. As in Arthur's case, Robert's abusers were adolescent males aged 16 or 17. Robert recalled that in the first instance, his neighbour had: 'dragged me in bed with him and sexually assaulted me'. He explained that he liked his first abuser:

'I never felt any ill feelings towards him for that, in fact I preferred him to his older brother'.

However, his second abusive experience was more traumatic and he described that neighbour as being 'more forceful'. He provided little additional detail about his sexual victimisation in childhood. 
Brian was 57 and divorced when interviewed. He had been convicted of indecent assault against his stepdaughter. The abuse began when she was 13 and continued for the next 4 years. Like Robert, he had been sexually abused by 2 different males during childhood. First, at the age of 9 by a neighbour; and then when he was 15, by a member of staff at the hotel where he took up an apprenticeship:

'My mother asked the neighbour if he wouldn't mind looking after me for the afternoon. And he decided that we'd go for a walk with his dog and he took me down the woods and interfered with me and wanted me to do it with him and I couldn't. Once there were a few tears, he stopped. But he masturbated himself in front of me then. Then, when I was 15 I was sent to be an apprentice in a hotel. The first night I was there, I had to sleep in the same room as the [X] and during the night he came in bed and interfered with me'.

Bob was 45 at the time of interview and married. He had been convicted of indecent assault against his son, and his son's friend, both of whom were aged 7. The abuse had been perpetrated over a 6 month period. (Some years earlier, Bob had been convicted of indecent assault against a 12 year old boy: 'the son of a family who lived in the same building'). As a child, Bob had also been sexually abused by 2 different males: first by a neighbour and then by a youth leader.

Glasser et al. (2001) note that some victims of extra-familial abuse may have experienced physical abuse, rejection, or deprivation within their family, and thus may look outside the family unit to have their needs met. Given this, victims are more easily accessible to abusers, with whom they develop intimate relationships. Bob had a poor relationship with his father, and his narrative provides some indication of the sense in which he valued the relationship with his abuser:

'I had a terrible relationship with my father as a child. I just couldn't get the attention I was after. Looking back it was an obsessive need. I had a difficult relationship with mum. She was also struggling for attention from an absent husband. As we got older, we were aware of the fact that she had a series of affairs with other men.

He [the neighbour] abused me for 4 years, from when I was 7 to 11 . He was a friend of the family. He became a close member of the family very quickly. My brothers and sisters also believe he had an affair with my mother at some point. 
Also, he tried to abuse my brother and sister but he didn't succeed. I sabotaged my brother's situation. I wasn’t prepared to relinquish the attention I was getting (he was 2 years younger than me). My sister just said no'.

Bob described how the abuse began:

'It started by him taking me to work with him as a travelling salesman during the holidays. Dad was at work and mum was busy so they were grateful of the help. He told me I was special and his favourite and one day he started to fondle me. From the age of 8 he started to bugger me till we moved away, when it ended'.

Later, between the age of 11 and 14, Bob was abused by a youth leader along with 3 other boys; abuse he denied when it was investigated:

'The leader of the [X organisation] also abused me from 11 to 14, when I moved from the area of the original abuse. He encouraged boys to have sex with each other. I was involved with 3 other boys: it was fondling, never full sex, but he participated. It stopped when he was caught for offending against another boy. I denied he touched me when asked by the police'.

Steven was 45 and divorced at the time of interview. He had been convicted of indecent assault against his daughter. The offences had been committed in the family home over a 6 year period, beginning when his victim was 8 years old. Steven had been physically abused in childhood by his father, and recalled that his childhood was:

'not a very happy one. Because I was the oldest I was always held responsible for everything that went wrong. My father always belted me around and called me useless'.

Steven had also been sexually abused from the age of 11 to 16 years. Like Bob, he was abused along with other children; and by multiple perpetrators. One of those was a professional in a position of trust; and many others were respected members of his community. Two women were also present at the abuse, which was often filmed or photographed. His account of that abuse was amongst the most disturbing of all those provided by offenders in this sub-sample. It was characterised by a sense of helplessness (Summit 1983); an understanding of the extent to which power and status facilitates and enables abuse to persist across time; and also some recognition of the powerlessness of others, namely the children and young people who were victimised. Much of the detail in Steven's account has been changed or omitted in order to preserve anonymity. 
He explained that the abuse began when a friend of the family - a professional in a position of trust - caught him doing something 'naughty'. From that point, he became trapped in an abusive cycle, which lasted for 5 years, by threats to tell his physically abusive father of his misdemeanour. Those threats ensured that Steven was compliant and the abuse was concealed (Spiegel 2003):

'It started off with a friend of the family - he was a [professional]. He started taking me to somebody's house [to be abused] and he got other people involved. He had photos - somebody had taken photos when I was there. There was 9 of us [child victims] altogether. It started when I was 11 and I think the oldest one must have been about 14, 15. I know there was one father that was abusing his daughter, so she used to cry whenever he was doing anything to her.

There were about 8 men there. Over the 5 years there must have been quite a lot; and there were 2 women as well. One of the women stopped with the children. I remember once, after the abuse I was looking to have a bath. I was trying to scrub myself clean and she came in with my clothes and she was crying - she started apologising. The other woman used to say that nobody could touch her because of her friends [who were powerful people]'.

\section{Re-victimisation: abuse in care}

Sexual abuse perpetrated by those who work with children is a global problem (Colton et al. 2010). At the time of writing (end of May 2016), yet another Inquiry has begun into alleged abuse at Kincora Boys’ Home in Northern Ireland (BBC News 2016). Outside Inquiry Reports, scant evidence is available on victims' experience of institutional abuse in childhood. Indeed, attention has tended to focus on the abuse which victims experienced prior to placement in care; and the perpetrators of that (Timmerman and Schreuder 2014). Even less is known of child sexual abusers' experience of this form of abuse and its impact on them. Therefore, in the section which follows, an attempt is made to address this gap in knowledge by presenting offenders' accounts of their sexual - and physical - abuse within residential care.

Seven of the 32 men in this sub-sample had experienced CSA - which was often accompanied by physical abuse - while in residential care. Clive, Harry, Ian, John, Oliver and Chris were abused by staff. (Ian and John were also abused by other young people in 
care). Adam was abused by his peers in care. Two others of the 40 men (Frank and David) were also abused by males whilst in institutional/residential care but their cases are distinguished by the fact that they were also abused by females. Thus, their accounts of that abuse may be found in Chapter 9.

Children who have been 'looked after' within the care system often have poor life chances (Stein 2006); are more likely to experience poverty; unemployment; and homelessness; and to have a background of offending behaviour (Stein 2004). It is unsurprising therefore, that the majority of men in this group had a history of offending. Some (Chris, Harry and David) had previous convictions for theft, fraud and GBH. Three of the remaining men (Oliver, John and Clive) had previous convictions for sexual offending against children.

Adam had a long history of sexually offending both against family members and also on an extra-familial level. He was 80 and married when interviewed. He had offended against one of his sons; two grandsons; and 3 of his grandson's friends. He was abused at the age of 14 by an older boy while in residential care. This was followed by further abuse from other older boys also in care. Adam explained how it began:

'He was one of the bully boys. He used to come to my bed and play around and then buggery took place regularly. People were scared of him and he picked on me to be with him and then when he went, somebody else wanted to take over'.

As he grew older, Adam developed a 'resistant response' (Turton 2008: 90) to the abuse demonstrated by the use of physical violence:

'As I was getting older I learnt to protect myself [and it stopped]'.

Clive was 58 at the time of interview and single. Like Adam, he had a lengthy history of sexual offending. His victims were all neighbours; they were male and aged between 5 and 8 years. His most recent conviction was for indecent assault against a male victim who was also a neighbour and whom he cared for on weekends. The abuse began when the victim was 2 weeks old and continued for a period of 5 years. 
Clive's account demonstrates the extent to which multiple placement moves accompanied by repeated episodes of sexual victimisation serve to increase a young person's sense of isolation and detachment from friends, family and society (Stein 2006). Clive had been placed in care at 8 years of age having experienced physical abuse from both his father and mother:

'I had enough of being knocked about at home, like black eyes. My father did that [hit me] with the broom handle, his belt, his boots. The thing is, I was put in care supposedly away from this sort of thing. In fact, it was like throwing fat in the fire because we were treated far worse than I actually were at home'.

While in care he experienced both sexual and physical abuse from staff in several different residential homes; and was helpless (Summit 1983) in the face of authority:

'I am talking about teachers, house-masters, house-sisters - they could literally do what the hell they wanted with you'.

He reported 'often' being sexually abused. He was first abused soon after entering care at 8 years old, by the head of a care home:

'I was stripped, given a good hiding. I was made to give him oral sex'.

Shortly after, Clive was moved to another home run by the same staff. There, the same man continued to abuse him. In Clive's narrative, there is evidence of the extent to which helplessness, entrapment and accommodation (Summit 1983) featured in his abuse:

'I was took to his bedroom. I was stripped. I was made to lie on his bed and he buggered me. And after a time of having this sort of treatment, well, I wouldn't say you enjoyed it, you didn't enjoy it - it was just something that you accepted. You would literally do anything they said to save getting a good hiding or being knocked about - it was the way that the place was run. You daren't say no because you had got no escape; you was too scared to run off. You used to see what happened to other boys that had run off '.

Clearly, Clive believed there was no escape for him. Indeed, he was fearful of the consequences of running away from the abuse; and the example made of boys who did that. Six months later, he was moved to another home where he experienced further abuse from staff over a 2 year period: 
'They got another twist to it [there], putting their hands inside your bed at night and playing with you and stuff. Masturbation and all that sort of stuff. It was mainly just masturbation and fondling'.

Yet another move brought more abuse for Clive. Again, there is evidence here of the power differential between staff and children; the helplessness of the latter in the face of those in authority; and of acceptance of the sexual abuse in order to minimise the risk of physical violence:

'Then I went to the big children's home where certain teachers, if they could get you in the right position sort of thing - get you into the big store cupboards and them sort of places, or in school when there was nobody else there - they tended to do whatever they wanted to do with you. Buggery, masturbation, oral sex. Only there they would - instead of being naked all the while - they would have you in things like swimming costumes, swimming trunks, PT shorts. It was just a case of accepting it to save being knocked about. It was easier to let them get on with it and make it look as if you were enjoying it than being knocked about'.

Harry was 38 at the time of interview. He had been convicted of indecent assault against his partner's 12 year old daughter. He had problems with substance abuse and had only recently been released from prison prior to offending against his partner's daughter. He had been in residential care from the age of 4 to 16 years, where he experienced both sexual and physical abuse from the age of 12 for: 'a couple of years'. He reported that he had been: 'touched and masturbated and stuff like that', by several members of staff but was reluctant to provide any further detail on that. He was, however, keen to point out that he had not been the only victim of sexual abuse; and that he was no different to - and no more vulnerable to abuse than - the other boys in the home:

'It wasn't only me. It was happening to other people as well'.

There appears to be some evidence of invalidation (Spiegel 2003) in Harry's account, both in relation to his substance abuse and the 'numbing' effects of that; and his minimising of the CSA he experienced.

Ian was 33 and married at the time of interview. He had been convicted of indecent assault against 2 male victims aged 13 and 14 years. Ian was a family friend and the offences were committed in the victims' homes. Ian had been in residential care since the 
age of 4 and described himself as: 'destructive and hard to handle'. He had been sexually abused from the age of 5 by care staff and also:

'by a resident in the children's home: he was 16. It went on for 2 years until I moved home and then he followed me. It didn't stop until the home closed down. I went to foster parents then'.

Ian had also been physically abused in childhood by his mother and stepfather. His sister acted as his protector:

'My mother threw me down the stairs and my stepfather used to leather us. My older sister used to protect us’.

Chris was 31 and living with his partner when interviewed. He had been convicted of rape and indecent assault against a 15 year old male victim who was a neighbour's son. $\mathrm{He}$, too, had been in residential care as a child, where he was sexually and physically abused. Like some of the other men in this sub-sample, Chris felt unable to cope with the abuse; and that persistently running away was his only means of escape:

'I went to X [home] and I seen quite a lot of things which was not right and things which I couldn’t cope with, so I run away many, many times’.

Like the other men in this group, he had been sexually abused by care staff - including the deputy head - and reflected on how humiliating he found that:

'It is humiliating. It was when I first came in [to residential care]. He called me over to the wings for chicken and chips. I was 13 and I went over there and that was it'.

\section{Re-victimisation: abuse in care and intra-familial abuse}

John and Oliver shared some experiences, as both had been abused while in residential care and also experienced sibling abuse; both also had previous convictions for sexual offending against children. Oliver, however, had in addition, been abused by a family friend. 
John was 41 and divorced at the time of interview. He was the only man out of the total sample of 40 , to have perpetrated abuse with a co-offender. John had been in care from the age of 4 to 10 years, at which point he was fostered. He had 8 previous convictions for sexual offending against children, and one previous prison sentence, for indecent assault against male victims aged between 7 to 15 years. His most recent conviction was for indecent assault against an 11 year old boy who he met through another sex offender:

'He [the sex offender] was going out with a woman who had 2 kids, and the lad we abused was related to the family'.

Both men had previously spent time in prison together and co-offended against their victim, who was already vulnerable having previously been sexually abused by his uncle. The offences were committed at John's home over a 2 year period.

John had first been sexually abused in childhood by his brother; then by a member of staff at a residential care home; and also an 'ex-pupil' at the home. He had also been physically abused by his mother's partner:

'I was hit with belts. Most of us was knocked about with belts, shoes, chucked about. I have seen him [mother's partner] rape one of my sisters and beat her with a belt'.

He described his home life and throughout his narrative, his sense of abandonment and isolation is palpable:

'I was brought up in a slum basically. Dirty house - like a pigsty. We never seen my mother and father - they was always working and my brothers would look after us, they would take us to school. We never got loved; I never seen love in my family. My mum never gave me love'.

John was abused by his brother from the age of 6; this continued throughout his time in residential care and when he was later fostered:

'My brother was having sex with me - we had anal sex and oral sex and that went on until about 11,12 '. 
Simons et al. (2002) note the relationship between exposure to pornography and CSA. Offenders may use pornography during the perpetration of abuse for several reasons: to groom their victims and normalise the abuse; as a means of sexual arousal for themselves and their victims (Quayle and Taylor 2002); and in order to blackmail victims and prevent disclosure (Itzin 2000). One of the residential care staff also abused John - along with other children - and that abuse often involved exposure to pornography:

'Mr X would come to do bathroom duty. He used to check boys' penises and at night he would come into the bedrooms and get certain boys out of bed to take them to his flat. He took me in there a couple of times and abused me. He used to take a couple of boys in there and abuse us - show us dirty videos. I never seen social services.

John also recalled the abuse perpetrated by an ex-pupil and the 'reward' he received for being abused:

' $\mathrm{X}$ was an ex-pupil and he used to come back and have sex with me and give me a bar of chocolate'.

Implicit in John's narrative is a sense of isolation; and the normality of abuse. Like Clive, John appears to have been resigned to the pervasive sexual nature of his childhood. In John's childhood, abuse was an 'everyday thing', and there was confusion as to what was right or wrong:

'The house used to stink. I was just brought up with abuse as an everyday thing. I used to dread it, coming home [to visit], I knew I were going to be abused. I didn't really want to go home; I had nothing to go home for, but I had nobody to talk to. I didn't know, I thought it was an everyday thing - what people did to me. I couldn't say no because I didn't know what was right or wrong. My life was a sexual life. It don't matter where or when, I was being abused. Don't matter what I did, I was being abused'.

John's family life was highly sexualised and he talked of abusing one of his brothers and his sister. He recalled how, when he was fostered, the abuse perpetrated by his brother continued and how those who could have intervened, 'looked away':

'My social worker thought I was happy there. I wasn't, my brother was abusing me. But they looked away. I was always on edge’. 


\section{Re-victimisation: abuse in care; intra-and extra-familial abuse}

Oliver was 37 and single when interviewed. He had been convicted of indecent assault and buggery against a 14 year old male victim who was unknown to him. (He had 2 previous convictions for indecent assault against 2 unknown male victims aged 12 and 13, for which he had received a prison sentence). Oliver's early childhood had been characterised by domestic violence:

'My mum was always getting beaten up off me dad if he went out working and his meals weren't on the table'.

Oliver had been in residential care from the age of 10 and had been sexually abused in 2 residential care homes. This was an environment where sexual activity between peers was the norm; ingrained in the culture of the institution; and directed by staff:

'When you was in the homes you had to play with each other - you were told to. [By] the staff. That's the way I was brought up'.

One of the factors which prove central to the overcoming of external inhibitors to abuse is: ‘unusual sleeping or rooming conditions’ Finkelhor (1984: 57). Prior to being taken into care, Oliver had been abused over a period of 2 years by a friend of the family who moved into the family home and who slept in the same room as him. (Later, Oliver was abused by his older brother who had also been abused by the family friend):

'My father died when I was 8 years old and a friend of the family moved in. We called him uncle, but he wasn't an uncle, he was just a friend of my mother and father. He moved in and that's when sexual abuse took place. He did it to my 2 brothers but I didn't know it at the time. I thought it were just me. He took me upstairs and slept with me all night and I were being buggered 3 times a day then'.

Being placed into care brought no respite for Oliver:

He [abuser] carried on until I were about 10 years old. Then I got put into care. He kept coming to visit me while I was in care saying that he was my uncle. They believed him at the time so he got a job there part-time, looking after other children. I run away from children's homes from the age of 10 years old to 16 . Then, me older brother abused me when I was 14'. 
As was the case with Chris, for Oliver, the only solution to the persistent abuse he experienced in care, was to run away.

Having presented the men's accounts of their sexual victimisation in childhood, attention now turns to the extent to which they disclosed that abuse, or chose not to tell.

\section{Disclosing abuse}

As already noted, disclosure of abuse can prove more of an issue for some victims - in particular males - with 'nonreporting particularly severe' in this population (Finkelhor 1984: 233). There is a dearth of research on males and their disclosure of CSA; variations in disclosure rates; and the responses they receive (Easton 2013). The data reported in this section of the thesis make some contribution to knowledge on these issues. For offenders in this sub-sample, there were two central issues: first, the majority did not feel able to disclose their abuse in childhood; and second, when they attempted to do so the response they received was inadequate or inappropriate, sometimes taking the form of further punishment for those abused in care. In some cases, fear and threats inhibited disclosure. For others, telling would have meant the loss of certain privileges.

\section{'Deciding' not to tell}

Many CSA victims do not disclose due to feelings of guilt and shame; fear of their abuser; fear that those they disclose to may not believe them; a sense of loyalty to family; or a mistrust of professionals (CCE 2015). As already noted, children are more likely to tell if they feel there is: 'an opportunity to talk, and a purpose for speaking, and a connection has been established to what they are talking about' (Jensen et al. 2005: 1395). However, so many victims are not in a position to take advantage of that opportunity because of the fear instilled in them by their abuser. Neil, for example, did not feel able to disclose the abuse he experienced from his uncle. He recalled that a sense of fear, accompanied by a lack of sexual knowledge prevented him from disclosing: 
'No, [I was] too frightened [to tell]. There was no way. I didn't know nothing about it at that age'.

For Steven, a number of factors played a part in inhibiting telling: he did not believe that his mother would have the capacity to protect him; and his abusers were his father's friends. Telling, he felt, was likely to fuel his father's violent disposition:

'[I] couldn't tell my mother because she was highly strung. If she had told my father he would have beat me because it was his mates'.

He explained that being consistently told by his abusers that he, and the other children being abused, would not be believed; and that the abuse was a punishment for bad behaviour, also proved effective in preventing disclosure:

'One of the men said: nobody is going to believe you if you tell. When they abused us they would tell us they were punishing us for being naughty lads'.

Of interest, is that several of the men (William, Ellis, Edward, Luke and Bob) had told no-one of the abuse they experienced until they were imprisoned. William, for example, explained that he was prepared to tolerate the abuse perpetrated by a lodger because he enjoyed 'going fishing' with him and was reluctant to relinquish that pleasure:

'I used to like going fishing with him and in my mind I thought that if I told anyone there wouldn't be anyone to take me fishing again. I never said a word to anybody. The only time I mentioned that was when I was in prison'.

Some evidence suggests that younger children are less likely to disclose (Paine and Hansen 2002); in some cases this is because they are more likely to be intimidated by the perpetrator (Finkelhor 1984). Ellis had been raped by his uncle from the age of 7 to 10 years and threats ensured that he did not tell. Like William, he did not disclose the abuse until he was imprisoned:

'He [uncle] threatened me not to tell anyone. I never told anyone, only the priest in here.'

While Edward and Luke also disclosed to prison staff, Bob - who had been abused by 2 different males in childhood - had only disclosed the abuse perpetrated by his neighbour to his family since being imprisoned. Males are less likely to perceive the sexual activity 
with their perpetrator as abuse (Spiegel 2003). Bob’s comment appears to support that finding, as prior to disclosing, he had not 'acknowledged' the abuse as an 'issue':

'My family are only recently aware of the abuse - my wife, mother and father since I came into prison. I had never acknowledged it as an issue in my life previously'.

He had not, however, disclosed his second abusive experience. Bob explained that the abuse perpetrated by the youth leader ended when a police investigation into wider abuse resulted in him being: 'caught for offending against another boy'. Children who have been abused often retract allegations or deny that they have been abused when questioned by those in authority (Summit 1983). Bob’s reluctance to disclose is illustrated by his denial of that abuse to police during the course of the investigation:

'I denied he touched me when asked by the police'.

Perhaps the sense in which he felt some responsibility for that abuse - due to the fact that he engaged in sexual activity with other boys and also the youth leader - played some part in that denial; and his continued non-disclosure. To have confirmed that abuse had taken place would have resulted in the opening up of Bob's 'part' in that group activity.

\section{Responses to telling}

Some children do try to tell in childhood, and in some cases they are responded to in a manner which enables their recovery from abuse. When compared with females, however, male victims are more likely to be deemed responsible for their own abuse; to be viewed negatively if they disclose; and to be regarded as: 'gay, deviant, and a potential perpetrator' (Spiegel 2003: 100). Males are less likely to disclose CSA; to be protected by their family if they do disclose; or encounter 'parental concern' about the impact of abuse, aside from anxieties about sexual orientation (ibid.).

Victims of CSA make decisions to tell based on an assessment of whether they feel they will be listened to, believed and supported (Cossar et al. 2013). In this research study, the men who did disclose in childhood were often met with inappropriate and unsupportive 
responses. Moreover, consistent with recent findings on the outcome of disclosure (Smith et al. 2015), telling did not always lead to the abuse ending.

The men who did tell of their CSA, made 'purposeful' (Sgroi 1982) disclosures. Alan told his father of the abuse perpetrated by his friend, but recalled: 'he didn’t believe me'. Brian, like Bob, had been sexually abused by 2 different males in childhood and described how he tried to tell of the abuse; and the response he received. On both occasions, he was effectively silenced and told not to say anything further. He spoke first to his mother about the abuse perpetrated by a neighbour: he was not believed and admonished for telling:

'That evening [immediately after the abuse] I told my mother and I was sent to bed because she said, neighbours don't do things like that and I mustn't say anything like that. In those days, being a widow was hard for my mother, a single lady with children and she didn't want no upsets. I wasn't believed and I went to bed quite upset'.

He also reported the abuse he later experienced:

'I reported it to the hotel management and I was given a room of my own but they asked me not to report it to anyone else. That's another one that got away with it'.

In the first instance, perhaps Brian's mother's status and financial circumstances determined her response to his disclosure and his subsequent silencing. The need to avoid 'upsets' within her own community appears to have been more of a priority than protecting Brian: perhaps this stemmed from her sense of her own vulnerability as a widow without a male protector and provider. With regard to the second abusive experience, it is possible that once more, others' needs took precedence. In this case, perhaps the hotel management valued reputation above all else (Hughes-Jones and Roberts 2015). Whatever the objective here, once more, Brian was silenced.

Oliver had been abused by a family friend (he had also been abused by his brother; and while in residential care). When he was being abused by the man who moved into the family home after his father's death, Oliver believed that his mother was aware of what was happening. Garrett (2010: 681), found that 'more than one' of the 8 male child 
sexual abusers in her sample believed that their mother knew of the sexual and physical abuse perpetrated by their stepfather against them or their siblings. In some cases, this sense of collusion may be present because the abuser provides emotional or economic support which was previously lacking. Feminists' attention in this regard focuses on patriarchal power relations and the emergence of collusion due to societal constraints which ensure that women remain oppressed (Joyce 1997). Where mothers fail to protect, many CSA victims: 'view their mothers with intense anger, contempt, and hostility, sometimes blaming mothers for the abuse more than the offenders' (Joyce 1997: 88).

Oliver explained how at first, his mother denied any knowledge of the abuse and how he hated her for that:

'I started to hate my mother. I thought she knew what was going on with this bloke living in that was abusing us but she denied it. Then she admitted it a couple of years ago. She admitted that she knew but she was too scared, swept it under the carpet'.

Fear of the abuser, together with some concern as to what might be lost, appears to have played a part in Oliver's mother's decision not to support him. There appear to be some parallels here with the response Brian received from his mother. In both cases, a sense of betrayal by the person most often deemed to be a protector - a mother - came through in the men's narratives.

Francis' account sheds further light on the mechanisms which promote silence around abuse. He had been sexually abused by his brother from the age of 12 to 15 years. He described how he disclosed the abuse to his parents; and how attempts were made to contain that disclosure within the family:

'I told my parents about it and they had a word with him and that was it. It was still kept in the family. But nothing happened about it. My sister, everybody [in the family] knew about it. It was like, it's all in the family - keep it quiet.'

Francis’ account illustrates how sometimes family members collude to ensure silence, perhaps with the aim of maintaining reputation and standing within a community; to protect both victim and perpetrator from public gaze and investigation (Finkelhor and 
Williams 1988); out of a sense of shame (Hackett and Masson 2006); or fear of 'rejection and hostility' from others (Hackett 2014: 4). Although Francis felt able to tell, he found his parents’ response lacking: ‘it wasn’t enough'. Sadly, he recalled that the abuse did not stop as a result of their intervention. Francis' response was to run away from home on several occasions.

There is evidence here of the 'damage and risks' associated with an attempt to negotiate 'private solutions' to the discovery of abuse (Finkelhor and Williams 1988: 113). In this case, the abuse continued after disclosure and Francis felt that his parents had failed to protect him. As already noted, sibling abuse is the most prevalent and hidden form of intra-familial abuse, with victims less likely to disclose. Thus, it is less likely to be reported to relevant agencies. It will continue to remain undetected if families attempt to deal with the problem themselves; and of course, the corollary of this is that both victims and perpetrators will be denied appropriate support. Key here, is an understanding of the isolation and 'emotional burden' parents may carry in learning of - and attempting to deal with - their child's sexually abusive behaviour (Hackett and Masson 2006: 193); and of course, the secrecy (Summit 1983) implicit in acts of abuse, as well as responses to them.

Given that evidence of the commission of CSA has the capacity to 'disturb' sensitivities; and impact on relationships; family functioning; and reputations, many 'would prefer not to know' that such abuse has been committed (La Fontaine 1990: 45). Of interest, in the cases of Brian, Oliver and Francis, is the centrality of the concept of collusion and what might be lost as a result of acknowledging abuse. This manifested itself in several ways, all of which effectively silenced each victim. Brian was silenced both by his mother and hotel staff. Oliver's mother finally admitted that she knew of the abuse he had suffered many years after it had ended. Fear of the abuser and the loss of that relationship appeared to feature in her inaction and denial. Francis' family appeared to value family reputation above all else and perhaps colluded in order to maintain that. 


\section{Telling of abuse in residential care}

Briggs (1995: ix) notes that males who have been sexually abused in institutions are more likely to perceive that abuse as 'wrong' and to report it. However, only 1 of the 7 men in this sub-sample who had been abused by males in care told of that abuse: Oliver. (One other man, Frank, who had been abused by both males and females, disclosed abuse in institutional care; his account of that may be found in Chapter 9).

As was the case with some of the men in Colton et al.'s research (2002), John felt unable to 'speak out' because of the fear that he would not be believed. He was clearly angry at the lack of support he received when he was being abused while in residential care:

'My house father used to come out of his flat at night and take boys to his flat. Now, it was our word against his and we never spoke up. We couldn't speak out because nobody believed us and I have got to say social services are a waste of money. They are not helping children, so I got angry at that side of the system'.

As already noted, Oliver received an inappropriate response from his mother in relation to the abuse he experienced from a family friend; this left him feeling angry too. Oliver disclosed the abuse he was experiencing in care, to social services. However, he was punished and re-abused for telling. He made clear at interview how that impacted on his sense of feeling 'safe':

'I told social services when I was 13 years of age and got a good hiding for it in the children's home. I got a good caning and locked in my room. I was pinned down and put into a room and stripped naked. You think to yourself, it must be alright to have sex with blokes. As a child it was my experience. Telling somebody? No, you get a good hiding for it. You don't tell anybody again do you? It were 20 years later [that he next spoke of the abuse - to staff while in prison] - even then I didn't think it was safe'.

\section{Summary: Part 1}

In Part 1 of this Chapter, the focus has been on offenders' experiences of CSA; and the extent to which they disclosed that abuse in childhood. Their accounts demonstrate the heterogeneous nature of sexual offending against children; and the need to understand the individual nature of abusive experiences. 
The majority of men (20) in this sub-sample were, or had been, married; or were living with a partner. Twelve were single. Many of the men (20) had children of their own. Ten had previous convictions for sexual offences against children. The childhoods of many of these men were characterised by physical, as well as sexual, violence. Twenty of the men had also experienced physical violence in childhood; and 3 men, domestic violence. Most (22) were abused by extra-familial abusers in childhood (this number includes 3 cases where the perpetrator was unknown). Five men experienced intra-familial abuse; 4 others, both forms of abuse; and one man did not wish to disclose who had abused him.

Data on the age at which their abuse began was not forthcoming from all the men in this sub-sample. However, it appears from the information provided, that many were very young when first abused. Indeed, 18 of the 32 men were 8 years or under at the onset of abuse. Information was available for all 5 men who had been abused on an intra-familial level; they were aged between 5 and 8 years when their abuse began. One other man who had been re-victimised in childhood was aged 2 when abused by his father (before later being abused by a neighbour). Many of those who had been abused on an extra-familial level were also very young when abuse began. One man, who was also re-victimised, was aged 4; another, 5 years; and 10 others were aged 6 to 8 years at the onset of abuse.

Ten men had been abused by other children and young people; in 3 cases by their siblings Moreover, 10 had been abused by those in a position of trust. Thirteen men had been revictimised in childhood; in 7 cases, while in residential care.

As might be expected, given the research evidence, few of the men disclosed abuse in childhood. Decisions not to tell were based on: fear and threats not to tell; a lack of sexual knowledge; the perception that a mother would not have the capacity to protect; a belief that telling might fuel a father's violent disposition; and that abuse was a punishment for bad behaviour. One man, William, was reluctant to relinquish the privilege of 'going fishing' with his abuser, and thus did not tell. Another, Bob, had not previously acknowledged the abuse as an 'issue'. For those men who did disclose in childhood, responses were severely lacking. Brian disclosed 2 abusive experiences: he 
was not believed by his mother; and when he reported later abuse by hotel staff, he was also silenced. Oliver's mother failed to support him when he disclosed abuse by a family friend; and when he told social services of the abuse he was experiencing in residential care, he was punished by his abusers. Francis told his parents of the abuse perpetrated by his brother, but he, too, was silenced and the abuse persisted.

The majority of men (28) had abused between 1 and 3 victims. Three men, however, abused between 4 and 6 victims. Half (16) of the offenders in this sub-sample demonstrated a preference for victims in the age range 11-15 years; and 8 abused victims under the age of 10. Six men, however, crossed age boundaries, abusing both pre- and post -pubescent children aged 6 to 10 years and 11-15 years. Fourteen men abused female victims and 14, male victims. Some men crossed gender boundaries, with 4 abusing both male and female victims.

A number of factors appear to have predisposed these men to CSA and also to revictimisation in childhood: namely, highly sexualised family environments; the isolation some experienced in childhood and the sense in which abuse met an emotional need which was otherwise not fulfilled; experiencing physical and domestic violence; poor relationships with fathers; 'distant', unsupportive or violent mothers; and placement in residential care - an environment where both physical and sexual violence prevailed. These factors are considered in further detail in Chapter 10 (Discussion), where a proposed model of vulnerability to abuse is presented, based on the men's accounts.

Implicit in their accounts is a sense of helplessness, accommodation, and secrecy (Summit 1983); invalidation and compensation (Spiegel 2003); powerlessness, often evidenced by their running away, and betrayal (Finkelhor and Browne 1985); and the normalisation of abuse. These effects, too, are explored further during the course of the thesis. 
Having heard the men's accounts of the abuse they experienced - and in some cases, their attempts to tell of that - next, the focus is on their perceptions of their childhood sexual victimisation and its impact on them. 


\section{Part 2: Offenders’ perceptions of male-perpetrated CSA and its impact}

\section{Introduction}

Here, attention shifts to offenders' perceptions of their childhood sexual victimisation and its impact on them. Once more, a distinction is made between the forms of offending experienced by the men, beginning with intra-familial abuse, in an attempt to tease out difference; and understand individual experience and the 'meaning' attached to CSA.

The humiliation and disgust which some victims feel at having been abused: 'makes it difficult for them to describe what actually happened' (Kahan 1997: 9). It is unsurprising therefore, that some of the men in this study did not wish to discuss their abuse in any detail, or their perceptions of it. Thus, here, the accounts of 17 out of 32 men are presented. Of course, this is a less than complete data set, with information on just over half of the men in this sub-sample available. In spite of this limitation, however, the dearth of evidence on this topic renders even incomplete accounts of value. There is another point to note here: six of the 32 men in this sub-sample had completed the SOTP; and a further 7 were undertaking it at the time of interview. Ten of the 17 men whose accounts are included in this section of the thesis had some involvement in the SOTP. Five had completed the Programme; and 5 were undertaking it. It is important to note at this stage, the possibility that involvement in the Programme had some effect on their capacity - and willingness - to reflect on their childhood sexual victimisation and their perceptions of that.

\section{Perceptions of intra-familial CSA}

Two of the men who experienced intra-familial abuse, Neil and Luke, did not wish to discuss their experience of CSA, nor its impact on them in any detail. Thus, in this section, the focus is on Ken, Donald and Ellis. Although Ken and Donald, too, refrained from providing some details of the abuse perpetrated by their fathers, there is some indication in their narrative as to their perceptions of the abuse and its impact on them. Of 
interest, here, is that each of the 3 men held negative perceptions of the CSA they experienced.

Table 6.9: Offenders' perceptions of intra-familial abuse

+ details of their subsequent offending

\begin{tabular}{|c|c|c|c|c|c|}
\hline Offender: & Abused by: & $\begin{array}{l}\text { Nature of } \\
\text { abuse: }\end{array}$ & $\begin{array}{l}\text { Viewed } \\
\text { abuse: }\end{array}$ & Offence/s & Victim/s \\
\hline Ken & Father I/F & Unknown & $\mathrm{N}$ & $\begin{array}{l}\text { Indecent } \\
\text { assault }\end{array}$ & $\begin{array}{l}\text { fx2 } \\
6+7 y r s \\
m x 1 \\
8 y r s \\
\text { I/F + E/F }\end{array}$ \\
\hline Donald & Father I/F & Unknown & $\mathrm{N}$ & $\begin{array}{l}\text { Attempted } \\
\text { buggery + } \\
\text { Indecent } \\
\text { assault + } \\
\text { Gross } \\
\text { indecency }\end{array}$ & $\begin{array}{l}\text { mx3 (sons) } \\
10 ; 12 ;+ \\
15 y r s \\
\text { I/F }\end{array}$ \\
\hline Ellis & Uncle I/F & CAP & $\mathrm{N}$ & $\begin{array}{l}\text { Indecent } \\
\text { assault + } \\
\text { Making } \\
\text { indecent } \\
\text { images }\end{array}$ & $\begin{array}{l}\text { fx1 (niece) } \\
\text { 3yrs } \\
\text { I/F }\end{array}$ \\
\hline \multicolumn{6}{|c|}{$\begin{array}{l}\text { Key: } \\
\text { CAP = contact abuse with penetration } \\
\text { CAWP = contact abuse without penetration (Erooga et al. 2012) } \\
\text { Unknown = offender did not wish to discuss nature of abuse } \\
\mathrm{N}=\text { Negative perception of abuse; } \mathrm{P}=\text { Positive perception of abuse } \\
\mathrm{f}=\text { female; } \mathrm{m}=\text { male; } \mathrm{I} / \mathrm{F}=\text { intra-familial CSA; E/F = extra-familial CSA } \\
\text { (In each case, the victims identified here relate to offenders' most current offence and } \\
\text { subsequent imprisonment). }\end{array}$} \\
\hline
\end{tabular}

Briggs (1995: 169) suggests that:

'Because boys accept responsibility for what happened to them and believe their abusers' explanations that they are either educating them about sex or demonstrating their love, male victims seldom have hostile feelings towards the abusers who wrecked their lives'. 
This assertion does not seem to apply to the majority of men in this sub-sample, who described their abuse in negative terms. Three of the 5 men - Ellis, Ken and Donald who had been abused on an intra-familial level reflected on their negative perceptions of that and its impact on them. In turn, they spoke of the physical pain they endured; becoming fearful of adult males; and the 'mental scars' which resulted from their abuse.

Ellis had been raped by his uncle in childhood. The abuse had lasted for several years and like Edward and Bob - who had experienced extra-familial abuse and whose accounts are included later in this section of the thesis - he reflected on the physical pain he had experienced as a result of the abuse:

'The pain I went through. People should be made aware of how much pain a child goes through. There should be more understanding of what children go through when they're abused'.

Spiegel (2003: 73) notes the 'pervasive and enduring' fear that some male victims experience following abuse. Ken recalled that as a result of the abuse perpetrated by his father, he became fearful of adults and of males in particular who, he expected, would want to abuse him:

'I felt as though I couldn't talk to adults generally. I always thought that all men were probably like my father - would probably want to sexually abuse me’.

As already noted, Donald did not wish to discuss the abuse he experienced from his father in any detail, as he felt that it was 'too personal'. This brief comment, however, provides some indication of the strength of his feelings about the abuse and the persistent nature of its impact:

'With physical abuse, the scars heal but the mental scars stay with sexual abuse'. 


\section{Perceptions of extra-familial CSA}

Some of the men who experienced extra-familial CSA did not wish to discuss their experience or perceptions of abuse, nor its impact on them in any detail. Eight others, however, welcomed the opportunity to reflect on their own abuse in the hope it might aid their understanding of why they had offended: this issue is considered in further detail later in the thesis. As indicated in Table 6.10, 5 of those 8 men - like Ellis, Ken and Donald - also held negative perceptions of the CSA they experienced. Perhaps this is unsurprising, given that all 5 had experienced penetrative CSA. 
'I was sexually active at the age of 6, which I thoroughly enjoyed and secondly, I was also raped when I was 6 which I obviously did not enjoy. Sexually active - I used to stay with my friend [who was also 6] and she had an older sister who was 14 and she basically told me what to do to her sister and it became a regular occurrence that I would have sexual intercourse with her and oral sex with her and that went on for about 18 months'.

Dan was clearly finding it difficult to deal with the rape he had experienced; and reflected on the enduring impact of that, describing it as a 'choking weed'. As he spoke, he drew parallels with his victim's attempts to overcome the effects of the abuse he had perpetrated:

'I had treated my rape in my own way and I thought I'd shut it all out. If she's [victim] not strong enough to push it away and try to get rid of it, try and bury it, it's going to come up and grow and grow. The more it grows the more confused she's going to be. It's like a choking weed. I thought I'd got rid of my choking weed. After 27 years I thought I'd got rid of it but it was only the other week when I realised there was another little bit of weed hanging on to my shoulder. It can come up and bite you when you least expect it'.

In contrast, he recalled that he found the sexual activity he engaged in as a child with his sister's friend comforting. While the adolescent female was clearly perpetrating abuse, based on definitions which place emphasis on age and developmental differentials between victim and abuser, Dan did not define that activity as abusive. Therefore, his case and these data are not included in the Research Findings Chapter on femaleperpetrated CSA. For Dan, that sexual activity was harmless and comforting; this is in direct contrast to his recollection of the male-perpetrated abuse he experienced:

'It didn’t do me any harm. I was quite happy as long as I wasn't being hurt. [There was] a feeling of comfort'.

Edward reflected on the persistent and pervasive nature of the effects of his CSA:

'Long term: sexual problems; trusting people. And then there is nightmares - they go on for years. You can feel different, isolated, even if you have got friends you never feel part of any group - you always feel different from them. Difficulty in getting partners; or settling down with anybody. Feeling suicidal as well because you might not be able to deal with what has happened to you. You know, there is always a constant reminder of what has happened - it is not something that you truly forget. Even if you believe you have, you never really do’. 
Cortoni and Marshall (2001) argue that child sexual abusers learn to use sex as a coping mechanism to lessen the pain of the CSA they experienced. There appears to be some evidence of this in Edward's narrative. Edward had completed the SOTP and demonstrated some insight into his offending. Like Ellis, Edward reflected on the physical pain which accompanied abuse. He also recalled his efforts to deal with that, and to survive, by blocking it out and convincing himself that he had not been hurt. The extent to which Edward engaged in a process of distorting reality, and invalidation (Spiegel 2003), comes through in his narrative:

'[I know] how much pain there is involved with abuse. I already knew that when I was a child, what it feels like. But to carry on living I blocked that out and I was telling myself that I wasn't hurt by it. And I think because it never came out, I never had help'.

Edward was aware of the 'damage' that his attempts to deny the pain and hurt associated with his CSA had caused; and how this had impacted on his offending. He also recognised the need to 'sort out' and 'understand' his own CSA and subsequent offending:

'So then I convinced myself that I wasn't hurt by it and it gave me another CD to carry on abusing others. I think if I had had help, I don't think I would have carried on to abuse. If I actually worked with it so I can understand it, it wouldn't have been shut away. But I actually sorted it out by telling myself that it never existed'.

Certain circumstances may give rise to the emergence of 'traumatic memories' of the abuse which can prove intrusive; these may present even where a significant amount of time has elapsed since the abuse ended (Spiegel 2003: 171). For Steven, who was abused by multiple perpetrators over several years, aspects of his abuse - including certain smells and situations where he is part of a crowd - persisted as 'triggers', engendering a sense of sadness and making his abuse extremely difficult to deal with:

'I remember when I was being abused there was always a certain smell about them - they were never over-clean. That's one thing that sticks in my mind is the smell. It's sad, if I was on the outside now and there was a crowd of people, something in the back of your mind clicks [and the abuse comes back]'. 
William valued the attention he received from the neighbour who abused him, in particular, the fact that he took him fishing. However, he also recognised that the abuse had a negative impact on him:

'I had friends but I wouldn't keep them long. I'd quarrel or fight with them. When someone got close to me I'd try to push them away...I was always in trouble pinching things - from about the age of 10 '.

Gavin had been disgusted and repulsed at the sexual activity he was forced to engage in as a child by a male pupil in the same school year:

'My reaction to having a boy approaching me was total revulsion, repugnance'. As evident in Table 6.11, 2 of the 8 men expressed mixed views, and one, positive views of the extra-familial abuse they experienced in childhood; their accounts of that follow. 
Table 6.11: Offenders' perceptions of extra-familial abuse

+ details of their subsequent offending

\begin{tabular}{|c|c|c|c|c|c|}
\hline Offender: & Abused by: & $\begin{array}{l}\text { Nature of } \\
\text { abuse: }\end{array}$ & $\begin{array}{l}\text { Viewed } \\
\text { abuse: }\end{array}$ & Offence/s & Victim/s \\
\hline Fergus & Doctor E/F & CAWP & $\mathrm{M}$ & $\begin{array}{l}\text { Indecent } \\
\text { assault }\end{array}$ & $\begin{array}{l}\mathrm{mx} 5 \\
4-8 y r s \\
\mathrm{I} / \mathrm{F}+\mathrm{E} / \mathrm{F}\end{array}$ \\
\hline Robert & $\begin{array}{l}\text { Neighbours } \\
\mathrm{x} 2 \mathrm{E} / \mathrm{F}\end{array}$ & $\begin{array}{l}\mathrm{x} 1 \mathrm{CAP} \\
\mathrm{x} 1 \mathrm{CAWP}\end{array}$ & $\mathrm{M}$ & $\begin{array}{l}\text { USI + } \\
\text { indecent } \\
\text { assault } \\
\text { (daughters) } \\
\text { Buggery } \\
\text { (son) }\end{array}$ & $\begin{array}{l}\text { fx3 } \\
\text { (daughters) } \\
\text { each at 13yrs } \\
\text { mx1 (son) } \\
\text { 10yrs } \\
\text { I/F }\end{array}$ \\
\hline Bob & $\begin{array}{l}\text { Neighbour + } \\
\text { Youth } \\
\text { worker E/F }\end{array}$ & $\begin{array}{l}\mathrm{x} 1 \text { CAP } \\
\mathrm{x} 1 \text { CAWP }\end{array}$ & $\mathrm{P}$ & $\begin{array}{l}\text { Indecent } \\
\text { assault }\end{array}$ & $\begin{array}{l}\text { mx2 } \\
\text { (son + son’s } \\
\text { friend) } \\
7 \mathrm{yrs} \\
\mathrm{I} / \mathrm{F}+\mathrm{E} / \mathrm{F}\end{array}$ \\
\hline \multicolumn{6}{|c|}{$\begin{array}{l}\text { Key: } \\
\text { CAP = contact abuse with penetration }\end{array}$} \\
\hline \multicolumn{6}{|c|}{$\begin{array}{l}\mathrm{N}=\text { Negative perception of abuse; } \mathrm{P}=\text { Positive perception of abuse; } \mathrm{M}=\text { Mixed view of } \\
\text { abuse }\end{array}$} \\
\hline \multicolumn{6}{|c|}{$\mathrm{f}=$ female $; \mathrm{m}=$ male; $\mathrm{I} / \mathrm{F}=$ intra-familial CSA; $\mathrm{E} / \mathrm{F}=$ extra-familial CSA } \\
\hline \multicolumn{6}{|c|}{$\begin{array}{l}\text { (In each case, the victims identified here relate to offenders' most current offence and } \\
\text { subsequent imprisonment). }\end{array}$} \\
\hline
\end{tabular}

\section{Positive perceptions of CSA}

Glasser et al. (2001) note that children who experience extra-familial abuse often feel isolated from their families. This sense of isolation seems to apply to $\mathbf{B o b}$, who was the only man in this group to view his abuse positively as a means of gaining care and attention. This was in spite of the physical pain which accompanied the abuse. As a child, Bob had a poor relationship with his father and had been desperate for 'attention'. That level of need perhaps rendered him vulnerable to abuse by 2 men: first, a neighbour and then a youth leader. He recalled the abuse perpetrated against him by a neighbour over several years and the extent to which he was 'hurt' by that. That hurt emanated not from 
being beaten but from the physical and intimate nature of the sexual activity perpetrated by an adult on a young child:

'From the age of 8 he [neighbour] started to bugger me till we moved away, when it ended. The sexual abuse I suffered was very physical. He never hit me but he hurt me'.

Bob appears to have tolerated pain and viewed his abuse positively as a means of gaining care and attention. Briggs et al. (1995) note that this factor may be central to our understanding of the intergenerational transmission of abuse. In spite of the physical pain he experienced, Bob had perceived his abuse as 'normal'; it made him feel 'special' and provided him with the care and attention he lacked from his own father:

'I was very much in love with my father and he was a very busy person. I had to share his attention with brothers and sisters and I didn't like that. The man across the road offered a deal - if you let me abuse you, I'll give you love and attention. He made me feel special; he groomed me in that way'.

Although Bob's father lived at home, he was often absent. It is possible therefore that Bob's 'desire for closeness' placed him in a position where he was 'afraid to refuse the sexual advances' of his neighbour because if he did so, there would be no-one to care for him. Of interest, is Bob’s comment earlier in this Chapter, when explaining why he had not disclosed this abuse in childhood, that he had not seen it as an 'issue'.

He recalled how behaviour problems resulted when the abuse ended at the age of 11:

'I was sent to see a child psychologist at 11 . My behaviour had started to become a problem at school - stealing and rudeness. It was a character change. I had a terrible relationship with my father as a child. I just couldn't get the attention I was after. Looking back it was an obsessive need. I had a difficult relationship with mum. She was also struggling for attention from an absent husband'.

It is possible that the behavioural issues which manifested themselves when the abuse perpetrated by his neighbour ended, together with his unmet emotional needs, rendered him vulnerable once more to abuse by the youth worker. 


\section{Mixed perceptions of CSA}

Two other men who had been abused on an extra-familial level - Fergus and Robert had mixed views of the abuse they had experienced. Fergus recalled that it was an exciting but also frightening experience. Robert reflected on the differences evident in the nature of the abuse he experienced from 2 young neighbours.

Fergus reported being abused during a medical examination at school and his sense of confusion comes through in his narrative. He recalled finding it an 'exciting but frightening' experience:

'There was something exciting but frightening at the same time and I wasn't sure about talking about it. I started fantasising about it in my teenage years - it was something I found quite exciting. I think it ties in with my feelings of isolation and rejection as a child by my peers and it grew into feelings of excitement at the thought of non-consenting sex. There were thoughts about the situations where I had the power and control, and that was fed by this memory, this incident that occurred when I was 7 or 8 '.

Robert had been sexually abused by 2 different adolescent neighbours, first at the age of 4 or 5 and then at 8 years old. He explained how differently he perceived those experiences. In the first instance, he 'didn't feel bad'; however, anger characterised his response to the second abusive experience which, as noted earlier, was more traumatic and involved force:

'I was sexually assaulted by the neighbour [first] but I didn't feel bad about it. With the other one [second neighbour], I was just angry at him’.

\section{Perceptions of extra-familial CSA: abuse in care}

Seven men had been abused by males while in care. Six of those expressed their views on that abuse. Table 6.12 includes some detail on those men. 
Six of the men who had been abused while in residential care provided some indication of their perceptions of that. The majority had experienced penetrative CSA and while all clearly held extremely negative views of their abusive experiences, 2 of the men expressed some confusion. Oliver, for example, talked of enjoying the abuse and also 'hating it' sometimes. The remaining man (Adam) also reflected on the fact that he 'got pleasure' from the abuse, in spite of the pain and the disgust he felt. Their accounts may be found towards the end of this section of the thesis.

Clive had been sexually and physically abused by care staff at several residential homes from the age of 8. As was the case with Ken, Clive's abuse resulted in a 'fear' of adults'; and in his view, had scarred him 'for life'. Like Steven, he talked of persistent reminders or 'triggers' which 'bring it all back':

'I have never been able to forget my childhood. I mean 8 years in care is a long time and when things happen like they did when I was in care - 8 years of it - it sort of scars you for life. As a rule, I don't even think about it but suddenly you will see something that will bring it all back - not sexual or anything like that - it's just certain things are embedded in your brain'.

He believed that he had learnt to accept his own abuse as a means of survival (Summit 1983):

'What went on, what was done to me. It got to the stage where you sort of don't look at it as anything wrong. It is something you tolerate and I think that that's where my trouble is. I have got to break that link between that and normal life. At the time, it was the only way I could live. You didn't see the outside world. To you, the outside world was the other side of that fence. You just learned to accept it. You knew that as long as they could do what they wanted to you, you weren't going to be knocked about and you learn to accept it’.

John found it extremely difficult to have a relationship with a female following his abuse, and was confused about his sexuality:

'I used to think I was gay until I got married. I found it hard to make sexual contact with a woman for years because all I had going round in my head was abuse by men and older boys. I had no way of working round it, getting away from it'. 
Harry was reluctant to talk in any detail about the abuse he had experienced while in residential care. In this case, one sees the benefit of engaging in in-depth individual interviews with offenders, which allow them the time to decide whether - and when - to talk about their abuse. Towards the end of the interview, Harry began to talk, without prompting, about historical abuse in general; and the extent to which cases from the 1970s were now coming to light. Perhaps introducing that topic into the 'conversation' allowed him to make the connection with his own abuse. That link made, he went on to describe how the abuse he had experienced had affected him; and left him feeling 'ashamed' and lacking in confidence. Like John, he found relationships with females difficult:

'It affected me quite badly, made me ashamed of myself, and no confidence in myself. I suppose it carries through to what I have done and why I never had no confidence to have relationships with girls when I was younger.'

Ian had been sexually abused by an older boy, who was aged 16, and also by staff in residential care. While he provided little detail about his own abuse - or indeed, other issues raised during the course of his interview - his narrative was permeated by the harm and shame which resulted from his sexual victimisation as a child. There are parallels here with the accounts of men in Colton et al.'s (2002: 544) research - who had been abused in residential care but were not sexual offenders - who also referred to feelings of shame and 'being dirty'. Ian explained how 'screwed up' he felt; and how feelings of shame prevented him from seeking help:

'I put them [victims] through what I went through and it shouldn't have happened. My life was so screwed up and I screwed their lives up. I should have asked for help but I was so ashamed'.

For Ian, 'counselling' or 'talking to somebody' is absolutely crucial. The inevitability of the harmful impact of abuse comes through in his account:

'The more you lock it up, the more it chews you up. They're [victims] not going to put this behind them. It doesn't get better. It makes you feel angry and dirty'.

Oliver had been in residential care from the age of 10 and had been sexually abused in 2 residential care homes. He described how he constantly ran away to escape the abuse; 
and how confused he felt about being abused. Like Bob, he reflected on the sense in which he was prepared to tolerate the physical pain he experienced in return for some much-needed attention and 'treats'. Oliver also recognised the extent to which, after the first year of abuse, he normalised it:

'When it was happening to me, I was enjoying it and I hated it sometimes. I hated it for the first 12 months but when I were about 9, 10, 11 year old I just put it down as normal and it must be right; no-one is complaining. It's not hurting me anymore and I'm enjoying it. They force it on you and no-one believes you when you tell them, and you get a good hiding, so you think it's normal; you believe it even if you know it's wrong. You are confused and mixed up - it must be OK to do it because you are getting presents and you are treated for a couple of hours pain a night '.

A sense of powerlessness and entrapment permeates Oliver's narrative. Moreover, there is also evidence of his 'accommodation' to the abuse (Summit 1983). According to Oliver, the abuse he experienced has left him feeling:

'Embarrassed. You are ashamed; you feel dirty'.

The remaining man, Adam, was abused at the age of 14 by an older boy while in residential care. This was followed by further abuse from other older boys also in care. He recalled how pervasive abuse was in that environment; and that in time, he too, 'got pleasure' from it in spite of the 'pain and disgust' he felt:

'I know the way I was treated when I was a boy of 14 - although there was pain and disgust at what was happening, I did come to like it. I got pleasure out of it. When I was abused I was in rooms with about 8 to either side - a dormitory. And this thing [abuse] went on all over the place'.

\section{Summary: Part 2}

In Part 2 of this Chapter, the focus has been on offenders' perceptions of the CSA they experienced and its impact on them. The men's narratives shed light on the diverse nature of impact in a particular population. Briggs and Hawkins (1996) posit that adult male victims who hold positive perceptions of the CSA they experienced are more likely than those who hold negative perceptions to be convicted of sexual offending. This does not 
appear to hold true of the men in this sub-sample: the majority of whom reported negative perceptions of their childhood sexual victimisation.

Just over half (17) of the 32 men were prepared to discuss their perceptions of CSA and its impact on them. The majority (14) of those held negative perceptions of their abuse. This number includes 3 of the 5 men who had been abused on an intra-familial level; and 11 of those who had been abused on an extra-familial level (the latter includes 6 men who had been abused in residential care). Only one man, Bob, viewed his abuse positively. Fergus had mixed views; and Robert, who had been abused by 2 different males, viewed one experience negatively, and had mixed views of the other.

With regard to impact, the 3 men who had been abused on an intra-familial level referred to physical pain; a fear of adult males; and 'mental scars' resulting from the abuse. The 8 men who had been abused on an extra-familial level also reflected on the persistent and pervasive effects of CSA: including, confusion; physical pain; sexual problems; an inability to trust; nightmares; isolation; feeling suicidal; revulsion; and difficulties with relationships with peers. The 6 remaining men, who had been abused while in residential care, spoke of: the physical pain associated with abuse; a fear of adults; the abuse having a life-long 'scarring' influence; difficulties with relationships with females; confusion about their sexuality; feeling ashamed; lacking in confidence; embarrassment; disgust; and feeling angry and dirty.

In Chapter 7, attention shifts to the men's accounts of 'becoming an abuser'. 


\section{Chapter 7: Research Findings - Becoming an Abuser}

\section{Introduction}

In the previous Chapter, offenders' accounts of their childhood sexual victimisation by males; their perceptions of that; and its impact on them were examined. Here, their accounts of becoming an abuser are explored. First, an attempt is made to determine to what extent the men identified links between their own childhood sexual victimisation and their offending. Then, attention shifts to exploring the presence of Core ITs (Ward and Keenan 1999) in their narratives as a means of understanding the belief systems which supported the perpetration of abuse.

As noted in the Methodology, during interview, all the men were asked the following questions in order to determine their understanding of the origins of their sexual offending. The questions were designed to incorporate any abusive or negative experiences in childhood, both within and outside the family; and also to enable a focus on abusive or negative experiences with children and young people:

- To what extent do you see a link between your own family experiences as a child and your offences against children?

- To what extent do you see a link between your relationship with childhood friends, inside and outside school, and your offences against children?

- To what extent do you see a link between your relationship with any significant adult(s) outside your immediate childhood family and your offences against children?

The data collected in relation to these 3 questions are presented in the section which follows. 


\section{Making the link}

As already noted, evidence as to the extent to which victimization plays a part in later offending is contradictory. It appears that victimisation may be one of several factors which together contribute to sexual offending. In this section of the thesis, the focus is on the men's understanding of their own offending.

Briggs (1995: xiii) posits that CSA is:

'damaging irrespective of whether victims regard it as harmful or 'normal'. Even when men are in jail for repeating the behaviour inflicted upon them, they are rarely aware of the connection between their past learning and their present predicament'.

In contrast with Briggs’ supposition, 17 men identified links between their childhood experiences and later offending. However, only around half (9) provided some detail on those. These data are included later in this section of the thesis.

In an attempt to determine awareness of when sexual attraction to children emerged - and whether perhaps offenders could be classified as either fixated or regressed - the men were asked:

- How old were you when you first became aware that you were sexually attracted to children?

Table 7.1 details the age at which offenders first became aware that they were sexually attracted to children. 
Table 7.1: Age sexually attracted to children?

$(\mathrm{N}=32) \quad$ No. offenders

Not sexually attracted to children 4

$0-16$ years 7

17-19 years 2

20-30 years 7

31-45 years 6

$46-55$ years 2

56-65 years 2

Information missing 2

The majority of offenders (26) identified the age at which they became aware that they were sexually attracted to children. In 7 cases, this was before they reached 16 . Seven men indicated that their sexual attraction to children became evident between the age of 20 and 30; and 8 when they were aged 31 to 45 . For 4 men that attraction emerged later in life, between the age of 46 and 65 .

In order to determine their understanding of the origins of their sexual attraction to children, offenders were asked:

- How do you think you came to be sexually attracted to children?

And as a means of eliciting more specific data on that sexual attraction:

- In what ways did you find children sexually attractive?

Offenders' responses to these questions are presented below.

Around half (9) of the 17 men who identified links between their childhood experiences and later offending provided some detail on those. Fergus recalled the sense of isolation he felt as a child and his inability to interact with his peers: 
'I have always seen a very strong link between childhood experiences and my behaviour in adult life. I was very isolated as a child. My mother and father did not mix very well. So, as a child I was never socialised. I was never playing with other children. When I went to school I was very isolated, I didn’t know how to interact socially'.

He reflected on his attraction to the physical attributes of young boys. This attraction, he felt, stemmed in part from the abuse he experienced; and also from the sense in which he felt different to his peers:

'It has to be something to do with the genitals. Some of that ties in with the experience of being abused at 7 or 8 by the doctor. Also, teasing, being teased as a child about my own body. Being a fat child and a late developer emotionally and physically; being teased in my early teenage years about the size of my genitals. I think that was the roots of an unhealthy fixation - looking at other boys'.

Fergus' account appears to comprise elements of Finkelhor and Browne’s (1985: 355356) concept of traumatic sexualisation, when, following abuse, parts of a victim's 'anatomy become fetishized and given distorted importance and meaning'. Fergus described how his sexual attraction to children emerged at the age of 34 . This was due, in his view, to his fantasies. Being 'surrounded' by children provided him with the 'opportunity' to offend.

Gavin was one of 4 men - out of 5 in total - who, like Fergus, identified a very strong link between his experiences with childhood friends and his later offending. The other 2 men in this group were John and Oliver. Of interest, is that 3 of those men had experienced abuse perpetrated by other young people. Gavin was abused at the age of 12 by a male pupil in the same school year; John and Oliver both experienced sibling abuse, in addition to abuse by other males. Fergus differed, in that he was abused by a professional in a position of trust.

Gavin was also sexually attracted to young boys and became aware of this at the age of 26. Child abuse images are often used by child sexual abusers: 'to fuel and reinforce their child contact activities' (Miller 2013: 516). However, many of those with a sexual interest in children limit their activity to downloading or producing such images, never 
actually engaging in direct contact abuse (Houtepen et al. 2014). Gavin fell into the latter category. He used the Internet 'very frequently' to download indecent images of children. He also communicated with other sexual offenders over the Internet - through chatrooms - collecting and sharing images; and explained how skilled he was in that regard:

'I was able to set a download programme and take everything from a newsgroup while I was out of the house. You could get 20, 30, 40,000 pictures if you wanted to, coming down in one fell swoop'.

Gavin was able to pursue his activities in complete privacy in his own home. His wife was 'not into using computers' so there was no risk of discovery from her. In fact, he recalled that as he built up his collection, he kept many of the downloaded images where they might easily have been discovered:

'I printed out the pictures I liked the most and they were there in folders on the bookshelves’.

Those images were usually of those who 'interested' him: 11 to 13 year old males. Glasser et al. (2001: 492) note that: 'the abuser's target age-group is usually limited to the age when he was himself abused.' Gavin's account appears consistent with this finding. He explained that his attraction to children was:

'invariably to boys the same age as I was when I was being abused. What I thought I was feeling at first was a sort of paternal protectiveness towards them. What had me worried was when I found myself being aroused. By that time, I realised there was something very, very definitely wrong'.

Gavin recalled that he experienced: 'those feelings' regularly. He felt a 'connection' with young boys; and a yearning to regress to the age at which he was abused in order that he could play 'sexual games'. He described the characteristics he found sexually attractive in young boys:

'There were certain physical characteristics that I used to find myself in turmoil about. If there was evidence of boys entering adolescence - they would become greasy around the nose and there would be a little bit of moustache growth - that would tend to make them attractive. There were cases where children were obviously trying to advertise this - their sexual development - by putting up 
messages like: I am developing and I want the world to know. So it was the physical appearance and the body language [that I found attractive].'

As already noted, Gavin had been disgusted and repulsed at the sexual activity he was forced to engage in as a child. As a consequence, he saw his offending as 'therapeutic':

'I was so traumatised by being forced by someone close to my own age to indulge in sexual activities, I wanted to have access to pictures of similar youngsters who are doing the same but enjoying it. My thinking was [when I accessed the images], that it [my abuse] was only traumatic because of my feelings towards it. Look at these [children in the images]; they are thoroughly enjoying it'.

For Gavin, the often smiling images of children being abused depicted enjoyment (Itzin 2000): the polar opposite of his experience of sexual activity as a child. Of course, such images are often directed to achieve such a result. Of interest here, is the way in which the power inherent in IIOC is illustrated: that is, their contribution to the justification and minimisation of abuse (Quayle and Taylor 2002).

Dan reported a 'very strong link' between family experiences and his later offending. Dan had been aware that he was sexually attracted to children for some time before he offended. Being around children took Dan back to his own childhood and the associated sense of 'happiness':

'I first found myself thinking or wondering or staring approximately 4 or 5 years ago [when in his late 20s]. I was in a relationship where children were involved and I enjoyed being with a couple of children. Relaxed, happiness, the laughter [is what I enjoyed]. It made you feel younger and if you were playing with children you were a child yourself. You'd stepped out of the role of being an adult. I knew I had a problem but I hadn't done anything physical at all. Probably about 12 months before the offences, that's when I knew because I found myself looking for the opportunity [to abuse]'.

Dan described how his sexual attraction to children was based on attributes such as: 'the texture, the softness of the skin'. Childhood memories of his sexual activity with his female friend at the age of 6 , were central to his offending:

'The comfort, the happiness. Those memories sliding back and obviously with playing with children and spending time with children those memories crept in which led to my offending'. 
Clive, John, Adam and Oliver had each been abused while in care. Clive reported a 'very strong link' between his relationship with significant adults and his later offending. He was sexually attracted to boys and became aware of that at the age of 28. He explained that he was particularly attracted to the physical attributes of his victims. Once more, fantasy features heavily in Clive’s offending:

'The fall of the body. It's the physical body. You get to the body and then you start to fantasise what you can do with the body. It is letting those fantasies take over is where the trouble is - from fantasy to reality. Because I have learned since I have been here that fantasy can't hurt you, it's what you do with the fantasy that hurts. Masturbation in itself is not a crime. It's when you let those fantasies take over it becomes a crime'.

Clive's account of his offending: 'retains features of his childhood abuse' (Howitt 1995:2). Indeed, his offences appear to be a 're-enactment' or 'direct replication' (ibid.: 61) of the abuse he had experienced:

'[I did] the same as what was done to me, masturbation and things like that' In his view, his sexual attraction to children stemmed from the fact that he was: 'living in [his] past'. Clive explained that the attraction was not towards the child. Rather, the child becomes the conduit by which his own abuse - and the 'same feelings' it evoked - is relived:

'It is not actually the child that's attractive, because when you are doing it you are sort of living backwards. You are doing it to a child but you are illustrating in your own mind what was being done to you. You are sort of reliving the past. You are getting the same feelings as you had then [when I was abused]. You can't say enjoy it but I have got to find a word between enjoy and tolerate - it's somewhere in between them two'.

Adam and John also reported 'very strong links' between their family experiences and later offending. John also identified very strong links between his relationship with childhood friends; and Adam his relationship with significant adults and offending. Like Clive, both had spent much of their childhoods in care. Adam recognised that he was sexually attracted to children at the age of 65. Some years prior to that, however, he had sexually abused his son. He later abused his 2 grandsons and their 3 friends. In Adam's 
case - as with Fergus - the isolation he experienced as a child comes through in his narrative; this sense of being alone, in his view, played a part in his offending:

'I’ve never had a home - I was an orphan. I never had a mother or father, no-one to turn to. At school, there was bullying and I was very much a loner'.

Seven of the 32 men in this sub-sample reported that they became aware of their sexual attraction to children before the age of 16 (Bob, Edward, John, Oliver, Mark, Carl and Donald). All had previously been convicted of sexual offences against children; and 3 of those men - Edward, Mark and Oliver - could be defined as fixated offenders.

Evidence suggests that young people who engage in sexually harmful behaviour (SHB) are not likely to 'specialize' in sexual offending and to perpetrate sexual abuse as adults (Caldwell 2007: 108). Rather, they are more likely to be subsequently convicted of nonsexual offences. This does not appear to hold true for Donald. Donald became sexually attracted to children from a young age and explained that he had first abused his sister at the age of 12 :

'The abuse started within the family. I abused one of my sisters - I was 12 and she was 10 . Then I exposed myself for a number of years. I got caught at 19 . Then, I became more confident in my offending behaviour'.

Donald described how he had been offending: 'all my life: indecent exposure'. He recalled how he would expose himself to children: 'of both sexes...from 6 up to 14, 15 [years of age]'. Interestingly, most recently, he had offended against his 3 sons.

Four of the 7 men referred to above, (Bob, Edward, John and Oliver), provided some detail on the links between childhood experiences and their later offending; and their sexual attraction to children.

Bob reported a 'very strong link' between his childhood family experiences and later offending. Bob had been sexually attracted to children 'as a child from the age of 11 '. He talked of normalising the 'painful' abuse he experienced from a neighbour; and believed 
that his attitude towards it played a part in his later offending. For Bob, tolerating the abuse was a means of receiving care and attention 'from someone':

'The sense of normality of the abuse I experienced, I carried through the whole of my life as a means of care and attention from someone. I don't think my abuse aided me as an abuser, but my attitude towards it had a big effect'.

Edward reported a 'fairly strong link' between his childhood family experiences and later offending. Like Fergus and Bob, he was also sexually attracted to young boys. Edward believed his sexual attraction to children had begun in his 'middle teenage years' when he was 15; and that it stemmed from his own abuse.

John also became aware of his sexual attraction to children at 15. That attraction, he felt, was based on his need to care for someone. Like Clive, John found it difficult to 'deal with' his own abuse. His victims provided some 'comfort' as he sought to do so:

'I used to get comfort from kids because it smoothes my past out. I have got to admit I am fooling myself by offending on other kids because I can't deal with my abuse'.

Like Fergus, Oliver reported 'very strong links' with family experiences; childhood relationships; and also relationships with significant adults and his later offending. His sexual attraction to children began when he was 14 . This stemmed from their vulnerability and small stature which - he explained - made him feel more powerful; and counteracted the sense of powerlessness he experienced as a victim himself (Finkelhor and Browne 1985).

In this section of the thesis, attention has focused on the men's understanding of: the links between childhood experiences and their later offending; how they became sexually attracted to children, and at what age; and why they found children sexually attractive. Next, the emphasis is on their accounts of 'becoming an abuser', beginning with their accounts of how they identified vulnerability in their victim/s. 


\section{Identifying victims}

Bennett and O'Donohue (2014: 957) highlight the importance of 'understanding the process' of CSA to the preventive and treatment agenda. There is a 'lack of consensus' (ibid.), however, as to the mechanics of this process. Thus, here, in an attempt to further understanding, the emphasis is on some of the men's accounts of one element of that 'process': how they identified their victims; and in some cases, the extent to which they drew on their own sexual victimization to assist with that. (None of the men in the other 2 sub-samples provided any detail on this).

Luke, Donald and Robert abused on an intra-familial level; and Bob on both an intra-and extra-familial level, and their accounts are presented first.

Luke had been convicted of incest against one of his daughters. He explained how he had initially been drawn to his victim because of their shared vulnerability:

'I was never good at mixing. I always had difficulty in spelling and things like that at school. She [victim] had got the same problems. When she told me, when she talked to me about her problems at school it was like a mirror image to what I had'.

Donald had offended against his 3 sons, (having previously offended against his sister and also unknown male victims). His approach to his most recent victims was strategic; and he explained that he created a situation whereby he could access children to abuse with ease:

'I know I was intending to get married to have children to abuse'.

Perhaps this decision was based on his assessment that 'selecting' his own children as victims was 'easy'; as perhaps evidenced by his father's abuse of him as a young child. Identifying unknown victims 'outside' on the other hand, was 'more difficult' because of the presence of external inhibitors such as protective adults (Finkelhor 1984). 
Robert's narrative sheds some light on the process he engaged in when identifying his victims. He had offended against 4 of his 6 children (3 of his daughters, and one of his sons). Interestingly, he did not abuse 2 of his sons. Perhaps Finkelhor's (1984: 60) concept of the 'front of invulnerability' played some part in that. The author asserts that:

'Much abuse is undoubtedly short-circuited without the child's knowing anything about it because a potential abuser chooses not to approach that particular child, but goes on to another. This even occurs within a family, where some children will be molested by a father while others are left alone. Abusers undoubtedly sense that some children will not make good targets'.

Although children with a disability appear to be more vulnerable to CSA (Brown et al. 2016), Robert explained why he had not abused one of his sons:

'Well, X being handicapped, he had enough problems of his own, I suppose. Not only that, he didn't keep himself clean when he went to the toilet and that would have put me off'.

It appears that his older son was not a 'good target' (Finkelhor 1984) because of his age and stature.

Bob abused on both and intra-and extra-familial level. His victims were distinguished by a 'need for attention':

'All 3 were in need of attention. They were lonely and wanting the attention of an adult, and that gave me the opportunity to get close to them. With my son, he wanted my attention and to be close to me and that made him vulnerable to abuse.'

There are some parallels here with Bob’s account of the CSA he experienced. He had a poor relationship with his father, from whom he received little attention. He was revictimised in childhood; and valued the attention he received from his first abuser.

Edward, John, Oliver and Dan abused on an extra-familial level and their accounts follow. Having the capacity to identify vulnerability in victims and their families was central to Edward's (and also Dan's) offending. In the case of each of Edward's victims, 
he made use of the father's absence and became a family friend. Of interest, is that Edward had himself been abused by a friend of the family:

'The parents were single - it was just the mum. I used the boys' families to get closer to the boys. Doing all the odd jobs around the house or helping out money wise; giving them lifts; offering to babysit; just getting in their goods books really - getting their trust'.

He explained that one of his victims had 'emotional problems', and that rendered him particularly vulnerable to abuse:

'He had emotional problems and I was using that to get closer to him. His mother found it difficult to control him. I went up there [to do a job] and X [victim] was playing up, so I asked him to help me. And then I started taking him out when I was doing other odds and ends, job wise'.

Edward's approach was discerning, in that he did not offend against all the boys he knew. Here, he describes how he selected his victims based on their response to him:

'I selected them in a sense because I knew a lot of other kids and I never tried it with them. So, I think it was the way they reacted to me whether or not I took it further.'

Several offenders in Briggs’ (1995: 116) sample talked of the ease with which abusers who are often victims themselves - recognise victims who have previously been abused, and are 'sexually and emotionally damaged'. Sadness, isolation, poor self-esteem and indicators of early sexual experience alert abusers to the vulnerable child. There are parallels here with Edward's account and his description of his youngest victim, a nine year old boy, as being 'streetwise' and 'open' to abuse:

'I know one of them was very open. He liked good eye contact, physical contact, and I found out not long after I started to abuse him that he had been abused before. So I think I sensed that in him somehow. He didn't mind the contact. He was very willing to have the cuddle whereas some boys wouldn't do that sort of thing, not even jokingly. I found out that the boy was abused before [through playfighting] and he was very susceptible to being abused again. I was doing more and more risky things, touching him. A boy that wouldn't have been susceptible wouldn't have allowed me to have done it'. 
Like Edward, John was clear as to how he identified his victims. Again, the characteristics of the child and his family played a part in that selection. Once again, there are some parallels between John's childhood - which was characterised by poverty and a lack of love and care - and that of his victims:

'I looked for a child who has got nothing - he ain't got much money; the kids are rogues; they wander about the streets. That sort of kid. I felt sorry for them because they had nothing. I used to think if I get them this, get them that, they will let me do what I want. They were an easy target. Then I would get their trust and I would try and get one parent families because I found it easier and I would offer to take the parents out for the day'.

Oliver explained how he would target unknown male victims in the town centre who met the criteria he set for his victims. His confidence in his approach comes through clearly in his narrative:

'[They had to be] clean, short hair. They had to be shy. I wouldn't go to somebody who was rowdy. I could pick any lad up I wanted to, no problem at all. I never frightened any of them. I had ways and means of doing it. I would go and talk about football and that's the first thing they are interested in. I'd coach them into my way of thinking and get them to come with me'.

Oliver explained how his own experience of CSA assisted with his offending:

' $[\mathrm{By}]$ just getting myself round kids. I knew where they would be, I was more street wise. I knew where people ran away from children's homes because I had been there myself. And I took them somewhere quiet - every offence'.

Dan had first offended against his partner's 6 year old daughter within 2 weeks of moving in with the family. The family were already vulnerable; he recognised that, and used it to his advantage.'Indirect victimization', where a child is affected by the victimisation experienced by a family member, and feels vulnerable and powerless as a result, increases the risk of CSA (Boney-McCoy and Finkelhor 1995); it appears to feature here:

'Child abuse is a very big thing in the mother's life. She'd been abused herself; the older daughter had been abused; her niece had been abused. There's 7 cases of child abuse within that mother's very close family'. 
Dan provided some detail as to how the family's vulnerability assisted with the maintenance of his victim's silence:

'[She'd feel] embarrassed, frightened that she'll be told off for what she is doing. She would also feel frightened that it will split up this family because myself and the mother were showing an awful lot of affection to each other and I was bringing the mother out of her shell. The mother was actually looking better, feeling better and had some pride in herself again and it showed through the children as well. So, obviously there's that pressure upon her, knowing it [finding out about the abuse] would destroy her mother'.

In the next section of this Chapter, some of the men's narratives are drawn on in an attempt to further 'understand' and interpret their accounts of their offending.

\section{Becoming an abuser - further explored}

Here, the narratives of 23 out of 32 men are drawn on in an attempt to further 'understand' and interpret their accounts of their offending. A number of frameworks are imposed on their narratives, including: Finkelhor's (1984: 37) four factors which contribute to the 'making of a child molester'. Moreover, in a further attempt to detect patterns in the men's accounts, they are ordered according to the presence of Core ITs (Ward and Keenan 1999). (A summary of both these models/theories was provided in Chapter 5, where the theoretical underpinnings to the research are outlined).

Ward and Keenan (1999: 836) stress the importance of investigating: 'the nature of sexual offenders' beliefs about their victims, and people in general, and to develop detailed descriptions of their core ideas'. (p.836). They argue that:

'an implicit theory perspective can provide us with a useful framework within which to approach the complex problems of child sexual abuse and offender treatment' (p.836).

The data drawn on during this section of the thesis were analysed from an IT perspective in an attempt to understand that complexity, and make some contribution to more effective intervention. 
As previously noted, the 5 ITs relevant to child sexual abusers' thinking are:

\author{
children as sexual objects \\ entitlement \\ dangerous world \\ uncontrollability \\ nature of harm
}

Table 7.2 summarises the extent to which the 5 ITs were present in the men's narratives. The majority of the men (17 out of 23) clearly viewed 'Children as Sexual Objects'. Fifteen referred to a sense of 'Uncontrollability' inherent in their offending. For 11 men, the world is a 'dangerous' place. There was evidence of 'Entitlement' in 4 men's narratives; and 'Nature of Harm' in 3 accounts. 
Table 7.2: Core ITs evident in narrative?

$(\mathrm{N}=23)$

OFFENDER

CORE IT

\begin{tabular}{|l|l|l|l|l|l|}
\hline & $\begin{array}{l}\text { Children as } \\
\text { Sexual } \\
\text { Objects }\end{array}$ & Uncontrollability & $\begin{array}{l}\text { Dangerous } \\
\text { World }\end{array}$ & Entitlement & $\begin{array}{l}\text { Nature of } \\
\text { Harm }\end{array}$ \\
\hline EDWARD & $\mathrm{Y}$ & $\mathrm{N}$ & $\mathrm{Y}$ & $\mathrm{N}$ & $\mathrm{Y}$ \\
\hline JOHN & $\mathrm{Y}$ & $\mathrm{Y}$ & $\mathrm{Y}$ & $\mathrm{N}$ & $\mathrm{N}$ \\
\hline OLIVER & $\mathrm{Y}$ & $\mathrm{Y}$ & $\mathrm{Y}$ & $\mathrm{Y}$ & $\mathrm{N}$ \\
\hline CLIVE & $\mathrm{Y}$ & $\mathrm{Y}$ & $\mathrm{Y}$ & $\mathrm{N}$ & $\mathrm{N}$ \\
\hline HARRY & $\mathrm{Y}$ & $\mathrm{Y}$ & $\mathrm{N}$ & $\mathrm{N}$ & $\mathrm{N}$ \\
\hline BOB & $\mathrm{Y}$ & $\mathrm{Y}$ & $\mathrm{N}$ & $\mathrm{N}$ & $\mathrm{N}$ \\
\hline ADAM & $\mathrm{Y}$ & $\mathrm{Y}$ & $\mathrm{Y}$ & $\mathrm{N}$ & $\mathrm{N}$ \\
\hline FERGUS & $\mathrm{Y}$ & $\mathrm{N}$ & $\mathrm{Y}$ & $\mathrm{N}$ & $\mathrm{N}$ \\
\hline LUKE & $\mathrm{Y}$ & $\mathrm{Y}$ & $\mathrm{Y}$ & $\mathrm{N}$ & $\mathrm{N}$ \\
\hline BRIAN & $\mathrm{Y}$ & $\mathrm{Y}$ & $\mathrm{N}$ & $\mathrm{N}$ & $\mathrm{N}$ \\
\hline BARRY & $\mathrm{Y}$ & $\mathrm{N}$ & $\mathrm{N}$ & $\mathrm{N}$ & $\mathrm{N}$ \\
\hline NEIL & $\mathrm{Y}$ & $\mathrm{Y}$ & $\mathrm{N}$ & $\mathrm{N}$ & $\mathrm{N}$ \\
\hline ARTHUR & $\mathrm{Y}$ & $\mathrm{N}$ & $\mathrm{Y}$ & $\mathrm{N}$ & $\mathrm{N}$ \\
\hline ROBERT & $\mathrm{Y}$ & $\mathrm{Y}$ & $\mathrm{N}$ & $\mathrm{Y}$ & $\mathrm{Y}$ \\
\hline WILLIAM & $\mathrm{Y}$ & $\mathrm{N}$ & $\mathrm{N}$ & $\mathrm{Y}$ & $\mathrm{N}$ \\
\hline DONALD & $\mathrm{Y}$ & $\mathrm{Y}$ & $\mathrm{N}$ & $\mathrm{Y}$ & $\mathrm{N}$ \\
\hline CARL & $\mathrm{Y}$ & $\mathrm{N}$ & $\mathrm{Y}$ & $\mathrm{N}$ & $\mathrm{N}$ \\
\hline KEN & $\mathrm{N}$ & $\mathrm{Y}$ & $\mathrm{N}$ & $\mathrm{N}$ & $\mathrm{Y}$ \\
\hline ELLIS & $\mathrm{N}$ & $\mathrm{Y}$ & $\mathrm{N}$ & $\mathrm{N}$ & $\mathrm{N}$ \\
\hline DAN & $\mathrm{N}$ & $\mathrm{Y}$ & $\mathrm{N}$ & $\mathrm{N}$ \\
\hline FRANCIS & $\mathrm{N}$ & $\mathrm{Y}$ & $\mathrm{N}$ & $\mathrm{N}$ & $\mathrm{N}$ \\
\hline CHRIS & $\mathrm{N}$ & $\mathrm{N}$ & $\mathrm{Y}$ & $\mathrm{N}$ & $\mathrm{N}$ \\
\hline DYLAN & $\mathrm{N}$ & $\mathrm{N}$ & $\mathrm{N}$ & $\mathrm{N}$ \\
\hline
\end{tabular}

In the section which follows, the presence of Core ITs in the men's accounts are explored, beginning with the extent to which it is evident that they perceive 'Children as Sexual Objects'.

\section{CHILDREN AS SEXUAL OBJECTS}

Reference to 'Children as Sexual Objects' was evident in around two thirds (17) of the 23 men whose narratives are examined here. Some men shared some characteristics and 
could be defined as fixated offenders: namely, Edward and Oliver. On other levels, however, the men differed: Edward, John and Oliver abused purely on an extra-familial level; Donald offended against his own sons; and Bob offended against both intra- and extra-familial victims. Again, distinctions are made between the men and the form their offending took, in order to tease out difference, during the course of analysis.

\section{Perpetrating intra-familial CSA}

Several of the men who perpetrated intra-familial abuse clearly viewed children as sexual objects. Luke, Brian, Barry, Neil, Arthur, Robert, William and Donald fell into this category. Luke had been convicted of incest against his daughter. His marriage had broken down; and his wife had left the family home leaving him to care for their 2 daughters. He described how he viewed his victim as an adult:

'I don't think I looked on X as a child. I think [it was] because she was doing the housework, the cooking, all that.'

He believed that his daughter had initiated the sexual activity:

'The first time the offer was from her. She offered first by telling me: if that's what you want, then that's all right, as long as you don't hurt me'.

Brian had been convicted of indecent assault against his stepdaughter. He believed that his victim had a 'crush' on him:

'She was always cuddling up. She was always with me and she had a crush on me and I took advantage of her having a crush on me'.

He described how he 'cheated' on his wife with his victim:

'I was going through a period where I was cheating on my wife with just anybody. And my stepdaughter was one of them. At the age of 13 she looked like someone 17,18 . She had a body built like a well-developed young lady'.

Barry had also offended against his stepdaughter and believed that his victim 'knew what she wanted' and was partly to blame for the abuse: 
'I didn't do anything. She had her eyes open. She knew what she was after. I didn't sexually abuse her. She knew what she wanted.'

Neil had offended against his granddaughter, who in his view, had knowledge of sexual activity prior to the abuse:

'The first time it happened I said to her lock the door. And the second time, she come in and she said: "I have locked the door". Just the same thing [happened] trying to have intercourse with her'.

He talked of how his victim took the initiative; and placed responsibility on his granddaughter, insisting that 'she should have kept away':

'After the first time she was egging me on. [She wasn't responsible] the first time but the times after that she was. She can act dull but she ain't dull and why was she coming here? And you know, it is right for a granddaughter to come and see me, but after what happened the first time she should have kept away'.

Neil neither believed that he had harmed his victim, nor that the abuse had affected her in any way: 'No, I never hurted her’.

Arthur had abused his niece. Again, there is an emphasis on the child initiating abuse and 'volunteering' for sexual activity:

'The first offence was at my sister's home. I was living there at the time. It happened several times over a 6 month period. I was babysitting in my sister's home - I was 22. I was watching a sexy film and I had an erection. My niece came in, asked what it was - erect penis - and I showed her. With the second offence, my niece came down and asked me if she could touch it. My niece came to my bedroom and the offences happened. I never actually did anything to her. She was volunteering. She asked and I let her [touch me]. It became easy because she didn't tell anyone. She kept coming to me'.

Robert offended against 4 of his 6 children (3 of his daughters and one son). He talked of having a ‘special attraction' to his eldest daughter and a 'special relationship' with her:

'I had strong, not only sexual feelings, but I felt I loved her more than I did my wife. And I probably still do. We had a very close relationship despite the abuse because she didn't seem to mind that. While my wife was so ill, with the breakdown and that, she was the one I could talk to...the one I could get to help me to organise the rest of the family'. 
He believed that his sexual attraction for his eldest daughter stemmed from bathing her:

'I was bathing her and she looked sexually attractive because she was naked. First of all it was just touching. Then it led on to other things. I was sexually aroused because I hadn't had a relation with my wife over that period of time. We used to go years without sex, especially when she first had an aversion to it...The opportunity [to abuse] was there, seeing her naked, one thing led to another. I think that was the case with the others as well'.

He believed that his eldest daughter 'didn't mind' about the abuse and in time, initiated the sexual activity, appearing 'willing':

'She used it - sex - to stay home from school, because she didn't like school. She would say I don't want to go to school; and that gave me the hint that if I let her stay home, sex would be on the cards. It was a sort of 2 way thing - she didn't seem to mind too much about the sex. The others did but she didn't. She was usually approaching me after a while. She just seemed to be willing'.

William had abused his own son and 2 stepsons. In the first instance, like Robert, William developed a sexual attraction to his son while bathing him. Again, implicit in William's narrative - as in Luke's - is the sense in which his wife was 'absent' from their relationship in some way (Finkelhor 1984):

'My first wife was ill - she had a bad heart and had an operation. I used to get X ready for school and bath him and that's when I started abusing him - it was when he was in the bath. It was when I was soaping him down in the bath - that's when it started'.

He believed that one of his stepsons enjoyed the abuse:

'X was quite willing you know, because he didn’t complain. He used to take part in it quite willingly'.

He also believed his youngest victim - his son - enjoyed the abuse:

'I didn't order Y about. My first wife slept downstairs because she was ill and Y used to sleep in my bed and I used to think, well, he doesn't mind being sexually abused; he is enjoying it, he wouldn't be in my bed otherwise'.

Donald had abused his 3 sons and believed that he was showing his victims love:

'I never spoke to the victim about what I wanted him to do. I strongly believed I was showing my son love. I was thinking that my son was enjoying it because he 
had an erection. I never got my son to take part in arousing me. I thought I'd frighten him or upset him if I got him to masturbate me'.

\section{Perpetrating extra-familial CSA}

Edward had abused 3 male victims. He was a friend of each of his victims' families and explained: 'I befriended families, more or less purposely [to abuse]'. He had moved in with his first victim's family (as a lodger) with the express purpose of committing CSA. He described his approach as 'very calculated'. With his second victim he recalled:

'I just liked him sexually and I just knew that I wanted to get to know his family so that I could get closer to him’.

John recalled that when he was first convicted of sexual offending he did not believe that sexual activity with children was wrong or would be harmful to them. His only concern was to gain sexual gratification. There was no 'sympathy' for his first victim, or any thought as to the cost of abuse for such a young child:

'When I first got into trouble, abusing a 7 year old, I didn't even know it was wrong. I had no sympathy for my victim'.

Oliver offended against victims who were unknown to him. Like John, Oliver believed that it was: 'normal to have sex with children'.

Clive emphasised the caring, close nature of his relationship with his victim, which in his view, was like that of father and son. However, the sexual nature of that relationship; and the sense in which the child was a sexual object used by Clive in the pursuit of sexual gratification, was also evident:

'Me and [victim] were like father and son. I thought the world of him and basically we were just fondling in bed. Then I took it a bit further and then I sucked his willy; and the real trouble came when I tried to shove a finger up his bottom [at the age of 5]'.

Harry, who had abused his partner's daughter, described his 12 year old victim as: 'quite mature for her age'; and as having 'consented' to the sexual activity: 
'I wouldn't have done it if she hadn't consented to it. I thought, I need a girl and I had known her for a long time and it just happened. Started kissing and it went on from that. But at no time did she say that she didn't want to do it - she just carried on'.

\section{Perpetrating intra- and extra-familial abuse}

Bob described his victims and his interaction with them in sexual terms, and as characterised by 'gentle loving':

'The attraction is connected to a sense of caring and gentle loving. That's what attracts me to the shape and look of a young boy's body. My thing is fondling and stroking their bodies. Thin, blonde haired and blue eyed is what attracts me'.

Adam had offended against his son, grandsons, and his grandson's friends. He believed that all his victims 'were happy' with the abuse. Had they not been, he felt sure they would have spoken out:

'I had this feeling that they [victims] were happy with what was happening and they would honestly speak [out] if I had done any harm to them or hurt them'.

Fergus was one of 2 men who abused as an uncle - the other was Arthur. Fergus' case, however, is particularly interesting as he had offended against 5 male victims, some of whom were related to him. His narrative is permeated by the sense in which children are perceived as sexual objects (and also by the Core IT 'Dangerous World'). Fergus described how he first started abusing his nephew; and placed emphasis on the fact that his nephew 'appeared to like’ the sexual activity:

'It started off with my nephew over a period when he was living with us. His parents were having marital problems and it started with cuddling him, stroking him and moving on to touch his genitals and stimulating him and masturbating him over a period of 3 years, and then moving on to getting him to touch me and masturbate me. With all the other children it was touching their genitals under their clothing but they were the sort of incidents that happened once or twice in each case and then stopped. But with my nephew it was a situation that continued. The other boys didn't appear to want the contact - he appeared to like the contact'. 


\section{UNCONTROLLABILITY}

Finkelhor (1984: 354) notes that as part of the process of abuse: 'the dynamic of powerlessness distorts children's sense of their ability to control their own life'. Uncontrollability featured in 15 of the men's narratives. This was evident on a number of levels. Some of the men's accounts were permeated by the inevitability, in their view, that their childhood sexual victimisation resulted in their becoming an abuser. For others, that uncontrollability took the form of stressors such as relationship difficulties which led them to offend. Yet others, referred to the use of substances and IIOC as diminishing internal inhibitors (Finkelhor 1984), and resulting in offending.

Indecent images of children played a central part in offending for 3 of the men in this sub-sample: Bob, Ken, and Arthur. Bob recalled that at the time of his offences against his son and his friend he had been depressed and angry, and had begun 'using child pornography':

'I became ill with severe chest pains. I went into hospital and was off work for 6 months. I became depressed and felt useless. I was very angry with myself and everyone else. I connected to the Internet and started using child pornography and got hooked to the child porn scene. It developed from fantasy to reality over a 2 year period. The offences were committed at the end of that period'.

For Bob, 'child pornography' was a central feature of his offending:

'It was an important part of my decision chain. It fed a huge amount of distorted thinking. There's lots of people doing this and therefore it can't hurt. That was the way I was thinking at the time.

He reported using the Internet to pursue a sexual interest in children 'very frequently'. He also used the Internet - albeit unsuccessfully - as a means of accessing children to abuse in other countries. He was sure that had he found a 'contact' in Thailand through whom he could have accessed victims, he would have done so:

'Once, through an Internet connection, I went to Thailand (having spoken to someone on the Internet after I'd abused the boys for the first time). I set up a business trip to Thailand but didn't abuse anyone because I was frightened of the situation. I didn't know where to find or take a boy. I was afraid of being caught 
and ending up in a Thai jail. Had I had a contact, there's no doubt in my mind Id have abused boys'.

As in Bob’s case, 'pornography' was a central feature of Ken's offending. He explained how he became reliant on 'pornography', which he regularly accessed over a 7 year period:

'I obtained a magazine about child pornography. I used to look at it and masturbate to it. It was like an awakening. It was a taboo subject. I didn't think there was that kind of material available'.

Some of the other men in this sub-sample, particularly those who had been abused in care, were of the view that their childhood sexual victimisation led to their offending. John, for example, believed that the sexualised nature of his childhood in care led to him 'passing on' his abuse to his victims:

'I was brought up in a children's home. I was brought up with sexual perversions. All my life I have been brought up with sex - being abused; being abused at school. There are kids coming back who have left, coming back and abusing us; teachers abusing us. It's always been abuse and I am carrying out what I went through myself. What I am doing is, I am passing it on to my victims'

Some men (John, Oliver, Donald and Luke) were of the view that they still represented a risk to children. Of note, is that John, Oliver and Donald had completed the SOTP and Luke was undertaking it at the time of interview. The implications of this are further explored later in the thesis. John felt that above all, he needed to understand why he was abused. According to John, learning to cope with what happened to him has proved extremely difficult. He clearly felt that his that a lack of understanding as to what happened to him; and a resulting inability to cope with his own abuse were the factors most likely to contribute to further offending:

'Sex offenders like us, some sort of better help [is needed] to make them admit responsibility and cope with the past, because it's the past what keeps creeping up. It's very hard because you can see what was happening to you but you don't know why and that's the hardest point. Nobody will listen and show us how to cope with it [abuse] - it is very hard. I have spent nearly 39 years trying to cope with mine. I have got to admit I am fooling myself by offending on other kids because I can't deal with my abuse. Nobody will listen. I've been done for abuse 
but we have still got to cope with our own abuse and what happened when we was kids and that is the hardest - that's what makes us give up'.

Oliver believed that he became an abuser because, like John, he 'was brought up in it [abuse]'. Being 'overpowered' as a child led to a desire for power as an adult:

'I got used to it [abuse]. I was conditioned into it. I always believed that I came into care looking for a father that I never had. These people [staff] held their power over me and they was enjoying it. It's got to do with my childhood affection, being overpowered and then me having power [by abusing].'

Oliver's narrative was permeated by his reflection on his own status as a victim. He described how he viewed his offending as 'normal'; and referred to himself as 'damaged':

'I was treating it as normal what I were doing to my victims. It was alright because it happened to me. I've done it and it has happened to me and that's why I have been going out and doing it. I am doing it because I was damaged'.

He described how difficult it was to stop abusing and to 'get rid' of his fantasies:

'You couldn't stop it [the abuse]. You knew it was wrong and you knew you couldn't get rid of your fantasies, so you carried out your fantasies. When I was abusing other lads I was thinking about me being abused. I used to lie on my bed and wait for somebody to come in and abuse me. I felt dirty'.

As already noted, although Oliver had completed the SOTP, he still had reservations as to how safe he would be around children. He recognised the part played in this by his normalisation of his own abuse:

'I sometimes still think it is normal. I know it is wrong but sometimes I think I will go out there and I will do it again'.

For Clive, leaving care without having dealt with the abuse he experienced, proved disastrous; and meant that he was equipped with the 'instructions' as to how to abuse:

'Leaving care is like being thrown out of one situation into a new situation and having to do the best with it that you can because you have got no way of actually knowing what is going to happen next. One day you are a child and the next day you are fully grown up. You've got no way of going back, so you have got to push your way forward into a life that you got to try and understand too quick. I 
knew nothing basically of the outside world. You are left with all these feelings [about the abuse] that you can't let go of. That's where the trouble starts - you have left school with the instructions of how to offend'.

Clive realised he needed help but was also aware of his competing sexual needs:

'I can remember actually saying to myself I need help for this, but how the hell do you get help? The thing is, on the one hand I wanted help but on the other hand I didn't want to lose $\mathrm{X}$ and the one always competed against the other'.

Ellis had been raped by his uncle in childhood. He felt that the physical pain he experienced when he was abused led to his offending:

'Because of what happened to me [I became an abuser] - the pain I went through'.

Abuse may be enabled where family members share a room or bed (Finkelhor 1984). Dan described the fact that his victim slept in the same bed as he and his partner as a 'temptation'. Ready access to his victim and the lack of external inhibitors (Finkelhor 1984), meant that abusing was 'too easy' in this case:

'It's just the fact that it's [an opportunity to abuse victim] there beside me and it's there all the time. The temptation was too great and I weakened. Once I'd done it once, I built the courage up to do it the once, then after that it just went straight downhill. It was too easy'.

A number of men were of the view that difficulties with relationships contributed to their offending. Luke's narrative, for example, is permeated by a sense of 'Uncontrollability'. He had offended against his daughter and in this case, there is an emphasis upon: 'factors beyond an offender's control that underlie his sexually abusive behaviour' (Ward and Keenan 1999: 831). Luke believed that marital problems led to his offending. He recalled: ‘if my marriage hadn’t broken down I would never have gone down this path'.

Glasser et al. (2001: 492) note, that in cases of incest:

'it is not uncommon for generational boundaries to be eroded by the father, who takes his daughter as a replacement for his wife at an emotional level before he uses his daughter sexually'.

There are some parallels here with Luke’s description of his abuse: 
'The family have been through a lot of hardship. The wife and I up to this time was perfectly happily married, nice home and [we lost several members of our family]. It was very upsetting. My wife took to alcoholism. She was drunk all the while. She left me with [my daughters]. X was about 8 and A was about 20 months old. I had to give up my job. X - that's the one I offended against seemed to take on more responsibilities at home. She ruled me and I let her. I let her take charge of the house and she took the place of her mother. I let her do it, I could have stopped it obviously, but I didn't. By this time I had got a full time job again so her help was very important to keep the family together, because I couldn't cope with it all myself.

Things started to happen then that I wish had never done [the abuse]. She said: "it is alright as long as you don't hurt me” and I didn't hurt her. I was exceedingly gentle with her. And she said, the first time we must have had sex the proper way: "if I say you are hurting me will you stop?" And I said: "Yes, I will”. Things started to develop from there'.

Luke was undertaking the SOTP at the time of interview. His belief that his victim - his daughter - had 'led him on' clearly persisted and comes through in his narrative. Luke was of the view that understanding offending on an individual level, in particular, previous childhood sexual victimisation and the part played by that in subsequent offending, is a crucial element in desistence:

'Prison staff should listen to us more instead of trying to put them [sex offenders] in the same pigeon-hole, all of them. They [sex offenders] are all different; they are not all the same. There is a reason why you do everything. I think they should look for the reasons why it happened.'

Brian explained that his offending against his stepdaughter stemmed from the fact that he was denied a full sexual relationship with his wife because of her disability:

'My wife was completely disabled so sexual activities with my wife was very rare or nil. Only partial sex could be had'.

Robert described how, prior to his offending, his family had been experiencing difficulties. He had been in poor health before the birth of a son with disabilities. His wife's mental health problems, following the birth, were exacerbated by financial difficulties. Moreover, he explained how his wife's childhood sexual victimisation meant that: 'she wasn't very keen on sex but she did perform it as a duty'. Robert talked of the 'conditions being right' for abuse: 
'At that time I started offending. I was very, very depressed all through that period because of the strain. I was ill and I think that led to the assault [on his older daughter]. I don't think it would have happened without that. I'm not saying that maybe I haven't got a tendency that way but I don't think I would have given in so easily if it wasn't for the conditions being right for it at that time. In my case it was the opportunity, my own feelings, my emotional needs. If my sexual relationship with my wife had been good I don't think it would have happened'.

Adam and his wife had also experienced difficulties with their relationship. They had one child with serious health problems. That child needed constant care and attention; they began sleeping in separate bedrooms and according to Adam, 'grew apart'. Sexual activity with his wife ceased and at that point, Adam began abusing one of his sons who was aged 8. He explained:

'If only I'd have had love, the kind of love, the sexual love I wanted [from my wife] it never would have happened. The sexual part of my life was never good'.

Donald recognised that nothing could have stopped him abusing:

'I felt then [when I was offending] that I was showing my victims love. I don't believe anything could have stopped me at the time because of my way of thinking.

Donald, who had completed the SOTP, was also aware of the risk that he would reoffend:

'I have to recognise as a perpetrator that I'm capable of committing an offence again. It would be very rigid of me not to think so'.

Three men (Neil, Harry and Francis) talked of the part played by substance abuse in their offending; and in breaking down internal inhibitors (Finkelhor 1984). According to Neil, his reliance on alcohol and drugs led to his offending against his granddaughter.

Harry described how, at the time he offended against his partner's daughter, he: 'was doing a lot of drugs' and was also drinking heavily.

Francis had abused both his daughter and son. He, too, believed that his offending stemmed from his substance misuse: 
'I put it down to the amphetamines I was taking. I was having a bottle of vodka a day plus super glue. I was spending about $£ 40$ a week on amphetamines, street stuff...I wasn't $100 \%$ in my head with all the stuff I was taking'.

\section{DANGEROUS WORLD}

Here, the emphasis is on 'the world as a dangerous place' (p.829) where others pursue their own interests, exhibiting behaviour which is rejecting and abusive as they do so. Ward and Keenan (1999: 829) draw attention to the two strands evident within this theory. First, the need to:

'fight back and achieve dominance and control over other people. This involves punishing individuals who appear to inflict harm on the offender and. especially, to ensure that his own position is strengthened. Therefore, if children and/or women are perceived as threats and in need of retribution, they may become victims of sexual abuse'.

This Core IT was evident in 11 of the men's narratives. Carl's account provides an illustration of such thinking. He explained that he found it difficult to cope with the fact that his wife had had previous relationships with men (prior to their marriage) and he saw his sexual abuse of their daughter as 'payback':

'Even when I was sexually abusing X [victim] I used to justify it to myself that it was payback. I was thinking, one day A [wife] is going to know about this, so this is like retribution'.

Chris and Oliver had both been abused while in residential care and their accounts were permeated by a sense of anger. Like Carl, both saw the abuse they perpetrated as some form of 'payback'. Chris made clear that he abused out of 'anger and spite':

'In temper and hatred you'd be surprised at what you can do. Mine [abuse] was done through anger and spite. All I wanted was payback’.

For Oliver, abusing his victims was a way of 'getting back at society'. He clearly felt that those who could - and should - have protected him, instead, let him down badly:

'Now I am getting my own back at society. It [abuse] happened to me and they [abusers] got away with it. Social Services - they put you in care; then they made 
me get abused. They took my family away from me; they locked me up - so why can I not go out and do it [abuse]? That's the way I was thinking'.

'Blockage' (Finkelhor 1984: 43) appears to feature in Arthur's account. He recalled how he had been bullied by his sister at home, and then at school:

'I was very small. I was teased about my lack of knowledge about sexual reproduction. I was called gay because I'd never had a girlfriend'.

His first sexual experience had not been positive and this led to him blaming his victim:

'At 18, I started swearing, drinking and looking at porn magazines. On my first sexual encounter the girl told me I was pathetic and useless. When my niece jumped on my lap, I blamed her'.

The second 'strand' to the IT 'Dangerous World' relates to the: 'unreliability of adults and the dependability of children' (p.829-830). Here, emphasis is placed on adults as rejecting and untrustworthy, and as taking advantage of offenders. Children, on the other hand are perceived as accepting, trustworthy, reliable, loving and caring. Whereas in the first strand, the focus is on achieving dominance and control, here, offenders' believe they are incapable of dominating adults or retaliating against them. Offenders' emotional needs can be satisfied by children who will neither reject nor exploit them. For some of the men in this sub-sample, the adult world is clearly a place where they feel insecure and out of place; and where they sometimes experience rejection. In response, some referred to the need to dominate and exert power.

Donald was particularly drawn to children's 'vulnerability' and innocence, and the sense in which they can be controlled:

'It's knowing that I can have 100\% control over that innocence. Innocence and vulnerability were attractive to me'.

Oliver was also attracted to children's vulnerability and small physical stature; and the fact that he could exert power over them as a result of that:

'they are small, vulnerable, I can do what I want with them'. 
Fergus, recalled his own feelings of inadequacy and explained that he was attracted to children's innocence and powerlessness. Abusing gave him a sense of 'power' too:

'It's my power in the situation [abuse]'.

Being at home, provided Fergus with the opportunity to offend and allowed him to withdraw from the adult world, where he felt insecure. For Fergus, the non-judgemental attitudes of children allowed him to feel 'in control':

'I don't feel this child will be judgemental of me and I can feel confident in this situation - I am in control'.

Several of the men, particularly those who had been abused in residential care: (Oliver, John, Adam and Clive) saw the abuse they perpetrated as meeting an emotional need. In addition to enabling him to occupy a position of power, Oliver recalled that his need for a relationship with children stemmed from the fact that he felt 'lonely'; in need of affection; and was unable to relate to adults. There is a sense of ownership evident here as he refers to his victims as 'my children'. Moreover, there is also evidence of the need to 'control' in Oliver's narrative:

'When I went with the lads [victims] I wanted a relationship. I wanted somebody. I wanted to be with somebody because I was lonely. I have gone out there for affection, because I couldn't talk to adults. I picked lads up for oral sex because I loved it. I love cuddles and I love kissing. I picked my children up because I loved them. I can trust them. I like being around children. I was controlling them - I looked to control, me'.

John also believed that the abuse he perpetrated met an emotional need; and resulted from his need for love and wanting to care for someone:

'I felt like love; things I missed in my own life - care and things like that'.

Adam, too, believed that his victims provided him with the love he missed in a childhood which left him feeling isolated, alone and devoid of affection:

'The love I was getting from them was absolutely great. I wanted love so much myself'. 
Clive described how he found it: 'easier to talk to children than I do adults'. This, he felt was due to his 'fear of adults':

'Because of what went on in care, it leaves you with a fear of adults. You have always got this sort of nagging feeling at the back of your mind that I am going to get knocked about again'.

According to Edward, his offending was 'more of an emotional thing' than sexual. As a child, he had a difficult relationship with his mother who he found to be 'very cold'. He explained that through his offending, he:

'felt wanted. It was the only time I have ever been touched and held'.

Dylan was the only man of the 40 to offend while in a position of trust. He recalled an unhappy childhood and how he 'hated his parents':

'I didn't enjoy my childhood. I used to have a leather belt across my back. We were never a close family. My mother used to rub my face in my faeces and put my head down the toilet. I felt unwanted and had a low opinion of myself. My mother used a leather strap on me regularly. My parents saw it as no more than smacking. I saw it as the end of my world. I hated my parents'.

For Dylan, abusing his victims met an 'emotional need'. He felt that there was an 'emotional bonding' with his victims:

'They gave me something. They wanted me and wanted to spend time with me. I think I needed somebody to want me. I did love these youngsters. Emotionally they meant a great deal to me.'

While professional perpetrators share many characteristics with other child sexual abusers (Moulden et al., 2007), the sophisticated techniques they employ in order to abuse, set them apart (Sullivan and Beech 2002). Gallagher (1998) draws attention to a pathway characterised by explicit planning, for example, grooming. Dylan explained how he 'developed a relationship' with his 'main victim' and how the affection he displayed to Dylan - who had 'never felt wanted or loved' - drove the abuse:

'I knew the main victim from when he was 12 up until 21. The first victim, I developed a relationship with. The last 2 were opportunist - there was rough and tumble and I would touch. X - the main victim - seemed to attach himself to me. 
I've never felt wanted or loved and he showed me affection. I'd take him fishing and this developed into overnight and weekend stays. I'd take him on holidays. He was paralysed from the waist down and had toileting problems - he had a catheter for his bladder and bowel. I helped with that and getting in and out of the bath when he stayed with me. I don't think initially I wanted sexual satisfaction out of it, but I had a desperate need for affection and to be wanted. I took advantage of his need for help with catheterisation and washing him down. I filmed him in the bath when we were away with the intention of arousing myself when at home.'

\section{ENTITLEMENT}

A sense of 'Entitlement' was evident in 4 men's narratives; 3 of those had abused several of their own children. According to Finkelhor and Browne (1985: 357), inherent in the concept of powerlessness, is the sense in which the child's: 'will, wishes, and sense of efficacy are repeatedly overruled and frustrated'. In Oliver's case, the fact that he had no choice but to comply - and accommodate (Summit 1983) - to abuse in childhood resulted in a belief that he was entitled to limit the choice of others in that regard. Thus, he instilled in his victims the sense that they had 'no choice' but to engage in sexual activity with him:

'Well, I had been there [a victim]. I just taught them to feel that they had no choice’.

For Seymour (1998: 417), the 'patriarchal nuclear family’ serves as a source of power for males over females; and functions as an environment in which 'children are viewed as possessions'. Thus, CSA is enabled. This supposition appears of relevance in a number of cases. Robert, for example, offended against 4 of his 6 children and referred to all his victims as being: 'easily available' because they were in his parental care. Thus, he was provided with 'the opportunity' to abuse with 'less likelihood of getting caught'.

William's narrative, too, provides an illustration of how the power associated with parental roles - and also adult physical stature - functions to facilitate and conceal abuse against male victims as well as females (in this case for many years): 
'Then, I was a really big chap and I didn't realise until I heard my voice on tape one day how loud it was and that must have been frightening, especially to a youngster. I didn't say to them don't tell anybody or the both of us will be in trouble. I didn't have to say that, they just did what I asked them, the 3 of them. I suppose I must have looked to them like a monster - I was a lot bigger than what I am now and I used to shout a lot'.

While William believed that 2 of his victims enjoyed the abuse, he recognised that the oldest victim: 'wanted no part with it at all'. William therefore exerted the power inherent in his parental role and:

'took him up to the bedroom and just ordered him to do it'.

Donald had abused his 3 sons and described the importance of parental control to his strategy:

'I thought, it's my son and I was in control. He knew me and trusted me and I could manipulate that trust. It gave me power and control knowing I could do what I wished to. Selecting my own children [as victims] is about parental control - it's easy'.

\section{NATURE OF HARM}

There was evidence of the IT 'Nature of Harm' in 3 of the men's narratives: those of Ken, Edward and Robert. While Ken reported having some inhibitions about the sexual contact he had with children, he reflected on the extent to which he excused his behaviour:

'I said it didn't hurt; boys do it all the time; it's encouraging their sexuality and helping them grow'.

Edward explained how his last victim, a 9 year old boy:

'used to wander the streets a lot so I started telling myself that it is better for him to be with me than wandering the streets where he could get hurt, even though I was the one hurting him'.

Robert described his 'attitude' during the period when he abused 4 out of 6 of his children: 
'What was going through my mind at the time was that they would probably be going to do it [have sex] with their schoolfriends anyway eventually, so what odds'.

\section{Chapter summary}

In this Chapter, the extent to which the men identified links between their own childhood sexual victimisation and their offending was explored; and the presence of Core ITs in their narratives examined, as a means of understanding the belief systems which supported the perpetration of abuse.

Seven men reported that they had developed a sexual attraction to children before they reached the age of 16 . Only 3 of those, however, (Edward, Mark and Oliver) appear to fit the criteria for fixated offenders, those who have: 'from adolescence been sexually attracted primarily or exclusively to significantly younger persons' (Groth and Birnbaum 1978: 176).

Some of the men identified links between their own childhood sexual victimisation and their later offending. The isolation he experienced as a child featured for Fergus; together with his 'unhealthy fixation' for other boys' genitalia following his abuse. Gavin was attracted to boys who were the same age as he was, when he was first abused. The traumatic nature of his abuse by a male peer at school resulted in him viewing his offending - which took the form of accessing IIOC - as 'therapeutic'. Dan explained that his childhood memories of the sexual activity with a female friend at the age of 6 (which had been directed by her older sister) 'led' to his offending.

Clive, John, Adam and Oliver had each been abused in care. Clive appeared to re-enact his abuse in order to relive his past. John sought 'comfort' by abusing his victims, and saw it as a means of dealing with his own abuse. For Oliver, his victims' small stature and vulnerability engendered a sense of power in him which had been lacking in childhood. Like Fergus, John referred to the isolation he experienced as a child and the link between that and his offending. Bob talked of normalizing his abuse and believed 
that his attitude towards it played a part in his offending. Edward described his 'very calculated' approach to identifying victims as stemming from his own abuse.

Some offenders in this sub-sample described how they identified children with specific characteristics who would be vulnerable to abuse. In some cases, they drew on their own experience of CSA to assist with that. Factors which featured here included those associated with the child and their environment: namely, single parent families; poverty; a lack of love and care; 'shared vulnerability', including difficulties 'mixing' with peers and also on an academic level; victims' 'need for attention'; 'emotional problems'; quiet, shy disposition; victim's previous history of CSA; family history of CSA; and evidence of running away.

It appears that in some cases, as Spiegel (2003: 29) points out, extra-familial abusers who demonstrated a preference for male victims were more likely to 'align with the boy at his developmental level', thus 'fixating' on the 'role of a child'. Intra-familial abusers on the other hand, were more likely to 'elevate the child into the role of an adult'. Of note, is that some of the latter, referred to the part played by parental control in their offending.

Table 7.2 summarises the extent to which Core ITs were present in the men's narratives. The majority of the men (17 out of 23) clearly viewed 'Children as Sexual Objects'. Fifteen referred to a sense of 'Uncontrollability' inherent in their offending. For 11 men, the world is a 'dangerous' place. There was evidence of 'Entitlement' in 4 men's narratives; and 'Nature of Harm' in 3 accounts.

One of the issues which emerged in the narratives of some of the men in this sub-sample was their concern that they continued to present a risk to children; and their need to understand their offending on an individual level in order to desist from offending. Given that 3 of the men who expressed such concern had completed the SOTP, and one man was undertaking it, this warrants further consideration. In working towards desistence, offenders should be enabled to understand their life history and the extent to which it may have influenced their offending behaviour. Indeed, it is crucial that they understand 'why 
they are where they are' (Kear-Colwell and Boer, 2000: 599), so that change might be effected in their behavior (Colton et al. 2009).

In the following Chapter, attention shifts to the 5 men who reported experiencing femaleperpetrated CSA. 


\section{Chapter 8: Research Findings: Experiencing female-perpetrated CSA}

\section{Introduction}

The emphasis here is on the accounts of 5 out of 40 male child sexual abusers who reported at interview that they had experienced female-perpetrated CSA. This is, of course, a very small sample and thus the findings cannot be generalized. However, there is a paucity of research on this population and their experiences of female-perpetrated CSA; therein lies the value of this work and its contribution to cumulative knowledge. As was the case with the 32 men who reported male-perpetrated CSA, the focus, once more, is on the men's experiences of abuse; their perceptions of that abuse; its impact on them; and the extent to which it led to them 'becoming an abuser'. A further 3 men were also abused by females; however, those men were, in addition, abused by males at different points in their childhood. Therefore, their accounts are presented elsewhere, in Chapter 9.

Consistent with the approach adopted throughout this thesis, the profiles of all 5 men are examined at the outset of this chapter. Qualitative data collected during in-depth, individual interviews are then drawn on. In presenting the men's accounts, once more, their names have been changed; and some details omitted in order to preserve anonymity. 


\section{Part 1: Offenders' experiences and perceptions of female-perpetrated CSA}

\section{The 5 offenders}

Table 8.1: Demographic characteristics

\begin{tabular}{|c|c|c|}
\hline$(\mathrm{N}=5)$ & & No. offenders \\
\hline \multirow[t]{4}{*}{ Age: } & $18-30$ & 1 \\
\hline & $31-45$ & 1 \\
\hline & $46-55$ & 1 \\
\hline & $65+$ & 2 \\
\hline \multirow[t]{4}{*}{ Marital status: } & single & 2 \\
\hline & married & 1 \\
\hline & divorced & 1 \\
\hline & widower & 1 \\
\hline \multirow[t]{4}{*}{ Children: } & 0 & 2 \\
\hline & 2 & 1 \\
\hline & 3 & 1 \\
\hline & 4 & 1 \\
\hline \multirow[t]{4}{*}{ Qualifications: } & none & 2 \\
\hline & GCSEs & 1 \\
\hline & higher degree & 1 \\
\hline & other & 1 \\
\hline
\end{tabular}

Offenders ranged in age from 25 years to 76 years at the time of interview. Interestingly, in contrast with Duncan and Williams' (1998) assertion that males abused by females are less likely to marry, only 2 of the 5 men were single. Three of the men had children of their own. Two had no qualifications; one had obtained GCSEs at school; and one man had a higher degree. The remaining man had obtained work-based qualifications. All the men had been employed in unskilled, manual work prior to imprisonment.

Table 8.2 provides some detail of the men's offending behaviour. 
Table 8.2: Offending behaviour

$(\mathrm{N}=5)$

Previous offending:

Current sentence:

$\begin{array}{lr} & \text { No. offe } \\ \text { none } & 2 \\ \text { sexual offences (children) } & 1 \\ \text { theft/fraud } & 2 \\ & \\ <2 \text { years } & 2 \\ 2 \text { years } & 1 \\ >4 \text { years } & 2\end{array}$

Craissati et al. (2002) found that offenders who had been sexually victimised in childhood were more likely to have had previous convictions for sexual offending. However, only 1 of the 5 men in this sub-sample had previous convictions for sexual offending against a child (Colin). He appears to have specialised (Harris et al. 2009) in sexual offending against children over many years. The men were serving prison sentences ranging from less than 2 years to 4.5 years.

In the section which follows, information is presented on the number, age and gender of offenders' victims; and also the duration of abuse. Once more, the aim here is to provide as detailed a picture as possible of the men's offending. 
Table 8.3: Victims + duration of abuse

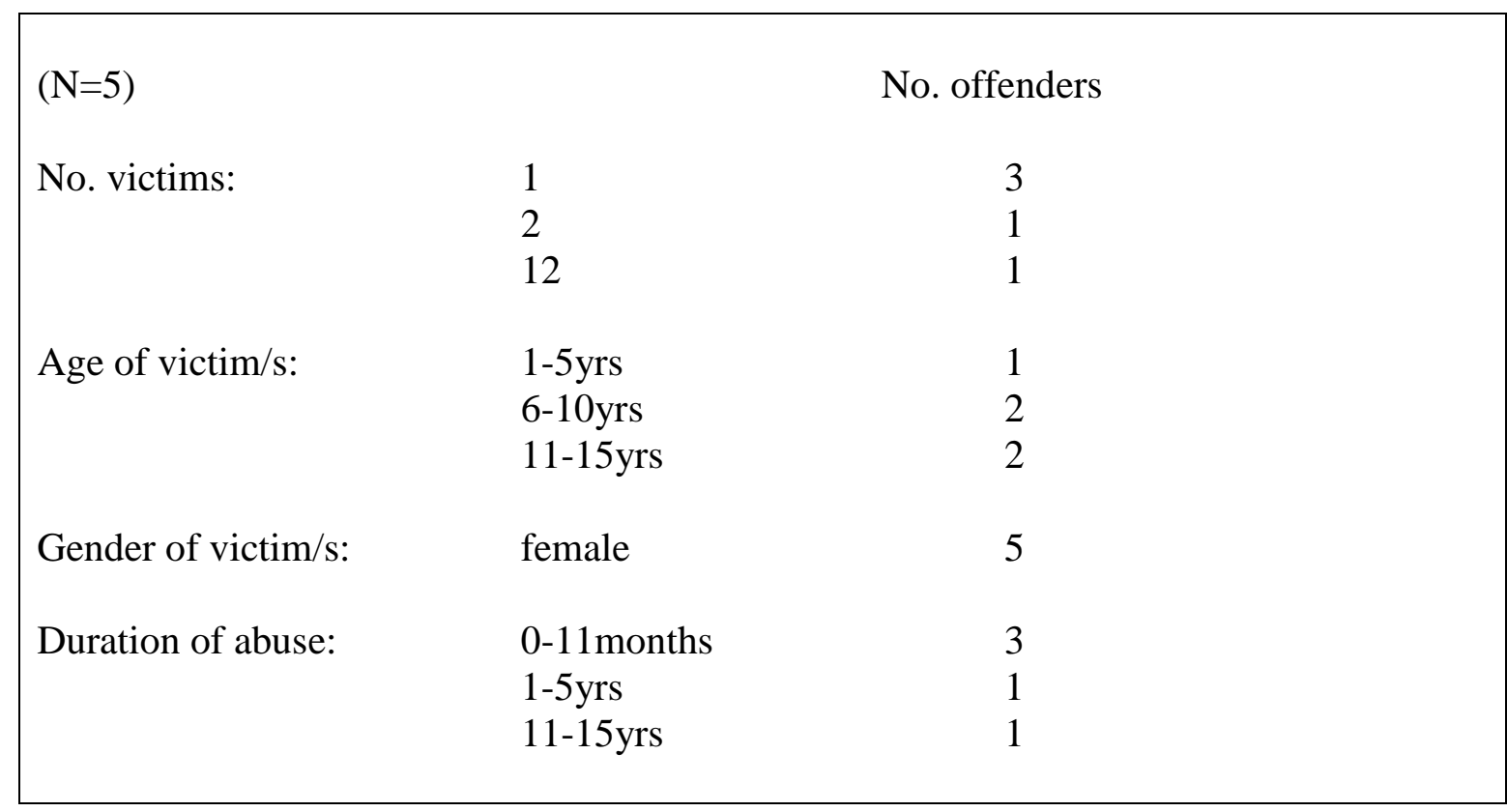

Proeve and Reilly (2007) found that offenders who had experienced sexual abuse by a female in childhood were less likely to abuse multiple victims than offenders who had experienced male-perpetrated abuse. The majority of men (4) in this sub-sample had abused either 1 or 2 victims. One man, however, - who had been abused by his sister in childhood - had offended against 12 victims. Those victims ranged in age from 4-15 years.

The subject of victims' gender raises some interesting issues and these are worth considering here. Briggs (1995: xii), posits that: 'the gender of the initial abuser determines the gender of later victims'. In the vast majority of cases, Briggs found that: boys abused by males subsequently abused boys; boys abused by females, abused female victims; and those who had been abused by males and females abused victims of both sexes. Moreover, Craissati et al. (2002) note, that offenders who had been sexually abused in childhood by males were also more likely to abuse male victims. While some other researchers (Romano and DeLuca 1997) have found similarities between the gender of those who abused offenders in childhood and their later victims, offenders in Proeve and Reilly's (2007) sample were more likely to have victimised both males and females. 
It is interesting to note that all 5 offenders in this sub-sample abused female victims. This is consistent with Briggs’ (1995) findings.

Four men had offended in their own home. Two of those men also offended in other locations, including friends' or relatives' homes; their cars; and victims' homes. Three of the men abused their victims over a period of less than 1 year. Four men perpetrated abuse over a period of 1 to 5 years; and one man abused 12 victims over a 12 year period, moving on from one victim to another in that time.

Table 8.4 provides further detail of the offences committed by the men; and their relationship to their victims.

Table 8.4: Offence/s \& offenders' relationship to victim/s

\begin{tabular}{|l|l|l|l|}
\hline $\begin{array}{l}\text { Offenders: } \\
(\mathrm{N}=5)\end{array}$ & Offence/s: & Relationship to victim/s: & Relationship (contd.): \\
\hline Andrew & Indecent assault & $\begin{array}{l}\text { Stepdaughters (15 \& 12 } \\
\text { years of age) }\end{array}$ & Intra-familial \\
\hline Bill & Indecent assault & $\begin{array}{l}\text { Girlfriend's daughter (6 } \\
\text { years) }\end{array}$ & Extra-familial \\
\hline Colin & Indecent assault & $\begin{array}{l}\text { Known female - neighbour } \\
\text { (10 years) }\end{array}$ & Extra-familial \\
\hline Eric & Gross indecency & $\begin{array}{l}\text { Stepson's partner's } \\
\text { daughter (age not } \\
\text { specified) }\end{array}$ & Intra-familial \\
\hline Gareth & $\begin{array}{l}\text { Indecent assault \& } \\
\text { Gross indecency }\end{array}$ & $\begin{array}{l}\text { Nieces; cousins; } \\
\text { neighbour; \& friend of } \\
\text { family (x12 female } \\
\text { victims; 4-15 years) }\end{array}$ & Intra- and extra-familial \\
\hline
\end{tabular}

Some evidence indicates that child sexual abusers who were themselves abused in childhood are more likely to abuse extra-familial victims (Salter et al. 2003). Other researchers, however, (Proeve and Reilly 2007) have found that their offending is more likely to span relationship boundaries; and to be perpetrated against both intra- and extrafamilial victims. As can be seen from Table 8.4, 2 of the men perpetrated abuse on an intra-familial level; 2 men abused extra-familial victims only; and in one case, where 
there were 12 victims, the majority (10) of those were intra-familial; and 2 were extrafamilial.

Two men - Colin and Gareth - appear to fall into the category of 'fixated' offenders: (Groth and Birnbaum 1978: 176). Colin reported a sexual preference for female victims, and of having been sexually attracted to children: 'all my life - girl children'. Moreover, Gareth had perpetrated abuse from the age of 12. One of the men in this group - Bill was undertaking the SOTP at the time of interview.

Having explored the men's characteristics and offending behaviour, attention now turns to their reported childhood sexual victimisation: first, their relationship to their abuser. 
Table 8.5: Offenders' childhood sexual victimisation relationship to perpetrator

$(\mathrm{N}=5)$

Perpetrators' relationship to offender:

Offender:

Mother (I/F)

Aunt (I/F)

Sister (I/F)

Grandmother (I/F)

Teacher $(\mathrm{E} / \mathrm{F})$

BILL

COLIN

GARETH

ANDREW

ERIC

Key: I/F - intra-familial; E/F - extra-familial.

As can be seen from Table 8.5, the women who abused offenders in this sub-sample were either related to, or well-known to their victim. Four of the 5 men experienced intrafamilial abuse. This involved a mother; grandmother; aunt; and sister. The remaining man was abused on an extra-familial level by someone in a position of trust: a teacher. This is in contrast to the findings of other researchers such as Craissati et al. (2002) and Proeve and Reilly (2007: 257), who reported that most of the child sexual offenders in their research who had experienced sexual victimisation by females in childhood, had been abused by acquaintances or strangers: these were usually 'older female acquaintances'.

Little attention has been paid to examining the abuse perpetrated by females ‘independently’ of males (Gannon and Rose 2008: 443). Some research indicates that lone perpetrators are more likely to abuse males (Bourke et al. 2014; Muskens et al. 2011; Vandiver 2006). All of the men in this sub-sample reported that the abuse they experienced was perpetrated by solo offenders, as was the case in Deering and Mellor's (2011) research.

Child sexual abuse perpetrated by women ranges from: genital fondling; to oral sex and sexual penetration (Vandiver and Walker 2002; Vandiver and Kercher 2004), including digital penetration and penetration with objects (Elliott 1993). The abuse experienced by the 5 men in this sub-sample included exposure; fondling; masturbation; simulated sex; 
and full sexual intercourse. With the exception of one man, all of the men in this sample reported having first been abused between the ages of 9 and 13 years. One man was aged 13 at the onset of abuse; 3 men were 12 years old; one was 11 years of age; one 10 years; and one 9 years old. The remaining man, however, had been abused by his mother while a very young child, ‘from as early' as he could remember.

Two of the 5 men in this group - Colin and Gareth - reported experiencing physical abuse as well as sexual abuse in childhood. This was not perpetrated by their abuser, but by a father in one case; and other male pupils at school in another.

Having provided some detail of offenders' backgrounds; their offending behaviour; and the CSA they experienced, attention now shifts to the men's accounts of that abuse; their perceptions of it; its impact on them; and the extent to which it played a part in their subsequent sexual offending. These accounts are presented verbatim and are ordered according to the 3 themes below, which emerged from analysis of the interview data:

- Offenders' experiences of female-perpetrated child sexual abuse

- Offenders' perceptions of female-perpetrated child sexual abuse

- Becoming an abuser

The men's experiences and perceptions of their childhood sexual victimisation are presented in Part 1 of this Chapter. Their account of 'becoming an abuser' is contained in Part 2. Once more, distinctions are made between groups: namely, between those reporting intra-familial and extra-familial abuse in childhood - an approach adopted in an attempt to tease out difference where that may exist.

\section{Experiencing intra-familial CSA}

Four of the 5 men who had been abused solely by females, had experienced intra-familial CSA. Andrew had been abused by his grandmother; Bill by his mother; Colin by an aunt; 
and Gareth by his sister. The men's accounts of that abuse follow. In each case, their accounts are preceded by some contextual, and individual, detail on the men.

Andrew was 51 at the time of interview and divorced. He had been convicted of indecent assault against his 2 step-daughters who were aged 15 and 12 years respectively. The offences were committed at the family home. Andrew reported having been abused by his grandmother from the age of 10 .

Most FSOs range in age between 16 and 36 years (Vandiver and Kercher 2004), with a mean age of 26 years (Faller 1987). Research activity has focused on maternal sexual abuse (Banning 1989; Lawson 1993) with less attention paid to abuse perpetrated by other older females, in particular, grandmothers. This is perhaps unsurprising given that stereotypical images of grandparents present them as 'kindly and solicitous' individuals (Margolin 1992: 735). Margolin (1992) draws attention to the gendered nature of grandparent abuse, with most perpetrators being male and most victims female. Given this, Andrew's account of his abuse by an older woman - his father's mother - provides a useful insight into a relatively unexplored area. He reported having been abused by his grandmother from the age of 10 . By the time he reached 13 or 14 they were engaging in full sexual intercourse. Andrew had been introduced to sexual activity characterised by the use of implements and pain at a young age; and, in retrospect, he reflected on how 'awful' the weekends were:

'My nan used to give me all sorts of things to stick inside her - bottles, broom handles. I wasn't aroused at first but at 13 or 14 she realised I could get an erection. The weekends were awful. She'd want it all weekend. She'd walk around the house naked. She used to want oral sex and through the vagina and back passage'.

Bill was 34 and single when interviewed. He had been convicted of indecent assault against his girlfriend's 6 year old daughter. The offences were committed at his victim's home over a period of 4 months. He reported having been sexually abused by his mother as a child 'from as early' as he could remember. 
Mother-son sexual abuse is a particularly hidden form of offending due, in part, to the challenges faced in defining such abuse (Lawson 1993); and societal rejection that females may be abusers (Spiegel 2003). Bill's account sheds some light on this form of abuse. As was the case with Andrew, abuse was a regular occurrence for Bill and began when he was very young:

'It was by my mum - she masturbated me basically from as early as I can remember'.

Colin was the oldest man in this sub-sample. He was 76 at the time of interview and a widower. Colin was the only man in this group to have previous convictions for sexual offending. His sexual offending history spanned many years. He had 3 previous convictions for indecent assault for which he had received 2 prison sentences. The first offence had been committed 30 years prior to interview; and the second, 20 years before interview. The remainder were more recent offences, having been committed 5 and 2 years respectively before interview. His previous female victims were aged 10, 14 and 15 years; all were friends of the family or neighbours. All of those offences had been committed in Colin's own home. Most recently, Colin had been convicted of indecent assault against a neighbour's child, who was 10 years of age. In that case, the abuse was perpetrated in his car. Colin had been abused by his aunt as a child.

While the majority of those who engage in SHB are male (Hackett et al. 2013), increasing attention is being drawn to adolescent females who also have the capacity to sexually abuse (Kubik et al. 2002). Females are more likely to have several victims, who are male and family members; and to engage in multiple episodes of offending against victims (Finkelhor et al. 2009). Much abuse perpetrated by adolescents occurs in the context of non-parental caregiving, for example, as part of the babysitting role (Margolin and Craft 1990). There has, however, been a tendency to disregard the 'seriousness' of sexual offending by children and young people, which has often been dismissed as sexual experimentation or play (Johnson 1988: 219). Colin reported having been abused 'on a regular basis' as a child, by his young aunt. The abuse persisted for a number of years 
and was perpetrated in his home while his aunt was babysitting, and 'also afterwards as a youth':

'I was offended against myself as a child. I had a young auntie - I was 9 and she was 14, 15 and we were boys and girls together. It was a large family of cousins and so forth and she often used to watch us younger ones when mum and dad went out. And then those activities ceased and I started work at $14 \ldots$...She started again before I joined the Army and then I never saw her again after that...It was groping her and touching her breasts and things like that...She'd masturbate me on a regular basis.'

Gareth was the youngest man in this sub-sample. He was 25 years old and single when interviewed. He had no previous convictions. He had been convicted of indecent assault and gross indecency against 12 female victims aged between 4 and 15 years. All were family members - nieces and cousins - with the exception of 2 victims, who were family friends. The offences were committed over a 12 year period at Gareth's home and also the victims’ homes. Gareth reported having been sexually abused by his older sister from the age of 11 .

Sibling abuse is hidden and under-reported (Stathopoulos 2012). Yet, research indicates that this form of abuse is likely to be more prevalent than other forms of intra-familial abuse (ibid.). Of interest, is that sibling abuse is most commonly perpetrated by brothers against sisters (Carlson et al. 2006). At first, Gareth was extremely reluctant to talk about his childhood; and initial attempts to establish whether he had been sexually victimized as a child proved unsuccessful. Some time was therefore spent reassuring Gareth and this was discussed in some detail in the Methodology. Once reassured, Gareth began to talk about his childhood. At first, he reflected on his social isolation from his peers; his dislike of school; and of people:

'How can you possibly like a place where you ended up getting bullied, you ended up having the mickey taken out of you. When I was in my first senior school we had a big skip in the playground and I spent more time in the skip than I did in the school. There was bunch of kids who used to grab me and chuck me in there. It wasn't one of those open topped skips that you see lying around, it was one of those big square ones with lockable doors so they weren't as easy to get out of. All I could do was bang on the walls until somebody came and heard me. I 
don't like people, people as a whole. The human race isn't worth the planet that it's on.'

Gareth described how he first started abusing family members after being abused himself by his older sister, who was 16 years of age at the time:

'I started abusing when I was 12. I was 11 [when my sister abused me] and it was a couple of weeks after she stopped touching me that I touched my niece'.

Evidence suggests that children who engage in SHB are more likely to abuse within their own family, as Gareth did at first; this may be due to their limited access to other children. They are also more likely to have a history of CSA in their backgrounds (Johnson 1988). Of note in Gareth's case, is the prolific nature of his offending, which amounted to abuse against 12 victims before he was apprehended and imprisoned.

\section{Experiencing extra-familial CSA}

Eric was 68 and married when interviewed. He had been convicted of gross indecency against his stepson's partner's child. The abuse was perpetrated over an 18 month period at his own home; his victim's home; and in his car. Eric was the only one of the 5 men in this group who had experienced abuse on an extra-familial level in childhood. He had been sexually abused by an adult in a position of trust - his female teacher - at the age of 12. He was, however, keen to minimize the abuse and there is some evidence of invalidation (Spiegel 2003) in his narrative:

'I was interfered with - but it never affected me in my mind - by a teacher, a woman teacher. But as far as I was concerned when school was finished, it was finished. At the time I think I was about 12'.

\section{Categorizing the abuse experienced}

Notwithstanding the limitations of this research, in particular, the small sample size, these men's accounts provide some insight into childhoods which were characterised by sexual activity from a young age. The abuse they experienced ranged from: exposure; to fondling; masturbation; simulated sex; and full sexual intercourse. Table 8.6 provides 
information on the nature of that abuse; the age of onset and duration; and attempts to interpret the men's abusive experience with reference to Lawson's (1993) categories of abuse referred to in Chapter 2: subtle; seductive; perversive; overt; and sadistic.

Table 8.6: Categorizing abuse

\begin{tabular}{|l|l|l|l|l|l|}
\hline $\begin{array}{l}\text { Offender: } \\
(\mathrm{N}=5)\end{array}$ & Abused by: & $\begin{array}{l}\text { Nature of } \\
\text { abuse: }\end{array}$ & $\begin{array}{l}\text { Form of } \\
\text { abuse: }\end{array}$ & $\begin{array}{l}\text { Age at onset } \\
\text { of abuse: }\end{array}$ & $\begin{array}{l}\text { Duration of } \\
\text { abuse: }\end{array}$ \\
\hline Andrew & Grandmother & FSI & Overt & 10 years & 7 years \\
\hline Bill & Mother & M & Overt & $\begin{array}{l}\text { 'from as } \\
\text { early as I } \\
\text { can } \\
\text { remember' }\end{array}$ & $\begin{array}{l}\text { 'many } \\
\text { years' -- } \\
\text { until age of } \\
15\end{array}$ \\
\hline Colin & Young aunt & $\mathrm{M}$ & Overt & 9 years & 5 years + \\
\hline Eric & Female teacher & F & Overt & 12 years & $\begin{array}{l}\text { 'a few } \\
\text { months' }\end{array}$ \\
\hline Gareth & Sister & $\mathrm{M}$ & Overt & 11 years & 1 year \\
\hline
\end{tabular}

[Key: FSI = full sexual intercourse; $M=$ masturbation; $F$ = fondling]

All 5 men appear to have experienced overt abuse: that is, abuse characterised by 'overtly sexualised contact' (Lawson 1993: 266). This form of abuse ranges from intercourse; to fondling of the child's genitals; coercing the child to fondle the female's breasts or genitals; cunnilingus; fellatio; digital penetration (or penetration with objects) of the child's vagina or anus; and sexualized touching and kissing (Saradjian 1996).

As already noted in Chapter 2, Denov (2003), more recently classified female-perpetrated abuse - based on its severity - as follows:

Severe sexual abuse - for example, sexual intercourse; penetration (anal or vaginal) with objects; or digital penetration; fellatio; and cunnilingus

Moderate sexual abuse - for example, genital fondling (without penetration); and simulated intercourse 
Mild sexual abuse - for example, sexualized kissing

It appears that the 5 men in this sub-sample experienced what Denov (2003) refers to as severe or moderate sexual abuse. One of the men experienced the former: full sexual intercourse; and 4 the latter: masturbation and fondling. Three of the 5 men experienced abuse over a protracted period of time. One man had been abused by his grandmother over a 7 year period from the age of 10. Another man - who had been abused by his mother - experienced abuse which began when he was a very young child and spanned 'many years', ending when he was 15. A third man had been abused by a young aunt for over 5 years. Yet another, had been abused by his sister for one year; and the remaining man was abused by his teacher for 'a few months'.

\section{Disclosing abuse}

Disclosing abuse is a complex, painful process which is unique to each victim (Durham 2003). Victims of CSA frequently experience isolation; are forced into remaining silent; and often tell no-one about the abuse (ibid.). This situation is compounded where abuse is perpetrated by females. Having heard the men's accounts of the abuse they experienced, attention now turns to the extent to which they disclosed that abuse; the response they received; and why they chose not to tell.

Abuse of males is under-reported (Finkelhor 1984), with the reluctance to disclose attributed to the prevalence of societal perceptions of males as able to protect themselves from harm (ibid.). Where the perpetrator is female, the taboo associated with FSO (Denov 2004); and the emotional distress which results from the abuse (Faller 1987), prevents many victims from disclosing. Other factors also play a part in victims' inability or reluctance to disclose female-perpetrated abuse, including societal norms which endorse and even glorify older woman/younger boy relationships and fantasies (Hunter 1990) and thus, inhibit disclosure. 
It is unsurprising that only one man in this sub-sample, Andrew, disclosed the abuse he experienced in childhood. Denov's (2003) assertion, that the level of harm experienced by victims of female-perpetrated abuse is often minimized, and victims' experiences trivialized, is illustrated by Andrew's attempts to tell of the abuse perpetrated by his grandmother. His disclosure was met with inappropriate - and inadequate - responses from his parents:

'At 10 my grandmother started molesting me. I told my mother but she turned a blind eye and said, nan's lonely, don't be silly, nan loves you very much. The abuse continued until I started work and then I realised it was wrong after talking to workmates about sex. My grandmother was in her 40s and had just lost her husband. I mentioned it to my father who was very rarely around and he just smiled. Both parents knew and ignored it'.

Andrew's attempts to understand his grandmother's abusive behaviour appear to have been influenced by his parents' response, and their interpretation of her behaviour as resulting from her loneliness. In this case, Andrew perhaps served as a substitute for a lost husband. It was not until some years after the abuse began and Andrew started work that he realised, during the course of conversations with 'workmates', what was happening to him was wrong.

Bill made clear that, prior to interview, he had not previously disclosed his abuse by his mother. That decision appears to have been based on fear of the consequences:

'I haven't said anything to anyone about it before, and I wouldn't want to take it any further. Do you know what I mean? I wouldn't want to take it to court or anything like that. I don't see the point in that - it would be too destructive for everybody.'

Bill's comments appear to confirm Finkelhor's (1986) proposition that the fear of reporting abuse and its consequence for the victim's family - and loyalty towards them augments the secrecy (Summit 1983) surrounding abuse. Family loyalty clearly featured for Bill. He was the only man in this group undertaking the SOTP at the time of interview, and he explained that he had not disclosed the identity of his abuser to Programme staff: 
'I said a family member abused me as a kid but I didn't say which family member it was. I probably would have done if they had asked but I didn't particularly want to go into details about that'.

Evidence suggests that victims of female-perpetrated abuse encounter a more profound sense of isolation (Deering and Mellor 2011) thought to result from the 'silence' surrounding this form of abuse. Those abused by siblings are less likely to disclose than other victims (Stathopoulos 2012). It is not surprising, therefore, that Gareth - who had been abused by his sister - did not tell. Sibling sexual abuse may emanate from family dysfunction (ibid.); and poor relationships with parents characterized by a lack of affection and emotional distance (Hatch 2005). Although Gareth was part of a large family, non-disclosure of the abuse perpetrated by his sister appears to have been due, in part, to his isolation (Bascelli et al. 2004; Jensen et al. 2005; Sorsoli et al. 2008; Tutty et al. 2009). When asked at interview whether he had ever told anyone of the abuse he had experienced, Gareth replied:

'Who could I tell? I weren’t close enough to anyone to tell them'.

One of the 5 men in this sub-sample, Eric, reported being abused by someone in a position of trust, or professional perpetrator (Moulden et al. 2007): a female teacher. Children abused by professional perpetrators, particularly educators, are less likely to disclose than other victims (Roberts and Vanstone 2014). Given this, and the additional element of female as abuser, it is unsurprising that Eric did not disclose the abuse perpetrated by his teacher.

Having explored offenders' experiences of female-perpetrated CSA, and the extent to which they disclosed that abuse, attention now shifts to their perceptions of CSA and its impact on them. 


\section{Offenders' perceptions of female-perpetrated CSA}

\section{Introduction}

Saradjian and Hanks (1996) argue that because women are not expected to perpetrate sexual violence against children, victims experience more prolonged abuse; greater stigmatization; an increased sense of difference; and a greater sense of betrayal. Consequently, victims are unable to engage in healthy relationships in adulthood, and some may become abusers themselves. The limited body of research on impact and perceptions of female-perpetrated abuse is, however, characterized by conflicting evidence. Moreover, missing, are the views of child sexual abusers on their own childhood sexual victimization by females. Those views feature here, with the aim of addressing that gap in knowledge.

While the use of force or violence was often reported by participants in Deering and Mellor's (2011) study, none of the men who participated in this research reported the use of force in their abuse. The section which follows focuses on offenders' perceptions of the abuse they experienced. Of interest, is that their narratives are permeated by positive interpretations of their victimisation. 
Table 8.7: Offenders' perceptions of their childhood sexual victimisation + details of their subsequent offending

\begin{tabular}{|l|l|l|l|l|l|}
\hline & Abused by: & $\begin{array}{l}\text { Nature of } \\
\text { abuse: }\end{array}$ & $\begin{array}{l}\text { Viewed } \\
\text { abuse: }\end{array}$ & Offence/s: & Victim/s: \\
\hline Andrew & $\begin{array}{l}\text { Grandmother - } \\
\text { from 10 years } \\
\text { of age }\end{array}$ & FSI & Positively & $\begin{array}{l}\text { Indecent } \\
\text { assault }\end{array}$ & $\begin{array}{l}\text { Stepdaughters } \\
\text { (15 \& 12 } \\
\text { years of age) }\end{array}$ \\
\hline Bill & $\begin{array}{l}\text { Mother - } \\
\text { from 'early as } \\
\text { can remember }\end{array}$ & M & Positively & $\begin{array}{l}\text { Indecent } \\
\text { assault }\end{array}$ & $\begin{array}{l}\text { Partner's } \\
\text { daughter (6 } \\
\text { years) }\end{array}$ \\
\hline Colin & $\begin{array}{l}\text { Young aunt - } \\
\text { from 9 years } \\
\text { of age }\end{array}$ & M & $\begin{array}{l}\text { Positively } \\
\text { - from 12 } \\
\text { years of age }\end{array}$ & $\begin{array}{l}\text { Indecent } \\
\text { assault }\end{array}$ & $\begin{array}{l}\text { Known } \\
\text { female (10 } \\
\text { years) }\end{array}$ \\
\hline Eric & $\begin{array}{l}\text { Semale teacher } \\
\text { fister - from years of age }\end{array}$ & F & Positively & $\begin{array}{l}\text { Gross } \\
\text { indecency }\end{array}$ & $\begin{array}{l}\text { Stepson's } \\
\text { partner's } \\
\text { daughter (age } \\
\text { unknown) }\end{array}$ \\
\hline Gareth & Positively & $\begin{array}{l}\text { Indecent } \\
\text { assault \& } \\
\text { gross } \\
\text { indecency }\end{array}$ & $\begin{array}{l}\text { Nieces; } \\
\text { cousins; \& } \\
\text { friends of } \\
\text { family (x12 } \\
\text { female } \\
\text { victims 4-15 } \\
\text { years) }\end{array}$ \\
\hline
\end{tabular}

Key: FSI = full sexual intercourse; $M=$ masturbation; $F=$ fondling

Andrew had been abused by his grandmother from the age of 10, and talked of eventually 'enjoying' the sexual activity:

'In the end I was enjoying it. I was going there while I was going out with my wife [when she was my girlfriend]. We tried having full sex at 12 or 13 and had full sex at 13 or 14 '.

As noted earlier, Andrew had tried to tell both parents of the abuse but it was 'ignored'. Thus, because he was not reprimanded, it appears that Andrew normalised the abuse:

'thought it was OK because they didn't tell me off. I took it as being natural really'.

He described 'falling in love' with his grandmother, who gave him 'everything' he wanted in a material sense. Interestingly, his narrative sheds some light on the extent to 
which retrospectively, he recognised his own vulnerability as a child; the extent to which his grandmother exploited that; and the sense in which he felt 'different' to his siblings:

'Looking at it now as an adult, I suppose I fell in love with her. I was nan's pet. Everything I wanted she gave me but wouldn't give to my brothers. She didn't abuse anyone else in the family. I have difficulty reading and writing and wasn't as clever as the others and I suppose she took advantage of that. I was always slow and I think she used that. When she died she left me all the contents of her will. Dad said, nan loved you and she's paying you back for being a good boy'.

Andrew clearly appreciated being 'nan's pet' and the rewards he received from her. However, by the time he was 17 , he had a strong sense that the sexual activity was 'wrong' and was fearful that it would be discovered. That led to him ending 'the abuse':

'I stopped it myself because I realised it was wrong. She was the pillar of the family but it had to stop. I told nan and she was quite upset. I still saw nan after I finished the abuse. She tried to get me into bed but I wouldn't. I had a girlfriend and by that time (aged 17) I realised it was wrong and I was frightened of people finding out'.

Hudson (2003) notes that the sexual offenders she interviewed, in her research on their treatment and management, found talking about their offences in individual interviews therapeutic. Some of the men in this sample appeared to share that view. Here, Andrew (who had abused his stepdaughters) reflects more negatively on the impact of his victimisation. He articulates the guilt he felt at having been abused; and the 'torment', 'anger and frustration' he experienced as a result. He is clear that talking about his own victimisation, and understanding it, is helpful in preventive terms:

'Since I've been in here my past is out. It's been a torment and I want to get it out of my system and off my conscience. [Talking] gets rid of the anger and frustration I've bottled up'.

While Andrew believed that he had fallen in love with his grandmother and that the abuse was 'natural', later in the interview, his recollection of his grandmother became more negative and he referred to her as:

'a dirty, disgusting bitch'. 
Denial of harm, and a failure to view the abuse they had experienced negatively permeates the accounts of several of the men in Etherington's (1997) study. Indeed, some of the men in that study were protective of their mothers and minimised the abuse perpetrated against them. One man perceived his abuse as a 'demonstration' of his mother's love for him and there are parallels there with Bill's account of his abuse. Bill talked of becoming accustomed to - and like Andrew - enjoying the abuse perpetrated by his mother. He perceived the abuse to be a non-violent act, loving, non-threatening, and seemingly normal:

'It didn’t happen all the time, and it wasn't done in a nasty sort of violent way, but sort of loving, quote, unquote loving - a non-threatening sort of a way, so it just seemed normal. I got used to it, enjoyed it.'

Most CSA is not perpetrated 'under conditions of danger, threat, and violence' (Finkelhor 1987: 352). It is unsurprising therefore, that some victims only recognise they have been abused some time later, when they develop an understanding of 'appropriate sexual conduct' (ibid.). This is of relevance to both Bill and Andrew. While at first, Bill perceived the abuse as a normal part of life, as he grew older he, like Andrew, realised that this sort of behaviour was not normal:

'Once I got up to about 13 or round that age - early teens - I realised this wasn't normal and kind of shied away from it then, and lost a lot of respect for my mum. My parents had divorced, separated when I was 11. [They] finally divorced when I was 13, so that was happening around that time as well. My dad was always working so he wasn't around an awful lot. He was basically a decent sort of a guy, a bit short tempered at times but he wasn't violent or abusive'.

Although he viewed his abuse as a 'loving' act, Bill described his relationship with his mother as 'quite cold'. Like Andrew, having perceived the abuse positively, during the course of the interview he reflected more negatively on his mother.

Victims are often unaware that siblings have also been abused: sometimes this is disclosed in adulthood, but often it is not (Briggs 1995). Bill was unsure whether his younger brother had also been abused by his mother: 
'I wasn't especially close to her growing up. I suppose deep down, I always suspected that there was something weird but my young brother tended to be closer to her, so she tended to have a lot more affection and cuddles and stuff with him. Whether or not she was doing anything with him, I don't know'.

The 3 remaining men also had positive perceptions of the abuse they experienced in childhood: from a young aunt; a female teacher; and a sister.

Colin's perception of the abuse he experienced was overwhelmingly positive. He had been abused by his young aunt and talked of 'delighting' in the abuse:

'She used to show me her body and ask me to do various naughty things. I am not going to say I didn't delight in it because as a boy, of course I did, naturally I did'.

As already noted, while sibling abuse is the most prevalent form of intra-familial abuse, there is scant information as to its impact on victims (Stathopoulos 2012). Of concern, is that sibling abuse has consistently been: 'ignored, minimized or denied by parents, professionals and authorities as benign childhood sexual experimentation' (ibid.: 16).

In Gareth's case, following his own abuse, he subsequently perpetrated abuse against multiple victims over many years. His childhood was highly sexualized; and his account of the sibling abuse he experienced in childhood, provides some insight into that. Gareth had an extremely positive view of the abuse he experienced. He believed that the abuse perpetrated by his sister had been 'an interesting game' which he had been taught. It led to the pursuit of sexual gratification through the abuse of multiple victims:

'I started abusing when I was 12. I didn't even know it was wrong then. As far as I was concerned it was like a game. It was an interesting game and I had been taught it. And I just carried on doing it [to family members]. My sister awakened me to the actual pleasures of sex and that is when I got into full gratification, rather than just a game. That is when I got into it for actual gratification'.

Cases involving adult females and adolescent boys may be unreported because of the tendency, on the part of society and the victim, to view them as an initiation (Hines and Finkelhor 2007) into the realms of sexual activity. Thus, male victims are less likely to perceive the 'relationship' as abuse or to acknowledge that there may be any resulting negative impact (Gannon and Rose 2008). This is perhaps of relevance in Eric's case. He 
had been abused by a female teacher at the age of 12 and, like the other 4 men, had positive perceptions of the abuse:

'I'm not being funny but I enjoyed it to be honest. It was only a few times; a few months - that was it'.

\section{Summary: Part 1}

In Part 1 of this Chapter, the focus has been on 5 men's experiences and perceptions of female-perpetrated CSA. Three of the 5 men in this sub-sample were, or had been married; and 3 had children of their own. Only one man (Colin) had a previous conviction for sexual offending against children. In contrast with the 32 men abused by males, the majority (4 out of 5 ) of men in this sub-sample were abused on an intrafamilial level. One had experienced maternal abuse; another had been abused by his grandmother; and 2 men were abused by other young people: a sister and a young aunt. The remaining man had been abused by a teacher.

Research evidence suggests that certain characteristics associated with the experience of CSA may result in an increased risk of sexual offending (Proeve and Reilly 2007). These characteristics include being abused by more than one perpetrator; frequent abuse over lengthy periods of time (Briggs and Hawkins 1996; Freeman-Longo 1986); and femaleperpetrated abuse. All of the men in this sub-sample reported having been abused by females, in some cases, over several years. Of note, is that the men's accounts of their sexual victimisation differed in terms of the role played by the perpetrator in their life; and the nature of the abuse they experienced. There appears to be some consistency, however, in their perceptions of that abuse, which were overwhelmingly positive.

Understanding of female-perpetrated CSA is impeded by myth: namely, that females do not sexually abuse; they only abuse if coerced by a male; that female-perpetrated child sexual abuse is 'harmless', gentle, loving, or misguided 'motherly' love; and that women only sexually abuse adolescents (Longdon 1993).The accounts of offenders in this subsample dispel some of these myths. All had been abused by lone females, none of whom 
had been coerced by a male. Moreover, some of the men were very young at the onset of abuse. With the exception of one man (Bill), all the offenders in this sub-sample reported having first been abused between the ages of 9 and 13 years. Andrew's abuse included full sexual intercourse. Bill, Colin and Gareth had experienced masturbation; and Eric fondling.

There are a number of factors which might have contributed to these men's vulnerability to CSA. Andrew appears to have had some learning difficulties; Bill's father was often absent and his relationship with his mother (who abused him) was 'quite cold'. Colin and Gareth experienced physical violence in childhood. Moreover, Gareth was socially isolated and found it difficult to engage in relationships with his peers.

The majority (4 out of 5) of the men did not disclose the abuse they experienced. For the one man who did disclose (Andrew), the parental response was inappropriate; the abuse persisted and increased in severity. Bill chose not to disclose the maternal abuse he experienced: family loyalty featured here. Gareth's isolation appears to have played a part in his non-disclosure of sibling abuse. Perhaps the status of Eric's abuser (a teacher) inhibited his telling; and it is possible that the sexual abuse perpetrated by Colin's young aunt was masked - and telling inhibited - by her babysitting role, given that she had been charged by adults with the care of children.

In both a popular and professional sense, sexual abuse perpetrated by women is deemed to be relatively 'harmless' when compared with sexual abuse by males. Yet, some victims' perceptions indicate otherwise: namely, that abuse by females is more harmful, resulting in a 'deeper sense of betrayal' (Denov 2004: 1153). Of interest, is that all 5 men in this sub-sample viewed the abuse they had experienced positively. Indeed, each of the men used positive descriptors when referring to their abuse, including phrases such as: 'enjoying' the abuse; seeing it as 'natural', 'normal' and a 'loving' act; 'falling in love' with their abuser; 'delighting' in the abuse; seeing it as a game; and as extremely pleasurable. None of the men referred to the abuse as having a negative impact. However, both Andrew and Bill did reflect more negatively on the abuse they experienced as the 
interview progressed, with Andrew, for example, referring to the 'torment', 'anger and frustration' he experienced in later years.

Children who keep their sexually abusive experiences secret experience greater levels of distress than those who disclose and receive appropriate support (Browne and Finkelhor 1986). Moreover, negative responses to disclosure often have deleterious consequences (ibid.). It is crucial that victims' experiences of female-perpetrated CSA are acknowledged through supportive (Denov 2004), and appropriate responses; and imperative that further understanding of the impact of that abuse is gained across victim groups, particularly in relation to child sexual offenders. Hearing their accounts of their abusive experiences, and their perceptions of those, has the capacity to enable more informed and effective treatment and intervention.

Having explored offenders' accounts of the female-perpetrated CSA they experienced; their perceptions of that; and its impact on them, attention now shifts to the extent to which, in their view, the abuse played a part in their subsequent offending. 


\section{Part 2: Becoming an abuser}

\section{Introduction}

Here, offenders' accounts of becoming an abuser are explored. As was the case with the 32 men who reported male-perpetrated CSA, first, an attempt is made to determine to what extent the 5 men in this sub-sample identified links between their own childhood sexual victimisation and their later offending. Then, attention shifts to exploring the presence of Core ITs in their narratives, as a means of understanding the belief systems which supported the perpetration of abuse.

During interview, all the men were asked the following questions in order to determine their understanding of the origins of their sexual offending. The questions were designed to incorporate any abusive or negative experiences in childhood:

- To what extent do you see a link between your own family experiences as a child and your offences against children?

- To what extent do you see a link between your relationship with childhood friends, inside and outside school, and your offences against children?

- To what extent do you see a link between your relationship with any significant adult(s) outside your immediate childhood family and your offences against children?

\section{Making the link}

Three of the 5 men (Andrew, Bill and Gareth) identified links between their family experiences and their later offending, in particular, in relation to the abuse they experienced. Andrew reported a 'very strong' link; and Bill and Gareth a 'fairly strong' link. However, none were able to provide further detail on how those links manifested 
themselves when first asked. Later, however, during the course of the interview, those links became clear; those data are included later in this section of this thesis. Only one man, Bill, reported a 'very strong' link between childhood friends and his offending. None of the men made links between their relationship with significant adults in childhood and their later offending.

In an attempt to determine awareness of when their sexual attraction to children emerged - and whether perhaps offenders could be classified as either fixated or regressed - the men were also asked:

- How old were you when you first became aware that you were sexually attracted to children?

Three of the 5 men (Bill, Colin and Gareth) identified the age at which they became aware that they were sexually attracted to children. For Bill, this awareness emerged at the age of 31; Gareth, reported that he had been sexually attracted to children from the age of 16; and Colin: 'all my life’.

In order to determine their understanding of the origins of their sexual attraction to children, offenders were also asked:

- How do you think you came to be sexually attracted to children?

And as a means of eliciting more specific data on that sexual attraction:

- In what ways did you find children sexually attractive?

Eric was the only man in this sub-sample to respond to these questions. He recalled that he found his victim sexually attractive. He enjoyed the physical contact with her, in particular, the 'closeness' and body shape, and was sexually aroused by her: 
'Feeling very close, that's the body side of things. Of cuddling rather than the face, you know the shape the body was, the closeness'.

\section{Becoming an abuser - further explored}

Here, the focus is on the men's accounts of becoming an abuser. In an attempt to 'understand' and interpret their accounts, a number of frameworks are imposed on their narratives, including: Finkelhor's (1984: 37) work on the four factors which contribute to the 'making of a child molester'. Moreover, in an attempt to detect further patterns in the men's accounts, they are ordered according to the presence of Core ITs (Ward and Keenan 1999: 836).

As previously noted, the 5 Core ITs relevant to child sexual abusers’ thinking are:

children as sexual objects

entitlement

dangerous world

uncontrollability

nature of harm

Table 8.8 summarises the extent to which Core ITs were present in the men's narratives.

Table 8.8: Core ITs evident in narrative?

$(\mathrm{N}=5)$

OFFENDER

CORE IT

\begin{tabular}{|l|l|l|l|l|l|}
\hline & $\begin{array}{l}\text { Children as } \\
\text { Sexual } \\
\text { Objects }\end{array}$ & Uncontrollability & $\begin{array}{l}\text { Dangerous } \\
\text { World }\end{array}$ & Entitlement & $\begin{array}{l}\text { Nature of } \\
\text { Harm }\end{array}$ \\
\hline ANDREW & Y & Y & N & N & N \\
\hline BILL & Y & Y & Y & N & N \\
\hline COLIN & Y & N & Y & N & Y \\
\hline ERIC & Y & Y & N & N & N \\
\hline GARETH & Y & Y & Y & N & Y \\
\hline
\end{tabular}


All 5 men perceived 'Children as Sexual Objects'. Four referred to the extent to which factors outside their control propelled them towards sexual activity with a child ('Uncontrollability'). Moreover, 3 referred to having been drawn to the safety of the child's environment ('Dangerous World'). There was evidence of the IT 'Nature of Harm' in only 2 men's narratives - those of Gareth and Colin. Gareth made a distinction between the abuse he perpetrated in a 'safe environment' and the more harmful abuse perpetrated by other sexual offenders. Colin believed that had his abuse been more serious, his victims would have 'fought against it'. It is interesting to note that the concept of 'Entitlement' did not feature in any of the men's accounts.

In the section which follows, the presence of Core ITs in the men's narratives are explored in further detail, beginning with the extent to which it is evident that they perceive 'Children as Sexual Objects'.

\section{CHILDREN AS SEXUAL OBJECTS}

Andrew saw the abuse he perpetrated against his 2 step-daughters as 'just a loving thing'. He placed responsibility for the abuse on his victims who were: 'putting [him] under pressure'. In Andrew's view, his victims 'asked for cuddles and kisses' and 'liked' what he did:

'The girls were so loving and caring but also egged it on. I'd be in bed on a Sunday and they'd get in and cuddle me and I'd get aroused sexually. It started through having a cuddle because they were very fond of me. The girls took advantage of that - they asked for cuddles and kisses...They knew what was happening. They liked me to do it. They'd say, do it again'.

Bill was the only man in this sub-sample who was undertaking the SOTP. It is unsurprising, therefore, that he showed some insight into his CDs - and the way in which he dealt with internal inhibitions (Finkelhor 1984) - in particular, the extent to which he gave himself permission to abuse his victim, who, in his view, 'enjoyed' the abuse:

'I convinced myself that she [victim] liked me with more than just affection. She was sort of sexually aware of things; she wanted me to experiment with her or whatever you might call it. I gave myself permission to abuse her, to masturbate her and so on. I convinced myself that she wanted this, she liked it. I wasn't 
forcing her to do anything. I wasn't forcing her to allow me to do what I was doing. She enjoyed it, she liked it, she wanted it, you know those sort of cognitive disorders. But that made it OK.'

Having abused his victim once, any concerns Bill had were subsequently allayed and justifying the abuse became easier. He believed that he was teaching his victim about sex:

'Each time I abused her it got easier. I was able to put the concerns to one side; pretending that it was OK. It was easier in the sense that I hardened my conscience, chose to ignore everything in me that said that it was wrong. It was a sort of a morbid curiosity. There was an element of experimentation there and exploration. This was obviously the first time for this particular girl, so in a sense I was kidding myself that I was teaching her something, introducing her to all these sexual things.'

Eric did not see his victim as a child:

'I'm not thinking of her as a child. I'm thinking about what I have done over the years in courtship. It was the natural reaction what I have done over my life with a woman'.

Gareth's narrative is permeated by the 'belief' that children have knowledge which enables them to make informed decisions about engaging in sexual activity. He emphasized there was no coercion involved - his victims consented. In his view, there was mutual agreement to the sexual activity:

'I asked them [victims] if I could touch them and they said yes. I touched them and that was it - conversation over with. I never touched any of my victims without consent. I never did anything to my victims that any of my victims didn't want me to do. I didn't see them as victims. I saw them as people with whom I was embarking upon a slightly sexual relationship. They were OK about it and I was OK about it and that was it'.

Gareth 'enjoyed' sexual contact with children; and expressed some satisfaction that the abuse he perpetrated had remained hidden for so long:

'I am a paedophile. I enjoyed contact with children in a sexual way. That is what I did for 12 years and I thought I was pretty damn good at it because I got away with it for 12 years'. 
Colin also 'enjoyed' sexual contact with children. Indeed, he explained that his sexual attraction to children had been ever present: 'all my life'.

Having explored the extent to which the IT 'Children as Sexual Objects' was evident in offenders' narratives, attention now shifts to their perceptions of their offending behaviour as being outside their control.

\section{UNCONTROLLABILITY}

A sense of 'Uncontrollability' was evident in 4 of the 5 men's narratives: Bill, Gareth, Eric and Andrew.

Prior to his offending, Bill's life was spiralling out of control; and 'had gone bad'. Indeed, 'nothing was taboo'; and 'madness' prevailed. During that time he recalled having:

'lots of idiot friends. It was dealing, doing drugs - all sorts of stuff, involved in prostitution as well. Basically I had just got myself into a very promiscuous sort of lifestyle, where anything goes, nothing was taboo. There was group sex, there was exploring the boundaries of sexual experimentation with other adults You know, straight, gay, anything basically and so the whole philosophy was just anything -nothing is taboo, nothing is sacred. So with that sort of philosophy and the sort of drunken lifestyle I was living: I wasn't drunk when I committed my offence but away from this girlfriend's, the rest of the time I was off getting drunk, with my friends, going to night clubs, just generally sleeping around with women. It was just then you start sliding sort of thing, it's almost like a drug. You just get yourself onto a roller coaster and you can't get off sort of thing. I don't expect that to make an awful lot of sense. It was a madness really.'

Parallels can be drawn here with the account of one of the male victims of femaleperpetrated CSA - recruited from the general population - in Deering and Mellor's (2011) study. That man's life was also characterized by a hedonistic approach and casual sex with females following the abuse he experienced. 
The extent to which Bill felt that his offending was beyond his control is further illustrated by the fact that he recognized that, given time, the abuse could - and probably would - have progressed:

'Given enough time, it is quite possible that the extent of the abuse could have gone further'.

As noted earlier, Ward and Keenan (1999: 830) propose that: 'uncontrollable factors are thought to leave the offender with deviant preferences that he is unable to suppress or manage'. Amongst the factors relevant to this are: early experiences such as CSA. In Bill's view, factors additional to the CSA he experienced - in particular, the absence of stability and 'stable relationships' in his life - had contributed to his abuse of his girlfriend's 6 year old daughter:

'Obviously not being abused as a kid would have helped, but I think really for me the biggest thing, looking back I would say, is the lack of stable relationships. I wasn't close to my parents. They got divorced. I was being abused at home by one parent. I was at boarding school which has a very cold army lifestyle. I just shut myself off then, from getting close to people, because all I was doing was getting hurt emotionally. There was a lot of chopping and changing in my life. My dad's work caused us to travel a lot and so we were constantly uprooted and shifted somewhere else and then uprooted again. And in my early adult life I was constantly uprooting, changing. So I was just a rolling stone. I wasn’t anybody's. No stable relationship with anybody'.

As a result of the abuse Bill experienced from his mother; and the knowledge that a teacher at his boarding school was abusing younger pupils (he was not abused by the teacher), he believed that he normalised abusive behavior, and became 'accustomed' to it. He described how the teacher:

'was into young boys and he was sadistic as well. He was physically abusing them as well as sexually. He was routinely physically and sexually abusing boys and, of course, you don't realize at the time that this wasn't normal, you just lived in fear of him'.

Bill explained how several factors played a part in overcoming internal inhibitors to abuse (Finkelhor 1984), resulting in an inability to 'resist' offending: 
'Other things [as well as abuse] had happened in life in my childhood at boarding school, and a promiscuous lifestyle had all broken down barriers as well. When I was put into that situation [having access to the victim], an opportunity developed I wasn't able to resist or deal with'.

Hilton and Mezey (1996) note that perpetrators often tend to replicate their own abusive experience when abusing their victims. Howitt (1995: 61), too, draws attention to the reenactment process, involving the direct replication of abuse experienced in childhood. This, it is argued: 'is an attempt to deal with the confusion and stress generated' by CSA. Some of the men in this sub-sample made reference to the similarities between the abuse they had experienced and that which they perpetrated. Some parallels, for example, can be drawn between Bill's account and that of one of the men in Etherington's (1997: 113) study, who abused his victim 'in the same ways as his mother had abused him'. Here, Bill describes how the offences he committed were 'very similar' to the abuse he experienced as a child:

'When I was abused as a kid, sexually abused, very similar to what I did to my victim was what was done to me as a small boy. It was by my mum - she masturbated me basically, from as early as I can remember'.

Andrew also made the connection between his own victimization and the abuse he subsequently perpetrated:

'If I'd known at a younger age what happened to me was wrong it'd have helped me quite a bit. Every time I talk about it, it helps. It'd have stopped me doing what I did to my stepdaughters. My nan buggered everything up for me'.

Throughout his narrative, Andrew, like Bill, reflected on the similarities between his offending and the CSA he experienced:

'It started with my nan, with her putting her hand down the back of my trousers and I thought it was OK. I used to do it with my own daughter and I did it with the girls [victims]'.

Gareth was of the view that the abuse he experienced had been 'an interesting game' which he had been taught, and which he became addicted to: 
'I was 11 [when my sister abused me] and it was a couple of weeks after she stopped touching me that I touched my niece. Then when I was 14, it [the abuse] went outside the family to a friend of my mum's, her daughter. I touched her a couple of times, and then she moved and then I went back into the family again. It was going on like that and then when I was 17, I touched somebody and she said that hurts, and that was when I actually realized, what am I doing? And I tried to stop. But I just couldn't stop doing it. It is like an addiction or something'.

Like Bill, Eric recognized the risk he presented. He believed that the abuse he perpetrated would have progressed had his victim lived closer to him, thus providing him with more opportunity to offend. He recalled: 'I could have done it more if she lived nearer.' Situational stressors appear to feature in Eric's case (Finkelhor 1984), as he reported being 'under a lot of stress' due to ill health; and of having 'gone through a rough time sexually' with his wife prior to offending. He was the only man in this group who reported resorting to alcohol before abusing his victim. He talked of: 'drinking before the offences were committed - a lot'; and of using alcohol to overcome the sense of wrongdoing he felt at offending, thus dealing with 'internal inhibitors' (Finkelhor 1984: 56):

'I did a lot of drinking during the day which seemed to kill off whatever it was that you were doing wrong - you know, something in you saying you are doing wrong now and again’.

\section{DANGEROUS WORLD}

The IT 'Dangerous World' was evident in 3 of the men's narratives: those of Bill, Gareth and Colin.

Bill met his victim's mother through the 'personal ads'. The victim's family was extremely vulnerable: her older sister had previously been sexually abused by her father. Bill's account provides an illustration of the part played by emotional congruence, or the ““fit” between the adult's emotional needs and the child's characteristics'; sexual arousal; and blockage, in motivation to abuse (Finkelhor 1984: 38). Bill described how he had been drawn to his victim, explaining that she 'was accepting' of him and met his emotional needs: 
'She liked me, she was accepting of me. She sort of emotionally bonded with me. My offence was as much emotional as it was sexual. I was getting emotional love and what I considered love you know: nice feelings when I was with kids because obviously they are unassuming and they are accepting. I was very conscious of the fact that I had made a bit of a mess of my life at that point: they [children] are quite accepting, forgiving.

It appears that although other sources of sexual gratification were available to Bill at the time he perpetrated abuse, he did not find these wholly satisfying; hence his attraction to his victim. It is possible that Bill abused his victim as part of his search for something different (Finkelhor 1984), given his previous lifestyle:

'I was already at the time of my offence getting more sexual gratification with consenting adult women than you could shake a stick at. I think there was more of an emotional need there than a sexual one. The abuse that I did to my victim was sexual obviously and I don't deny that I was getting gratification out of it but it caused us to be emotionally bonded in my mind'.

He described his thought processes prior to the abuse, when he recognised that he was attracted to his victim. Again, the emphasis here is on an emotional, as well as sexual, attraction:

'So once I got in a relationship with her mother, for the first two months or so nothing improper happened with the daughter but looking back now, I can see now that there was a lot of thoughts going on in my head during that two month period. I didn't actually offend but mentally I was going off the track big time. So, that was the period where there was an attraction there. I think it was an emotional attraction as much as sexual. I got on well with her [victim] and she liked me. I am just trying to explain how I was thinking at the time. She took a shine to me and came up to cuddle me the way kids do.'

Gareth, too, was emotionally drawn to children. He appeared to be 'blocked' from engaging in appropriate sexual relationships. Some research indicates that sibling abuse might impact on the victim's capacity to connect with their peers (Welfare 2008). Gareth's account appears to confirm this. He described how his sexual attraction to children was based on the affection they showed him, and his inability to relate to - and 'fit in' with - his peers. Like Bill, he talked of appreciating the accepting nature of his victims: 
'It's not just any children, it's specific children. All of my victims showed me a great affection. They were always happy to see me. It was always, are you going to play a game with us? Are you going to tell us a story? They just wanted to be with me. It was nice to be wanted. I like kids, I love kids. I get on better with kids than I do with people of my own age. I don't fit in with people my own age. I am not cool, I never have been. So I go to people who accept me for what I am. So I spent a lot of time hanging round with my nieces and nephews and with their friends'.

Colin clearly viewed the 'world as a dangerous place' (Ward and Keenan 199: 829). He explained that his attraction to children emanated from their 'innocence' in a 'god awful world'.

\section{NATURE OF HARM}

The Core IT ‘Nature of Harm’ was evident in only 2 men’s narratives - Gareth's and Colin's. There are some obvious differences between these men: Gareth was the youngest man in the sub-sample, at 25 years of age; and Colin the oldest, at 76 years. Yet, they share some characteristics. As noted earlier, both could be defined as fixated offenders; and both had abused multiple victims.

Gareth was confident that his offences had been committed in a 'safe environment' and was keen to make the distinction between his strategy and that of a stereotypical sexual predator:

'I have committed offences but I have committed them in a safe, permanent environment where the children felt they were safe. I didn't cause no stress by grabbing them off the streets away from their family or anything like that'.

Colin also believed that he had caused no harm to his victims who, in his view:

'could have fought against it [the abuse] had it been anything serious'.

He did not view his behaviour as sexually abusive; and his narrative was characterised by a total lack of remorse. 


\section{Summary: Part 2}

The majority of men in this sub-sample had abused either 1 or 2 victims. One man, however, (Gareth) had abused 12 victims. All 5 men had abused female victims, This finding is consistent with other research (Briggs 1995; Romana and DeLuca 1997)), which suggests that there is a similarity between the gender of those who abuse offenders and their later victims. Two men abused on an intra-familial level; 2 on an extra-familial level; and one abused across relationship boundaries (although, in this case the majority of victims - 10 - were intra-familial). Two men (Gareth and Colin) appear to fit the criteria for fixated offenders. A further 2 men (Bill and Andrew) referred to replicating some aspects of their abuse. Of note here, is that the abuse they experienced lasted longer than that experienced by the other men; and was perpetrated by adult females (a mother and grandmother) whose role is usually associated with caretaking and nurturing.

It is clear from Table 8.8, that all of the men perceived 'Children as Sexual Objects'. Four referred to the extent to which factors outside their control propelled them towards sexual activity with a child ('Uncontrollability'). Moreover, 3 referred to having been drawn to the safety of the child's environment ('Dangerous World'). There was evidence of the IT 'Nature of Harm' in only 2 men's narratives - those of Gareth and Colin. Gareth made a distinction between the abuse he perpetrated in a 'safe environment' and the more harmful abuse perpetrated by other sexual offenders. Colin believed that had his abuse been more serious, his victims would have 'fought against it'. It is interesting to note that the concept of 'Entitlement' did not feature within any of the men's accounts.

Scant attention has been paid to the impact of female-perpetrated CSA on males, particularly male child sexual abusers. Where research has been undertaken, samples have often included both male and female victims - drawn from the general population and the focus has been on maternal abuse, with little emphasis on abuse perpetrated by other females. However, as Bourke et al. (2014: 778) note, it is only through acknowledging and furthering understanding of female perpetrated CSA that the provision of: 'informed evidence-based prevention and education programmes, and 
appropriate treatment and assessment procedures' will be ensured. Notwithstanding the limitations of such a small sample, the research findings reported here have, to some extent, contributed to the knowledge base on this topic. By focusing on the individual experiences of 5 male child sexual abusers, some understanding has been gained of the heterogeneous nature of this form of offending, and its impact on a specific population.

In the following Chapter, attention shifts to the 3 men who were abused by both males and females in childhood. 


\section{Chapter 9: Research Findings - Experiencing male and female-perpetrated CSA}

\section{Introduction}

In the previous Chapter, the emphasis was on 5 out of 40 men who reported having been sexually victimised solely by females in childhood. In this Chapter, the focus is on an even smaller sample: 3 men who had been sexually abused by both males and females at different points in their childhood. Thus, there are limitations to the findings which are presented here. The emphasis once more, is on the men's experiences of abuse; their perceptions of that; its impact on them; and the extent to which it led to them 'becoming an abuser'. The men's experiences and perceptions of their childhood sexual victimisation are presented in Part 1 of this chapter. Their account of 'becoming an abuser' follows, in Part 2. Of note, is that these 3 men appeared more reluctant to talk about aspects of their own abuse and subsequent offending than many of the other men in this study. Two (David and Frank) appeared to be much more 'comfortable' discussing the male-perpetrated abuse they had experienced than abuse by a female. The possible reasons for their reticence are explored in the Discussion.

Consistent with the approach adopted throughout this thesis, the profiles of all 3 men are examined at the outset of this chapter. Qualitative data collected during in-depth, individual interviews are then drawn on. In presenting the men's accounts, once more, their names have been changed; and some details omitted in order to preserve anonymity. 
Part 1: Offenders' experiences and perceptions of male and female-perpetrated CSA The 3 offenders

Table 9.1: Demographic characteristics

\begin{tabular}{lll}
\hline$(\mathrm{N}=3)$ & & \\
Age: & $31-45$ & 2 \\
& $46-55$ & 1 \\
Marital status: & married & 1 \\
& divorced & 2 \\
Children: & 2 & 2 \\
& 3 & 1 \\
Qualifications: & & 3
\end{tabular}

Offenders in this sub-sample ranged in age from 38 to 55 years at the time of interview. All 3 were, or had been, married; and all had children of their own. None of the men in this group had any qualifications.

Table 9.2: Offending behaviour

$(\mathrm{N}=3)$

Previous offending:

Current sentence: sexual offences (adults) theft/fraud

$>4$ years
No. offenders

2

1

3

Two men in this group had previously been convicted of sexual offences against adults: namely, rape of a female adult; and indecent assault. They had each served a prison sentence for those offences. However, neither of these men could be defined as specialists (Harris et al. 2009), as they each had convictions for a range of other non-sexual offences. The most recent offences committed by the men were located towards the more severe end of the continuum of sexual offending. All 3 had received prison sentences which were 4 years and over in length. One of the men in this group, David, was undertaking the SOTP at the time of interview. 
In the section which follows, information is presented on the number, age and gender of offenders' victims; and also the duration of abuse. This information has not been routinely collected in research with child sexual abusers. Given this, the aim here is to provide as detailed a picture as possible of the men's offending.

Table 9.3: Victims + duration of abuse

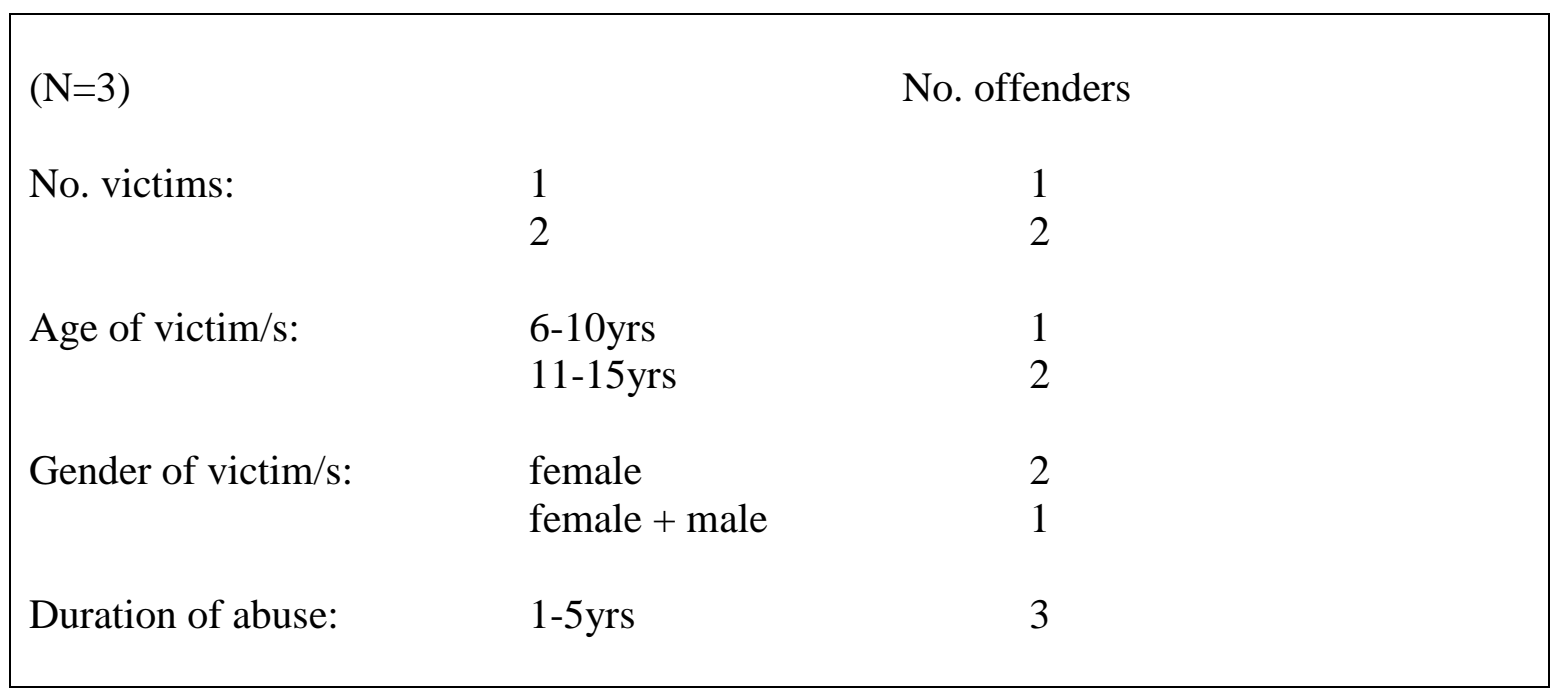

The majority of offenders had abused either 1 or 2 victims. Those victims ranged in age from 8-14 years. Two of the men abused as fathers; and one as both grandfather and father. All abused their victims in their own home (this was often also the victims' home). David and Frank perpetrated abuse over a 4 year period. Both had ready access to their victims - the former abused his daughter, and the latter both his daughter and granddaughter - and this enabled the abuse to be facilitated and maintained across time.

Table 9.4 provides further detail on the men's offences; and their relationship to their victims.

Table 9.4: Offence/s \& offenders' relationship to victim/s

\begin{tabular}{|l|l|l|l|}
\hline $\begin{array}{l}\text { Offenders: } \\
(\mathrm{N}=3)\end{array}$ & $\underline{\text { Offence/s: }}$ & $\frac{\underline{\text { Relationship to }}}{\underline{\text { victim/s }}}$ & Relationship (contd.) \\
\hline David & Incest & Daughter (13 years) & Intra-familial \\
\hline Frank & Indecent assault & $\begin{array}{l}\text { Daughter \& } \\
\text { Granddaughter (both } \\
\text { at 13 years of age) }\end{array}$ & Intra-familial \\
\hline Hugh & $\begin{array}{l}\text { Attempted rape; USI; } \\
\text { buggery }\end{array}$ & $\begin{array}{l}\text { Daughter (10 years) } \\
\text { \& son (8 years }\end{array}$ & Intra-familial \\
\hline
\end{tabular}


There are a number of interesting issues to note about these men's offending. All 3 offended against children who were biologically related to them; all were fathers to their victims. The abuse perpetrated by Frank, however, was tri-generational, involving first his daughter, and then his granddaughter. In David and Hugh's case, the abuse they perpetrated was penetrative in nature. These, and other issues of relevance to these men are examined in further detail later in this Chapter. Attention now turns to their reported childhood sexual victimisation. First, their relationship to their perpetrators is examined.

Table 9.5: Offenders' childhood sexual victimisation relationship to perpetrator

$(\mathrm{N}=3)$

Perpetrators' relationship to offender:

Offender:

Sister + brothers $(\mathrm{I} / \mathrm{F})$

HUGH

Family friend + male residential careworker $(\mathrm{E} / \mathrm{F})$

DAVID

Schoolfriend's mother + headmaster $(\mathrm{E} / \mathrm{F})$

FRANK

Key: I/F - intrafamilial; E/F - extra-familial.

All 3 men in this sub-sample had been re-victimised in childhood; and were sexually abused by both males and females. One man (Hugh) had been abused by his brothers as well as his sister. The other 2 men reported abuse by females - a family friend and schoolfriend's mother; and also by males within institutional settings. One man (David) had also been abused by a residential care worker; and the other (Frank) by the headmaster at an approved school.

Each of these men's childhoods were also characterised by physical violence, meted out by their father; stepfather; and mother and stepfather in one case. Each had a previous conviction: 2 of the men had previously been convicted of sexual offending. In 2 cases, the abuse they subsequently perpetrated was penetrative in nature. Moreover, all abused intrafamilial victims over significant periods of time, with Hugh abusing both male and female victims.

Having provided some detail of offenders' backgrounds; their offending behaviour; and the CSA they experienced, attention now shifts to the men's accounts of that abuse; their 
perceptions of it; and their subsequent sexual offending. Once more, these accounts are presented verbatim and are ordered according to the 3 themes below, which emerged from analysis of the interview data:

- Offenders' experiences of male and female-perpetrated child sexual abuse

- Offenders’ perceptions of male and female -perpetrated child sexual abuse

- Becoming an abuser

The men's experiences and perceptions of their childhood sexual victimisation are presented first. Their account of 'becoming an abuser' follows. Again, in line with the approach followed throughout this thesis, an attempt is made to tease out difference where that may exist.

\section{Experiencing intra-familial CSA - male and female perpetrators}

Only one of the 3 men in this sub-sample had been abused on an intra-familial level in childhood. Hugh, like Gareth, had experienced sibling abuse. Hugh was 43 and divorced at the time of interview. He had previous convictions for theft, for which he was fined; and rape against an unknown adult female, for which he had received a 4 year prison sentence. He had been convicted of attempted rape and USI against his daughter; and buggery against his son and daughter. The offences were committed in Hugh's (and the victims') home. His son was aged 8 , and his daughter 10 . Hugh reported having been sexually abused from the age of 12 by his sister and brothers. He also reported having been physically abused as a child by his father. Hugh referred to the sexual activity with his sister as 'play' as opposed to abuse:

'My sister played with me and my 2 younger brothers. She simulated sex with me at 12 '.

He was reluctant to talk in any further detail about his CSA. However, he did report, like so many other sexual offenders, that his early life was also characterized by physical violence and fear. He had been physically abused as a child by his father who 'used to get carried away at times' and recalled his relationship with his parents as 'non-existent':

'My father was very strict - I'm still scared of him. My father used to drink and fight'. 


\section{Experiencing extra-familial CSA - male and female perpetrators}

The 2 remaining men, Frank and David, had experienced extra-familial abuse. Their cases are distinguished by 2 separate experiences of sexual victimization in childhood.

Frank was 55 and married at the time of interview. He had a previous conviction for indecent assault against an adult female, for which he received a prison sentence. He had been convicted of indecent assault against his daughter and also his granddaughter. Both were aged 13 at the time of the offences; and both had been abused at Frank's (and one of his victims') own home. The abuse perpetrated against his daughter had lasted for 4 years and against his granddaughter, 2 years. Frank reported having been abused in childhood by his schoolfriend's mother and later, by the headmaster at an approved school. Frank had also been physically abused in childhood by the headmaster.

The abuse by his schoolfriend's mother began when Frank was 12:

'My schoolfriend's mother used to expose herself to me. I would call to X to go to school and he was always late getting ready. She would sit there with her dressing gown on, no undies on. And then she would come over and open the curtains and then you could see most of her breasts and then she started feeling, touching you. But it went a lot further than that. She would masturbate me. To start with, she would move the chair close to the table and then she would grab the table to pull it forward and then she would be in my crutch and that'.

At 14, Frank was sent to an approved school 'for breaking and entering':

'The approved school - I think it was about 12 months or 2 years depending on your behaviour. Because I was abused, I ran away from there and then I got into more trouble and then they changed it to a Borstal sentence'.

He described how, before running away, he had been raped three times by the headmaster:

'Well apparently, it happened to a lot of boys there. You would get picked to go into a special room, which you thought you were having a privilege. Only he would come in the night then and jump into bed with you. We was in dormitories and there was these two rooms then, that was if you had special privileges, but that was for his privileges. I stuck it for a week after it was happening - it happened 3 times - and then I run away from there'.

David was 38 and divorced when interviewed. He had been convicted of incest against his daughter who was 14 when the abuse began. The abuse was perpetrated at the family home over a 4 year period. David reported two separate experiences of CSA involving first, a male 
(a residential care worker); and then a female (his mother's best friend). David was also physically abused in childhood by his step-father.

Much of David's childhood was spent in residential care; an environment in which he experienced CSA:

'I grew up mostly in children's homes, from when I was 5 or 6 to 16. I don't know who my dad is. My mum always had men at home. My first recollection of sexual abuse was at 7. It was a staff member at the cottage home. It lasted a couple of months. He tried to get me to masturbate him and tried to bugger me once'.

David was also sexually abused by his mother's best friend at the age of '13 or 14':

'I was abused by my mum's best friend. It was a full sexual relationship - I was 13 or 14 at the time. She was 27. It ended when we moved house'.

\section{Categorizing the abuse experienced}

Notwithstanding the limitations of this research - in particular, the small sample size - these men's accounts provide some insight into childhoods which were characterised by sexual and physical violence from a young age. The CSA they experienced ranged from: exposure; to masturbation; simulated sex; and full sexual intercourse. Table 9.6 summarises the nature of that abuse; provides information as to the age of onset and duration; and interprets the men's abusive experience with reference to Lawson's (1993) categories of abuse - subtle; seductive; perversive; overt; and sadistic. 
Table 9.6:Categorizing abuse

\begin{tabular}{|c|c|c|c|c|c|}
\hline $\begin{array}{l}\text { Offender: } \\
(\mathrm{N}=3)\end{array}$ & Abused by: & $\begin{array}{l}\text { Nature of } \\
\text { abuse: }\end{array}$ & $\begin{array}{l}\text { Form of } \\
\text { abuse: }\end{array}$ & $\begin{array}{l}\text { Age at onset } \\
\text { of abuse: }\end{array}$ & $\begin{array}{l}\text { Duration of } \\
\text { abuse: }\end{array}$ \\
\hline David & $\begin{array}{l}\text { Mother's best } \\
\text { friend } \\
\text { (\& male } \\
\text { member of } \\
\text { staff at } \\
\text { residential } \\
\text { home) }\end{array}$ & $\begin{array}{l}\text { FSI } \\
\text { CWP \& } \\
\text { CAP } \\
\text { (attempted) }\end{array}$ & Overt & $\begin{array}{l}13 \text { years } \\
7 \text { years }\end{array}$ & $\begin{array}{l}2 \text { years } \\
2 \text { months }\end{array}$ \\
\hline Frank & $\begin{array}{l}\text { Schoolfriend's } \\
\text { mother } \\
\text { (\& } \\
\text { headmaster at } \\
\text { approved } \\
\text { school) }\end{array}$ & $\begin{array}{l}\text { E \& M } \\
\text { CAP }\end{array}$ & $\begin{array}{l}\text { Seductive \& } \\
\text { Overt }\end{array}$ & $\begin{array}{l}12 \text { years } \\
14 \text { years }\end{array}$ & $\begin{array}{l}2 \text { years } \\
1 \text { week (ran } \\
\text { away) }\end{array}$ \\
\hline Hugh & $\begin{array}{l}\text { Sister } \\
\text { \& brothers }\end{array}$ & $\begin{array}{l}\text { SS } \\
\text { N/K }\end{array}$ & Overt & 12 years & $\mathrm{N} / \mathrm{K}$ \\
\hline
\end{tabular}

[Key - Female-perpetrated CSA:

FSI = full sexual intercourse; $M=$ masturbation; $E=\operatorname{exposure;~} S S=\operatorname{simulated~sex;~} F=$ fondling

Key - Male-perpetrated CSA:

CAP $=$ contact abuse with penetration

CAWP = contact abuse without penetration (Erooga et al. 2012)

N/K = unknown (offender did not wish to discuss nature of abuse)]

It appears that all of the men experienced overt abuse: that is, abuse characterised by 'overtly sexualised contact' (Lawson 1993: 266). This form of abuse ranges from intercourse; to fondling of the child's genitals; coercing the child to fondle the female's breasts or genitals; cunnilingus; fellatio; digital penetration (or penetration with objects) of the child's vagina or anus; and sexualized touching and kissing (Saradjian 1996). One man, Frank, also experienced seductive abuse: behaviour which may include seductive and sexual posing; and nudity, as a precursor to an overt form of abuse.

As already noted in Chapter 2, Denov (2003), more recently classified female-perpetrated abuse - based on its severity - as follows: 
Severe sexual abuse - for example, sexual intercourse; penetration (anal or vaginal) with objects; or digital penetration; fellatio; and cunnilingus

Moderate sexual abuse - for example, genital fondling (without penetration); and simulated intercourse

Mild sexual abuse - for example, sexualized kissing

It appears that the men in this sample experienced what Denov (2003) refers to as severe or moderate sexual abuse.

\section{Disclosing abuse}

Where victims are abused by both male and female perpetrators it is more likely that they will reveal abuse by the former: 'long before revealing information about being molested by a female' (Briggs 1995: 168). Frank was the only man in this sub-sample to have disclosed abuse in childhood: albeit the male-perpetrated, as opposed to the female-perpetrated abuse he experienced. Although children abused by professional perpetrators are less likely to disclose than other victims (Roberts and Vanstone 2014), Frank did attempt to tell of the abuse perpetrated by the headmaster at an approved school. He recalled his distress at the response of staff; and realized, to his dismay, that they had been aware of the abuse but were not prepared to act. The extent of his pain and discomfort was dismissed; and he was humiliated by his peers' response. It appears that his sense of helplessness (Summit 1983) might have been exacerbated by the level of collusion and 'united front' (Spiegel 2003: 248) presented by abusing and non-abusing staff at the home. Subsequently, he felt that his only option was to run away:

'He [headmaster] put his penis up my backside 3 times. The first time, I had to go to the nurse because I was bleeding but it was a male nurse and all I got was a bit of cream. They knew what was going on but nothing was being done about it. And, of course, the other boys would be sniggering then. They would see you coming from the nurse and the way that you was walking, so they knew what was happening to you. It didn’t happen the second night but the third and fourth night it did and then, by the end of the week, I had run away from there'.

Evidence suggests that victims of female-perpetrated abuse encounter a more profound sense of isolation (Deering and Mellor 2011) thought to result from the 'silence' surrounding this 
form of abuse. Those abused by siblings are less likely to disclose than other victims (Stathopoulos 2012). It is not surprising, therefore, that Hugh who had been abused by his sister, like Gareth, did not tell. Perhaps David's experience with his mother's best friend falls into the category of initiation into sexual activity; thus disclosure was inhibited there.

Having explored offenders' experiences of male and female-perpetrated CSA, and the extent to which they disclosed that abuse, attention now shifts to their perceptions of CSA and its impact on them. 


\section{Offenders' perceptions of male and female-perpetrated CSA}

Table 9.6, provides information on offenders' childhood experience of sexual victimisation; their perceptions of that abuse; and their subsequent sexual offending in adulthood. Of interest, is that one man, Frank, had mixed views of the female-perpetrated abuse he experienced, citing a resulting sense of 'fear and excitement'. The 2 remaining men - both of whom had been abused by a female and then a male - provided no detail as to how they viewed their abuse.

Table 9.6: Offenders' perceptions of their childhood sexual victimisation + details of their subsequent offending

$(\mathrm{N}=3)$

\begin{tabular}{|c|c|c|c|c|c|}
\hline & Abused by: & $\begin{array}{l}\text { Nature of } \\
\text { abuse: }\end{array}$ & $\begin{array}{l}\text { Viewed } \\
\text { abuse: }\end{array}$ & Offence/s: & Victim/s: \\
\hline David & $\begin{array}{l}\text { Mother's best } \\
\text { friend - from } \\
13 \text { years of age } \\
\text { ( } \& \text { male } \\
\text { member of } \\
\text { staff at } \\
\text { residential } \\
\text { home) }\end{array}$ & $\begin{array}{l}\text { FSI } \\
\text { CWP \& } \\
\text { CAP } \\
\text { (attempted) }\end{array}$ & $\begin{array}{l}\mathrm{N} / \mathrm{K} \\
\mathrm{N} / \mathrm{K}\end{array}$ & Incest & $\begin{array}{l}\text { Daughter (13 } \\
\text { years) }\end{array}$ \\
\hline Frank & $\begin{array}{l}\text { Schoolfriend's } \\
\text { mother - from } \\
12 \text { years of age } \\
\text { (\& } \\
\text { headmaster at } \\
\text { approved } \\
\text { school) }\end{array}$ & $\begin{array}{l}\text { E \& M } \\
\text { CAP }\end{array}$ & $\begin{array}{l}\text { Mixed - } \\
\text { fear \& } \\
\text { excitement } \\
\mathrm{N}\end{array}$ & $\begin{array}{l}\text { Indecent } \\
\text { assault }\end{array}$ & $\begin{array}{l}\text { Daughter \& } \\
\text { granddaughter } \\
\text { (13 years) }\end{array}$ \\
\hline Hugh & $\begin{array}{l}\text { Sister \& } \\
\text { brothers - } \\
\text { from } 12 \text { years } \\
\text { of age }\end{array}$ & SS & $\mathrm{N} / \mathrm{K}$ & $\begin{array}{l}\text { Attempted } \\
\text { rape; USI; } \\
\text { buggery }\end{array}$ & $\begin{array}{l}\text { Daughter (10 } \\
\text { years) \& son } \\
\text { (8 years) }\end{array}$ \\
\hline
\end{tabular}

[Key - Female-perpetrated abuse:

FSI = full sexual intercourse; $M=$ masturbation; $E=\operatorname{exposure;~} \mathrm{SS}=\operatorname{simulated}$ sex; $\mathrm{F}=$ fondling

Key - Male-perpetrated abuse:

CAP = contact abuse with penetration

CAWP = contact abuse without penetration (Erooga et al. 2012)

$\mathrm{N}=$ Negative perception of abuse; N/K = unknown (offender did not wish to discuss)]

Frank was the only one of the 3 men who had been abused by a female and male in childhood to reflect on his perceptions of female-perpetrated abuse. The remaining 2 men - David and 
Hugh - were much more reluctant to talk about their experience. Frank had been abused by his schoolfriend's mother when he was 12 years of age. He recalled how he viewed the abuse with a mixture of fear and excitement; and as a 'part of growing up'. He was clearly relieved that he never found himself alone in the house with his abuser:

'I was lucky in a way that the younger brother started school at the same time then and he was up early and there was always one of them there. But when I look back now, I think that maybe it was a part of growing up. But then you know, I was afraid in one way but excited in another'.

Frank had also been sexually abused by the headmaster at an approved school; his perception of that abuse was wholly negative, resulting in him running away. Of course, the nature of that abuse was more intrusive, involving anal penetration, whereas the female-perpetrated abuse he experienced involved exposure and masturbation. Nevertheless, it raises questions as to the distinction between perceptions of female-perpetrated abuse and that perpetrated by males. This issue is further examined later in the thesis, in the Discussion.

\section{Summary: Part 1}

In this Chapter, the focus is on 3 men - David, Frank and Hugh - who reported having been sexually abused by both males and females in childhood. In Part 1, their accounts of that abuse; their perceptions of it; and its impact on them has been explored.

These 3 men share some characteristics, for example: all were, or had been married; and all had children of their own. Two (Hugh and Frank) had previous convictions for sexual offences against female adults: rape and indecent assault, respectively. Each had served a prison sentence for those offences. All 3 men offended on an intra-familial level: two men abused as fathers (David and Hugh) and the third (Frank) as father and grandfather. David and Frank abused female victims; and Hugh both male and female victims.

There are a number of factors which may have increased these men's vulnerability to CSA. All 3 were subjected to physical violence in childhood; and all experienced sexual revictimisation, and abuse by males and females: Hugh by his sister and brothers from the age of 12; David and Frank, by a known female and a male in a position of trust. The childhoods of all 3 men were highly sexualised. Hugh's abuse by his siblings occurred in a family environment characterised by a 'non-existent' relationship with his parents, and fear of a 
violent father who engaged in alcohol abuse. Frank's childhood was marked out by femaleperpetrated abuse at the age of 12 . His offending, at 14, led to a period in institutional care, where he was also abused. David was first abused by a male in a position of trust, at the age of 7 ; and then by a female when aged ' 13 or 14'. David's home environment had been characterised by his mother's sexual activity with men. As a child, he had spent much of his time in residential care. Both Frank and David experienced penetrative CSA; the former, from a male and the latter, from a female.

Only one man, Frank, disclosed abuse in childhood, albeit the male as opposed to the femaleperpetrated abuse. The response he received, from both staff and his peers, resulted in his running away. Staff were aware of the abuse but failed to intervene; he was humiliated by the response of his peers, who were also aware that he had been abused. Frank was the only man to articulate his perceptions of the abuse he experienced. Of note on this, is the distinction between his views of male-perpetrated as opposed to female-perpetrated abuse. His view of the former was wholly negative, and as already noted, was demonstrated by his running away. However, he viewed the latter with a mixture of fear and excitement; and saw it as 'a part of growing up'. While there were differences in the nature of these abusive experiences which may account for differing perceptions, one cannot discount the impact of the gender of the perpetrator here: in particular, the extent to which female-perpetrated abuse is characterised as an initiation into the world of sexual activity for young males.

In Part 2 of this Chapter, attention shifts to the men’s accounts of becoming an abuser. 


\section{Part 2: Becoming an abuser}

\section{Introduction}

As noted previously, during interview, all the men were asked the following questions in order to determine their understanding of the origins of their sexual offending. The questions were designed to incorporate any abusive or negative experiences in childhood, both within and outside the family; and also to enable a focus on abusive or negative experiences with children and young people:

- To what extent do you see a link between your own family experiences as a child and your offences against children?

- To what extent do you see a link between your relationship with childhood friends, inside and outside school, and your offences against children?

- To what extent do you see a link between your relationship with any significant adult(s) outside your immediate childhood family and your offences against children?

\section{Making the link}

Only one man (Hugh) cited a link between childhood experiences and his later offending; and that, he felt, was 'minimal'. No further detail was provided on this.

In an attempt to determine awareness of when their sexual attraction to children emerged and whether perhaps offenders could be classified as either fixated or regressed - the men were also asked:

- How old were you when you first became aware that you were sexually attracted to children? 
Again, only one man - Frank - identified the age at which he became aware that he was sexually attracted to children: 44 . Prior to that, however, he had sexually abused his daughter, before, 18 years later, abusing his granddaughter.

In order to determine their understanding of the origins of their sexual attraction to children, offenders were also asked:

- How do you think you came to be sexually attracted to children?

And as a means of eliciting more specific data on that sexual attraction:

- In what ways did you find children sexually attractive?

Frank described how he first became sexually attracted to his daughter 'when she was developing' at ' 11,12 years of age'.

For David, the sexual attraction also related to his victim's physical attributes: 'the physical side of the female body'. 


\section{Becoming an abuser - further explored}

Here, the focus is on the men's accounts of becoming an abuser. In an attempt to 'understand' and interpret their accounts, a number of frameworks are imposed on their narratives, including: Finkelhor's (1984: 37) four factors which contribute to the 'making of a child molester'. Moreover, in an attempt to detect further patterns in the men's accounts, they are ordered according to the presence of Core ITs (Ward and Keenan 1999: 836).

As previously noted, the 5 Core ITs relevant to child sexual abusers' thinking are:

\section{children as sexual objects}

entitlement

\section{dangerous world}

uncontrollability

nature of harm

Table 9.8 summarises the extent to which Core ITs were present in the men's narratives. All 3 perceived 'Children as Sexual Objects'; and all referred to a sense of 'Uncontrollability' resulting from difficulties or stressors which, in their view, served as precursors to offending: namely, relationship and financial difficulties. Interestingly, none of the men referred to their own abuse as a contributory factor in their offending; and none of the other 3 Core ITs were evident in the men's narratives.

Table 9.8: Core ITs evident in narrative?

$(\mathrm{N}=3)$

OFFENDER

CORE IT

\begin{tabular}{|l|l|l|l|l|l|}
\hline & $\begin{array}{l}\text { Children as } \\
\text { Sexual } \\
\text { Objects }\end{array}$ & Uncontrollability & $\begin{array}{l}\text { Dangerous } \\
\text { World }\end{array}$ & Entitlement & $\begin{array}{l}\text { Nature of } \\
\text { Harm }\end{array}$ \\
\hline DAVID & $\mathrm{Y}$ & $\mathrm{Y}$ & $\mathrm{N}$ & $\mathrm{N}$ & $\mathrm{N}$ \\
\hline FRANK & $\mathrm{Y}$ & $\mathrm{Y}$ & $\mathrm{N}$ & $\mathrm{N}$ & $\mathrm{N}$ \\
\hline HUGH & $\mathrm{Y}$ & $\mathrm{Y}$ & $\mathrm{N}$ & $\mathrm{N}$ & $\mathrm{N}$ \\
\hline
\end{tabular}

In the following section, further detail is presented on the presence of ITs in the men's narratives, beginning with the extent to which they viewed 'Children as Sexual Objects'. 


\section{CHILDREN AS SEXUAL OBJECTS}

David, like Luke, had been convicted of incest against his 14 year old daughter; and there are some parallels evident in their narratives. David was undertaking the SOTP at the time of interview and demonstrated some understanding of certain aspects of his offending. He did not view his victim as a child and recalled:

'I didn't see her [victim] as a child'. Rather, she became a substitute for his wife who had left the marital home.

For David, the emotional element to his relationship with his daughter was based on the fact that he felt he could 'communicate' with her, and the sense in which they 'shared' household tasks. Of interest, is his reference to living together with his daughter - a phrase usually ascribed to adult relationships or partnerships:

'Her whole personality - we could communicate. Sharing all the tasks (household). The offences began 12 months after we lived together. Until then, my wife lived at home'.

Finkelhor (1984: 58) notes that: 'the influence of third parties appears as an important factor in creating a vulnerability to abuse', with mothers playing a central role in protecting children from abuse. Finkelhor argues that 'when mothers are incapacitated in some way, children are more vulnerable to abuse' (pp.58-59). Mothers may be unable to protect because of absence from the family, for example, due to divorce or ill-health. They may also be 'psychologically absent' (p.59) due to relationship difficulties with their husband or children.

In David's case, his wife had left the marital home and he subsequently cared for his daughter alone. The fact that he was: 'left alone in the absence of supervising persons', appears to have provided an opportunity to abuse; and assisted with the overcoming of external inhibitors (Finkelhor 1984: 60).

There is a dearth of research on abuse perpetrated by grandparents (Margolin 1992), with few cases of this form of abuse identified in the literature on sexual offending (Russell 1986: Finkelhor et al. 1984). Margolin (1992) draws attention to the gendered nature of this form of abuse, as most perpetrators are male and most victims female. In some cases, grandfathers will have already abused their own children - 'first generation victims' (Goodwin et al. 1983: 168) - before later moving on to abuse their grandchildren, who may be the daughter/s of 
their original victim. Some of these men, therefore, will have a lengthy history of offending (Margolin 1992). This is of relevance, as Frank’s abuse was tri-generational in character (Margolin 1992); he had abused his daughter and also his granddaughter.

Once more, there is evidence of the offender viewing the victim (his daughter) as an adult as opposed to a child:

'If she was bad she would come and sleep between me and the missus and I think that was when it first started. I was the father and she was the girl and we had always had a close relationship. I was thinking of her more as a woman than a child'.

He abused his daughter's child - his granddaughter - 18 years later. The majority of grandfather perpetrators in Goodwin et al.’s (1983: 166) research abused their granddaughters following a 'loss' of some sort, for example, bereavement or separation from their partner or spouse. While a sense of 'loss', in the form of marital problems, played a part in Frank's abuse of his daughter, he explained how he had been drawn to his granddaughter. She was the youngest of several grandchildren but he was not attracted to any of them. He believed that his granddaughter's resemblance to her mother (he recalled: 'she looked more like my daughter') had perhaps ‘triggered' an attraction:

'Her sister, being the older one, was a very big girl - well developed. And yet I had no attraction to her whatsoever and it was the same with my other granddaughters. I have got no attraction to them in that way at all. It was as if I was seeking and enjoying the attention that I was having off her [victim]'.

In Frank's view, the abuse he perpetrated was a 'loving' act:

'It was as if I was doing it lovingly, not trying to violate them or threaten them'.

There are some parallels between the abuse perpetrated by Frank and Hugh - although there are also some distinct differences. Hugh, like Frank, had abused more than one family member; and both had abused victims who were biologically related to them. Hugh, however, had abused his son and daughter, who were younger than Frank's female victims when the abuse began. As was the case with Gareth, Hugh's narrative is permeated by the 'belief' that children have sufficient knowledge to enable them to make informed decisions about engaging in sexual activity. This is interesting, as both men had experienced abuse perpetrated by siblings. Although, of course, Hugh's case is distinguished from Gareth's by the fact that he had experienced abuse perpetrated by his sister and also his brothers. 
According to Hugh, his victims consented to the sexual activity. He explained that he:

'just asked them [victims] and they agreed [to the sexual activity]'.

There are a number of issues to note on this. Of course, children do not have sufficient knowledge of sexual activity - and all that it entails - to consent; and in the UK, this is now clarified under the Sexual Offences Act 2003; second, requests to comply with sexual activity are more likely to be agreed to if the victim is in a close relationship with, and trusts the offender (Finkelhor 1984).

Having explored the extent to which the IT 'Children as Sexual Objects' is evident in offenders' narratives, attention now shifts to their perceptions of their offending behaviour as being outside their control.

\section{UNCONTROLLABILITY}

There is evidence of 'blockage' (Finkelhor 1984) in all 3 men's narratives. All 3 referred to marital difficulties as 'stressors'; and as a precursor to offending. Frank cited the abuse he perpetrated against his daughter as stemming from marital problems:

'With my daughter, it happened at a time that I was having problems with my wife. I don't blame my daughter in any way for what happened. I hadn't had sex and whether I was looking for affection off her and she was giving it to me or what, I don't know.'

Frank recognized what he was doing was wrong but, like several of the other men, felt unable 'to stop' the abuse:

'I did know what I was doing was wrong but I wasn’t strong enough to stop myself'.

Abuse may be enabled, for example, where family members share a room or bed (Finkelhor 1984). This is relevant in David's case; he talked of sharing a bath and bed with his daughter as a precursor to abuse. Here, situational factors appear to feature in the lowering of internal inhibitions, in particular, 'stressors' including relationship difficulties (Finkelhor 1984). In David's view, the breakdown of his relationship with his wife; and the fact that she subsequently left the family home, led to him offending against his daughter, who took the place of his wife in every sense of the word. He recalled:

'We lived in very close proximity. We were together for so long that I grew towards her. 
Like Eric, Hugh described experiencing ‘stress’ prior to the abuse. In Hugh's case this was due to financial difficulties. He also referred to experiencing depression; and, like David and Frank, marital problems:

'I was under a lot of stress because of the mortgage and finances and the wife kept on about teaching the kids the facts of life. So, I thought rather than tell them I'd show them. I got more and more depressed and our love life finished (mine and the wife's)'.

\section{Summary: Part 2}

In Part 2 of this Chapter, the focus was on the men's accounts of becoming an abuser. These men were more reluctant to discuss their sexual attraction to children than the men in the other 2 sub-samples; and they made little connection between the CSA they experienced and their subsequent offending. The extent to which Core ITs were present in the men's narratives was explored, and it was clear that all 3 perceived 'Children as Sexual Objects'. Moreover, all referred to a sense of 'Uncontrollability' resulting from difficulties or stressors which, in their view, served as precursors to offending: namely, relationship and financial difficulties. Interestingly, none of the men referred to their own abuse as a contributory factor in their offending; and none of the other 3 Core ITs were evident in the men's narratives.

In the following - and final - Chapter of the thesis, these findings and those relating to the other 37 men who participated in this research are discussed in further detail. Distinctions are made within and between all 3 groups of offenders referred to in the thesis, in order that difference - where that exists - may be explored and understood. 


\section{Chapter 10: Discussion and Conclusion}

\section{Introduction}

In this, the final Chapter of the thesis, the emphasis is on further discussion of - and concluding thoughts on - the research findings which emerged from this study. The findings are discussed with a particular emphasis on comparing and contrasting findings within and between the 3 groups of offenders. A 'model' of vulnerability is proposed, based on the men's accounts; and on what might be gleaned from those about the factors that might render some children more vulnerable to CSA, and some victims at increased risk of becoming an abuser. Moreover, the implications of the research for policy and practice with both victims and offenders are considered, as are the limitations of the study. In the first instance, the aims and objectives of the research; the research questions which served to guide the work; and the structure of the thesis are revisited.

\section{Aims and objectives of the research}

In undertaking the research, the aims and objectives were as follows:

1.To review the literature on the links between childhood sexual victimization and subsequent sexual offending and elicit gaps in understanding and knowledge.

2. To explore child sexual abusers' accounts of their own childhood sexual victimisation.

3. To explore their perceptions of that.

4. To explore their views on the impact of the abuse they experienced.

5. To explore their views on the extent to which their childhood sexual victimisation might have played a contributory part in their 'becoming an abuser'. 


\section{The research questions}

Previous research has drawn on quantitative methods, measures, and checklists to explore the problem of the link between victimisation and offending. In contrast, here, the emphasis has been on a phenomenological approach: on abusers' accounts of their pathway from victim to victimiser; and the meaning of that for them. The following research questions provided direction for the study. Each was designed to elicit difference within and between groups of offenders, and the questions are listed in full in the Introduction:

1.What are imprisoned, adult male child sexual abusers' experiences of sexual abuse in childhood?

2. What are their perceptions of the abuse they experienced?

3. What, according to offenders, is the impact of the abuse they experienced in childhood?

4. Did offenders disclose the abuse they experienced in childhood?

5. Are offenders' accounts consistent with the existing evidence on the link between childhood sexual victimization and sexual offending?

Responses to these questions were examined in the Research Findings Chapters; and are further discussed in this Chapter.

\section{Structure of the thesis}

Having introduced the research in Chapter 1, in Chapters 2 and 3 the focus was on the problem of CSA, as a means of placing in context the accounts of the men who participated in this study. In Chapter 2, the emphasis was on the prevalence of CSA, both 
in the general and offending populations; the characteristics of victims, and the factors which might render some children more vulnerable to CSA; the characteristics of perpetrators - including FSOs; and the issue of disclosure of CSA, and the silencing of victims.

Chapter 3 focused on the impact of CSA. The diverse nature of impact and variables associated with more negative consequences of CSA were explored at the outset. Then, attention shifted to: the concept of re-victimisation; impact and male victims; the extent to which some victims might report a positive impact; the impact of female-perpetrated CSA, and positive perceptions of that; and criminal behaviour as a consequence of CSA. Discussion of the latter was then extended to include sexual offending; and issues relating to the pathway from victim to victimiser were examined.

In Chapter 4, the emphasis was on Methodology: on the methods employed in undertaking the research; and the rationale for adopting those methods. First, issues relating to process were considered. Then, attention shifted to the particular challenges encountered in undertaking research with prisoners who had been convicted of CSA.

In Chapter 5, the theoretical underpinnings for the research were set out, in particular, the models/theories which were found to be of most relevance, and which informed analysis of specific aspects of the data set. In Chapters 6, 7, 8 and 9, the research findings relating to the 3 groups of offenders were presented: namely, those men reporting maleperpetrated CSA; female-perpetrated CSA; and male and female-perpetrated CSA. Each of the findings Chapters followed the same structure. First, their experiences of CSA were examined. This was followed by presentation of the findings relating to their perceptions of CSA, and its impact on them; and finally, their accounts of 'becoming an abuser'.

Offenders in all 3 groups share some demographic characteristics and it is not intended that all the data are revisited in this final Chapter. Rather, the key differences evident within and between groups are drawn out as a means of contributing to knowledge in this 
area; and informing policy and practice with both victims and offenders. In Part 1, the emphasis is upon 'understanding' the research findings as they relate to all 3 groups of offenders. In Part 2, a proposed 'model' of vulnerability is put forward, based on the findings. In Part 3, there is some reflection on the implications of the research for policy and practice with both victims and offenders. As has been the case throughout the thesis, distinctions are made between the following sub-samples in this Chapter:

- 32 men who reported experiencing CSA perpetrated by males

- 5 men who reported experiencing CSA perpetrated by females

- 3 men who reported experiencing CSA perpetrated by males and females

Moreover, difference is explored - as indicated in the research questions for the study on a number of levels, as noted below:

- Offenders' experiences of CSA

- Offenders' perceptions of CSA and its impact on them

- Disclosure

- Becoming an abuser

The Chapter concludes with some reflection on the contribution of the research to the existing knowledge base, and the limitations of the work.

\section{Part 1: Exploring Difference}

\section{Offenders' experiences of CSA}

Childhood is often portrayed as a time of innocence and play. In the writings of Rousseau (Aries 1986), children are characterised by innocence; purity, which should be respected and protected; natural goodness; and a sense of spirituality which places them closer to God and nature. Their innocence stems from a lack of knowledge and experience of the adult world, in particular, sexual activity; and a lack of evil or sin (Kehily and 
Montgomery 2003: 230). While many find such characteristics endearing, unfortunately, these are amongst the features so often cited by child sexual abusers as influential in drawing them to their victims.

The notion that: 'children are or should be sexually innocent is incorporated into ideas of childhood in general' (Ennew 1986: 11). Indeed, childhood has been defined as a 'golden age', in which happiness is associated with innocence (ibid.). All 40 men in this study had experienced contact CSA; their childhoods were far from innocent. Rather, they were characterised by sexual knowledge and experience inappropriate for their years; and that became their normality.

\section{Experiencing male-perpetrated CSA}

Offenders in this sub-sample share many characteristics with the men in the other 2 groups. However, there are a number of defining features which set them apart and these are drawn out here. Consistent with the research evidence, the majority (22) of men had been abused by those outside their own family or household; in 3 cases, by an unknown perpetrator. In several cases, offenders were abused 'in conjunction with other children' (Finkelhor 1984), both within care settings and external to those. In addition to sexual victimisation, the childhoods of many (20) of these men were also characterised by physical violence; and in 3 cases by domestic violence.

According to Boney-McCoy and Finkelhor (1995), the sexualisation of a child which occurs during CSA, may result in behaviour which places the child at further risk of abuse. Thirteen of the 32 men experienced re-victimisation in childhood. Some men experienced abuse within their own family first and then, also on an extra-familial level; others had been abused by more than one abuser who was known to them; some were abused by those in a position of trust, in particular, residential care staff; and also by other children and young people in care. Indeed, more than half (7) of the 13 men were re-victimised in residential care. 
Data on the age at which abuse began was not forthcoming from all the men in this subsample. However, it appears from the information provided, that many of these men were very young when first abused; and younger than the men in the other 2 groups. Indeed, 18 were 8 years or under at the onset of abuse. Almost a third (10) of the men in this subsample had been abused by those in a position of trust: including residential care staff; youth workers; a doctor; and another professional working in the CJS. Of note, is that 8 of the men were placed in residential care as children; and 7 of those were abused in that environment -4 solely by staff; 2 by staff and other children and young people in care; and 1 solely by his peers in care. A number of factors contribute to the concealment (Spiegel 2003) of abuse in institutional settings, including: the nature of institutions; and the power imbalance between staff and vulnerable children and young people, which aids abuse and ensures it remains well-hidden (Timmerman and Schreuder 2014). Moulden et al. (2010: 414) argue that professional perpetrators' relationships with their victims are marked out by: trust, authority, and 'limited disclosure'. For those men who were abused while in residential or care, their vulnerability and accessibility meant that the authority invested in care staff roles, both enabled abuse and served to silence their victims.

Just under a third (10) of the men had been abused by other children and young people; in 3 cases, by their brothers. Of interest, is that 6 of the 10 men who were abused by young males were re-victimised in childhood. Some research (Cyr et al. 2002: 963) indicates that the characteristics of sibling incest (brother-sister) do not differ from those of fatherdaughter incest and thus, the former should be recognised as 'a severe form of sexual abuse'. Increased attention should be paid to brother-brother abuse, given that evidence suggests if the trauma of sibling abuse is not addressed, victims may experience a range of negative consequences, including re-victimisation (Carlson et al. 2006). On that, it is interesting to note, that the 3 men who had been abused by their brothers, were also abused by those in a position of trust. 


\section{Experiencing female-perpetrated CSA}

Five men reported experiencing CSA perpetrated by females acting alone. None had spent time in institutional care; and so in that respect they differ from offenders in the other 2 groups. Two of the 5 men (Colin and Gareth) had also experienced physical violence in childhood. They had also both been abused by other children and young people. Of note, is that they were the most prolific sexual offenders in this sub-sample.

In contrast with those men abused solely by males - and with previous research on male victims - the majority (4) of the 5 men in this group had experienced intra-familial abuse. There are a number of points to note here. First, their abusers differed in age and also in their relationship with the men. In contrast with research which suggests that female perpetrators are more likely to be young women, Andrew was abused by his grandmother; and Bill by his mother. Two other men in this group were abused by other children and young people: Gareth by his sister; and Colin by a young aunt. Only one man - Eric - was abused by someone outside his family: a teacher. Second, the abuse the men experienced differed in form: only one man experienced penetrative sexual activity (Andrew). Third, having been abused by females in childhood, all 5 subsequently offended against female victims. This is consistent with Briggs' (1995) findings that males abused by females are more likely to abuse female victims.

None of the men in this group were re-victimised, while men in the other 2 groups were. It is possible that given the formers' positive perceptions of their abuse - discussed below

- they did not define the sexual activity as abusive or feel that they had been violated. Consequently, they did not experience the powerlessness (Finkelhor and Browne 1985) and helplessness (Summit 1983) evident in some of those abused by males; and their demeanour did not render them vulnerable to further abuse. While a contentious

proposition, it is possible, that in these cases, female-perpetrated CSA served as a 'protective’ factor. 


\section{Experiencing male and female-perpetrated CSA}

Three men reported experiencing CSA perpetrated by males and females. As was the case with the 5 men who reported abuse solely by females, all 3 men were abused by females acting independently of others; this was in addition to being abused by males. All 3 men had also been subjected to physical violence in childhood. Only one of these 3 men had been abused on an intra-familial level in childhood. Hugh, like Gareth, had experienced sibling abuse. Frank and David had experienced extra-familial abuse; their cases are distinguished by the fact that both had spent time in institutional care - an environment in which both had been sexually abused by males in a position of trust. Both these men had also experienced penetrative CSA perpetrated by one of their abusers: David's female abuser had engaged in full sexual intercourse with him; and Frank had experienced anal penetration perpetrated by his male abuser.

\section{Offenders' perceptions of the CSA experienced}

\section{Male perpetrated CSA}

The majority (14), of the 17 men who articulated their perceptions of this form of abuse clearly held negative views of that. Distinctions between the men's perceptions are drawn out next. These data shed some useful light on an area of enquiry which has to date received limited attention. The first thing to note, is that their narratives are characterized by traumatic sexualisation; betrayal; stigmatization; and powerlessness (Finkelhor and Browne 1985) to a greater extent than is evident in the narratives of the men in the other 2 groups. The latter was evident, for example, in their propensity to run away.

Those who had been abused on an intra-familial level reflected on the physical pain associated with abuse; on becoming fearful of adult males; and on the 'mental scars' resulting from abuse. The men who had been abused on an extra-familial level - many of whom had experienced CAP (Erooga et al. 2012) - recalled physical pain; an inability to trust; a sense of isolation; sexual problems; nightmares; an inability to keep friends; a 
tendency to engage in criminal behaviour; a fear of adults; confusion about sexuality; difficulties with relationships with females; shame; a lack of confidence; anger; disgust, repulsion; and a 'sense of feeling 'dirty' following abuse. Of interest, is that 2 men who held negative perceptions of their abuse (Oliver and Adam) recalled some sense of enjoying it. Their confusion was evident in their narratives: Oliver 'enjoyed' the abuse but also 'hated it sometimes', feeling 'ashamed', 'embarrassed' and 'dirty' as a result; and Adam recalled that he 'got pleasure' from the abuse in spite of the 'pain and disgust' he felt.

Fergus - who had been abused by a doctor - had mixed views of that experience; and referred to it as 'exciting but frightening'. Perhaps the non-intrusive nature of the abuse played some part in that perception. Interestingly, Bob was the only man to express positive views on his abuse. Bob had been re-victimised in childhood. He valued the attention he received from one of his abusers; and was prepared to tolerate the physical pain of abuse and being 'hurt' to achieve a sense of feeling 'special' that was lacking in his relationship with his father.

\section{Female perpetrated CSA}

Bourke et al. (2014: 769) posit that, in cases of FSO, the: 'dynamics between the victim and perpetrator are likely to be different to male perpetrated abuse'. This appears to hold true for the men in this sub-sample. Indeed, there is a distinct difference between these men's perceptions of abuse and the other 2 groups of offenders, in that all 5 viewed the abuse they had experienced positively. Evidence suggests that CSA characterized by a lack of force, where the victim is an adolescent, and the perpetrator an unrelated female, is less likely to result in victims' reporting negative consequences (Deering and Mellor 2011). One of these factors appears to feature here: none of the abuse involved force. However, only 2 females were adolescents; and in 4 out of 5 cases, the men were related to their abusers. There were no differences in perception according to the severity of the abuse. In turn, the men talked of 'enjoying' and 'delighting' in the sexual activity; perceiving it as 'natural' and 'normal'; and pleasurable. In contrast with those men 
abused by males, none of the men in this sub-sample reported negative consequences arising from their CSA: there was no evidence here, of the shame, fear, and pain so often expressed by the former.

\section{Male and female-perpetrated CSA}

Frank was the only man in this sub-sample to provide any indication as to how he viewed the abuse he experienced and its impact on him. Of interest, is the divergence in his views of the male-perpetrated as opposed to the female-perpetrated abuse he experienced. While he viewed the latter with a mixture of fear and excitement, his view of the former was wholly negative and his response was to run away. Of course, the nature of that abuse by a male was more intrusive, involving anal penetration; the female-perpetrated abuse he experienced involved exposure and masturbation. While this distinction in terms of the form of abuse experienced should, of course, be acknowledged, it should not be assumed that the impact of the latter is minimized.

\section{Disclosure}

\section{Male-perpetrated CSA}

The accounts of the men in this sub-sample provide some useful insights into the enduring problem of non-disclosure of CSA. While this problem persists, safeguarding and intervention can only be partially achieved; and will be restricted by the likelihood that most victims will not be reached by those agencies charged with detecting abusers and providing support to victims. Unsurprisingly, only 3 of the men in this sub-sample purposefully disclosed the abuse they experienced in childhood: each experienced a different form of abuse; each received an inappropriate response to telling; and all were silenced. An element of containment appears to characterize all 3 men's experience of telling. Each of those who heard those accounts of abuse, rather than acting on the disclosure, perhaps reflected on the potential loss - whether personal or organizational which might be incurred by responding appropriately. It is possible that such responses 
resulted in the reinforcement of feelings of helplessness and betrayal so often demonstrated by the men in this sub-sample.

Five other men told no-one of their abuse until they were imprisoned. Amongst the explanations put forward for this were: a reluctance to relinquish the 'privileges' received as a result of abuse; fear; threats from the abuser; not acknowledging abuse 'as an issue'; a lack of sexual knowledge; a mother's inability to protect, and fear of a father's violent disposition; consistent messages from abusers that victims would not be believed; and abuse framed as a punishment for bad behaviour.

\section{Female-perpetrated CSA}

Victims abused by females are less likely to disclose than those abused by males. This situation is compounded where victims are male. If the perpetrator is female, societal beliefs about the caring and nurturing aspects of the female role; and perceptions that males are more likely to perpetrate abuse while females are more likely to be victims, predominate, and inhibit disclosure (Denov 2003; Denov 2004). Unsurprisingly, therefore, only one of the 5 men in this group - Andrew - attempted to tell of the abuse perpetrated by his grandmother. Perhaps fear drove his decision to make a purposeful (Sgroi 1982) disclosure. Whatever the origin of that decision, and in spite of the fact that Andrew told both parents independently, unfortunately, their response was inadequate and inappropriate. Indeed, the abuse continued unchecked and escalated in severity, from touching under clothing to penetrative sexual activity which persisted over several years. Two other men explained why they had not previously disclosed their abuse: Bill was fiercely loyal towards his mother and made a decision not to tell of her abuse, which lasted for many years, based on fear of the consequences. Gareth's sense of isolation appeared to be a contributory factor in inhibiting disclosure - he felt that 'he wasn't close enough to anyone' to tell of the abuse perpetrated by his sister.

Male and female-perpetrated CSA 
Only one man in this sub-sample (Frank) disclosed abuse in childhood: interestingly, the male-perpetrated, as opposed to the female-perpetrated CSA he experienced. As has been evident in the other 2 groups of offenders, in the few cases where purposeful (Sgroi 1982) disclosure was made, the response was inappropriate and inadequate. In Frank's case, the response he received from care staff - and also from his peers - left him feeling humiliated.

\section{Becoming an abuser}

Male-perpetrated CSA

There are a number of features which distinguish the men in this sub-sample from the other 2 groups of offenders. The majority (24) had previous convictions. Just under a third (10), had previous convictions for sexually offending against children; 6 of those had served a prison term for those offences. Three of those 10 men had also committed a range of other non-sexual offences. Therefore, based on previous convictions, 7 could be defined as specialist (Harris et al. 2009) offenders (Bob, Edward, John, Mark, Oliver, Donald and Carl). Interestingly, these are the same 7 men who reported that they became aware of a sexual interest in children before the age of 16. All except Bob and Edward had served prison sentences for sexual offences against children.

Unlike the men in the other 2 groups, a small number of offenders (2) in this sub-sample had been convicted of offences involving IIOC accessed through the Internet; one of those - Gavin - was convicted of possessing and distributing those images and was the only man of the 40 to have engaged in non-contact CSA. (The other man also committed contact CSA). It is worth noting that - given the recent increase in Internet offending had the research been undertaken in 2016, it is likely that a larger number of offenders might have presented with this offending profile.

Adolescents who abuse their siblings are more likely to have been part of a dysfunctional family and to have been sexually abused, than those who offend against other children 
and young people outside their family (Worling 1995). The former are also more likely to have been exposed to domestic violence within their family. Donald appears to fit this profile. He had been physically and sexually abused by his father from the age of 5 . He abused his sister at the age of 12; and then abused unknown victims before progressing to abuse his own sons. Young people who engage in sexually harmful behaviour (SHB) are not likely to 'specialize' in sexual offending or to perpetrate sexual abuse as adults (Caldwell 2007: 108). Rather, they are more likely to be subsequently convicted of nonsexual offences (Hackett 2014). This does not appear to be true of Donald. Neither does it appear to be true of Gareth, or John, both of whom began sexually offending in adolescence. Gareth was abused by his sister at the age of 11 . When that abuse ended, he abused his niece and a further 11 victims. John had first been sexually abused by his brother at the age of 6; then by a member of staff in residential care; and also an 'expupil' at the home. John's family life was highly sexualised; and he abused one of his brothers and his sister. He was the only man of 40 to have offended with a co-perpetrator. All 3 of these men experienced physical violence in childhood; and each had a lengthy history of sexual offending. Clearly, all presented a considerable risk to children.

There appears to be some consistency in Gavin's account with the findings of Glasser et al. (2001: 492) that: 'the abuser's target age-group is usually limited to the age when he was himself abused.' Gavin had been abused by a pupil at his school and explained that his attraction to children was: 'invariably to boys the same age as I was when I was being abused'. Clive's offending appears to be a 're-enactment' (Howitt 1995: 2) of the abuse he experienced: '[I did] the same as what was done to me, masturbation and things like that'.

Some men identified factors which, in their view, contributed to their offending. These included: childhood isolation; the normalising of CSA; a need to care for someone; a lack of 'love' in childhood; and the need to counteract the sense of powerlessness experienced as a victim. With regard to the presence of Core ITs (Ward and Keenan 1999) in their narratives, in 17 (out of 23) cases, there was evidence of the IT 'Children as Sexual Objects'. Fifteen of the men referred to a sense of 'Uncontrollability' in their offending; 
and 11 clearly viewed the world as a 'dangerous' place. Four men's narratives were characterized by a sense of 'Entitlement'. Three of those men - Robert, William and Donald - had abused several of their own children. Parental control and power appear to have featured for these men, alongside the availability and accessibility of their victims. Oliver, on the other hand - who had a history of offending against unknown male victims - was of the view that having been forced by his abusers to comply with their demands, he was entitled to take the same approach with his victims and so: 'taught them to feel that they had no choice'. Of interest, is that a sense of 'Entitlement' was not evident in the accounts of any of the men in the other 2 groups.

\section{Female-perpetrated CSA}

Three men in this group had previous convictions; one of those (Colin) had previously been imprisoned for sexual offences against 4 female child victims. Colin appears to have specialized (Harris et al. 2009) in sexual offending against children over many years. Three men had abused 1 victim; and one man, 2 victims. However, Gareth had abused 12 children over a 12 year period; and crossed relationship and age boundaries in terms of his offending. Interestingly, as already noted, he had been abused by his sister in childhood; and was one of 2 men in this group to have been abused by other children and young people (the other was Colin, who had been abused by a young aunt).

None of the men in this sub-sample reported accessing IIOC or using the Internet to pursue a sexual interest in children. Two men (Andrew and Bill) recognized that they replicated aspects of their own abuse when offending against their victims. For Bill, a number of factors combined to result in his sexual offending: namely, the abuse he experienced; the normalisation of an abusive environment at boarding school; a lack of stability in his family life; and his subsequent promiscuous adult lifestyle.

There were distinctions in terms of the presence of Core ITs (Ward and Keenan 1999) in these men’s narratives. Clearly, all 5 perceived 'Children as Sexual Objects’ (ibid.). Moreover, a sense of 'Uncontrollability' was evident in 4 of the men's accounts. 
According to Andrew, the abuse perpetrated by his grandmother led to his offending. For Bill, a lifestyle characterized by alcohol and sex fuelled offending. In Eric's case, 'situational stressors' (Finkelhor 1984) including ill-health and sexual difficulties featured. For Gareth, abuse became an ‘addiction’: he ‘just couldn’t stop doing it’.

The IT 'Dangerous World' was evident in 3 men's narratives (those of Bill, Gareth and Colin). Each referred to children as meeting an emotional need. However, there are also distinctions here: while Bill was attracted to the unassuming and accepting nature of children, he also had the capacity to engage in relationships with adults. Gareth and Colin on the other hand, found 'safety' only in children's affection and innocence. Interestingly, the IT 'nature of harm' was present only in the narratives of Gareth and Colin. Both minimized their offending; insisting that their abuse caused no harm; and in Gareth's case, that it was perpetrated in a 'safe' environment. 'Entitlement' did not feature in these men's narratives. Perhaps the fact that none of the men abused as fathers played a part in this. Although Andrew, of course, did abuse his stepdaughters. Alternatively, their experience of female-perpetrated CSA and their positive perceptions of that might prove relevant.

\section{Male and female-perpetrated CSA}

All 3 men in this sub-sample had previous convictions. Hugh and Frank had previously been convicted of sexual offences against adult females: namely, rape; and indecent assault. They had each served a prison sentence for those offences. However, they also had convictions for a range of other non-sexual offences. In contrast with the other 2 groups of offenders, there is some consistency here with regard to the men's relationship to their victims. All 3 men offended against children who were biologically related to them. David abused his daughter; Frank, both his daughter and granddaughter; and Hugh his son and daughter. In contrast to the research evidence, David and Frank, having been abused by both males and females, later perpetrated abuse against female victims. However, Hugh crossed gender boundaries, abusing both his son and daughter. In David and Hugh's case, the abuse was penetrative in nature. As was the case with the men who 
had been abused solely by females, none of the men in this sub-sample reported accessing IIOC, or using the Internet to pursue a sexual interest in children.

There are some parallels with, and some distinctions between, these men and the other 2 groups of offenders, in terms of the presence of Core ITs (Ward and Keenan 1999) in their narratives. Clearly, all 3 perceived ‘Children as Sexual Objects’ (ibid.). All 3 also referred to a sense of 'Uncontrollability' resulting from difficulties or stressors which, in their view, served as precursors to offending: namely, relationship and financial difficulties. Interestingly, none of the men referred to their own abuse as a contributory factor in their offending; and none of the other 3 Core ITs were evident in the men's accounts. Of course, given that these men were more reluctant to discuss their own abuse and their offending behavior than the men in the other 2 groups, a cautious approach is needed in drawing any conclusions from these findings.

Next, in Part 2 of this Chapter, the focus shifts to the factors inherent in vulnerability to become a victim and also a perpetrator of CSA, as evidenced in the men's narratives. 


\section{Part 2: Developing a 'Model' of Vulnerability}

Ward et al. (2006: 30) note that: 'most offenders are defensive about what they have done and reluctant to talk about it in any detail', however, this was not borne out in this study. Indeed, given the time and space to reflect on their childhood and offending, many offenders did talk about both aspects of their lives. The proposed 'model' of vulnerability presented in this section of the thesis is based on the men's accounts; and on what can be gleaned from those about the factors that might render some children more vulnerable to CSA; and some victims more vulnerable to becoming an abuser. Of course, this 'model' has its limitations. The sample is small; it represents a 'snapshot' of a particular population, at a particular point in time; it comprises a population which is likely to have experienced - and perpetrated - more severe forms of abuse; and thus, the findings cannot be generalized. Its strength, however, lies in its construction around an alternative data source: child sexual abusers’ narratives.

Spiegel (2003:19) posits that:

'The ultimate goal of a model is select, organize, and present data within a ...comprehensive framework in order to consider in a meaningful way data that might otherwise remain disparate and of limited utility. Moreover, a model reflecting human behavior in the social environment must validate and authenticate the realities as experienced by individuals, families and groups. In other words, a model must tell their stories, tell their truths.'

In this study, the aim in constructing the proposed 'Model' was to: further understanding of offenders' perspectives on their CSA; to present those perspectives in a way which would be meaningful to policy and practice in the field; and above all to: 'tell their stories, tell their truths'.

As already noted, the 'Model' comprises 2 parts and the factors associated with each, are evidenced in the men's narratives. First, the existing evidence on vulnerability to become a victim is explored; and parallels are drawn between existing research evidence and that collected for this study. 


\section{Becoming a victim of CSA: vulnerability}

There are a number of factors which render children more vulnerable to CSA: these were discussed in Chapter 2 and are briefly revisited here. Notwithstanding the power imbalance between adults and children, some children are even more vulnerable to CSA than others, for example, children with disabilities (Miller and Brown 2014). Moreover, children who have previously experienced physical, sexual, or emotional abuse (Berliner and Conte 1990; Fleming et al. 1997); or a lack of 'physical affection' from a father, which results in an inability to detect 'genuine affectional interest on the part of an adult' as opposed to sexual interest (Finkelhor 1979; 1984: 26); are isolated from their peers (Elliott et al. 1995); live in a household characterised by domestic violence; and whose parents misuse substances are also more susceptible to abuse (Goodyear-Brown 2012). Each of these factors are of relevance, to differing degrees, to the 40 men who participated in this research.

In addition to sexual victimisation, the childhoods of 25 of the 40 men were also characterised by physical violence ( 20 of those had been abused by males; 2 by females and 3 by both males and females). This is unsurprising, given that where there is physical violence in the family, it is more likely that CSA is also perpetrated (Cawson 2000). However, while in some cases, physical violence accompanied the CSA the men experienced - particularly for those who were abused in institutional settings - in other cases it was meted out by those who did not abuse them sexually. Some men lived in environments characterised by domestic violence; and others recalled the sense of isolation they experienced in childhood. Poor relationships with fathers also featured for some men. This appeared to render them more vulnerable to become 'preferred targets' for abusers; and emotionally dependent on the abusive relationship (Wallis 1995:11). There is evidence of that dependence in several of the men's narratives.

Some of the 32 men in this sample who had been abused by males, described how they identified children with specific characteristics who would be vulnerable to abuse; and in some cases, they drew on their own victimisation to assist with that. In Figure 10.1, the 
factors which appear to have rendered both offenders' and their victims vulnerable to CSA have been teased out from the men's narratives. 
Figure 10.1: Factors associated with vulnerability to become a victim of CSA - as evident in a sample of 40 adult male prisoners convicted of child sexual abuse

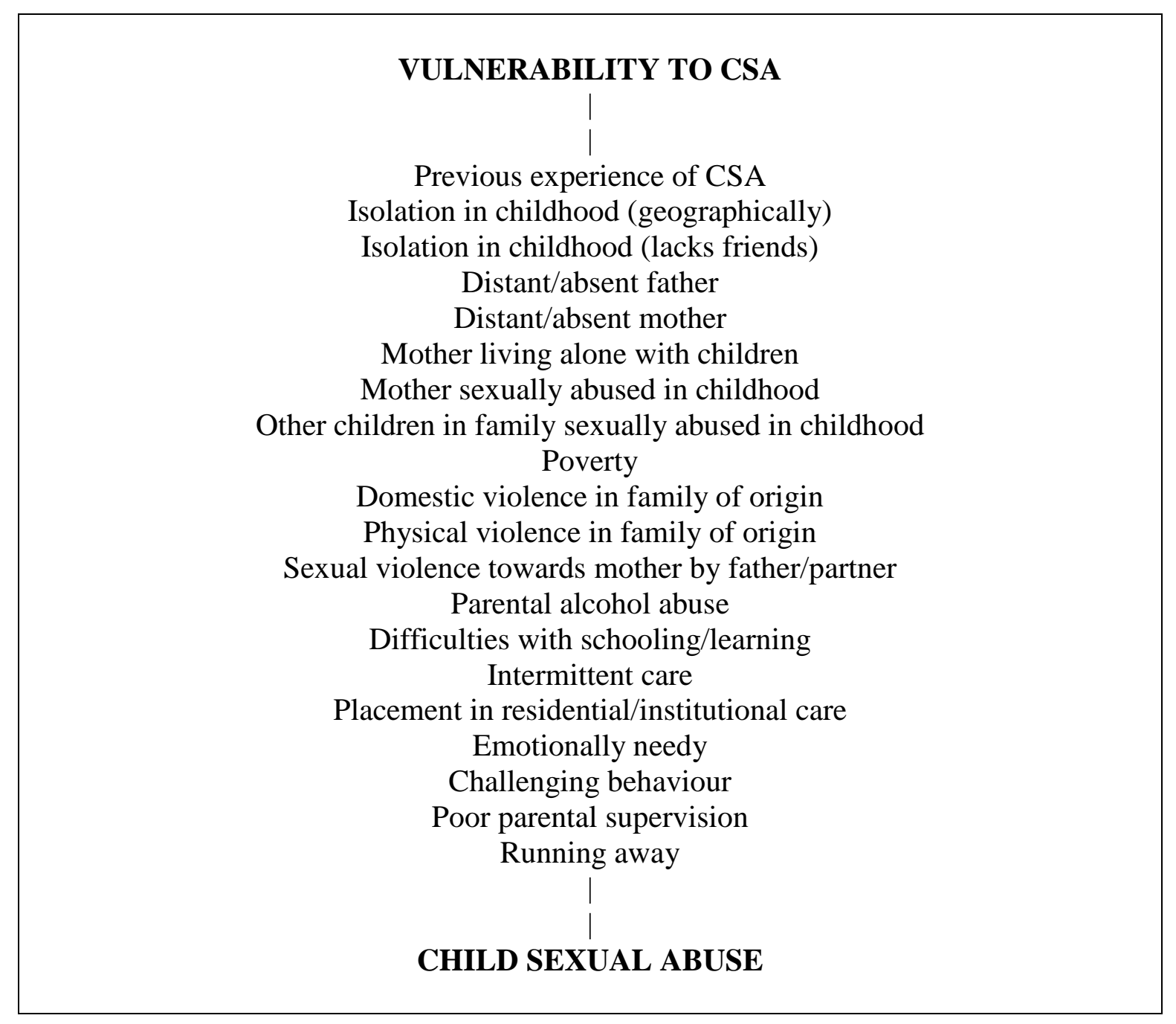

Each of the factors listed above featured in offenders' narratives to some extent, both in relation to their own childhood and in their accounts of how they detected vulnerability in their victims. They were not all present in all the men's accounts; however, some, for example, physical violence, were more evident than others. The process of victimization is complex and multi-factorial in nature. It is not likely that one factor alone will render a child more vulnerable to CSA. Rather, it is likely that any number of the factors listed above, may combine to increase vulnerability. This appears to have been the case for the men in this sample. All 40 of the men lived through childhoods which were characterised by sexual violence, and sexual activity which was inappropriate for their stage of development. Some of the men (10) were placed in residential/institutional care; this was 
an environment in which they were unsafe, and in which the majority (9), were sexually abused. These men were already vulnerable when placed in that setting. Some experienced dysfunctional family environments where both sexual and physical violence were the norm; they therefore presented as ready victims for those in positions of formal power in institutional environments. Those in a position to care and protect have often used the power invested in their roles to intimidate and create an environment characterised by fear (Timmerman and Schreuder 2014) where abuse can flourish. The presence of such an environment; and a corresponding sense of fear came through clearly in several of the men's narratives.

Thirteen men were abused by other children and young people, both male and female: in 5 cases by their siblings. Moreover, 13 men were abused by those in a position of trust, in particular residential care workers. Also of note, is that 16 men experienced revictimisation in childhood; 25 experienced physical violence; and for 3 others, domestic violence also featured in their childhoods.

Some offenders had difficulties with schooling and learning; others were socially isolated from their peers, or isolated in a geographical sense. Yet others appeared emotionally needy. Each of these factors exacerbated the men's vulnerability to CSA. Of interest, is the extent to which they recognized - and exploited - that vulnerability in their child victims. Having themselves demonstrated vulnerability as children, the men sought out victims who also did so. Therefore, their tendency - as evidenced in their narratives was to identify those: who were 'lonely and 'in need of attention'; without a father or other male living in the household; with 'emotional problems' or challenging behaviour; who were living in poverty and 'had nothing'; and those who appeared less well supervised than other children, in the sense that 'they wander about the streets'. Also of importance to some of the men, was the child's reaction to them, or the sense in which they are 'open' to abuse. Edward, for example, sensed that one of his victims had been previously sexually abused because of the child's response to physical contact: that response made him 'very susceptible to being abused again'. 
For Oliver - who had been abused in residential care - an environmental awareness aided his offending; in particular, his knowledge of where children running away from care would gravitate towards: 'because he had been there himself'. Dan recognized that his victim's mother and family were vulnerable due to their previous experiences of CSA. That assisted with his offending; and in his view served to inhibit his victim's disclosure. Offenders are adept at identifying vulnerability and seek out opportunities to offend in whatever form those present. The challenge for those involved in safeguarding lies in identifying and understanding the factors which increase vulnerability; and which are so individual to a child and their environment. Offenders appear to have developed this understanding and thus, are provided with ample opportunity to abuse.

In Figure 10.2, the emphasis shifts to the factors which appear to have played a part in becoming a child sexual abuser. Again, these factors are particular to the 40 men in this research, and have been drawn out from their narratives. 
Figure 10.2: Factors associated with becoming a perpetrator of CSA - as evident in a sample of 40 adult male prisoners convicted of child sexual abuse

\author{
CHILD SEXUAL ABUSE \\ $\underline{\text { \& }}$
}

Environmental components:

Physical violence in family of origin, including by mother

Domestic/sexual violence in family of origin

Isolation

Poor relationship with caregivers

Institutional/residential care

Characteristics of CSA:

Use of force

'Painful' nature of abuse

Exposure to pornography

Re-victimisation

Multiple perpetrators

Abuse by those in position of trust

Abuse by children and young people

Abuse by females

Responses - offenders:

Perceptions of abuse

Betrayal: including mothers' failure to protect/respond

Powerlessness

Helplessness

Running away

Abandonment: no intervention/protection; rejection by abuser

Humiliation

Shame/Stigmatization

Normalisation of abuse

Unresolved anger at abuse

Accommodation: accepts pain of abuse as:

a means of survival

Tolerates abuse as:

a means of gaining care and attention

Fear of adults

Inability to cope with/understand own abuse

Responses - others:

Collusion to conceal abuse

Inappropriate response to disclosure - minimization/denial 
Most CSA victims do not subsequently sexually offend. Evidence suggests that there is an association between experiencing CSA and later sexual offending against children (Nunes et al. 2013), however, the problem lies in determining which factors prove more influential in this. As noted in Chapter 3, a number of factors have been identified in previous research on the 'victim to offender cycle' (Thomas and Fremouw 2009: 386). These include: a childhood marked out by sexualisation: namely, sexual contact with adults, peers and siblings; 'disturbed' family background, particularly in terms of relationships between father and son; and running away (Langevin et al. 1989: 228); emotional abuse and/or physical neglect (Craissati et al. 2002); experiencing physical violence in childhood (Widom and Ames 1994); poor maternal care and/or an absent parent; and experiencing female-perpetrated CSA (Briggs and Hawkins 1996; Glasser et al. 2001). Others have pointed to a childhood characterised by 'heightened sexuality': namely, exposure to pornography at an early age (Simons et al. 2002; 2008); sexual activity with animals; insecure parental attachment (Simons et al. 2008); and multiple episodes of abuse by more than one abuser (Connolly and Woollons 2008).

Similar results have been found in the population of children and young people who have engaged in SHB. Here, the following feature: witnessing and experiencing physical violence, and 'discontinuity of care' in childhood (Bentovim and Williams 1998: 104); more forceful forms of CSA, involving penetration (Veneziano et al. 2000); abuse by both males and females (Burton et al. 2002); neglect, family violence, poor parental supervision and female-perpetrated abuse (Salter et al. 2003); family history, parents' characteristics, an unsupportive response to disclosure; and a sense of shame, anger, anxiety and confusion post-abuse (Johnson 1989).

Consistent with previous research, in the data collected for this study there is evidence that a number of factors might have contributed to the men's sexual offending, in addition to the CSA they experienced. Of course, given that this research is small-scale and qualitative in nature, it is not possible to 'measure' in statistical terms the strength of each factor; and the association between each. Such limitations are fully acknowledged; 
and these data are put forward tentatively, based on their capacity to offer an alternative individual and therefore more meaningful - source of information on this topic.

Each of the factors listed in Figure 10.2 featured in the men's narratives to some extent, in addition to CSA. Some of those factors relate to the environmental components of their childhood; some to the characteristics of the abuse they experienced; and some, to their and others' response to abuse. These are explored below.

\section{Environmental components}

As was the case in Figure 10.1, some factors were evident in the men's lives more than others. As already noted, the men's childhoods were often highly sexualized and violent. Some experienced isolation in a geographical sense; and also from their peers and other family members. Moreover, some of the men cited poor relationships with caregivers; and 10 of the 40 men were placed away from home in institutional/residential care, in some cases for many years. Taken together, one sees evidence of a childhood environment which was dysfunctional on a number of levels, as opposed to nurturing.

\section{Characteristics of the abuse}

There is evidence of diversity in the men's accounts, with regard to the characteristics of the abuse experienced. Several of the men abused by males experienced CAP and reflected on the use of force during abuse and its 'painful' nature, for example. While only a small number of men were exposed to pornography as part of their abuse, of particular note, is the number who experienced: re-victimisation; and abuse by multiple perpetrators, those in positions of trust, and children and young people. The impact of each of these distinct abusive experiences warrants further examination. 


\section{Offenders' responses}

Offenders' responses to their abuse differed. While female-perpetrated CSA was viewed positively; the majority of men who were abused by males held negative views of that. As was the case in Mendel's (1995) research - albeit with males who were victims of CSA but who did not later perpetrate abuse - there was evidence of betrayal, traumatic sexualisation, powerlessness and stigmatization (Finkelhor and Browne 1985). Some men's accounts were characterized by a sense of betrayal by those in a position to protect them, or to respond to their disclosure. For others, powerlessness and helplessness featured, as they recognized the 'power' of their abuser; and that they had no option but to submit. In these cases, the men's response was often to run away. A sense of abandonment came through in some of the men's narratives: this was characterized on the one hand by the understanding that there would be no intervention or protection for them; and on the other, by the knowledge that they were rejected by an abuser who lost interest in them. Taken together, this might have contributed to a sense of isolation and stigmatization (Finkelhor and Browne 1985).

While others experienced humiliation and shame resulting from their abuse, some recognized that being part of a pervasive abusive environment resulted in normalization of their abuse. In some cases, there was evidence of unresolved anger; other men recalled tolerating or accepting the 'pain' of abuse as: a means of survival; and of gaining care and attention which was otherwise lacking in their lives. For some, an enduring effect of their abuse was a fear of adults. Several of those who had been abused by males expressed concern that they had been unable to deal with, or understand their own abuse; that, they felt, presented a barrier to desistence. Of note, is Craissati et al.'s (2002: 236) observation on the significance of 'unresolved childhood sexual victimisation', which, when combined with other negative childhood experiences, may result in emotional problems and also difficulties with relationships in adulthood. In such cases, individuals may be at risk of becoming abusers themselves - and in particular of abusing male victims - in their efforts to 'resolve early sexual trauma' (ibid.: 236). 


\section{Others' responses}

Bourke et al. (2014: 777) found 'no differences' between the disclosure rates of victims abused by females or males: the majority of victims in both groups did not tell anyone of the abuse. The majority of men in this sample, too, did not disclose the abuse they experienced. In total, 5 out of 40 men disclosed in childhood, however, in each case, the response they received was inadequate. Others chose not to report the abuse. Spiegel (2003: 100) notes that males are less likely to disclose abuse; to be protected by their family if they do disclose; or encounter 'parental concern' about the impact of abuse, aside from anxieties about sexual orientation (ibid.). This observation appears to hold true for the men in this research.

Children invest much in making their decision to tell; the repercussions of such responses should not be under-estimated, given that a hostile or non-supportive response can impact on 'recovery' (Fontes and Plummer 2010: 491; Easton 2013). In this research, it appears that it might not have been inappropriate responses to disclosure alone which had a negative impact on the men. Rather, the message transmitted in those responses might have been more impactful: namely, that female-perpetrated abuse was minimized and denoted as harmless and merely a demonstration of 'love'; and that a family's financial security, and family and organisational reputations were of greater value than the protection of a child victim. When CSA is detected, it is crucial that the response of parents/carers, professionals and others in a position to protect, is appropriate and supportive of the child victim. For the men in this research, it is possible that the sense of further betrayal they experienced at the point of telling - and perhaps a sense of their own worthlessness - from those in a position to protect, compounded the negative consequences of CSA. 


\section{Part 3: Implications of the research}

In this thesis, the emphasis has been on exploring difference within and between groups of offenders; and on the 'meaning' of childhood sexual victimization to those later convicted of - and imprisoned for - CSA. Here, the focus is on the implications of this research for policy and practice with both victims and offenders.

\section{Implications for policy and practice: future directions}

In the midst of much contradiction, there is some agreement that CSA is a global problem which affects 'millions of children' (Stoltenburgh et al. 2011: 90); and which has the potential to devastate victims' lives (Saied-Tessier 2014). The emphasis often, however, is on CSA as a gender-based offence, with males as perpetrators and females as victims. This is borne out in recent policy initiatives in the UK, including, for example, the Violence against Women and Girls initiative (CPS 2015).

In the face of evidence which suggests that females are more likely to be victims of CSA, males who have experienced such violence are a: 'stigmatized, under-studied, and marginalized population' (Easton 2013: 344). Indeed, it appears that a 'sociocultural disregard for sexually abused boys' (Spiegel 2003: 175) persists. This is based on assumptions, or myths that: boys are not sexually abused; if they are, it happened because it was something they wanted; if abused by a male, the victim must be gay; if abused by a female, it was not really abuse; and boys who are sexually abused are not able to take care of themselves (Spiegel 1995). Such myths inhibit understanding and also disclosure. Clearly, there is a need for attitudinal change on a number of levels. The starting point should be some recognition that CSA is not gender (or age)-based; that females can be perpetrators; and males victims; and that children and young people engage in SHB in significant numbers. Indeed, it is only through acknowledging and examining 'all types of child sexual abuse that we may increase our awareness and understanding' of this complex phenomenon (Denov 2004: 198). 
In the narratives of the 40 men who participated in this research, there is evidence of heterogeneity in the CSA they experienced; and that which they perpetrated. The characteristics of the CSA they experienced took varying forms; and the gender, age, and status of their perpetrators differed. There were variations in: their perceptions of that abuse, and its impact on them; their offending; and also the belief systems which underscored that offending. Yet, little attention is paid to such distinctions in work with child sexual abusers, where the emphasis is on a collective, as opposed to individual approach to intervention (Colton et al. 2009); and the grouping of perpetrators together, irrespective of their victimization experiences (Glasser et al. 2001). The findings of this research suggest that questions should be posed as to the efficacy of such an approach.

Much preventative emphasis has been placed on alerting children and young people to the potential for abuse to be perpetrated; this approach can form 'only part of a broad solution' (Whitaker et al 2008: 530), however. The under-reporting of CSA is a significant problem; and the detection of CSA heavily reliant on victims' disclosures. However, the 'burden of responsibility' (CCE 2015: 13) should not be placed on victims. Rather, preventive efforts should be widened to focus on the offender's behaviour and an understanding of: 'the factors that lead to the development of the initial perpetration of child sex offending' (Whitaker et al. 2008: 530). The emphasis should be upon increasing understanding of the diverse nature of CSA: those who perpetrate it and experience it; and the complexity of the disclosure process, in particular, victims' need for: 'a supportive structure or scaffold in order to reveal their experiences' (Jensen et al. 2005: 1396). Moreover, the extent to which families may try to 'manage' CSA allegations warrants attention, as those attempts will usually involve the 'silencing' of victims (CCE 2015: 13); and are often grounded in efforts to avoid collective shame and humiliation (Gilligan and Akhtar 2006), and to protect reputation, whatever the cultural setting of CSA.

The criminal justice environment is characterised by community protection (Ward 2014: 130); and policy and practice with offenders is grounded in the identification and management of risk, and 'risk-centric discourses' (McAlinden 2014: 180); and a reactive 
approach to the problem of sexual offending against children. Of course, it is crucial that there is an emphasis on understanding risk and reducing levels of future victimization. Perhaps we can learn from abusers themselves as to how this might be achieved. For, to truly understand risk, we need to explore the individual nature of CSA, and its dynamics and effects. This would require a move away from the existing 'preoccupation with measurement' towards an alternative qualitative approach; and a focus on individual experience and beliefs (Ward 2014: 140). Acknowledging and understanding difference within and between offender groups appears to be a useful starting point. Indeed, a more individualized and targeted - as opposed to a one-size fits all - approach (Colton et al. 2009), which enables offenders to understand their own victimization and the extent to which it contributed to their offending might facilitate desistence; mitigate risk; and importantly, allow offenders the opportunity to live better lives on release (Ward 2014).

\section{Conclusion}

In this small-scale, qualitative, descriptive study, the focus has been on 40 adult males who had been convicted of - and imprisoned for - CSA, and their accounts of their childhood sexual victimization; their perceptions of that and its impact on them; and the extent to which, in their view, it might have contributed to their later sexual offending. Thirty two of those men had been abused by males; 5 by females; and 3 by males and females. The approach adopted throughout this thesis has been one of exploring difference within and between these 3 groups of offenders.

The men's accounts shed some light on CSA from a perspective not often sought, or heard: on the concept of victimisation and vulnerability; and the factors associated with becoming an abuser, as understood by abusers themselves. As is the case in Durham's (2003: 311) research the emphasis here has been on: 'diversity and the uniqueness of individual experiences.’ During the course of the research, as with Durham:

'a commonality of experience was identified across this uniqueness. This did not deny or compromise the uniqueness of each... experience, but rather served to highlight common struggles, concerns and fears' (ibid.). 
Even within this small sample, it is clear that although the men share some characteristics; and there is 'a commonality of experience' (ibid.); they also differ on a number of levels. Of note, is that there are differences within groups that extend beyond the gender of those who abused them in childhood, these relate to: the form of abuse experienced - intra or extra-familial; the age of the perpetrator - with several men being abused by children and young people; the status of their abuser - several were abused by those in positions of trust, in particular while in institutional/residential care; and the extent to which they experienced re-victimisation. Further distinctions are evident with regard to perceptions and the impact of the CSA; and the men's accounts of becoming an abuser. Evidence of such diversity within such a small group of child sexual abusers provides justification for the approach adopted in this thesis; and reinforces the need for a more individualized approach to intervention with child sexual abusers.

\section{Outcome of the research: contribution to the knowledge base}

This research sheds light on the dynamics and effects of CSA as articulated by a particular population: prisoners who had been convicted of CSA. The men's narratives expand the already existing evidence base, in particular, in relation to: the sexual victimization of males; male re-victimisation in childhood; female-perpetrated CSA; SHB by children and young people; CSA perpetrated by those in a position of trust; residential/institutional CSA - including abuse by staff and also by peers in care; and disclosure of CSA.

\section{Limitations of the research}

Of course, the research is limited by the fact that the sample is small and unrepresentative; and this has already been acknowledged. Moreover, the 40 offenders upon whom this work is based are distinguished by the fact that they have been caught and convicted of sexual offences against children. Those who remain undetected may well present with different characteristics (Glasser et al. 2001). The work is also limited by the fact that while some men were prepared to discuss their childhood and offending 
in detail, others were more reluctant to do so. However, despite its limitations, the research is of value due to its focus on the stories (Plummer 1995) and perceptions of offenders themselves: a viewpoint often missing from the research evidence. It appears there is an opportunity to learn much from prisoners convicted of CSA about sexual victimisation and offending. To ignore such a data source would be foolhardy, given the gaps in our understanding of CSA. Indeed, as Dodsworth (2014: 189) notes of her participants (women involved in sex work in the UK) whose stories, like those of sexual offenders, are not often heard: 'They are experts in their own lives and should play a key role in informing policy and service interventions'. Notwithstanding their limitations, it is hoped that the findings reported here, will at least initiate some further debate on the issues which have been raised; and which are so pertinent to many of those who have experienced CSA; and who have perpetrated sexual violence against children.

\section{Chapter summary and final thoughts}

In Part 1 of this Chapter, the emphasis was on exploring difference within and between the 3 groups of offenders who participated in this research. Clearly, the men share some characteristics but they also differ with regard to their experiences of CSA; their perceptions of that; its impact on them; and the belief systems which underscored their offending. They differ, too, in that only a small number of men could be defined as specialists (Harris et al. 2009), with over half (17) committing a range of other offences. One of the defining characteristics of the sample was pervasive secrecy (Summit 1983; Spiegel 2003). This was evident on two levels: in the sense that few men disclosed abuse in childhood; and that some remained reluctant to talk about their childhood sexual victimization as adults.

It appears that victimisation may be one of several factors which together contribute to sexual offending. Some (Epps and Fisher 2004) suggest that there are a number of psychological processes which might explain the part played by victimization. Offenders, for example: may re-enact their own abuse; and may have learnt - as part of an abusive environment - to abuse. Here, the concepts of modelling and social learning feature. 
They may try to acquire power and control through becoming an aggressor, in an attempt to deal with earlier negative and traumatic experiences; and may experience sexual arousal through fantasising about their own abuse - here, conditioning features.

It appears that each of these processes played some part in some of the men's offending. Of note, however, is the individual nature of their victimization and their response to it. A small number of men, for example, replicated their own CSA, and drew on fantasy as part of their offending. Moreover, the narratives of those abused by males were more likely to be characterized by a learnt response; and the acquisition of power and control.

In Part 2, attention shifted to a proposed 'model' of vulnerability, based on the men's accounts; and on what could be gleaned from those about the factors that might render some children more vulnerable to CSA, and some victims more vulnerable to becoming an abuser. A range of factors were evident in offenders' narratives to varying degrees; and these are summarized in the 'model'. The men's accounts shed some light on the complex, multi-factorial nature of victimization and subsequent offending. In that sense, they are of value to those seeking to provide more effective treatment and intervention with both victims and offenders.

In Part 3, the implications of the research for policy and practice - and future directions were considered. Attention was drawn to the need for recognition that CSA is not gender (or age)-based; that females can be perpetrators; and males victims; and that children and young people engage in SHB in significant numbers. Against a background of criminal justice policy and practice grounded in the identification and management of risk, a reactive approach to the problem of sexual offending against children prevails. In mitigating risk, and working towards prevention, the value of a more individually based approach to intervention with offenders; and the centrality of the disclosure process for victims was highlighted.

Finally, in bringing this Chapter to a conclusion, there was some discussion around the contribution of the research to the existing knowledge base; and the limitations of the 
work. The extent to which there are links between experiencing CSA and becoming a perpetrator has been the focus of much research for some time now. Yet, the evidence is conflicting and a plethora of 'unanswered questions’ persist (Craissati et al. 2002: 225). In the midst of much research activity, little attention has been paid to the perspectives of prisoners convicted of CSA, and the sense of difference noted in this thesis. If we are to provide the optimum levels of protection for children, and implement more effective intervention with offenders, it is crucial that we explore those differences where they exist in order that we understand more fully: 'the relationship between a history of sexual abuse experiences and perpetration of sexual abuse' (Proeve and Reilly 2007: 251).

Mendel (1995: 207) suggests that victimized males who later perpetrate abuse are distinguished from those who do not abuse, by their inability to recognize and 'integrate' their abusive experience, and 'access the feelings of pain, loss, and vulnerability' which ensue; and 'work through the trauma' (ibid.: 214). In contrast, sexual offenders experience anger following their sexual victimization in childhood; and this is communicated through sexual aggression (ibid.). In this study, 'feelings of pain, loss and vulnerability' were evident in some offenders' narratives; the opportunity to understand those was not. As in Garrett's (2010: 682) research, offenders in this study experienced childhoods characterized by 'fear, suspense, anticipation, uncertainty, anger and rejection'; and recalled feeling 'unwanted and unloved', and 'isolated'(ibid. 682). One of the key themes in Garrett's research was the men's emphasis on 'trying to survive' (ibid.: 683). This, too, was an experience shared by some of the 40 men in this study. The narratives of those who spent childhoods in residential care, in particular, were characterized by attempts to survive the abuse they were experiencing. Indeed, as with Garrett's participants, for some men in this study: 'Survival was a full-time occupation...with most of their time being consumed with avoiding harm and day-to-day survival'. The impact of being in a 'constant state of expectation and threat' (ibid. 683) cannot be under-estimated. Childhood experiences have the potential to profoundly impact on future behavior (ibid.). For the men who participated in this research, childhood did not offer a place of safety. Neither was it a time when they were given the 
opportunity to thrive; develop a sense of self-worth; or a positive view of themselves in relation to others.

Some evidence suggests that child sexual abusers learn to use sex as a coping mechanism to lessen the pain of the CSA they experienced (Cortoni and Marshall 2001). This seems to apply to some of the 40 men in this research. As Garrett (2010: 684) notes, however: 'Unfortunately, using sex to alleviate the pain of childhood sexual abuse frequently involves another child'. Reducing levels of future victimization will present considerable challenges and require much energy. Many abusers will never be detected, nor will their victims be accounted for. In working with offenders who report experiencing CSA, it appears there might be some merit: in enabling them to understand their own childhood sexual victimization, and the 'pain' associated with that, however it manifests itself; and how that 'pain' might be resolved. 
APPENDIX 


\section{NAME:}

WING:

I am a member of a research team from a University which is undertaking a study to further understanding of child sexual abuse. This will involve individual interviews with prisoners. Therefore, I am writing to you to ask whether you would be willing to be interviewed as part of the research.

The study is independent and is no way connected to the Psychology Department at HMP X, or the Programmes currently run by the prison. The interview should take no more than one and a half hours and everything you tell me will be treated in the strictest confidence. Your name will not be placed on the questionnaire and your identity will not be revealed to anyone. When we have finished the interview the questionnaire will be placed in my briefcase and taken back to the University, where it will be securely locked away. When the research is completed, the questionnaire will be destroyed.

I have scheduled your interview as follows:

INTERVIEW DATE:

INTERVIEW TIME:

I will collect prisoners being interviewed at $8.30 \mathrm{am}$ or $1.30 \mathrm{pm}$ from the Centre: I will collect prisoners being interviewed at $10 \mathrm{am}$ or $3 \mathrm{pm}$ from their workplace.

Susan Roberts 


\section{APPENDIX B: INTERVIEW QUESTIONS}

- To what extent do you see a link between your own family experiences as a child and your offences against children?

- To what extent do you see a link between your relationship with childhood friends, inside and outside school, and your offences against children?

- To what extent do you see a link between your relationship with any significant adult(s) outside your immediate childhood family and your offences against children?

- Can you tell me whether you were sexually abused as a child?

- Were you physically abused as a child?

- How old were you when you first became aware that you were sexually attracted to children?

- How do you think you came to be sexually attracted to children?

- In what ways did you find children sexually attractive? 
APPENDIX C: CONSENT FORM - TAPED INTERVIEW

UNIVERSITY OF WALES

RESEARCH PROJECT

I HAVE AGREED TO PARTICIPATE IN A TAPED INTERVIEW.

DATE:

SIGNATURE:

\section{UNIVERSITY OF WALES}

RESEARCH PROJECT

I HAVE AGREED TO PARTICIPATE IN A TAPED INTERVIEW.

DATE:

SIGNATURE:

\section{UNIVERSITY OF WALES}

RESEARCH PROJECT

I HAVE AGREED TO PARTICIPATE IN A TAPED INTERVIEW.

DATE:

SIGNATURE:

\section{UNIVERSITY OF WALES}

RESEARCH PROJECT

I HAVE AGREED TO PARTICIPATE IN A TAPED INTERVIEW.

DATE:

SIGNATURE: 


\section{Bibliography}

Abel, G.G., Gore, D.K., Holland, C.L. and Camp, N. (1989) The measurement of the cognitive distortions of child molesters, Annals of Sex Research, 2: 135-152.

Abel, G.G., Becker, J.V. and Cunningham-Rathner, J. (1984) Complications, consent, and cognitions in sex between children and adults, International Journal of Law and Psychiatry, 7: 89-103.

Abracen, J., and Looman, J. (2005) Developments in the assessment and treatment of sexual offenders: looking backward with a view to the future. Journal of Interpersonal Violence, 20: 12-19.

Afifi, T.O., MacMillan, H., Cox, B.J., Asmundson, G.J.G., Stein, M.B. and Sareen, J. (2009) Mental health correlates of intimate partner violence in marital relationships in a nationally representative sample of males and females, Journal of Interpersonal Violence, 24: 13981417.

Alaggia, R. (2004) Many ways of telling: expanding conceptualizations of child sexual abuse disclosure Child Abuse \& Neglect, 28: 1213-1227.

Alexander, P.C., Teti, L. and Anderson, C.L. (2000) Childhood sexual abuse history and role reversal in parenting, Child Abuse \& Neglect, 24: 829-838.

Almond, T. (2014) Working with children and young people with harmful sexual behaviours: exploring impact in practitioners and sources of support', Journal of Sexual Aggression, 20: 333-353.

Allnock, D. (2010) Children and young people disclosing sexual abuse: An introduction to the research, Available at: http://www.childmatters.org.nz/file/Diploma-Readings/Block2/Sexual-Abuse/3.4-children-and-young-people-disclosing-sexual-abuse-updated.pdf (Accessed 15.6.15). 
Allnock, D., Bunting, L., Price, A., Morgan-Klein, N., Ellis, J., Radford, L. and Stafford, A. (2009) Sexual abuse and therapeutic services for children and young people: the gap between provision and need, London: NSPCC.

Amen, T.M. (2002) An investigation of the psychological consequences to sex offender treatment providers, Doctoral dissertation, USA: Sam Houston State University.

American Psychiatric Association (2000) (4 ${ }^{\text {th }}$ Edn.) Diagnostic and statistical manual of mental disorders, Washington DC: American Psychiatric Association.

Aries, P. (1986) Centuries of Childhood: A Social History of Family Life, Harmondsworth: Penguin.

Armstrong, L. (2000) What happened when women said 'incest' in C. Itzin, (Ed.) (2000) Home Truths About Child Sexual Abuse, pp.27-48, New York: Routledge.

Babatsikos, G. and Miles, D. (2015) How Parents Manage the Risk of Child Sexual Abuse: A Grounded Theory, Journal of Child Sexual Abuse, 24: 55-76.

Back, S.E. (2003) Child sexual and physical abuse among college students in Singapore and the United States, Child Abuse \& Neglect, 27: 1259-1275.

Bacon, H. and Richardson, S. (2000) Child sexual abuse and the continuum of victim disclosure, in C. Itzin, (Ed.) (2000) Home Truths About Child Sexual Abuse, (pp.235-276), Abingdon: Routledge.

Bader, S.M., Scalora, M.J., Casady, T.K and Black, S. (2008) Female sexual abuse and criminal justice intervention: A comparison of child protective service and criminal justice samples, Child Abuse \& Neglect, 32: 111-119.

Bagley, C., Wood, M. and Young, L. (1994) Victim to abuser: mental health and behavioural sequels of child sexual abuse in a community survey of young adult males, Child Abuse \& Neglect, 18: 683-697. 
Baker, A.W. and Duncan, S.P. (1985) Child sexual abuse: A study of prevalence in Great Britain, Child Abuse \& Neglect, 9: 457-467.

Bandura, A. (1973) Aggression: A Social Learning Analysis, Englewood Cliffs, NJ: Prentic Hall.

Banning, A. (1989) Mother-son incest: Confronting a prejudice, Child Abuse \& Neglect, 13: 563-570.

Barnardo's (2012) Tackling child sexual exploitation: Helping local authorities to develop effective responses, Ilford: Barnardo’s.

Barnes, J.E., Noll, J.G., Putnam, F.W. and Trickett, P.K. (2009) Sexual and physical revictimisation among victims of severe childhood sexual abuse, Child Abuse \& Neglect, 33: 412-420.

Barter, C. (2003) Abuse of children in residential care, London: NSPCC.

Bascelli, E., Crisma, M., Paci, D. and Romito, P. (2004) Adolescents who experienced sexual abuse: fears, needs and impediments to disclosure, Child Abuse \& Neglect, 28: 1035-1048.

BBC News (2016) Kincora Boys' Home: Inquiry to examine abuse claims, Available at: http://www.bbc.co.uk/news/uk-northern-ireland-36413745 (Accessed 31.5.16).

BBC News (2015) Historical child abuse: Key investigations, Available at: http://www.bbc.co.uk/news/uk-28194271, (Accessed 28.4.16).

BBC News (2015) Reports of child abuse rising sharply, NSPCC says, Available at: http://www.bbc.co.uk/news/uk-33160361, (Accessed 17.6.15)

BBC News (2010) Vanessa George: 'Little Ted's was 'ideal' place for Vanessa George abuse', Available at: http://www.bbc.co.uk/news/uk-england-devon-11682161 (Accessed 29.2.16). 
BBC News (2001) Timeline: The Sarah Payne Tragedy, Available at: http://news.bbc.co.uk/1/hi/england/1703534.stm (Accessed 8.4.16).

Beech, A.R., Craig, L.A. and Browne, K.D. (2009) Assessment and Treatment of Sex Offenders: A Handbook, UK: Wiley-Blackwell.

Beech, A., Fisher, D., Beckett, R., and Fordham, A. (1998) An Evaluation of the Prison Sex Offender Treatment Programme, Research Findings, 79: 1-4, London: Home Office.

Beitchman, J.H., Zucker, K.J., Hood, J.E., daCosta, G.A., Akman, D., and Cassavia, E. (1992) A review of the long-term effects of child sexual abuse, Child Abuse \& Neglect, 16: 101-18.

Bennett, N. and O’Donohue, W. (2014) The Construct of Grooming in Child Sexual Abuse: Conceptual and Measurement Issues, Journal of Child Sexual Abuse, 23: 957-976.

Bentovim, A. (2002) Preventing sexually abused young people from becoming abusers, and treating the victimization experiences of young people who offend sexually, Child Abuse \& Neglect, 26: 661-678.

Bentovim, A. and Williams, B. (1998) Children and adolescents: victims who become perpetrators, Advances in Psychiatric Treatment, 4: 101-107.

Berliner, L. and Conte, J.R. (1995) The effects of disclosure and intervention on sexually abused children, Child Abuse \& Neglect, 19: 371-384.

Berliner, L. and Conte J. (1990) The process of victimization: the victims' perspective, Child Abuse \& Neglect, 14: 29-40.

Black, D.A., Heyman, R.E. and Smith Slep, A.M. (2001) Risk factors for child sexual abuse, Aggression and Violent Behavior, 6: 203-229. 
Blanchard, R., Lykins, A.D., Wherrett, D., Kuban, M.E., Cantor, J.M., Blak, T., Dickey, R. and Klassen, P.E. (2009) Pedophilia, Hebephilia, and the DSM-V, Archives of Sexual Behaviour, 38: 335-350.

Bogaerts, S., Declercq, F., Vanheule, S. and Palmans, V. (2005) Interpersonal Factors and Personality Disorders as Discriminators Between Intra-Familial and Extra-Familial Child Molesters, International Journal of Offender Therapy and Comparative Criminology, 49: 4862.

Boney-McCoy, S. and Finkelhor, D. (1995) Prior victimization: A risk factor for child sexual abuse and for PTSD-related symptomology among sexually abused youth, Child Abuse \& Neglect, 19: 1401-1421.

Boroughs, D.S. (2004) Female sexual abusers of children, Children and Youth Services Review, 26: 481-487.

Bourke, A., Doherty, S., McBride, O., Morgan, K. and McGee H. (2014) Female perpetrators of child sexual abuse: characteristics of the offender and victim, Psychology, Crime \& Law, 20: 769-780.

Boyd, C. (2006) Young people who sexually abuse: Key issues, Australian Centre for the Study of Sexual Assault (ACSSA) Wrap, No.3 December 2006, Available at: http://www.aifs.gov.au.acssa/pubs/wrap/w3.html (Accessed 13.6.16)

Brayford, J. and Roberts, S. (2012) 'Female sexual offending: exploring current knowledge' in Brayford, J., Cowe, F. and Deering, J. (Eds.) Sex Offenders: Punish, Help, Change or Control? Theory, Policy and Practice Explored, (pp. 90-108), Oxford: Routledge.

Briere, J. and Runtz, M. (1990) Differential adult symptomatology associated with three types of child abuse histories, Child Abuse \& Neglect, 14: 357-364.

Briggs F. (1995) From Victim to Offender: How Child Sexual Abuse Victims Become Offenders, NSW Australia: Allen \& Unwin. 
Briggs, F. and Hawkins, R.M.F. (1996) A comparison of the childhood experiences of convicted male child molesters and men who were sexually abused in childhood and claimed to be non-offenders, Child Abuse \& Neglect, 20: 221-233.

Briggs, F. and Hawkins, R.M.F. (1995) Protecting boys from the risk of sexual abuse, Early Childhood Development and Care, 110: 19-32.

British Society of Criminology (2015) Statement of Ethics, Available at: http://www.britsoccrim.org/documents/BSCEthics2015.pdf (Accessed 21.6.16)

Bromberg, D.S. and Johnson, B.T. (2001) Sexual Interest in Children. Child Sexual Abuse, and Psychological Sequelae for Children, Psychology in the Schools, 38: 343-355.

Brown, S., Brady, G., Franklin, A., Bradley, L., Kerrigan, N. and Sealey, C. (2016) Child Sexual Abuse ad Exploitation: Understanding Risk and Vulnerability, London: Early Intervention Foundation.

Brown, J., O’Donnell, T. and Erooga, M. (2011) Sexual Abuse: A public health challenge, London: NSPCC.

Browne, A. and Finkelhor, D. (1986) Impact of Child Sexual Abuse: A Review of the Research, Psychological Bulletin, 99: 66-77.

Brownmiller, S. (1993) (2 ${ }^{\text {nd }}$ edition) Against Our Will: Men, Women and Rape, US: Ballantine.

Bryman, A. (2008) (3 ${ }^{\text {rd }}$ ed.) Social Research Methods, Oxford: Oxford University Press.

Buchanan, A. (1996) Cycles of Child Maltreatment, Chichester: Wiley.

Buckley, K. (1995) 'Masculinity, the probation service and the causes of offending behaviour' in May, T. and Vass, A. (Eds.) Working with offenders. London: Sage.

Bunting, L. (2007) 'Dealing with a Problem that Doesn't Exist? Professional responses to 
Female Perpetrated Child Sexual Abuse’, Child Abuse Review, 16: 252-267.

Bunting, L. (2005) Females who sexually offend against children: Responses of the child protection and criminal justice systems, London: NSPCC.

Burkett, L.P. (1991) Parenting behaviours of women who were sexually abused as children in their family of origin, Family Process, 30: 421-434.

Burton, D.L. (2003) Male adolescents: Sexual victimisation and subsequent sexual abuse, Child and Adolescent Social Work Journal, 20: 277-296.

Burton, D.L., Duty, K.J. and Leibowitz, G.S. (2011) Differences between Sexually Victimised and Nonsexually Victimised Male Adolescent Sexual Abusers: Developmental Antecedents and Behavioral Comparisons, Journal of Child Sexual Abuse, 20: 77-93.

Burton, D.L., Miller, D.L. and Shill, C.T. (2002) A social learning theory comparison of the sexual victimization of adolescent sexual offenders and nonsexual offending male delinquents, Child Abuse \& Neglect, 26: 893-907.

Caffaro, J.V. and Conn-Caffaro, A. (2005) Treating sibling abuse families, Aggression \& Violence, 10: 604-623.

Calder, M.C. (2004) The Internet: Potential problems and pathways to hands-on sexual offending, in M.C. Calder (Ed.) Child sexual abuse and the Internet: tackling the new frontier (pp.1-24) Lyme Regis, UK: Russell House.

Calder, J., McVean, A. and Yang, W. (2010) History of abuse and current suicidal ideation: results from a population based survey, Journal of Family Violence, 25: 205-214.

Caldwell, M.F. (2007) Sexual Offense Adjudication and Sexual Recidivism among Juvenile Offenders, Sex Abuse, 19: 107-113.

Carlson, B.E., Maciol, K. and Scheider, J. (2006) Sibling incest: Reports from forty-one survivors, Journal of Child Sexual Abuse, 15: 4. 
Cawson, P., Wattam, C., Brooker, S. and Kelly, G. (2000) Child maltreatment in the United Kingdom: A study of the prevalence of abuse and neglect, London: NSPCC.

CEOP (2012) A Picture of Abuse: A thematic assessment of the risk of contact child sexual abuse posed by those who possess indecent images of children, Available at: https://www.ceop.police.uk/Documents/ceopdocs/CEOP\%20IIOCTA\%20Executive\%20Summ ary.pdf London: CEOP (Accessed 21.6.16).

Chandy, J.M., Blum, R.W. and Resnick, M.D. (1996) Gender-Specific Outcomes for Sexually Abused Adolescents, Child Abuse \& Neglect, 20: 1219-1231.

Children's Commissioner for England (2015) Protecting Children from Harm: A critical assessment of child sexual abuse in the family network for England and priorities for action, Available at:

http://www.childrenscommissioner.gov.uk/sites/default/files/publications/Protecting\%20child ren\%20from\%20harm\%20-\%20executive\%20summary_0.pdf (Accessed 23.8.16).

Christopher, K., Lutz-Zois, C.J., and Reinhardt, A.R. (2007) Female Sexual Offenders: Personality Pathology as a Mediator of the Relationship between Childhood Sexual Abuse History and Sexual Abuse Perpetration against Others, Child Abuse \& Neglect, 31: 871-883.

Colton, M. (2002) Factors Associated with Abuse in Residential Child Care Institutions, Children \& Society, 16: 33-44.

Colton, M. and Vanstone, M. (1998) Sexual Abuse by Men who Work with Children: An Exploratory Study, British Journal of Social Work, 28: 511-523.

Colton, M. and Vanstone, M. (1996) Betrayal of Trust: Sexual Abuse by Men who Work with Children, London: Free Association Books.

Colton, M., Vanstone, M. and Walby, C. (2002) Victimization, Care and Justice: Reflections on the Experiences of Victims/Survivors Involved in Large-scale Historical Investigations of Child Sexual Abuse in Residential Institutions, British Journal of Social Work, 32: 541-551. 
Colton, M., Roberts, S. and Vanstone, M. (2012) Learning lessons from men who have sexually abused children, The Howard Journal of Criminal Justice, 51: 79-93.

Colton, M., Roberts, S. and Vanstone, M. (2010) Sexual Abuse by Men who Work with Children, Journal of Child Sexual Abuse, 19: 345-364.

Colton, M., Roberts, S. and Vanstone, M. (2009) Child Sexual Abusers' Views on Treatment: A Study of Convicted and Imprisoned Adult Male Offenders, Journal of Child Sexual Abuse, 18: 320-338.

Connell, R.W. and Messerschmidt, J.W. (2005). Hegemonic Masculinity: Rethinking the Concept, Gender and Society, 19: 829-59.

Connolly, M. and Woollons, R. (2008) Childhood Sexual Experience and Adult Offending: An Exploratory Comparison of Three Criminal Groups, Child Abuse Review, 17: 119-132.

Conte, J.R., Wolf, S. and Smith, T. (1989) What Sexual Offenders Tell Us About Prevention Strategies, Child Abuse \& Neglect, 13: 293-301.

Conte, J.R. and Schuerman, J.R. (1987a) Factors Associated With An Increased Impact of Child Sexual Abuse, Child Abuse \& Neglect, 11: 201-211.

Conte, J.R and Schuerman, J.R. (1987b) The effects of sexual abuse on children: A multidimensional view, Journal of Interpersonal Violence, 2: 380-390.

Cortoni, F. and Marshall, W. (2001) Sex as a coping strategy and its relationship to juvenile sexual history and intimacy in sexual offenders, Sexual Abuse: A Journal of Research and Treatment, 12: 39-59.

Cossar, J., Brandon, M., Bailey, S., Beldersen, P., Biggart, L. and Sharpe, D. (2013) 'It takes a lot to build trust', Recognition and Telling: Developing Earlier Routes to Help for Children and Young People, Available at:

https://www.uea.ac.uk/documents/3437903/0/OCC+Recognition+and+Telling+report.pdf/92 3aec11-0221-4d54-8c77-f83189e9e02d (Accessed 24.8.2016). 
Cossins, A. (2008) 'Restorative Justice and Child Sex Offences: The Theory and the Practice', British Journal of Criminology, 48: 359-378.

Cossins, A. (2000) Masculinities, sexualities and child sexual abuse, The Hague: Kluwer Law International.

Cossins, A. and Plummer, M. (2016) Masculinity and Sexual Abuse: Explaining the Transition from Victim to Offender, Men and Masculinities, 1-26, doi: 10.1177/1097184X16652655.

Cowburn, M. (2005) Confidentiality and public protection: Ethical dilemmas in qualitative research with adult male sex offenders, Journal of Sexual Aggression, 11: 49-63.

Cowburn, M. (1998) A Man’s World: Gender Issues in Working with Male Sex Offenders in Prison, Howard Journal of Criminal Justice, 37: 234-251.

Coy, M. (2008) 'Young Women, Local Authority Care and Selling Sex: Findings from Research’, British Journal of Social Work, 38: 1408-1424.

Coyle, A. and Wright, C. (1996) Using the counselling interview to collect research data on sensitive topics, Journal of Health Psychology, 1: 431-440.

Crabtree, D.A. (2002) Vicarious traumatisation in therapists who work with juvenile sex offenders, Doctoral dissertation, USA: Pace University.

Craissati, J. and Beech, A. (2004) The Characteristics of a Geographical Sample of Convicted Rapists: Sexual Victimization and Compliance in Comparison to Child Molesters, Journal of Interpersonal Violence, 19: 371-388.

Craissati, J., McClurg, G. and Browne, K. (2002) Characteristics of Perpetrators of Child Sexual Abuse Who Have Been Sexually Victimized as Children, Sexual Abuse: A Journal of Research and Treatment, 14: 225-239. 
Craissati, J., McClurg, G. and Falla, S. (1999) The Challenge Project. Child Sexual Abusers in S.E. London: profile, treatment and outcome, Internal document, Oxleas NHS Trust.

Craissati, J. and McClurg, G. (1996) 'The Challenge Project: Perpetrators of child sexual abuse in S.E. London', Child Abuse \& Neglect, 21: 637-648.

Criminal Justice Act ( 2003) London: Stationery Office.

Crisma, M., Bascelli, E., Paci, D. and Romito, P. (2004) Adolescents who experienced sexual abuse: Fears, needs and impediments to disclosure, Child Abuse \& Neglect, 28: 1035-1048.

CPS (2015) Violence against Women and Girls Crime Report 2014-2015, Available at: https://www.cps.gov.uk/publications/docs/cps_vawg_report_2014.pdf (Accessed 20.7.16)

Cupoli, J.M. and Sewell, P. (1988) One thousand fifty-nine children with a chief complaint of sexual abuse, Child Abuse \& Neglect, 12: 151-162.

Currie, J. and Widom, C.S. (2010) Long-Term Consequences of Child Abuse and Neglect on Adult Economic Well-Being, Child Maltreatment, 15: 111-120.

Cyr, M., Wright, J., McDuff, P. and Perron, A. (2002) Intrafamilial sexual abuse: brothersister incest does not differ from father-daughter and stepfather-stepdaughter incest, Child Abuse \& Neglect, 26: 957-973.

Daily Mail (2006) Female teacher jailed for sex with pupil, Available at: http://www.dailymail.co.uk (Accessed 12.11.10).

Dale, K. and Alpert, J. (2007) Hiding behind the cloth: Child sexual abuse and the Catholic Church, Child Abuse \& Neglect, 16: 59-74.

Day, A. (1999). Sexual offender views about treatment: a client survey. Journal of Child Sexual Abuse, 8: 93-103. 
Deering, R. and Mellor, D. (2011) An Exploratory Study of the Self-Reported Impact of Female-Perpetrated Childhood Sexual Abuse, Journal of Child Sexual Abuse, 20: 58-76.

de Jong, R., Alink, L., Bijleveld, C., Finkenauer, C. and Hendriks, J. (2015) Transition to adulthood of child sexual abuse victims, Aggression and Violent Behavior, 24: 175-187.

Denney, A.S. and Tewksbury, R. (2013) How to Write a Literature Review, Journal of Criminal Justice Education, 24: 218-234.

Denov, M. (2003) 'To a safer place? Victims of sexual abuse by females and their disclosures to professionals', Child Abuse \& Neglect, 27: 47-61.

Denov, M. (2004a) 'The long-term effects of child sexual abuse by female perpetrators: a qualitative study of male and female victims', Journal of Interpersonal Violence, 19: 11371156.

Denov, M.S. (2004b) Perspectives on Female Sex Offending: A Culture of Denial, Aldershot: Ashgate.

Denscombe, M. (2003) The Good Research Guide, Maidenhead: Open University Press.

Department of Health and Home Office (2006) The needs and effective treatment of young people who sexually abuse: current evidence, Available at:

http://www.rcpsych.ac.uk/pdf/Needs\%20and\%20treatment\%20of\%20YP\%20who\%20sexuall y\%20abuse.pdf (Accessed 31.7.14).

Dhawan, S. and Marshall, W. (1996) Sexual abuse histories of sexual offenders Sexual Abuse: A Journal of Research and Treatment, 8: 7-15.

DiLillo D., Tremblay, G.C. and Peterson L. (2000) Linking childhood sexual abuse and abusive parenting: the mediating role of maternal anger, Child Abuse \& Neglect, 24: 767-779. 
Dobash, R. P. Carnie, J. and Waterhouse L. (1993) Child sexual abusers: Recognition and response, in L.Waterhouse (Ed.), Child abuse and child abusers (pp.113-135), London: Jessica Kingsley.

Dobson, A. (2003) Caught in the net, Care and Health, 11: 6-9.

Dodsworth, J. (2014) Sexual Exploitation, Selling and Swapping Sex: Victimhood and Agency, Child Abuse Review, 23: 185-199.

Doel, M. and Sawdon, S. (1999) The essential groupworker: Teaching and learning creative groupwork, London: Jessica Kingsley.

Durham, A. (2003) Young Men Living Through and with Child Sexual Abuse: A Practitioner Research Study, British Journal of Social Work, 33: 309-323.

Easton, S.D. (2013) Disclosure of Child Sexual Abuse Among Adult Male Survivors, Clinical Social Work Journal, 41: 344-355.

Edwards, R., Whittaker, M.K., Beckett, R., Bishopp, D. and Bates, A. (2012) Adolescents who have sexually harmed: An evaluation of a specialist treatment programme, Journal of Sexual Aggression, 18: 91-111.

Elliott, M. (1993) (Ed.) Female Sexual Abuse of Children: The Ultimate Taboo, UK: Longman.

Elliott, I.A. and Ashfield, S. (2011) The use of on-line technology in the modus operandi of female sex offenders, Journal of Sexual Aggression, 17: 92-104.

Elliott, I.A. and Beech, A.R. (2009) Understanding online child pornography use: Applying sexual offense theory to internet offenders, Aggression and Violent Behavior, 14: 180-193.

Elliot, M., Browne, K. and Kilcoyne, J. (1995) Child Sexual Abuse Prevention: What Offenders Tell Us, Child Abuse \& Neglect, 19: 579-594. 
Ennew, J. (1986) The Sexual Exploitation of Children, Cambridge: Polity Press.

Epps, K. and Fisher, D. (2004) A review of the research literature on young people who sexually abuse, in G. O’Reilly, W. Marshall, A. Carr and R.C. Beckett (Eds.) The Handbook of Clinical Intervention with Young People who Sexually Abuse, (pp.62-102), New York: Psychology Press, Available at:

https://books.google.co.uk/books?hl=en\&lr=\&id=xMV4AgAAQBAJ\&oi=fnd\&pg=PA62\&d $\underline{q}=$ epps+and+fisher+2004\&ots=dViEwT9eQk\&sig=20VkjsOygsRhdcKJEfNxLgApiAM\#v= onepage\&q\&f=false (Accessed 5.9.16)

Erooga, M., Allnock, D. and Telford, P. (2012) Towards Safer Organisations II: Using the perspectives of convicted sex offenders to inform organisational safeguarding of children, Available at:

https://www.nspcc.org.uk/globalassets/documents/research-reports/towards-saferorganisations-2012-report.pdf (Accessed 5.9.16).

Erooga, M. and Masson, H. (2006) (Eds.) Children and Young People who Sexually Abuse Others: Current developments and practice responses, $2^{\text {nd }}$ Edition, Abingdon: Routledge.

Etherington, K. (1997) Maternal Sexual Abuse of Males, Child Abuse Review, 6: 107-117. Faller, K. (1995) A Clinical Sample of Women who have Sexually Abused Children, Journal of Child Sexual Abuse, 4: 13-30.

Faller, K. C. (1989a) Why Sexual Abuse? An Exploration of the Intergenerational Hypothesis, Child Abuse \& Neglect, 13: 543-548.

Faller, K. C. (1989b) Characteristics of a clinical sample of sexually abused children: How boy and girl victims differ, Child Abuse \& Neglect, 13: 281-291.

Faller, K.C. (1988) Criteria for judging the credibility of children's statements about their sexual abuse, Child Welfare, 68: 389-401. 
Fallon, B., Trocme, N., Fluke, J., Maclaurin, B., Tonmyr, L. and Yuan, Y.Y. (2010) Methodological challenges in measuring child maltreatment, Child Abuse \& Neglect, 34: 7079.

Falshaw, L., Browne, K.D. and Hollin, C.R. (1996) Victim to offender: A review, Aggression and Violent Behaviour, 1: 389-404.

Fehrenbach, P.A. and Monastersky, C. (1988) Characteristics of female adolescent sexual offenders, American Journal of Orthopsychiatry, 48: 148-178.

Feiring, C., Taska, L. and Lewis, M. (2002) Adjustment following sexual abuse discovery: the role of shame and attributional style, Developmental Psychology, 38: 79-92.

Fergusson, D.M., McLeod, G.F.H. and Horwood, L.J. (2013) Childhood sexual abuse and adult developmental outcomes: Findings from a 30-year longitudinal study in New Zealand, Child Abuse \& Neglect, 37: 664-674.

Finkelhor, D. (1995) The Victimization of Children: A Developmental Perspective, American Journal of Orthopsychiatry, 65: 177-193.

Finkelhor, D. (1994) The International Epidemiology of Child Sexual Abuse, Child Abuse \& Neglect, 18: 409-417.

Finkelhor, D. (1987) The Trauma of Child Sexual Abuse: Two Models, Journal of Interpersonal Violence, 2: 348-366.

Finkelhor, D. (1984) Child Sexual Abuse: New Theory \& Research, New York, The Free Press.

Finkelhor, D. (1982) 'Sexual abuse: a sociological perspective', Child Abuse and Neglect, 6: 95-102.

Finkelhor, D. (1980) Risk factors in the sexual victimisation of children', Child Abuse and Neglect, 4: 265-273. 
Finkelhor, D. (1979) Sexually Victimised Children, New York: The Free Press.

Finkelhor, D., Ormrod, R. and Chaffin, M. (2009) Juveniles who commit sex offenses against minors, Office of Justice Programs, Washingon, DC: U.S. Department of Justice.

Finkelhor, D., Moore, D., Hamby, S.L. and Straus, M.A. (1997) Sexually Abused Children in a National Survey of Parents: Methodological Issues, Child Abuse \& Neglect, 21: 1-9.

Finkelhor, D., Hotaling, G., Lewis, I.A. and Smith, C. (1990) Sexual abuse in a national survey of adult men and women: prevalence, characteristics, and risk factors, Child Abuse \& Neglect, 14: 19-28.

Finkelhor, D., Williams, L.M., Burns, N. and Kalinowski, M. (1988a) Sexual abuse in day care: A national study, Family Research Laboratory: University of New Hampshire.

Finkelhor, D., Meyer Williams, L. and Burns, N. (1988b) Nursery Crimes: Sexual Abuse in Day Care, London: Sage.

Finkelhor, D., and Korbin, J. (1988c) Child abuse as an international issue, Child Abuse \& Neglect, 12: 3-23.

Finkelhor, D. with Araji, S. (1986) A sourcebook on child sexual abuse, London: Sage.

Finkelhor, D. and Browne, A. (1985) The Traumatic Impact of Child Sexual Abuse: A Conceptualization, American Journal of Orthopsychiatry, 55: 530-541.

Finkelhor, D. and Russell, D. (1984) Women as perpetrators, in D. Finkelhor (Ed.) Child Sexual Abuse, New Theory and Research, (pp. 171-185), New York: The Free Press.

Fischer, D.G. and McDonald, W.L. (1998) Characteristics of intrafamilial and extrafamilial child sexual abuse, Child Abuse \& Neglect, 22: 915-929. 
Fitzpatrick, M., Carr, A., Dooley, B., Flanagan-Howard, R., Flanagan, E., Tierney, K., White, M., Daly, M., Shevlin, M. and Egan, J. (2010) Profiles of adult survivors of severe sexual, physical and emotional institutional abuse in Ireland, Child Abuse Review, 19: 387-404.

Fleming, J., Mullen, P.E., Sibthorpe, B. and Bammer, G. (1999) The Long-Term Impact of Childhood Sexual Abuse in Australian Women, Child Abuse \& Neglect, 23: 145-159.

Fleming, J., Mullen, P.E., and Bammer, G. (1997) A study of potential risk factors for sexual abuse in childhood, Child Abuse \& Neglect, 21: 49-58.

Fondacaro, K.M., Holt, J.C. and Powell, T.A. (1999) Psychological Impact of Childhood Sexual Abuse on Male Inmates: The Importance of Perception, Child Abuse \& Neglect, 23: 361-369.

Fontes, L.A. and Plummer, C. (2010) Cultural Issues in Disclosures of Child Sexual Abuse, Journal of Child Sexual Abuse, 19: 491-518.

Freeman, N.J. and Sandler, J.C. (2008) Female and Male Sex Offenders: A Comparison of Recidivism Patterns and Risk Factors, Journal of Interpersonal Violence, 23: 1394-1413.

Freel, M. (2003) Child Sexual Abuse and the Male Monopoly: An Empirical Exploration of Gender and a Sexual Interest in Children, British Journal of Social Work, 33: 481-498.

Frei, A. (2008) Editorial: Media Consideration of Sex Offenders: How Community Response Shapes a Gendered Perspective, International Journal of Offender Therapy and Comparative Criminology, 52: 495-498.

Freidrich, A., Urquiza, A., and Beilke, R. (1986) Behavior problems in sexually abused young children, Journal of Pediatric Psychology, 11, 47-57.

Friendship, C., Mann, R. and Beech, A. (2003) The Prison-based Sex Offender Treatment Programme - an Evaluation, Home Office Research Findings 205, London: Home Office. 
Fromuth, M.E. and Burkhart B.R. (1987) Childhood Sexual Victimisation among College Men: Definitional and Methodological Issues, Violence and Victims, 2: 241-253.

Frost, A. (2004) Therapeutic Engagement Styles of Child Sexual Offenders in a Group Treatment Program: A Grounded Theory Study. Sexual Abuse: A Journal of Research and Treatment, 16: 191-208.

Gallagher, B. (1999) The Abuse of Children in Public Care, Child Abuse Review, 8: 357-365.

Gannon, T.A. and Cortoni, F. (eds.) (2010) Female Sexual Offenders: Theory, Assessment and Treatment, UK: Wiley-Blackwell.

Gannon, T.A., Rose, M.R., and Ward, T. (2008) A Descriptive Model of the Offense Process for Female Sexual Offenders. Sexual Abuse: A Journal of Research and Treatment, 20: 352374.

Garrett, L.H. (2010) Childhood Experiences of Incarcerated Male Child Sexual Abusers, Issues in Mental Health Nursing, 31: 679-685.

Genders, E. and Player, E. (1995) Grendon: A Study of a Therapeutic Prison, Oxford: Oxford University Press.

Gilbert, N. (Ed.) (1993), Researching Social Life, London: Sage Publications.

Gill, M. and Tutty, L.M. (1999) Male survivors of childhood sexual abuse: A qualitative study, Journal of Child Sexual Abuse, 6: 31-47.

Gilligan, P. and Akhtar, S. (2006) Cultural Barriers to the Disclosure of Child Sexual Abuse in Asian Communities: Listening to What Women Say, British Journal of Social Work, 36: 1361-1377.

Gittens, D. (2004) The historical construction of childhood, in Kehily, M.J. (Ed.) (2004) An Introduction to Childhood Studies, Maidenhead, Open University Press. 
Glaser, B.G. and Strauss, A.L. (1967) The Discovery of Grounded Theory: Strategies for Qualitative Research, Chicago, Aldine.

Glasser, M., Kolvin, I., Campbell, D., Glasser, A., Leitch, I., and Farrelly, S. (2001) Cycle of child sexual abuse: Links between being a victim and becoming a perpetrator, British Journal of Psychiatry, 179: 482-494.

Gomes-Schwartz, B., Horowitz, J.M. and Cardarelli, A.P. (1990) Child sexual abuse: the initial effects, Newbury Park, CA: Sage.

Goodman-Brown, T.B., Edelstein, R.S., Goodman, G.S., Jones, D.P.H. and Gordon, D.S. (2003) 'Why children tell: a model of children's disclosure of sexual abuse', Child Abuse \& Neglect, 27: 525-540.

Goodwin, J., Cormier, L. and Owen, J. (1983) Grandfather-Granddaughter Incest: A Trigenerational View, Child Abuse \& Neglect, 7: 163-170.

Goodyear-Brown, P. (Ed.) (2012) Handbook of Child Sexual Abuse: Identification, Assessment and Treatment, New Jersey: Wiley.

GOV. UK (2014) The Data Protection Act, Available at: https://www.gov.uk/dataprotection/the-data-protection-act (Accessed 16.10.14)

Grattagliano, I., Owens, J.N., Morton, R.J., Campobasso, C.P., Carabellese, F. and Catanesi, R. (2012) Female sexual offenders: Five Italian case studies, Aggression and Violent Behavior, 17: 180-187.

Grayston, A., and DeLuca, R. (1999) Female Perpetrators of Child Sexual Abuse: A review of the clinical and empirical literature, Aggression and Violent Behavior, 4: 93-106.

Groth, A.N. (1979) Sexual Trauma in the Life Histories of Rapists and Child Molesters, Victimology: An International Journal, 4: 10-16.

Grubin, D (1998) Sex Offending Against Children: understanding the risk, Police Research 
Series Paper 99, London: Home Office Research, Development and Statistics Directorate.

Hackett, S. (2014) Children and young people with harmful sexual behaviours, Research Review, Research in Practice, Available at:

http://bedfordscb.proceduresonline.com/pdfs/ch_yp_harmful_sexual_behav.PDF (Accessed 5.9.16).

Hackett, S. (2004) What works for children and young people with harmful sexual behaviours? Barnardo’s Policy and Research Unit, Available at:

http://www.barnardos.org.uk/what_works_for_children_and_young_people_with_harmful_se xual_behaviours___summary_1_.pdf (Accessed 6.7.15).

Hackett, S. (2001) Facing the Future: A Guide for Parents of Young People Who Have Sexually Abused, UK: Russell House Publishing Ltd.

Hackett, S., Carpenter, J., Patsios, D. and Szilassy, E. (2013) Interprofessional and interagency training for working with young people with harmful sexual behaviours: An evaluation of outcomes, Journal of Sexual Aggression, 19,3: 329-344.

Hackett, S., Masson, H., Balfe, M. and Phillips, J. (2013) Individual, Family and Abuse Characteristics of 700 British Child and Adolescent Sexual Abusers, Child Abuse Review, 22: 232-245.

Hackett, S. and Masson, H. (2006) Young People who have Sexually Abused: What do they (and their parents) want from Professionals? Children \& Society, 20: 183-195.

Hall, D.K., Mathews, F. and Pearce, J. (2002) Sexual behaviour problems in sexually abused children: a preliminary typology, Child Abuse \& Neglect, 26: 89-312.

Hanson, R.K. (2014) Treating sexual offenders: how did we get here and where are we headed? Journal of Sexual Aggression: An international, interdisciplinary forum for research, theory and practice, 20: 3-8. 
Hanson, R.K. and Slater, S. (1988) Sexual victimisation in the history of child sexual abusers: a review, Annals of Sex Research, 1: 485-499.

Harris, D.A., Mazerolle, P. and Knight, R.A. (2009) Understanding Male Sexual Offending: A Comparison of General and Specialist Theories, Criminal Justice and Behavior, 36: 10511069.

Harrison, C. (2006) Cyberspace and Child Abuse Images: A Feminist Perspective, Affilia: Journal of Women and Social Work, 21: 365-379.

Harrison, P.A., Fulkerson, J.A. and Beebe, T.J. (1997) Multiple substance abuse among adolescent physical and sexual abuse victims, Child Abuse \& Neglect, 21: 529-539.

Hatch, J. (2005) Adolescents who sexually abuse their siblings; A study of family factors and victim selection, University of Melbourne: Melbourne.

Haugaard, J.J. and Emery, R.E. (1989) Methodological issues in child sexual abuse research, Child Abuse \& Neglect, 13: 89-100.

Hayman, S. (1997) (Ed.) Child Sexual Abuse: Myth and Reality, Institute for the Study and Treatment of Delinquency: King’s College, London

Hershkowitz, I., Lamb, M.E. and Horowitz, D. (2007) Victimization of children with disabilities. American Journal of Orthopsychiatry 77: 629-635.

Hetherton, J. (1999) 'The Idealization of Women: Its role in the minimization of child sexual abuse by females, Child Abuse and Neglect 23: 161-174.

Hetherton, J. and Beardsall, L. (1998) Decisions and attitudes concerning child sexual abuse: Does the gender of the perpetrator make a difference to child protection professionals? Child Abuse and Neglect, 22: 1265-1253. 
Hickey, N., McCrory, E., Farmer, E. and Vizard, E. (2008) Comparing the developmental and behavioural characteristics of female and male juveniles who present with sexually abusive behaviour, Journal of Sexual Aggression, 14: 241-252.

Hilton, M.R., and Mezey, G.C. (1996) Victims and perpetrators of child sexual abuse, The British Journal of Psychiatry, 169: 408-415.

HM Government (2015) Working together to safeguard children: a guide to inter-agency working to safeguard and promote the welfare of children, London: Department for Education.

HM Government (2007) Action Plan on Sexual Violence \& Abuse, Available at:

http://webarchive.nationalarchives.gov.uk/+/http:/www.homeoffice.gov.uk/documents/Sexua l-violence-action-plan2835.pdf?view=Binary

(Accessed 6.7.15)

HMIC (2014) Crime recording: making the victim count, London: HMIC.

Home Office (2004) Children and Families: Safer from Sexual Crime, The Sexual Offences Act 2003, London: Home Office.

Houtepen, J.A.B.M., Sijtsema, J.J. and Bogaerts, S. (2014) From child pornography offending to child sexual abuse: A review of child pornography offender characteristics and risks for cross-over, Aggression and Violent Behavior, 19: 466-473.

Howe, D. (2008) The Emotionally Intelligent Social Worker, Basingstoke: Palgrave MacMillan.

Howitt, D. (1995) Paedophiles and Sexual Offences Against Children, Chichester: Wiley.

Hudson, K. (2005) Offending Identities: Sex offenders' perspectives on their treatment and management, Cullompton, Devon: Willan. 
Hudson, K. (2003) Offending Identities: Sex offenders' perspectives on their treatment and management, Unpublished PhD thesis, University of Wales, Cardiff.

Hughes-Jones, L. and Roberts, S. (2015) Closing the Gap: Confronting the Problem of Hotels as Venues for Child Sexual Exploitation, The Howard Journal of Criminal Justice, 54: 336351.

Hunter, J.A., Lexier, L.J, Goodwin, D.W., Browne, P.A. and Dennis, C. (1993) Psychosexual, attitudinal, and developmental characteristics of juvenile female sexual perpetrators in a residential treatment setting, Journal of Child and Family Studies, 2: 317326.

Itzin, C. (2000) (Ed.) Home Truths About Child Sexual Abuse, Abingdon: Routledge.

Jankowski, M.K., Leitenberg, H., Henning, K. and Coffey, P. (2005) Parental caring as a possible buffer against sexual revictimisation in young adult survivors of child sexual abuse, Journal of Traumatic Stress, 15: 235-244.

Jensen, T.K., Gulbrandsen, W., Mossige, S., Reichelt, S. and Tjersland, O.A. (2005) Reporting possible sexual abuse: A qualitative study on children's perspectives and the context for disclosure, Child Abuse \& Neglect, 29: 1395-1413.

Jespersen, A.F., Lalumiere, M.L. and Seto, M.C. (2009) Sexual abuse history among adult sex offenders and non-sex-offenders: A meta-analysis, Child Abuse \& Neglect, 33: 179-192.

Johnson, T.C. (1989) Female child perpetrators: Children who molest other children, Child Abuse \& Neglect, 13: 571-585.

Johnson, T.C. (1988) Child perpetrators - Children who molest other children: Preliminary Findings, Child Abuse \& Neglect, 12: 219-229.

Johnson, R. J., Ross, M. W., Taylor, W. C., Williams, M. L., Carvajal, R.I. and Peters, R. J. (2006) Prevalence of childhood sexual abuse among incarcerated males in county jail, Child Abuse \& Neglect, 30: 75-86. 
Jones, L., Bellis, M.A., Wood, S., and Hughes, K. (2012) Prevalence and risk of violence against children with disabilities: a systematic review and meta-analysis of observational studies. The Lancet July 2012, Available at:

http://press.thelancet.com/childrendisabilities.pdf (Accessed 25.6.15)

Joyce, P.A. (1997) Mothers of Sexually Abused Children and the Concept of Collusion: A Literature Review, Journal of Child Sexual Abuse, 6: 75-92.

Jutte, S.J., Bentley, H., Tallis,, D., Mayes, J., Jetha, N., O’Hagan, O., Brookes, H and McConnell, N. (2015) How safe are our children? The most comprehensive overview of child protection in the $U K$, Available at:

http://www.nspcc.org.uk/globalassets/documents/research-reports/how-safe-children-2015report.pdf (Accessed 25.6.15)

Kahan, B. (1997) The victim's perspective before, during and after the abuse, in S. Hayman (1997) (Ed.) Child Sexual Abuse: Myth and Reality (pp.6-12), Institute for the Study and Treatment of Delinquency: King’s College, London.

Kamphuis, J. H., De Ruiter, C., Janssen, B., and Spiering, M. (2005) Preliminary Evidence for an Automatic Link Between Sex and Power Among Men Who Molest Children, Journal of Interpersonal Violence, 20: 1351-1365.

Kaplan, M.S. and Green, A. (1995) Incarcerated female sexual offenders: A comparison of sexual histories with eleven female nonsexual offenders, Sexual Abuse: A Journal of Research and Treatment, 7: 287-300.

Kaufman, J and Zigler, E. (1987) Do Abused Children Become Abusive Parents? American Journal of Orthopsychiatry, 57: 186-192.

Kear-Colwell, J., and Boer, D.P. (2000). The Treatment of Pedophiles: Clinical Experience and the Implications of Recent Research. International Journal of Offender Therapy and Comparative Criminology, 44: 593-605. 
Kehily, M.J. (ed.) (2004) An Introduction to Childhood Studies, Maidenhead: Open University Press.

Kehily, M.J. and Montgomery, H. (2003) Innocence and Experience in M. Woodhead and H. Montgomery (Eds.) Understanding Childhood: an interdisciplinary approach, UK: Wiley/Open University.

Kelly, R.J., Wood, J.J., Gonzalez, L.S., MacDonald, V. and Waterman, J. (2002) Effects of mother-son incest and positive perceptions of sexual abuse experiences on the psychosocial adjustment of clinic-referred men, Child Abuse \& Neglect, 26: 425-441.

Kendall-Tackett, K., Williams, L.M. and Finkelhor, D. (1993) Impact of Sexual Abuse on Children: A Review and Synthesis of Recent Empirical Studies, Psychological Bulletin, 113: 164-180.

Kendall-Tackett, K.A. and Simon, A.F. (1987) Perpetrators and their Acts: Data from 365 Adults Molested as Children, Child Abuse \& Neglect, 11: 237-245.

Kim, K., Trickett, P.K. and Puttnam, F.W. (2010) Childhood experiences of sexual abuse and later parenting practices among non-offending mothers of sexually abused and comparison girls, Child Abuse \& Neglect, 34: 610-622.

King, R. D. (2000) Doing Research in Prisons, in R.D. King and E. Wincup (Eds.) Doing Research on Crime and Justice, (pp.285-312), Oxford: Oxford University Press.

Kirkwood, A. (1993) The Leicestershire Inquiry 1992, Leicestershire County Council: Leicester.

Kitzinger, J. (2004) Framing Abuse: Media Influence and Public Understanding of Sexual Violence Against Children, London: Pluto Press.

Kitzinger, J. (1988) Defending innocence: Ideologies of childhood, Feminist Review, 28: 7787. 
Knight, C. and Modi, P. (2014) The use of emotional literacy with sexual offenders, Probation Journal, 61: 132-147.

Krug, R.S. (1989) Adult Male Report of Childhood Sexual Abuse by Mothers: Case Descriptions, Motivations and Long-Term Consequences, Child Abuse \& Neglect, 13: 111119.

Kubik, E.K., Hecker, J.E. and Righthand, S. (2003) Adolescent females who have sexually offended: Comparisons with delinquent adolescent female offenders and adolescent males who sexually offend, Journal of Child Sexual Abuse, 11: 63-83.

Kvale, S. (1996) Interviews: An Introduction to Qualitative Research Interviewing, Thousand Oaks, California: Sage.

La Fontaine, J. (1990) Child Sexual Abuse, Cambridge: Polity Press.

Lamb, S. (1999) (Ed.) New versions of victims: Feminists struggle with the concept, New York: New York University Press.

Lambie, I. and Johnson, E. (2016) "I Couldn't Do It to a Kid Knowing What it Did to Me”: The Narratives of Male Sexual Abuse Victims' Resiliency to Sexually Offending, International Journal of Offender Therapy and Comparative Criminology, 60: 897-918.

Lambie, I. and Price, M. (2015) Transitioning youth with sexually harmful behaviour back into the community, Journal of Sexual Aggression, 21: 244-265.

Lambie, I., Seymour, F., Lee, A. and Adams, P. (2002) Resiliency in the victim-offender cycle in in male sexual abuse, Sexual Abuse: A Journal of Research and Treatment, 14: 3148.

Langevin R (1983) Sexual strands: Understanding and treating sexual anomalies in men, Hillsdale, NJ: Erlbaum. 
Langevin, R., Curnoe, S. and Bain, J. (2000) A study of clerics who commit sexual offences: Are they different from other sex offenders? Child Abuse \& Neglect, 24: 535-545.

Langevin R., Wright, P. and Handy, L. (1989) Characteristics of sex offenders who were sexually victimised as children, Annals of Sex Research, 2: 227-253.

Lanyon, R.I. (1991) Theories of Sexual Offending, in C.R. Hollin and K. Howells, (Eds.) Clinical approaches to sex offenders and their victims, (pp.35-54), Oxford: Wiley.

Latzman, N.E., Viljoen, J.L., Scalaro, M.J. and Ullman, D. (2011) Sexual Offending in Adolescence: A Comparison of Sibling Offenders and Nonsibling Offenders across Domains of Risk and Treatment Need, Journal of Child Sexual Abuse, 20: 245-263.

Lawson, C. (1993) Mother-son sexual abuse: Rare or underreported? A Critique of the research, Child Abuse \& Neglect, 17: 261-269.

Lawson, L. and Chaffin, M. (1992) False negatives in sexual abuse disclosure interviews. Incidence and influence of caretaker's belief in abuse in cases of accidental abuse discovery by diagnosis of STD, Journal of Interpersonal Violence, 7: 532-542.

Leach, C., Stewart, A. and Smallbone, S. (2016) Testing the sexually abused-sexual abuser hypothesis: A prospective longitudinal birth cohort study, Child Abuse \& Neglect, 51: 144153.

Leclerc, B., Proulx, J. and McKibben, A. (2005) 'Modus operandi of sexual offenders working or doing voluntary work with children and adolescents', Journal of Sexual Aggression, 11: 187-195.

Lee, J.K.P., Jackson, H.J., Pattison, P. and Ward, T. (2002) Developmental risk factors for sexual offending, Child Abuse \& Neglect, 26: 73-92.

Leonard, E. D. (1996) A Social Exchange Explanation for the Child Sexual Abuse Accommodation Syndrome, Journal of Interpersonal Violence, 11: 107-117. 
Lev-Wiesel, R. (2000) Quality of life in adult survivors of child sexual abuse who have undergone therapy, Journal of Child Sexual Abuse, 9: 1-13.

Levenson, J.S., and MacGowan, M.J. (2004) Engagement, Denial, and Treatment Progress among Sex Offenders in Group Therapy, Sexual Abuse: A Journal of Research and Treatment, 16: 49-63.

Lewis, C.F. and Stanley, C.R. (2000) Women accused of sexual offences, Behavioural Sciences and the Law, 18: 73-81.

Longdon, C. (1993) A survivor and therapist's viewpoint, in M. Elliott (1993) (Ed.) Female Sexual Abuse of Children: The Ultimate Taboo, (pp.47-56), UK: Longman.

Lovell, E. (2002) Children and young people who display sexually harmful behaviour, Available at: http://www.nspcc.org.uk/Inform/research/Briefings/sexually harmful behaviuor_wda48213

(Accessed 25.4.08).

Lovett, B.B. (2004) Child sexual abuse disclosure: maternal response and other variables impacting the victim, Child and Adolescent Social Work, 21: 355-371.

Maikovich-Fong, A. and Jaffee, S. (2010) Sex differences in childhood sexual abuse characteristics and victims' emotional and behavioural problems: Findings from a national sample of youth, Child Abuse \& Neglect, 34: 429-437.

Maniglio, R. (2011) The role of childhood trauma, psychological problems, and coping in the development of deviant sexual fantasies in sexual offenders, Clinical Psychology Review, 31: 748-756.

Margolin, L. (1994) Child Sexual Abuse by Uncles: A Risk Assessment, Child Abuse \& Neglect, 18: 215-224.

Margolin, L. (1992) Sexual Abuse by Grandparents, Child Abuse \& Neglect, 16: 735-741. 
Margolin, L. and Craft, J.L. (1990) Child Abuse by Adolescent Caregivers, Child Abuse \& Neglect, 14: 365-373.

Marshall, W.L. (1999) Current status of North American assessment and treatment programs for sexual offenders, Journal of Interpersonal Violence, 14: 221-239.

Marshall, W.L. (1989) Invited essay: Intimacy, loneliness and sexual offenders, Behaviour Research and Therapy, 27: 491-503.

Marshall, W.L. and Barbaree, H.E. (1990) An integrated theory of the etiology of sexual offending, in W.L.Marshall, D.R. Laws and H.E. Barbaree, Handbook of sexual assault: Issues, theories, and treatment of the offender, (pp.257-275), New York: Plenum Press.

Marshall, W.L, Serran, G.A. and Cortoni, F.A. (2000) Childhood attachments, sexual abuse and their relationship to adult coping in child molesters, Sexual Abuse: A Journal of Research and Treatment, 12: 17-26.

Martin, J. (2016) Child Sexual Abuse Images Online: Implications for Social Work Training and Practice, British Journal of Social Work, 46: 372-388.

Martin, G., Bergen, H.A., Richardson, A.S., Roeger, L. and Allison, S. (2004) 'Sexual abuse and suicidality: gender differences in a large community sample of adolescents', Child Abuse \& Neglect, 28: 491-503.

Masson, H., Hackett, S., Phillips, J. and Balfe, M. (2015) Developmental markers of risk or vulnerability? Young females who sexually abuse - characteristics, backgrounds, behaviours and outcomes, Child and Family Social Work, 20: 19-29.

Mathews, R., Hunter, J.A. and Vuz, J. (1997) 'Juvenile female sex offenders: Clinical characteristics and treatment issues', Sexual Abuse: A Journal of Research and Treatment, 9: 187-199.

Mathews, R., Mathews, J.K. and Speltz, K. (1989) Female sexual offenders: An exploratory study, Orwell, VT: Safer Society Press. 
Mathis, J. L. (1972) Clear thinking about sexual deviations: A new look at an old problem, Chicago: Nelson-Holt.

McAlinden, A.M. (2014) Deconstructing Victim and Offender Identities in Discourses on Child Sexual Abuse, British Journal of Criminology, 54: 180-198.

McAlinden, A.M. (2006) 'Setting 'Em Up: Personal, Familial and Institutional Grooming in the Sexual Abuse of Children', Social \& Legal Studies, 15: 339-362.

McCartan, F.M., Law, H., Murphy, M. and Bailey, S. (2011) Child and adolescent females who present with sexually abusive behaviours: A 10-year UK prevalence study, Journal of Sexual Aggression, 17: 4-14.

McGrath, S.A., Nilsen, A.A. and Kerley, K.R. (2011) Sexual victimization in childhood and the propensity for juvenile delinquency and adult criminal behaviour: A systematic review, Aggression and Violent Behavior, 16: 485-492.

McGregor, K., Julich, S., Glover, M. and Gautam, J. (2010) Health Professionals’ Responses to Disclosure of Child Sexual Abuse History: Female Child Sexual Abuse Survivors' Experiences, Journal of Child Sexual Abuse, 19: 239-254.

McGuffey, C. (2008) "Saving masculinity”: Gender reaffirmation, sexuality, race, and parental responses to male child sexual abuse, Social Problems, 55:216-237.

McGuire, M. (1995) What works: Reducing reoffending, Chichester: Wiley.

McGuire, M and Dowling, S. (2013) Cyber-enabled crimes, Home Office Research Report 75, Available at:

https://www.gov.uk/government/uploads/system/uploads/attachment_data/file/246749/horr75 -summary.pdf (Accessed 2.7.15).

McLeod, D.A. (2015) Female offenders in child sexual abuse cases: A national picture, Journal of Child Sexual Abuse, 24: 97-114. 
McPhail, B.A. (2016) Feminist Framework Plus: Knitting Feminist Theories of Rape Etiology Into a Comprehensive Model, Trauma, Violence \& Abuse, 17: 314-329.

Meadows, P., Tunstill, J., George, A., Dhudwar, A. and Kurtz, Z. (2011) The costs and consequences of child maltreatment, Available at:

http://www.nspcc.org.uk/globalassets/documents/research-reports/costs-and-consequenceschild-maltreatment.pdf (Accessed 2.7.15)

Medlicott, D. (2004) Condemned to artifice and prevented from being a pirate: How prisoners convicted of terrible crimes recognize themselves in discourse, in R. Hamilton and M. Breen (Eds.), This thing of darkness: Perspectives on evil and human wickedness (pp.7892), Amsterdam, the Netherlands: Rodopi.

Mellor, D. and Deering, R. (2010) Professional responses and attitudes toward femaleperpetrated child sexual abuse: A study of psychologists, psychiatrists, probationary psychologists and child protection workers, Psychology, Crime \& Law, 16: 415-438.

Mendel, M.P. (1995) The Male Survivor: The Impact of Sexual Abuse, London: Sage.

Messman-Moore, T. and Brown, A. (2004) Child maltreatment and perceived family environment as risk factors for adult rape: Is child sexual abuse the most salient experience? Child Abuse \& Neglect, 28: 1019-1034.

Miller, L. (2013) Sexual offenses against children: Patterns and motives, Aggression and Violent Behavior, 18: 506-519.

Miller, D. and Brown, J. (2014) 'We have the right to be safe': Protecting disabled children from abuse, London: NSPCC, Available at: http://www.nspcc.org.uk/globalassets/documents/research-reports (Accessed 21.10.14).

Ministry Of Justice (2016) Offender Management Statistics Quarterly: July to September 2015, Available at: https://www.gov.uk/government/statistics/offender-managementstatistics-quarterly-july-to-september-2015 (Accessed 4.4.16) 
Ministry Of Justice (2016) Prison population 31 ${ }^{\text {st }}$ December 2015, Available at: https://www.gov.uk/government/statistics/offender-management-statistics-quarterly-july-toseptember-2015 (Accessed 4.4.16)

Ministry of Justice (2015) Multi-Agency Public Protection

Arrangements Annual Report 2014/15, Available at:

https://www.gov.uk/government/uploads/system/uploads/attachment_data/file/471408/mappa -annual-report-2014-15.pdf (Accessed 6.4.16).

Ministry of Justice (2015) Criminal Justice Statistics Quarterly Update to September 2014, Available at:

https://www.gov.uk/government/uploads/system/uploads/attachment_data/file/405301/cjsquarterly-update-september-2014.pdf (Accessed 19.6.15).

Ministry of Justice (2014) Criminal Justice Statistics 2014, Available at: https://www.gov.uk/government/uploads/system/uploads/attachment_data/file/428932/crimin al-justice-statistics-december-2014.pdf (Accessed 19.6.15).

Moulden, H., Firestone, P. and Wexler, A. (2007) Child Care Providers who Commit Sexual Offences: A Description of Offender, Offence, and Victim Characteristics, International Journal of Offender Therapy and Comparative Criminology, 51: 384-406.

Mullen, P.E., Martin, J.L., Anderson, J.C., Romans, S.E. and Herbison, G.P. (1996), Child Abuse \& Neglect, 20: 7-21.

Muskens, M., Bogaerts, S. van Casteren, M. and Labrijn, S. (2011) Adult female sexual offending: A comparison between co-offenders and solo offenders in a Dutch sample, Journal of Sexual Aggression, 17: 46-60.

Nalavany, B., Ryan, S. and Hinterlong, J. (2009) Externalizing behaviour among adopted boys with preadoptive histories of child sexual abuse, Journal of Child Sexual Abuse, 18: 553-573.

Nathan, P. and Ward, T. (2001) Females who Sexually Abuse Children: Assessment and 
Treatment Issues, Psychiatry, Psychology and Law 8: 44-55.

Noll, J.G. (2008) Sexual abuse of children - Unique in its effects on development? Child Abuse \& Neglect, 32: 603-605.

NOTA (n/d) Young People with Sexually Harmful Behaviour (SHB): Information Note 7, Available at:

http://www.nota.co.uk/Content/Media/08d84efefd6245658e177fb753806f6e/5Youngpeoplew ithSHB.pdf (Accessed 20.7.16).

NSPCC (2016) 5 child sex offences reported every hour, Available at: https://www.nspcc.org.uk/fighting-for-childhood/news-opinion/child-sex-offences-uk-recordrise/ (Accessed 30.3.16).

NSPCC (2015) Child protection register statistics in the UK, Available at: http://www.nspcc.org.uk/preventing-abuse/child-abuse-and-neglect/child-sexual-abuse (Accessed 15.6.15)

NSPCC (2013a) Giving Victims A Voice: A Joint MPS and NSPCC report into allegations of sexual abuse made against Jimmy Savile under Operation Yewtree, Available at: https://www.nspcc.org.uk/globalassets/documents/research-reports/yewtree-report-givingvictims-voice-jimmy-savile.pdf (Accessed 20.7.16).

NSPCC (2013b) Child sexual abuse: An NSPCC Research Briefing, London: NSPCC.

NSPCC (2013c) Harmful Sexual Behaviour: Research Briefing, London: NSPCC.

NSPCC (2009) Childline Casenotes: calls to Childline about sexual abuse, London: NSPCC.

NSPCC (2007) Childline Casenotes: calls to Childline about sexual abuse, London: NSPCC.

NSPCC (various dates) FOI requests sent to all police forces in England and Wales. 
Nunes, K.L, Hermann, C.A., Malcolm, J.R. and Lavoie, K. (2013) Childhood sexual victimization, pedophilic interest, and sexual recidivism, Child Abuse \& Neglect, 37: 703711.

Oaksford, K. and Frude, N. (2003) The Process of Coping Following Child Sexual Abuse: A Qualitative Study, Journal of Child Sexual Abuse, 12: 41-72.

Oaksford, K.L. and Frude, N. (2001) 'The Prevalence and Nature of Child Sexual Abuse: Evidence from a Female University Sample in the UK’, Child Abuse Review, 10: 49-59.

Oakley, A. (1981) Interviewing Women: A Contradiction in Terms, In H. Roberts (Ed.) Doing Feminist Research, London: Routledge and Kegan Paul.

O’Ciardha, C. and Ward, T. (2013) Theories of Cognitive Distortions in Sexual Offending: What the Current Research Tells Us, Trauma, Violence \& Abuse, 14: 5-21.

O’Ciardha, C. and Gannon, T.A. (2012) The implicit theories of firesetters: A preliminary conceptualization, Aggression and Violent Behavior, 17: 122-128.

O’Ciardha, C. and Gannon, T.A. (2011) The cognitive distortions of child molesters are in need of treatment, Journal of Sexual Aggression, 17: 130-141.

Office for National Statistics (2016) Abuse during childhood: Findings from the Crime Survey for England and Wales, year ending March 2016, Available at:

https://www.ons.gov.uk/peoplepopulationandcommunity/crimeandjustice/articles/abusedurin gchildhood/findingsfromtheyearendingmarch2016crimesurveyforenglandandwales (Accessed 12.8.16).

Ogloff, J.R.P., Cutajar, M.C., Mann, E. and Mullen, P. (2012) Child sexual abuse and subsequent offending and victimisation: A 45 year follow-up study, Trends \& Issues in Crime \& Criminal Justice No. 440, Canberra: Australian Institute of Criminology.

O’Leary, P.J. (2009) Men who were sexually abused in childhood: coping strategies and comparisons in psychological functioning, Child Abuse \& Neglect, 33: 471-479. 
O’Leary, P., Coohey, C. and Easton, S.D. (2010) The effect of severe child sexual abuse and disclosure on mental health during adulthood, Journal of Child Sexual Abuse, 19: 275-289.

O’Leary, P.J. and Gould, N. (2009) Exploring Coping Factors amongst Men Who Were Sexually Abused in Childhood, British Journal of Social Work, 40: 2669-2686.

O’Leary, P.J. and Barber, J. (2008) Gender differences in silencing following childhood sexual abuse, Journal of Child Sexual Abuse, 17: 133-143.

Oliver, B. (2007) Preventing Female-Perpetrated Sexual Abuse, Trauma, Violence and Abuse, 8: 19-32.

Paine, M.L. and Hansen, D.J. (2002) Factors influencing children to self-disclose sexual abuse, Clinical Psychology Review, 22: 271-295.

Parkinson, P.N., Oates, R.K. and Jayakody, A.A. (2012) Child sexual abuse in the Anglican Church, Journal of Child Sexual Abuse, 21: 553-570.

Pereda, N., Guilera, G., Forns, M. and Gomez-Benito, J. (2009) The international epidemiology of child sexual abuse: A continuation of Finkelhor (1994), Child Abuse \& Neglect, 33: 331-342.

Peter, T. (2008) Speaking About the Unspeakable: Exploring the Impact of Mother-Daughter Sexual Abuse, Violence Against Women, 14: 1033-1053.

Peter, T. (2006) Mad, Bad, or Victim? Making Sense of Mother-Daughter Sexual Abuse Feminist Criminology, 1: 283-302.

Petrillo, M. (2007) Power struggle: Gender issues for female probation officers in the supervision of high risk offenders, Probation Journal, 54: 394-406

Petry, S. S. D. (2005). The impact on male therapists treating sex offenders: a phenomenological study with a focus on gender, race and ethnicity, Doctoral dissertation, USA: Seton Hall University. 
Phelan, P. (1995) Incest and its meaning: The perspectives of fathers and daughters, Child Abuse \& Neglect, 19: 7-24.

Pierce, R. and Pierce, L.H. (1985) The Sexually Abused Child: A Comparison of Male and Female Victims, Child Abuse \& Neglect, 9: 191-199.

Plummer, K. (1995) Telling sexual stories: Power, change and social worlds, London: Routledge.

Plummer, C.A. (2006) The discovery process: What mothers see and do in gaining awareness of the sexual abuse of their children, Child Abuse \& Neglect, 30: 1227-1237.

Plummer, M. and Cossins, A. (2016) The Cycle of Abuse: When Victims Become Offenders, Trauma, Violence \& Abuse, doi: 10.1177/1524838016659487.

Priebe, G. and Svedin, C.G. (2008) Child sexual abuse is largely hidden from the adult society. An epidemiological study of adolescents' disclosures, Child Abuse \& Neglect, 32: 1095-1108.

Proeve, M. and Reilly, E. (2007) Personal and Offending Characteristics of Child Sexual Offenders Who Have Been Sexually Abused, Psychiatry, Psychology and Law, 14: 251-259.

Punch, K. F. (2005) Introduction to Social Research, London, Sage.

Quayle, E. and Taylor, M. (2002) Paedophiles, Pornography and the Internet: Assessment Issues, British Journal of Social Work, 32: 863-875.

Rabalais, A.E. (2003) The Cycle of Abuse: Factors that put survivors at risk for perpetration of child physical and/or sexual abuse, PhD thesis: West Virginia University, US, Available at:.

https://www.researchgate.net/profile/Joseph_Scotti/publication/266405947_The_Cycle_of_A buse_Factors that_Put_Survivors_at_Risk_for_Perpetration_of_Child_Physical_andor_Sexu al_Abuse/links/5702c30a08aea09bb1a303a2.pdf, (Accessed 6.7.15). 
Radford, L., Corral, S., Bradley C., Fisher, H., Bassett, C., Howatt, N. and Collishaw, S. (2011) Child abuse and neglect in the UK today, Available at: https://www.nspcc.org.uk/globalassets/documents/research-reports/child-abuse-neglect-uktoday-research-report.pdf (Accessed 25.6.15).

Reinhart, M.A. (1987) Sexually Abused Boys, Child Abuse \& Neglect, 11: 229-235.

Rich, P. (2011) Understanding, assessing and rehabilitating juvenile sexual offenders, New Jersey: Wiley.

Rind, B., Bauserman, R. and Tromovitch, P. (1998) A Meta-Analytic Examination of Assumed Properties of Child Sexual Abuse Using College Samples, Psychological Bulletin, 124: 22-53.

Robertiello, G. and Terry, K. J. (2007) Can we profile sex offenders? A review of sex offender typologies, Aggression and Violent Behaviour, 12: 508-518.

Roberts, S. (2011) Doing research with imprisoned adult male child sexual abusers: reflecting on the challenges, Child Abuse Review, 20: 187-196.

Roberts, S. and Vanstone, M. (2014) A Child Sexual Abuse Research Project: A Brief Endnote, Journal of Child Sexual Abuse, 23: 745-754.

Robson, C. (2002) Real World Research: A Resource for Social Scientists and PractitionerResearchers, Oxford: Blackwell Publishers.

Romano, E. and DeLuca, R. V. (2001) Male sexual abuse: A review of effects, abuse characteristics, and links with later psychological functioning, Aggression and Violent Behavior, 6: 55-78.

Romano, E. and DeLuca, R. V. (1997) Exploring the relationship between childhood sexual abuse and adult sexual perpetration, Journal of Family Violence, 12: 85-98. 
Romans, S.E., Gendall, K.A., Martin, J.L. and Mullen, P.E (2001) Child Sexual Abuse and Later Disordered Eating: A New Zealand Epidemiological Study, International Journal of Eating Disorders, 29: 380-392.

Russell, D.E.H. (1984a) Sexual exploitation, Beverley Hills, CA: Sage.

Russell, D.E.H. (1984b) The Prevalence and Seriousness of Incestuous Abuse: Stepfathers VS Biological Fathers, Child Abuse \& Neglect, 8: 15-22.

Russell, D.E.H. (1983) The Incidence and Prevalence of Intrafamilial and Extrafamilial Sexual Abuse of Female Children, Child Abuse \& Neglect, 7: 133-146.

Ryan, G. (2002) Victims who go on to victimize others: no simple explanations, Child Abuse \& Neglect, 26: 891-892.

Safeguarding Vulnerable Groups Act (2006) London: Stationery office.

Saied-Tessier, A. (2014) Estimating the costs of child sexual abuse in the UK, Available at: http://www.nspcc.org.uk/globalassets/documents/research-reports/estimating-costs-childsexual-abuse-uk.pdf, (Accessed 25.6. 15).

Salter, D., McMillan, D., Richards, M., Talbot, T., Hodges, J., Bentovim, A., Hastings, R., Stevenson, J. and Skuse, D. (2003) Development of sexually abusive behaviour in sexually victimised males: a longitudinal study, The Lancet, 361: 471-476.

Sandler, J. and Freeman, N.J. (2011) Female sex offenders and the criminal justice system: A comparison of arrests and outcomes, Journal of Sexual Aggression, 17: 61-76.

Saradjian, J. (1996) Women Who Sexually Abuse Children: From Research to Clinical Practice, Chichester: Wiley.

Save the Children (2011) Out from the shadows: sexual violence against children with disabilities, Available at: https://www.savethechildren.org.uk/sites/default/files/docs/out_of_the_shadows_5.pdf 
Schonbucher, V., Maier, T., Mohler-Kuo, M., Schnyder, U. and Landolt, M.A. (2012) Disclosure of Child Sexual Abuse by Adolescents: A Qualitative In-Depth Study, Journal of Interpersonal Violence, 27: 3486-3513.

Seghorn, T.K., Prentky, R.A. and Boucher, R.J.M.S. (1987) Childhood Sexual Abuse in the Lives of Sexually Aggressive Offenders, Journal of the American Academy of Child \& Adolescent Psychiatry, 26: 262-267.

Seto, M.C. and Eke, A.W. (2005) The future offending of child pornography offenders, Sexual Abuse, 17: 201-210.

Sex Offenders Act (1997), London: Stationery Office.

Sexual Offences Act (2003) London: Stationery Office.

Sgroi, S.M. (1982) Handbook of clinical intervention in child sexual abuse, Lexington, MA: Lexington Books.

Sgroi, S. M. and Sargent, N. M. (1993) Impact and treatment issues for victims of childhood sexual abuse by female perpetrators in M. Elliott (Ed.) Female Sexual Abuse of Children: The Ultimate Taboo (pp.5-14), Longman: Harlow.

Shelby, R.A., Stoddart, R.M. and Taylor, K.L. (2001) Factors contributing to levels of burnout among sex offender treatment providers, Journal of Interpersonal Violence, 16: 1205-1217.

Simmel, C., Postmus, J.L. and Lee, I. (2012) Sexual Revictimization in Adult Women: Examining Factors Associated with Their Childhood and Adulthood Experiences, Journal of Child Sexual Abuse, 21: 593-611.

Simons, D.A., Wurtele, S.K. and Durham, R.L. (2008) 'Developmental experiences of child sexual abusers and rapists', Child Abuse \& Neglect, 32: 549-560. 
Simons, D., Wurtele, S.K. and Heil, P. (2002) Childhood Victimisation and Lack of Empathy as Predictors of Sexual Offending Against Women and Children, Journal of Interpersonal Violence, 17: 1291-1307.

Sinclair, B.B. and Gold, S.R. (1997) The psychological impact of withholding disclosure of child sexual abuse, Violence \& Victims, 12: 137-145.

Sirles, E.A. and Franke, P.J. (1989) Factors influencing mothers' reactions to intrafamily sexual abuse, Child Abuse \& Neglect, 13: 129-139.

Skuse, D., Bentovim, A., Hodges, J., Stevenson, J., Andreou, C., and Lanyado, M. (1998) Risk factors for development of sexually abusive behaviour in sexually victimised adolescent boys: Cross sectional study, British Medical Journal, 317: 175-179.

Skuse, D., Stevenson, P. and Hodges, J. (1997) The influence of early experience of sexual abuse on the formulation of sexual preferences during adolescence, London, Institute of Child Health.

Smallbone, S. W. and Wortley, R.K. (2001) Child Sexual Abuse: Offender Characteristics and Modus Operandi, No. 193, Trends \& Issues in Crime and Criminal Justice, Australian Institute of Criminology: Canberra, Available at: http://www.aic.gov.au/media_library/publications (accessed 28.10.15).

Smart, C. (2000) Reconsidering the recent history of child sexual abuse, Journal of Social Policy, 29: 55-71.

Smith, C., Allardyce, S., Hackett, S., Bradbury-Jones, C., Lazenbatt, A. and Taylor, J. (2014) Practice and policy in the UK with children and young people who display harmful sexual behaviours: an analysis and critical review, Journal of Sexual Aggression, 20: 267-280.

Smith, D.W., Letourneau, E.J., Saunders, B.E., Kilpatrick, D.G., Resnick, H.S. and Best, C.L. (2000) Delay in disclosure of childhood rape: Results from a national survey, Child Abuse \& Neglect, 24: 273-287. 
Smith, N., Dogaru, C. and Ellis, F. (2015) Hear Me. Believe Me. Respect Me. A survey of adult survivors of child sexual abuse and their experiences of support services, University Campus Suffolk and Survivors in Transition, Available at:

http://cdn.basw.co.uk/upload/basw_122305-1.pdf (Accessed 24.8.16).

Smolak, L. and Murnen, S.K. (2002) A Meta-Analytic Examination of the Relationship Between Child Sexual Abuse and Eating Disorders, International Journal of Eating Disorders, 31: 136-150.

Sorenson, T. \& Snow, B. (1991) How Children Tell: The Process of Disclosure in Child Sexual Abuse, Child Welfare League of America, Vol LXX,1: 3-14.

Sorsoli, L., Kia-Keating, M. and Grossman, F.K. (2008) “I Keep That Hush-Hush”: Male Survivors of Sexual Abuse and the Challenges of Disclosure, Journal of Counselling Psychology, 55: 333-345.

Spiegel, J. (2003) Sexual Abuse of Males: The SAM Model of Theory and Practice, New York: Brunner-Routledge.

Spiegel, J. (1995) The sexual abuse of males: A structural equation model of dynamics and effects, (Doctoral dissertation, Florida State University, August 1995), Dissertation Abstracts International, 58, 5708A.

Staller, K.M. and Nelson-Gardell, D. (2006) “A burden in your heart”: Lessons of disclosure from female preadolescent and adolescent survivors of sexual abuse, Child Abuse \& Neglect, 29: $1415-1432$.

Starzyk, K.B. and Marshall, W. (2003) Childhood family and personological risk factors for sexual offending, Aggression and Violent Behavior, 8: 93-105.

Stathopoulos, M. (2012) Sibling sexual abuse, Australian Centre for the Study of Sexual Assault, Available at:

http://www.aifs.gov.au/acssa/pubs/researchsummary (accessed 13.11.12) 
Steel, J., Sanna, L., Hammond, B., Whipple, J. and Cross, H. (2004) Psychological sequelae of childhood sexual abuse: abuse-related characteristics, coping strategies, and attributional style, Child Abuse \& Neglect, 28: 785-801.

Stein, M. (2006) Missing years of abuse in children’s homes, Child and Family Social Work, 11: $11-21$.

Stein, M. (2004) What Works for Young People Leaving Care? Barnardo’s: Barkingside.

Stoltenborgh, M., van Ijzendoorn, M.H., Euser, E.M. and Bakermans-Kranenburg, M.J. (2011) A Global Perspective on Child Sexual Abuse: Meta-Analysis of Prevalence Around the World, Child Maltreatment, 16: 79-101.

Strauss, A.L. (1987) Qualitative analysis for social scientists, Available at: https://books.google.co.uk/books?id=y16ww5ZsJ0AC\&printsec=frontcover\&dq=strauss +198 7\&hl=en\&sa=X\&ved=0ahUKEwj52P2V6K_PAhXjIMAKHSbKCjcQ6AEIJTAA\#v=onepag e\&q=strauss\%201987\&f=false UK: Cambridge University Press.

Strauss, A.L. and Corbin, J. (1990) Basics of Qualitative Research: Grounded Theory Procedures and Techniques, London: Sage Publications.

Strickland, S.M. (2008) Female Sex Offenders: Exploring Issues of Personality, Trauma, and Cognitive Distortions, Journal of Interpersonal Violence, 23: 474-489.

Stroud, D.D. (1999) Familial support as perceived by adult victims of childhood sexual abuse, Sexual Abuse: A Journal of Research and Treatment, 11: 159-175.

Sullivan, J. and Beech, A. (2002) Professional Perpetrators: Sex Abusers Who Use Their Employment to Target and Sexually Abuse the Children With Whom They Work, Child Abuse Review, 11: 153-167.

Sullivan, J. and Sheehan, V. (2016) What motivates sexual abusers of children? A qualitative examination of the Spiral of Sexual Abuse, Aggression and Violent Behavior, 30: 76-87. 
Sullivan P.M., and Knutson J.F. (2000) Maltreatment and disabilities: a population based epidemiological study. Child Abuse \& Neglect 24: 1257-1273.

Summit, R.C. (1983) The Child Sexual Abuse Accommodation Syndrome, Child Abuse \& Neglect, 7: 177-193.

Syed, F. and Williams, S. (1996) Case studies of female sexual offenders in the correctional service of Canada: Correctional Service of Canada.

Tang, C.S. (2002) Childhood experience of sexual abuse among Hong Kong Chinese college students, Child Abuse \& Neglect, 26: 23-37.

Testa, M. Hoffman, J.H. and Livingstone, J.A. (2011) Intergenerational transmission of sexual victimization vulnerability as mediated via parenting, Child Abuse \& Neglect, 35: 363371.

The Guardian. (2009a) Up to 64,000 women in UK are child sex offenders, Available at: https://www.theguardian.com/society/2009/oct/04/uk-female-child-sex-offenders (Accessed 15.10.10).

The Guardian. (2009b) Vanessa George jailed for child sex abuse, Available at: http://www.guardian.co.uk (Accessed 15.10.10).

The Telegraph (2001) Sarah's parents overwhelmed as jury hears of her 'violent death', Available at:

http://www.telegraph.co.uk/news/uknews/1363002/Sarahs-parents-overwhelmed-as-juryhears-of-her-violent-death.html, (Accessed 8.4.16).

Thomas, T.A. and Fremouw, W. (2009) Moderating variables of the sexual "victim to offender cycle” in males, Aggression and Violent Behavior, 14: 382-387.

Timmerman, M.C. and Schreuder, P.R. (2014) Sexual abuse of children and youth in residential care: An international review, Aggression and Violent Behavior, 19: 715-720. 
Tsopelas, C., Spyridoula, T., Petros, N. and Athanassios, D. (2012) Female perpetrators of sexual abuse of minors: What are the consequences for the victims? International Journal of Law and Psychiatry, 35: 305-310.

Tsopelas, C., Spyridoula, T. and Athanasios, D. (2011) Review on female sexual offenders: Findings about profile and personality, International Journal of Law and Psychiatry, 34: 122126.

Turton, J. (2013) Betrayal of Trust: Victims of Maternal Incest, in H. Warming (Ed.), Participation, Citizenship and Trust in Children's Lives (pp.73-92), Basingstoke: Palgrave MacMillan.

Turton, J. (2010) Female sexual abusers: Assessing the risk, International Journal of Law, Crime and Justice, 38: 279-293.

Turton, J. (2008) Child Abuse, Gender and Society, Routledge: Abingdon.

Tutty, L.M., McConnell, S., Barter, K. and Fairholm, J. (2009) What Canadian youth tell us about disclosing abuse, Child Abuse \& Neglect, 33: 699-708.

Ullman, S.E. (2005) Ethnicity and child sexual abuse experiences of female college students, Journal of Child Sexual Abuse, 14: 67-89.

UNICEF (2014) Hidden in Plain Sight: A statistical analysis of violence against children, Available at:

http://files.unicef.org/publications/files/Hidden_in_plain_sight_statistical_analysis_EN_3_Se pt_2014.pdf (Accessed 22.8.16).

United States General Accounting Office (1996) Cycle of Sexual Abuse, Washington: US General Accounting Office.

Utting, W. (1997) People Like Us: The Report of the Review of Safeguards for Children Living away from Home. London: The Stationery Office. 
Vandiver, D.M. (2006) Female sex offenders: a comparison of solo offenders and cooffenders, Violence and Victims, 21: 339-354.

Vandiver, D.M. and Kercher, G. (2004) Offender and Victim Characteristics of Registered Female Sexual Offenders in Texas: A Proposed Typology of Female Sexual Offenders, Sexual Abuse: A Journal of Research and Treatment, 16: 121-137.

Vandiver, D.M. and Walker, J.T. (2002) Female sex offenders: An overview and analysis of 40 cases, Criminal Justice Review, 27: 284-300.

Vanstone, M. (2007) Supervising Offenders in the Community: a History of Probation Theory and Practice, Aldershot: Ashgate.

Veneziano, C., and Veneziano, L. (2002) Adolescent Sex Offenders: A Review of the Literature, Trauma, Violence \& Abuse, 3: 247-260.

Veneziano, C., Veneziano, L. and LeGrand, S. (2000) The Relationship Between Adolescent Sex Offender Behaviors and Victim Characteristics with Prior Victimization, Journal of Interpersonal Violence, 15: 363-374.

Vizard, E., Hickey, N., French, L. and McCrory, E. (2007) Children and adolescents who present with sexually abusive behaviour: A UK descriptive study, The Journal of Forensic Psychiatry \& Psychology, 18: 59-73.

Waldram, J.B. (2007) Everybody Has a Story: Listening to Imprisoned Sexual Offenders, Qualitative Health Research, 17: 963-970.

Walklate, S. (2001) Gender, crime and criminal justice, Devon, UK: Willan.

Wallis, K.M. (1995) Perspectives on child molesters, in Briggs F. (1995) From Victim to Offender: How Child Sexual Abuse Victims Become Offenders, (pp.1-17), NSW Australia: Allen \& Unwin. 
Ward, T. (2014) The explanation of sexual offending: from single factor theories to integrative pluralism, Journal of Sexual Aggression, 20: 130-141.

Ward, T. (2006) Strength-Based Treatment Theories in T. Ward, L.L. Polaschek, and A.R. Beech (2006), (Eds.) Theories of Sexual Offending, (pp.297-313), Chichester: Wiley.

Ward, T. and Beech, A. (2006) An integrated theory of sexual offending, Aggression and Violent Behavior, 11: 44-63.

Ward, T. and Keenan, T. (1999) Child Molesters' Implicit Theories, Journal of Interpersonal Violence, 14: 821-838.

Ward, T., Polaschek, L.L. and Beech, A.R. (2006), (Eds.) Theories of Sexual Offending, Chichester: Wiley.

Watkins, B. and Bentovim, A. (1992) The sexual abuse of male children and adolescents: A review of current research, Journal of Child Psychology and Psychiatry, 33: 197-248.

Weatherred, J.L. (2015) Child Sexual Abuse and the Media: A Literature Review, Journal of Child Sexual Abuse, 24: 16-34.

Weeks, R. and Widom, C. (1998) Self-reports of early childhood victimization among incarcerated adult male felons, Journal of Interpersonal Violence, 13: 346-361.

Welfare, A. (2008) How qualitative research can inform clinical interventions in families recovering from sibling abuse, Australia and New Zealand Journal of Family Therapy, 29: 139-147.

Wellard, S. (2001) Cause and Effect, Community Care 1364: 26-27.

Whitaker, D J, Le, B, Hanson R K, Baker, C K, McMahon PM, Ryan G, Klein A. and Rice, D. (2008) Risk factors for the perpetration of child sexual abuse: A review and meta-analysis, Child Abuse \& Neglect, 32: 529-548. 
Widom, C.S. (1995) Victims of Childhood Sexual Abuse - Later Criminal Consequences, National Institute of Justice, Research Brief, Available at:

https://www.ncjrs.gov/pdffiles/abuse.pdf (accessed 15.7.15)

Widom, C.S. (1989) The cycle of violence, Science, 244: 160-166, Available at: http://www.columbia.edu/cu/psychology/courses/3615/Readings/Science_1989_Widom_160 6.pdf (accessed 16.7.15).

Widom, C.S., Czaja, S.J. and Dutton, M.A. (2008) Childhood victimization and lifetime revictimization, Child Abuse \& Neglect, 32: 785-796.

Widom, C.S. and Ames, M.A. (1994) Criminal Consequences of Childhood Sexual Victimization, Child Abuse \& Neglect, 18: 303-318.

Widom, C.S. and Morris, S. (1997) Accuracy of adult recollections of childhood revictimisation: Part 2, Childhood sexual abuse, Psychological Assessment, 9: 34-46.

Wijkman, M., Bijleveld, C. and Hendriks, J. (2011) Female sex offenders: Specialists, generalists and once-only offenders, Journal of Sexual Aggression, 17: 34-45.

Wilcox, D.T. Richards, F. and O’Keeffe, Z.C. (2004) 'Resilience and risk factors associated with experiencing childhood sexual abuse', Child Abuse Review, 13: 338-352.

Williams, M.C. (1995) Partnership between Parents, Children and Local Authorities in Relation to the Children Act 1989, Unpublished PhD thesis: University of Wales Swansea.

Wolfe, D.A., Sas, L. and Wekerle, C. (1994) Factors associated with the development of posttraumatic stress disorder among child victims of sexual abuse, Child Abuse \& Neglect, 18: $37-50$.

World Health Organisation (2006) Preventing child maltreatment: a guide to taking and generating evidence, Geneva: World Health Organisation. 
Worling, J. (2012) The assessment and treatment of deviant sexual arousal with adolescents who have offended sexually, Journal of Sexual Aggression, 18: 36-63.

Worling, J. R. (1995) Sexual Abuse Histories of Adolescent Male Sex Offenders: Differences on the Basis of the Age and Gender of Their Victims, Journal of Abnormal Psychology, 10: 610-613.

Wyre, R. (2000) Paedophile characteristics and patterns of behaviour: Developing and using a typology, in C. Itzin (2000) (Ed.) Home Truths About Child Sexual Abuse, (pp.49-69), Abingdon: Routledge.

Yates, P., Allardyce, S. and MacQueen, S. (2012) Children who display harmful sexual behaviour: Assessing the risks of boys abusing at home, in the community or across both settings, Journal of Sexual Aggression, 18: 23-35. 